\title{
INTERDIGITAL CAPACITIVE MICROMACHINED ULTRASONIC TRANSDUCERS FOR MICROFLUIDIC APPLICATIONS
}

\author{
A Dissertation Presented to \\ The Academic Faculty \\ By \\ Jeffrey J. McLean
}

In Partial Fulfillment of the Requirements for the Degree

Doctor of Philosophy in Mechanical Engineering

Georgia Institute of Technology

August 17, 2004

Copyright 2004 by Jeffrey J. McLean 


\section{INTERDIGITAL CAPACITIVE MICROMACHINED ULTRASONIC TRANSDUCERS FOR MICROFLUIDIC APPLICATIONS}

Approved by:

Dr. F. Levent Degertekin, Chair

Dr. Yves Berthelot

Dr. Peter Hesketh
Dr. A. Bruno Frazier

Dr. Mark Prausnitz

Dr. Wenjing Ye

August 17, 2004 


\section{ACKNOWLEDGEMENTS}

First and foremost, I would like to thank my wife, Drue, for her support and encouragement during my time at Georgia Tech. We have enjoyed living in Atlanta and now look forward to a new chapter of our life. I also thank my entire family, especially

my parents, for their support. I thank Levent Degertekin for all of his help during the course of my research in the role of both advisor and friend. All the members of Levent's research group have been great to work with and I regard all of them as friends. I especially thank Neal Hall, Josh Knight, Matt Abercrombie, and Guclu Onaran for their help in the cleanroom, the lab and in life. All the members of the MEMS research group in the ME department have been very helpful and for that I am grateful. The staff of the MiRC cleanroom has been a pleasure to work with, and I thank them for keeping the cleanroom in excellent condition. I would also like to thank the members of my thesis committee - Levent Degertekin, Yves Berthelot, Peter Hesketh, Bruno Frazier, Mark Prausnitz, and Wenjing Ye - for reviewing my dissertation and for their insights. Finally, I thank the DARPA Microsystems Technology Office and the Research Partnership to Secure Energy for America for their financial support. 
List of Figures $\quad$ vi

List of Tables $\quad$ xiv

$\begin{array}{ll}\text { Summary } & \text { Xv }\end{array}$

Chapter 1 Background and Motivation 1

Micropumps $\quad 2$

Large-Scale Ultrasonic Sensors 4

Micromachined Ultrasonic Transducers 5

Chapter 2 Interdigital cMUT Operation 13

Chapter 3 Analytical Model for Acoustic Mode Propagation 22

$\begin{array}{ll}\text { Free Plate } & 23\end{array}$

Fluid Loaded Plate $\quad 33$

Fluid Filled Microchannel $\quad 38$

Chapter 4 Coupled-Field Finite Element Model of cMUT Arrays 46

Development of Finite Element Model $\quad 47$

Analysis of Baffled Piston $\quad 47$

Infinitely Long Free Plate $\quad 51$

Fluid Loaded Plate $\quad 55$

Simulation of Interdigital cMUT Array in Fluid Half-space $\quad 59$

$\begin{array}{ll}\text { Model Validation } & 60\end{array}$

$\begin{array}{ll}\text { Parametric Studies } & 63\end{array}$

Interdigital cMUT Array in Fluid-Filled Microchannel 69

Guided Mode Generation $\quad 70$

$\begin{array}{ll}\text { Parametric Studies } & 72\end{array}$

Chapter 5 Low-Temperature cMUT Fabrication 75

Review of Established cMUT Fabrication Processes $\quad 78$

$\begin{array}{ll}\text { CMUT Fabrication Process } & 80\end{array}$

Bottom Electrode Formation $\quad 82$

Sacrificial Layer Formation $\quad 82$

Membrane Depostion $\quad 84$

Top Electrode Formation $\quad 85$

Second Membrane Deposition $\quad 86$

Membrane Release $\quad 86$

Membrane Sealing $\quad 90$

Membrane Thinning 91 
Bond Pad Etch $\quad 92$

Via Drilling 93

Plasma Processing $\quad 94$

Silicon Nitride Film Characterization $\quad 95$

$\begin{array}{ll}\text { Discussion } & 96\end{array}$

Chapter 6 Electrical Characterization $\quad 97$

Electrostatic Actuation of cMUT Membranes $\quad 97$

$\begin{array}{ll}\text { Equivalent Circuit Model } & 101\end{array}$

$\begin{array}{ll}\text { Impedance Measurements } & 105\end{array}$

Chapter 7 Fluid Sensing $\quad 119$

Directionality Measurements $\quad 122$

$\begin{array}{ll}\text { Guided Mode Excitation } & 126\end{array}$

Zero-Crossing Measurements 132

Sensitivity to Changes in Fluid Sound Speed 138

$\begin{array}{ll}\text { Sensitivity to Changes in Temperature } & 142\end{array}$

Sensitivity to Changes in Flowrate 146

$\begin{array}{ll}\text { Multi-Mode Ultrasonic Fluid Sensor } & 148\end{array}$

$\begin{array}{ll}\text { Multi-Mode Ultrasonic Fluid Sensor } & 148\end{array}$

Discussion 151

Chapter 8 Fluid Pumping $\quad 154$

Acoustic Streaming $\quad 155$

Governing Equations 155

Acoustic Streaming Field in a Rectangular Microchannel 157

Efficiency of Acoustic Streaming 164

$\begin{array}{ll}\text { Characterization of Acoustic Streaming Pump } & 166\end{array}$

Acoustic Heating 173

Discussion

Chapter 9 Conclusions $\quad 182$

Recommendations 185

$\begin{array}{ll}\text { Appendix } 1 \text { Code for Analytical Model } & 187\end{array}$

Appendix 2 Finite Element Model for cMUT Array 191

$\begin{array}{lr}\text { Appendix } 3 \text { cMUT Fabrication Process } & 198\end{array}$

$\begin{array}{ll}\text { References } & 202\end{array}$ 


\section{LIST OF FIGURES}

Figure 1 Illustration of typical membrane pump.

Figure 2 The motion of ions in an electro-osmotic pump causes the fluid to flow from anode to cathode.

Figure 3 Illustration of FPW device used for acoustic pumping and sensing.

Figure 4 Time-of-flight system used for measuring flow rate in pipes. A sound pulse travels from Piezo 1 to Piezo 2 first in the direction of fluid flow and then against.

Figure 5 Illustration of Doppler sensing technique used in pipes. 5

Figure 6 Schematic view of a single element of a cMUT. 6

Figure 7 Mode shape of the Scholte wave propagating along the interface of a water half space and a silicon plate $200 \mu \mathrm{m}$ thick.

Figure 8 Illustration of structure Wang et al. used to generate guided acoustic waves. 11

Figure 9 Image showing a small section of a cMUT array element designed for ultrasonic imaging. Image courtesy of the Khuri-Yakub Group at Stanford University. 14

Figure 10 Schematic of a typical interdigital acoustic transducer.

Figure 11 Illustration of QUIDT consisting of a thin-film piezoelectric on a thin silicon nitride membrane.

Figure 12 Schematic cross-section view (a) and micrograph showing completed Scholte wave transducer designed for operation at $10 \mathrm{MHz}(\mathrm{b})$. Note the sine and cosine inputs to the transducer.

Figure 13 Pulse echo measurement.

Figure 14 Guided mode transmit receive measurement. 20

Figure 15 Illustration of interdigital cMUT used for immersion measurements. 21

Figure 16 Illustration of coordinate system and boundary conditions for a free plate. 25

Figure 17 Plot of the determinant of the coefficient matrix calculated as a function of the phase velocity. 28

Figure 18 Geometric dispersion of the phase velocity of propagating modes for a free plate. 
Figure 19 Profile of x-direction displacement over the thickness of a $200 \mu \mathrm{m}$ plate at $10 \mathrm{MHz}(\mathrm{f}-\mathrm{d}=2 \mathrm{MHzmm})$. Profile is for the $\mathrm{S}_{0}$ mode with a phase velocity of $8018 \mathrm{~m} / \mathrm{s}$.

Figure 20 Profile of $x$-direction displacement over the thickness of a $200 \mu \mathrm{m}$ plate at $10 \mathrm{MHz}(\mathrm{f}-\mathrm{d}=2 \mathrm{MHzmm})$. Profile is for the $\mathrm{A}_{0}$ mode with a phase velocity of $3866 \mathrm{~m} / \mathrm{s}$.

Figure 21 Illustration of coordinate system and boundary conditions for a fluid-loaded plate.

Figure 22 Geometric dispersion of the Scholte wave for a fluid half-space attached to a silicon plate.

Figure 23 Displacement field for the Scholte wave at the interface of a water half-space and a $200 \mu \mathrm{m}$ plate at $10 \mathrm{MHz}$.

Figure 24 Illustration of coordinate system and boundary conditions for a fluid-filled microchannel.

Figure 25 Dispersion of phase velocity for propagating modes as a function of the channel height in a water filled channel at $10 \mathrm{MHz}$. The top and bottom substrates are made of $200 \mu \mathrm{m}$ thick silicon plates.

Figure 26 Profile of displacement in the x-direction for the Scholte wave in a $100 \mu \mathrm{m}$ channel at $10 \mathrm{MHz}$. Note that the displacement in the fluid is more than 50 times greater than the displacement in the plates.

Figure 27 Profile of displacement in the x-direction for the first guided mode in a $100 \mu \mathrm{m}$ channel at $10 \mathrm{MHz}$.

Figure 28 Dispersion of group velocity for modes propagating in a water filled channel of variable height at $10 \mathrm{MHz}$. The plates forming the channel are $200 \mu \mathrm{m}$ thick silicon.

Figure 29 Illustration of the model of a vibrating piston on a baffle.

Figure 30 Plot of the real and imaginary parts of the radiation impedance of a baffled piston

Figure 31 Illustration of the arrangement of attenuating regions of the silicon plate. 52

Figure 32 Illustration of method used to excite Lamb waves in the silicon plate. $\quad 52$

Figure 33 Plot of the amplitude of the normal surface displacement over the length of the silicon plate. The damping coefficient for this case was $5 \times 10^{-10}$. 
Figure 34 Plot of magnitude of normal surface displacement for optimal lossy silicon region.

Figure 35 Illustration of arc of fluid coupled to the vibrating silicon plate.

Figure 36 Plot of the pressure distribution in the fluid resulting from the propagation of $\mathrm{A}_{0}$ Lamb waves in the silicon plate.

Figure 37 Fourier transform of normal surface displacement of silicon plate attached to water half-space and excited with point forces at $10 \mathrm{MHz}$.

Figure 38 Fourier transform of normal surface displacement of a silicon plate with a single vibrating membrane attached to water half-space. 58

Figure 39 Illustration of finite element model for fluid half-space.

Figure 40 Finite element analysis result for pressure fields in water where the fingers of the interdigital cMUT shown in Fig. 1 are driven at $10 \mathrm{MHz}$ with signals: (a) in phase, (b) $-90^{\circ}$ out of phase, and (c) $+90^{\circ}$ out of phase. The total lateral distance in the shown area is $7 \mathrm{~mm}$.

Figure 41 Fourier transform of normal surface displacement corresponding to forward and backward excitation of the Scholte wave.

Figure 42 Directionality of an interdigital cMUT array as a function of the number of finger pairs in a fluid half space.

Figure 43 Efficiency in generating Scholte waves as a function of the number of fingers.

Figure 44 Efficiency as a function of the normalized wavelength for a 10 finger transducer.

Figure 45 Directionality as a function of phase for a 10-finger interdigital cMUT.

Figure 46 FEM results for an interdigital cMUT in a water half-space illustrating the total radiated power, total power in the Scholte wave, and directional Scholte wave power as a function of phase.

Figure 47 Illustration of finite element model for fluid filled microchannel.

Figure 48 Pressure distribution in (a) $30 \mu \mathrm{m}$ and (b) $90 \mu \mathrm{m}$ channels resulting from forward excitation of 5 finger pair Scholte wave transducer at $10 \mathrm{MHz}$.

Figure 49 FT of normal surface displacement plotted against the phase velocity of the propagating modes for channel heights of 30,90 , and $120 \mu \mathrm{m}$. 
Figure 50 FEM results for an interdigital cMUT in a $100 \mu \mathrm{m}$, water-filled channel illustrating the total radiated power, total power in the guided waves, and the directional guided wave power as a function of phase.

Figure 51 (a) Scaled drawing of sensing devices designed for operation at $10 \mathrm{MHz}$. The spacing between the transmitter and receiver is $8.26 \mathrm{~mm}$. (b) Close-up image of a single array which has overall dimensions of $0.64 \mathrm{~mm}$ by $0.300 \mathrm{~mm}$.

Figure 52 Scaled drawing of pumping device designed for operation at $10 \mathrm{MHz}$. This transducer has 20 finger pairs to generate highly directional Scholte waves. 77

Figure 53 Schematic of completed immersion cMUT fabricated with the low temperature, CMOS compatible process.

Figure 54 Illustration of low-temperature process flow for immersion cMUTs. The major steps are: (1) Bottom electrode formation, (2) Sacrificial layer formation, (3) Membrane deposition, (4)Top electrode formation, (5)Second membrane deposition, (6) Membrane release, (7) Membrane sealing, and (8) Via drilling 81

Figure 55 Mask image (a) and picture of chromium sacrificial layer (b). Each membrane is $100 \mu \mathrm{m}$ by $20 \mu \mathrm{m}$.

Figure 56 Mask image of patterned top electrode.

Figure 57 Mask image (a) and picture of etch holes used for releasing the membrane (b).

Figure 58 Illustration of non-uniform membrane gap (a) resulting from poor selectivity of the release agent. The gap thickness remains constant (b) when the chromium sacrificial layer is used due to the high selectivity of the chromium etch. $\quad 88$

Figure 59 Illustration of the etch progression during membrane release.

Figure 60 SEM showing the local sealing of the cMUT membrane around the etch hole resulting from a PECVD silicon nitride deposition.

Figure 61 (a) Mask image and (b) scanning electron micrograph (SEM) of completed cMUT showing the protected sealing locations.

Figure 62 Mask image showing the location of the through-wafer vias.

Figure 63 Illustration of first-order model of a cMUT.

Figure 64 Schematic of cMUT membrane. 
Figure 66 Standard representation of the mechanical impedance of a cMUT membrane.

Figure 67 Schematic of setup used for standard impedance measurements.

Figure 68 Complex impedance of a cMUT in air at bias voltages of 0,80 , and 100V. 107

Figure 69 Mode shape of $100 \mu \mathrm{m}$ long, $20 \mu \mathrm{m}$ wide, $0.8 \mu \mathrm{m}$ thick silicon nitride membrane resulting from a modal analysis in ANSYS.

Figure 70 Complex impedance of a cMUT in water at bias voltages of 0,80 , and $100 \mathrm{~V}$.

Figure 71 Complex impedance of tuned cMUT in water half-space with 100V DC bias.

Figure 72 Equivalent circuit used for calculating the transmission coefficient.

Figure 73 Real impedance of interdigital cMUT placed in water-filled microchannel at different levels of DC bias.

Figure 74Change in real power consumed by an interdigital cMUT in a water-filled, $100 \mu \mathrm{m}$ deep microchannel.

Figure 75 System used to measure the impedance of a single leg of an interdigital cMUT with a phased neighbor.

Figure 76 Schematic of phasing applied to legs of interdigital transducer for measurement of (a) Leg 1 and (b) Leg 2.

Figure 77 Change in power consumed by a single leg of an interdigital cMUT with a vibrating neighbor.

Figure 78 FEM results for power radiated by the individual legs of an interdigital cMUT as a function of phase.

Figure 79 Illustration of pulse-echo measurement used for sensing changes in fluid properties in a microchannel.

Figure 80 Typical pulse-echo response from an ultrasonic transducer in a microchannel.

Figure 81 Schematic of experimental setup used for sensing experiments in fluid halfspace.

Figure 82 Signals recorded by the cMUT receiver when the transmitter is operated to achieve forward (bottom waveform) and backward (top waveform) excitation at 
$10 \mathrm{MHz}$ in a water half-space. The transmitter is excited with a 10 cycle tone burst.

Figure 83 Schematic of experimental setup used for directionality experiments in fluid channel.

Figure 84 Signals recorded by the cMUT receiver when the transmitter is operated to achieve forward (bottom waveform) and backward (top waveform) excitation at $10 \mathrm{MHz}$ in a water filled channel $100 \mu \mathrm{m}$ deep. The transmitter is excited with a 10 cycle tone burst and is located $6.8 \mathrm{~mm}$ away from the receiver.

125

Figure 85 Schematic of experimental set-up used to perform transmit-receive measurements in a fluid-filled microchannel.

Figure 86 Illustration of guided mode propagation resulting from impulse excitation of an interdigital cMUT in a $100 \mu \mathrm{m}$ water filled microchannel.

Figure 87 Impulse response of transmit-receive interdigital cMUT in a100 $\mu$ m water-filled microchannel.

Figure 88 Dispersion curve illustrating the propagating modes in a $100 \mu \mathrm{m}$ water-filled channel.

Figure 89 Received signals for guided modes excited at 10, 12.7 and $17.4 \mathrm{MHz}$. Note that the signals for 12.7 and $17.4 \mathrm{MHz}$ have been scaled by factors of 3 and 25 , respectively.

Figure 90 Experimental data illustrating the change in phase velocity, and thus location of the zero crossing, resulting from a change in fluid properties. The gate signal tells the time interval counter which zero crossing to monitor.

Figure 91 (a) Illustration of system used to perform zero-crossing measurements and (b) photograph of actual experimental set-up.

Figure 92 Plot of the received signal amplitude as a function of DC bias.

Figure 93 Plot of the signal-to-noise ratio as a function of input $\mathrm{AC}$ voltage.

Figure 94 Jitter in the time interval measurement as a function of the SNR.

Figure 95 Change in phase of the received Scholte wave resulting from a change in the concentration of glycerol in de-ionized water.

Figure 96 Change in phase of the received guided wave resulting from a change in the concentration of glycerol in de-ionized water.

Figure 97 Sound speed in pure water as a function of temperature. 
Figure 98 Illustration of setup used for temperature measurement using the Scholte wave.

Figure 99 Scholte wave phase velocity as a function of temperature. The solid line represents the published values for the speed of sound in water as function of temperature. The error bars result from the jitter in the measurement.

Figure 100 Received waveforms for Scholte wave transmit-receive measurements in pure water with flow rates ranging from 0 to $4 \mathrm{~mL} / \mathrm{min}$ with $1 \mathrm{~mL} / \mathrm{min}$ increments. 147

Figure 101 Illustration of independent modes that can be excited in a microfluidic channel: 1) Bulk wave pulse-echo, 2) Scholte wave transmit-receive, 3) Guided mode transmit-receive.

Figure 102 Illustration of independent flow measurement.

Figure 103 Illustration of the analytical model for acoustic streaming.

Figure 104 Plot of the theoretical acoustic streaming field in a $100 \mu \mathrm{m}$ deep, water filled channel for a range of frequencies.

Figure 105 Plot of the acoustic streaming field in a $100 \mu \mathrm{m}$ deep, water filled channel for different plane wave amplitudes.

Figure 106 Plot of acoustic streaming field in a $100 \mu \mathrm{m}$ deep channel filled with pure water and pure glycerol.

Figure 107 Schematic (a) and photograph (b) of the experimental setup used for characterizing the acoustic pump.

Figure 108 Microfluidic channel in race-track configuration: (a) top view showing the flow path (b) cross section showing the fluid connections.

Figure 109 Illustration of power flow and various efficiencies in the acoustic streaming pump.

Figure 110 Volume flow rate as a function of power consumed by the cMUT.

Figure 111 Streaming efficiency as a function of power consumed by the cMUT. 172

Figure 112 Calibration system used for IR camera.

Figure 113 Temperature measured with calibrated thermocouple and IR camera. $\quad 175$

Figure 114 Generation of heat resulting form acoustic attenuation in the microchannel. (a) Picture of the device and (b) temperature distributions at various times showing acoustic heating. 
Figure 115 Average temperature of the fluid inside the channel as a function of time. 177 


\section{LIST OF TABLES}

Table 1 Sensitivity of first three guided modes in a $100 \mu \mathrm{m}$ channel at $10 \mathrm{MHz}$ to changes in material and geometric parameters.

Table 2 Periodicity of Scholte wave transducers designed for operation in water halfspaces and microchannels.

Table 3 Optimal values of parameters used to form the lossy silicon region. 54

Table 4 Recipes for PECVD of silicon nitride and amorphous silicon. 94

Table 5 Recipes for reactive ion etch of silicon nitride and amorphous silicon $\quad 94$

Table 6 Properties of silicon nitride film. 96

Table 7 Summary of phase velocity, group velocity and arrival time information for guided modes propagating in a $100 \mu \mathrm{m}$ water-filled channel.

Table 8 Properties of water-glycerol mixtures at $20^{\circ} \mathrm{C}$.

Table 9 Measurable quantities and dependent variables for various acoustic measurements

Table 10 Efficiency of $10 \mathrm{MHz}$ cMUT operation at its most efficient point. 


\section{SUMMARY}

The goal of this research was to develop acoustic sensors and actuators for microfluidic applications. To this end, capacitive micromachined ultrasonic transducers (cMUTs) were developed which generate guided acoustic waves in fluid half-spaces and microchannels. An interdigital transducer structure and a phased excitation scheme were used to selectively excite guided acoustic modes which propagate in a single lateral direction. Analytical models were developed to predict the geometric dispersion of the acoustic modes and to determine the sensitivity of the modes to changes in material and geometric parameters. Coupled field finite element models were also developed to predict the effect of membrane spacing and phasing on mode generation and directionality.

After designing the transducers, a surface micromachining process was developed which has a low processing temperature of $250^{\circ} \mathrm{C}$ and has the potential for monolithically integrating cMUTs with CMOS electronics. The fabrication process makes extensive use of PECVD silicon nitride depositions for membrane formation and sealing. The fabricated interdigital cMUTs were placed in microfluidic channels and demonstrated to sense changes in fluid sound speed and flow rate using Scholte waves and other guided acoustic modes. The minimum detectable change in sound speed was $0.25 \mathrm{~m} / \mathrm{s}$, and the minimum detectable change in flow rate was $1 \mathrm{~mL} / \mathrm{min}$. The unique nature of the Scholte wave allowed for the measurement of fluid properties of a semi-infinite fluid using two transducers on a single substrate. Changes in water temperature, and thus sound speed, were measured and the minimum detectable change in temperature was found to be $0.1^{\circ} \mathrm{C}$. For fluid pumping, interdigital cMUTs were integrated into microchannels and excited with phase-shifted, continuous wave signals. Highly directional guided waves 
were generated which in turn generated acoustic streaming forces in the fluid. The acoustic streaming forces caused the fluid to be pumped in a single, electronicallycontrolled direction. For a power consumption of $43 \mathrm{~mW}$, a flow rate of $410 \mathrm{~nL} / \mathrm{min}$ was generated against a pressure of $3.4 \mathrm{~Pa}$; the thermodynamic efficiency was approximately $5 \times 10^{-8} \%$. Although the efficiency and pressure head are low, these transducers can be useful for precisely manipulating small amounts of fluid around microfluidic networks. 


\section{CHAPTER 1}

\section{BACKGROUND AND MOTIVATION}

The goal of this research is to investigate the use of ultrasound for microfluidic applications and develop novel sensor and actuator technology based on micromachined transducers. Ultrasonic transducers are particularly attractive for micro-scale applications since there are no moving parts and surface micromachining allows integration with other types of sensors. For fluid sensing applications, ultrasound is attractive since the temperature, viscosity and sound speed of the liquid can be determined without disturbing the flow like cantilever [1] or thermal anemometer [2] based sensors. Ultrasound can also be used for fluid pumping and mixing due to nonlinear acoustic effects such as acoustic streaming and radiation pressure [3].

Microfluidic devices use manufacturing technologies developed for integrated circuits to build systems which manipulate small amounts of liquid. These micro electromechanical systems (MEMS) manipulate fluid samples ranging in volume from $100 \mathrm{nl}$ to $10 \mu \mathrm{l}$ [4]. Some of the applications for such devices include bio-MEMS for performing biological analyses [5] and lab-on-a-chip systems for chemical analyses [6]. Reducing the size of these systems to the micro-scale has several advantages [4]. First, the volume of reagents and waste is drastically reduced. Second, the small size allows many tests to be performed in parallel for improved accuracy and reliability. And finally, there is the potential for low cost devices owing to the well established integrated circuit manufacturing processes. To fully realize the aforementioned benefits of these microfluidic devices, it would be advantageous if most of the required functions were 
integrated onto a single chip. These functions include pumping fluids around the device, mixing reagents, and sensing changes in flow and fluid properties. In the following sections, existing types of micropumps and ultrasonic fluid sensors and sensing techniques are described.

\section{Micropumps}

Micropumps are used to move fluid through the channels of a microfluidic device and can be classified into two main categories: membrane pumps and field-induced pumps. Membrane pumps are essentially positive displacement pumps that require bulk motion of a diaphragm. These pumps are capable of providing large pressure heads and flow rates, but this is not always desirable for biological applications where accurately manipulating small fluid samples is the goal. Membrane pumps can be actuated using piezoelectric transducers [7], shape memory alloys [8], or electrostatic forces [9]. These membrane pumps are typically fabricated by stacking several wafers together, as shown in Figure 1, which limits the potential for chip-scale integration.

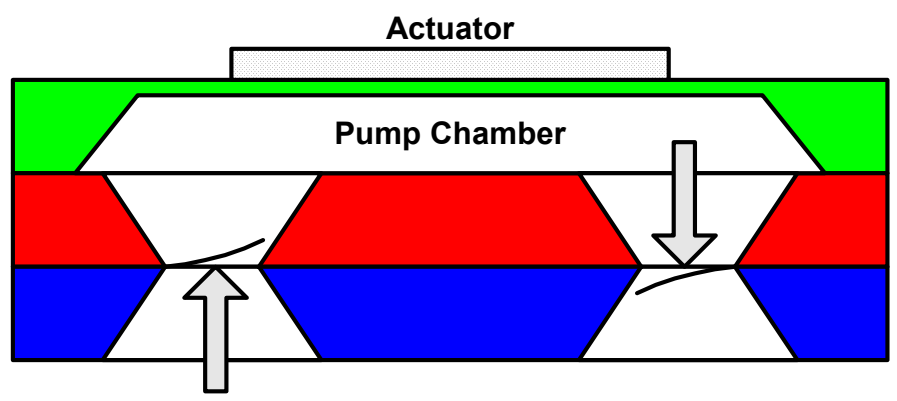

Figure 1 Illustration of typical membrane pump. 
The flow direction is controlled through the use of valves which can be either active or passive. However, when particles such as cells are introduced into the flow, the valves can become clogged which negatively impacts the performance of the pump.

Field-induced pumps are generally simpler to integrate than membrane pumps, but the existing technologies still have limitations. The most common field-induced pump uses the electro-osmotic effect to pump liquids in microchannels. In an electro-osmotic pump, an electric field is applied across a conductive fluid [10]. The motion of the ions from anode to cathode causes the bulk fluid to move in the direction of the electric field, as shown in Figure 2.

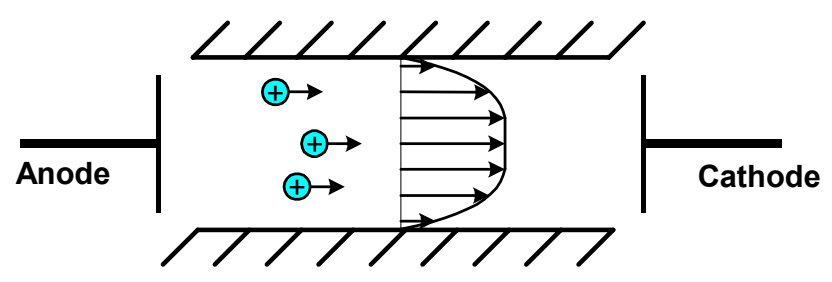

Figure 2 The motion of ions in an electro-osmotic pump causes the fluid to flow from anode to cathode.

Although this can provide a simple, integrated solution to fluid pumping, there are some limitations. First, the required voltages for some electro-osmotic pumps are quite high, on the order of thousands of volts [11]. Second, electro-osmosis does not provide a generic pumping solution since the effect is highly dependent on the electrical and viscous properties of the fluid.

Another type of field-induced pump, the flexural plate wave (FPW) pump, uses acoustic streaming forces to pump liquids [12]. For these transducers, a flexural wave, such as a Lamb wave, is generated in a thin silicon nitride membrane using interdigital piezoelectric transducers, as shown in Figure 3. 


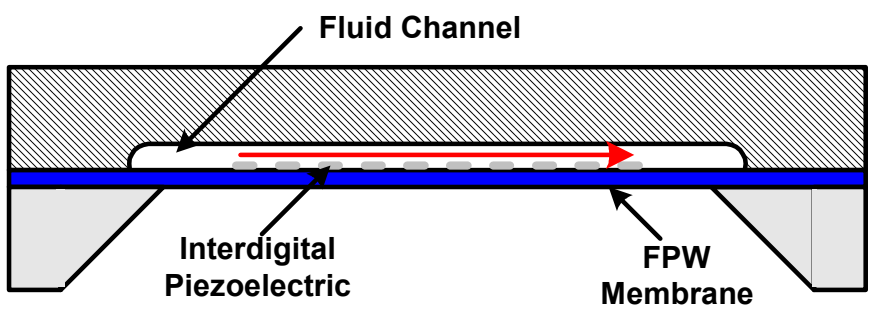

Figure 3 Illustration of FPW device used for acoustic pumping and sensing.

The flexural wave propagates at a velocity less than the sound speed in the fluid and thus generates an evanescent acoustic field in the liquid. When the amplitude of the acoustic wave is high, acoustic streaming forces generate a net flow in the liquid. This acoustic streaming technique is promising since it can pump nearly all fluids, but the thin, delicate membranes of FPW devices make integration very challenging.

\section{$\underline{\text { Large-Scale Ultrasonic Sensors }}$}

Ultrasound has been used extensively in large scale systems for monitoring flow and fluid properties, but has seen limited use in microfluidic environments. The key benefits of ultrasonic sensing are that there are no moving parts and the sensing does not affect the flow. For large scale systems, ultrasound is used for flow sensing using one of two methods. The first uses a pair of piezoelectric transducers on the outside of a pipe to send an acoustic pulse through the liquid in the pipe, as shown in Figure 4[13].

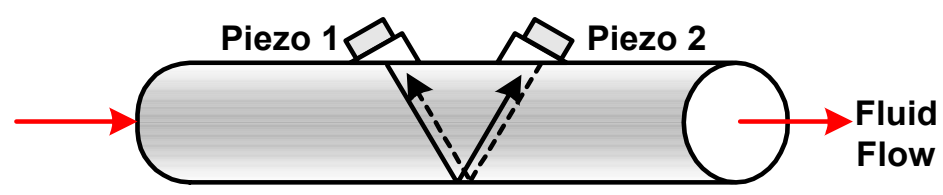

Figure 4 Time-of-flight system used for measuring flow rate in pipes. A sound pulse travels from Piezo 1 to Piezo 2 first in the direction of fluid flow and then against. 
By monitoring the change in the time of flight between a forward and backward propagating pulse, one can determine the average velocity of the liquid. The second method utilizes the Doppler effect by looking at the reflected acoustic waves coming off of particles or bubbles in the fluid, as shown in Figure 5[13].

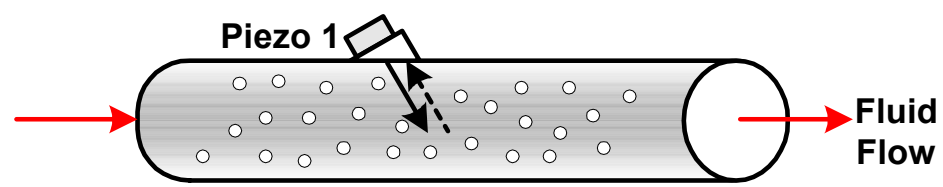

Figure 5 Illustration of Doppler sensing technique used in pipes.

The frequency shift of the reflected signal is a function of the flow velocity and provides the basis for the measurement.

Although ultrasonic sensing has some benefits, the sensing techniques and transducers cannot be simply scaled down for microfluidic applications. For the large scale ultrasonic sensing techniques, bulk piezoelectric transducers are used. The performance and body of knowledge surrounding bulk piezoelectrics makes them attractive, but there is a lower limit to the size of single crystal piezoelectrics due to the fabrication processes [14]. Additionally, integration of these transducers into microfluidic systems for mass production would be difficult.

\section{Micromachined Ultrasonic Transducers}

Thin film piezoelectrics were developed as an alternative to bulk piezoelectrics and allow smaller transducers to be fabricated. Thin film piezos are fabricated using surface micromachining processes and therefore ease integration with other microfluidic components. A few research groups have used thin film piezos such as zinc oxide for 
sensing and actuation in microchannels. Bradley et al. used interdigital zinc oxide transducers for exciting FPW devices for acoustic pumping and sensing [12]. Jagannathan et al. integrated zinc oxide transducers into microchannels and demonstrated the ability to mix fluids and sense changes in fluid temperature [15]. However, the piezoelectric properties of these thin films are inferior to those of the bulk material [14]. Additionally, acoustic matching layers are required to increase the efficiency of the transducers due to the impedance mismatch between the piezoelectric and the surrounding fluid [16].

Capacitive micromachined ultrasonic transducers (cMUTs) were developed in the mid 1990's as an alternative to piezoelectric transducers and have advantages in terms of bandwidth, dynamic range and insertion loss[17]. These transducers are surface micromachined using a sacrificial layer technique and can thus be fabricated into dense arrays. Small vibrating membranes are responsible for generating the ultrasonic waves and have negligible mechanical impedance as compared to fluids over a broad frequency range.

A single element of a cMUT consists of a compliant membrane with a moveable electrode separated from the bottom electrode by a vacuum sealed gap as shown in Figure 6. Many such membranes are grouped together in parallel to form a single transducer element.

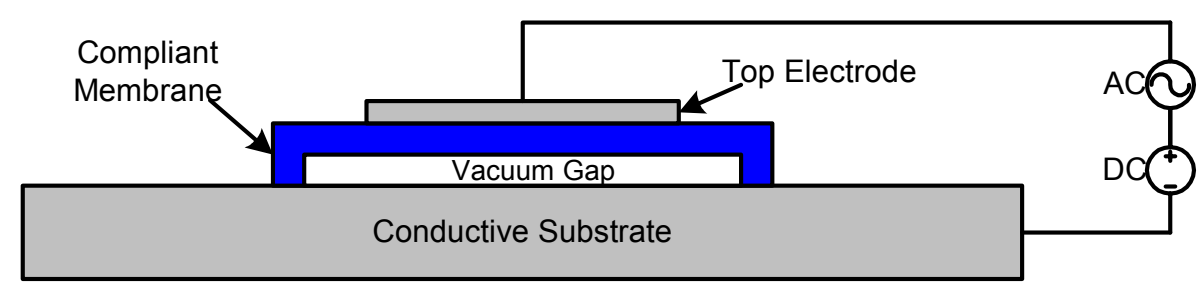

Figure 6 Schematic view of a single element of a cMUT. 
Application of an oscillating voltage in addition to a DC bias voltage generates electrostatic forces which set the membrane into motion at the input frequency. This motion is coupled to the surrounding fluid and the substrate, generating acoustic waves in both media. For reception, an incoming acoustic wave causes the membrane to deflect resulting in a measurable change in capacitance. The impedance of this vibrating membrane is much less than that of piezoelectrics which results in a decreased insertion loss and improved device performance over a large frequency band [16].

Some researchers have integrated cMUTs into microchannels and demonstrated the ability to mix fluids and sense changes in fluid temperature and or channel height [15]. However, most research has focused on the using cMUTs in 1D arrays for medical ultrasound imaging [17]. For these medical imaging applications, the aim is to selectively generate a bulk pressure wave in the fluid. Acoustic waves excited in the substrate and at the fluid-solid interface, Lamb waves and Scholte waves, respectively, are considered undesirable, spurious modes [17],[18]. In an attempt to use one of these spurious modes as a sensing tool, Yaralioglu et al. preferentially generated Lamb waves using interdigitated cMUTs on thin silicon membranes for airborne applications [19],[20]. Bradley et al. generated highly directional Lamb waves using interdigitated piezoelectric transducers for microfluidic applications [12]. Vellekoop et al. have used Lamb wave oscillators for liquid and gas sensors [21]. However, these flexural plate wave devices are fabricated on fragile, thin membranes making CMOS electronics integration and device customization difficult.

The other spurious mode generated by vibrating cMUT membranes, the Scholte wave, is particularly interesting for fluidic applications. This acoustic mode is similar to 
the better known Stoneley wave in that it propagates along the interface of two dissimilar materials [22]. The Stoneley wave is trapped at the interface two elastic solids and has a phase velocity that is less than the shear and longitudinal sound speeds in both media. It is therefore an evanescent wave whose amplitude decreases exponentially with distance away from the interface in both media. The Scholte wave, also referred to as the Stoneley-Scholte wave, can exist at the interface of a liquid and a solid [23]. This mode propagates along the liquid-solid interface with a phase velocity less than the sound speeds in both media. The resulting wave is therefore evanescent in both media and does not radiate energy into the fluid. The only diminution in wave amplitude comes from material attenuation. The evanescent decay of the wave amplitude is illustrated in Figure 7. In Figure 7, one can see that wave amplitude decays exponentially with distance away from the plate. Also the displacement in the x-direction (along the interface) in the fluid is more than 20 times larger than that in the solid, which is indicative of the fact that the majority of the energy in the wave is concentrated in the fluid near the surface. 


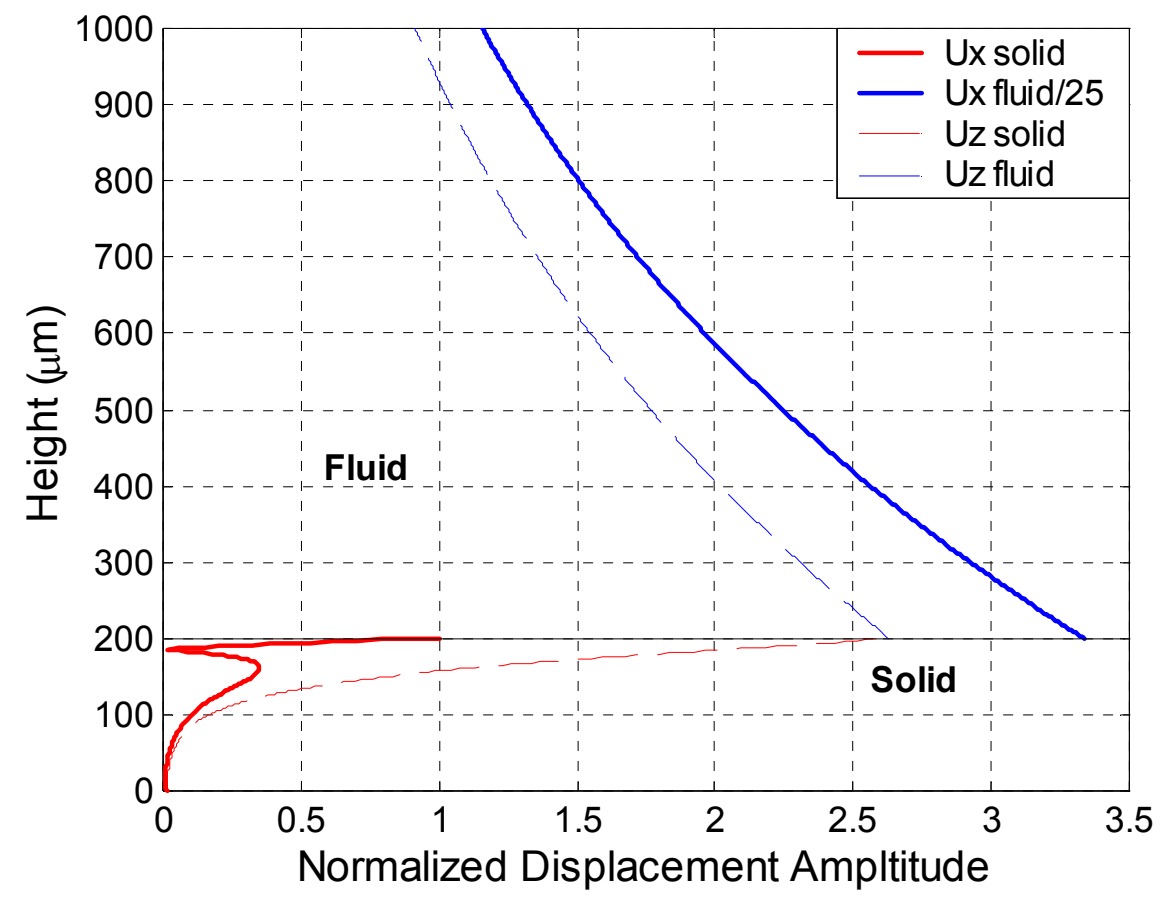

Figure 7 Mode shape of the Scholte wave propagating along the interface of a water half space and a silicon plate $200 \mu \mathrm{m}$ thick.

The energy concentration of the Scholte wave sparked the interest of other researchers, primarily for applications in non-destructive evaluation. Nasr et al. used interdigitated piezoelectric transducers to generate Scholte waves at a silica-water interface [24]. The interdigital piezoelectric transducers were fabricated by gluing sheets of polyvinyl diflouride (PVDF) piezoelectric onto lithographically defined chromium electrodes separated by a lateral distance of one wavelength. The transducers were demonstrated to generate bi-directional Scholte waves, but the fabrication process was costly and unreliable. Since a bi-directional Scholte wave is generated due to the one wavelength periodicity, at most only half of the power going into the Scholte wave reaches the receiver which limits the achievable signal-to-noise ratio for sensing applications. Desmet et al. excited Scholte waves at a mercury-plexiglass interface using 
a pulsed laser. The laser passed through an optically transparent layer, plexiglass in this case, and locally heated the absorbing medium, here mercury. This local heating generated thermo-elastic stresses which in turn generated acoustic waves such as bulk waves, leaky Rayleigh waves, and Scholte waves. The various waves were identified based on the time of arrival measured using an optical detection scheme [25]. Although this approach was successful in generating Scholte waves, the potential applications, especially for microfluidics, are limited because of challenges involved with integration. Tinel et al. developed a method for generating Scholte waves in various test materials using a removable transducer [26]. An interdigital piezoelectric transducer was fabricated on a glass plate to generate Schotle waves in the test specimen of interest. The glass substrate was held in intimate contact with the test specimen, an aluminum plate in this case, so that the acoustic mode generated by the transducer could be transferred to the plate. Tinel et al. verified the exponential decay and phase velocity of the Scholte wave for several materials. However, this technique for exciting Scholte waves does not lend itself well to micro-scale applications since it is difficult to align micromachined transducers with other substrates.

The generation and propagation of guided acoustic waves in layers of fluid separating two elastic solids is of particular interest for microfluidic applications and has been investigated theoretically and experimentally. Wang et al. first demonstrated the generation of guided acoustic waves in a layer of fluid separating two elastic plates [27], [28]. An interdigital electrode structure was fabricated on a piezoelectric substrate, as shown in Figure 8, and excited with a sinusoidal tone burst to generate a traveling Rayleigh wave. 


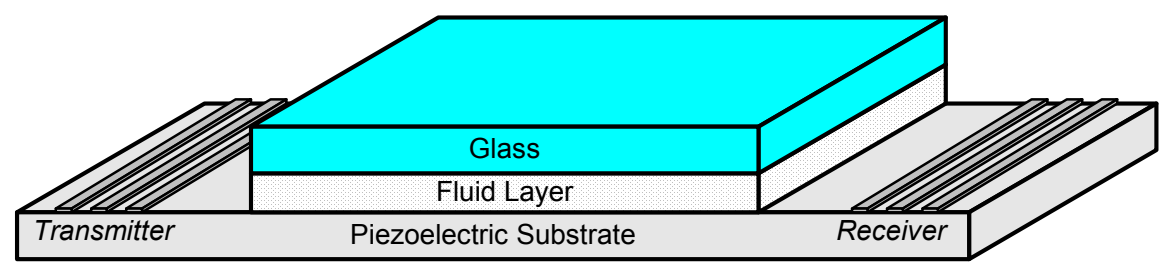

Figure 8 Illustration of structure Wang et al. used to generate guided acoustic waves.

The Rayleigh wave propagated along the surface of the substrate from the transmitter toward the receiver. When the surface wave reached the fluid, it was converted into a guided acoustic wave. Different modes were excited by changing the height of the fluid region. The researchers developed and experimentally verified a model for the propagating modes, but their transducer was not able to selectively excite different guided modes for a fixed height of fluid. Additionally, the models for predicting the phase and group velocities of the various modes were based on the assumption that the elastic plates were semi-infinite. This assumption is valid when the plate thickness is much larger than the wavelength of the acoustic modes in the solid but begins to fail when the plate thicknesses are decreased. Lec et al. used a very similar approach for generating guided acoustic waves in thin fluid layers but focused on the use of these waves for fluid sensing [29]. They demonstrated that the guided modes in the fluid were sensitive to changes in fluid properties and that some of the modes were very sensitive to changes in the thickness of the fluid layer. All of this work on guided acoustic waves made use of bulk piezoelectric substrates which severely limits the potential for integration into microfluidic systems.

In this research, interdigital cMUTs which generate highly directional Scholte waves and other guided modes in semi-infinite fluids and microchannels are used for 
sensing and actuation. The transducers are surface micromachined on a standard silicon substrate using a CMOS-compatible fabrication process to facilitate future electronics integration and to produce a more robust device. The fabrication process also provides the opportunity for integrating the transducers into microfluidic channels and combining them with other sensors. The interdigital configuration combined with a phased excitation scheme allows one to selectively generate Scholte waves and guided waves in a preferred lateral direction. The directional, single mode operation makes the transducers attractive on many fronts. From a fundamental physics perspective, the interdigital cMUTs are used to study the effect of changes in fluid properties and channel geometry on acoustic mode generation, mode propagation, and acoustic streaming. From an applications standpoint, Scholte wave transducers are demonstrated to sense changes in flow and fluid properties and to pump fluids in microchannels.

In the following chapters, the approach used for generating guided acoustic modes is presented before describing analytical and finite element models used for designing and analyzing the transducers. The low-temperature cMUT fabrication process is then presented before moving on to the experimental characterization of the transducers. 


\section{CHAPTER 2}

\section{INTERDIGITAL CMUT OPERATION}

In this research, capacitive micromachined ultrasonic transducers are arranged into an interdigital configuration to selectively excite guided acoustic modes in fluid halfspaces and fluid-filled microchannels. If a single membrane was immersed in fluid and excited with an impulse, the transducer would act as an acoustic point source. The resulting broadband pulse would excite all acoustic wave modes that could propagate in the system. However, little energy would be coupled to the fluid since the radiation impedance of a single vibrating membrane is mostly imaginary when the transducer is small compared to the acoustic wavelength [30]. To couple a larger amount of power to the fluid, the effective area of the transducer must be increased. Since the geometry of each membrane determines the frequency response of the transducer, one cannot simply make the membrane larger. To increase the effective area while maintaining the desired frequency response, many small membranes are packed closely together and connected in parallel, as shown in Figure 9. 


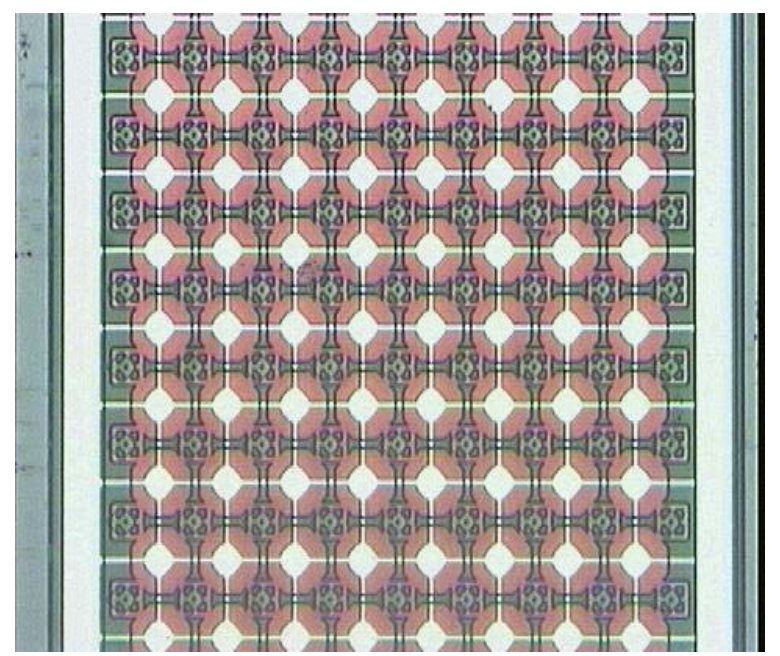

Figure 9 Image showing a small section of a cMUT array element designed for ultrasonic imaging. Image courtesy of the Khuri-Yakub Group at Stanford University.

The array shown in Figure 9 is composed of many circular membranes connected in parallel. The overall width of the transducer element is $350 \mu \mathrm{m}$.

If the goal is to excite a single acoustic mode, a different array structure must be used. Since the transducer is located on the surface of a plate, an acoustic mode can be selectively excited using an interdigital transducer (IDT) structure [31]. IDTs were originally developed for generating surface acoustic waves (SAW) on piezoelectric substrates for use as analog filters. Since their inception, countless sensors using surface acoustic waves have been developed including mass sensors, chemical sensors, and biosensors [32]. An interdigital transducer consists of parallel rows of long, thin electrodes, or fingers, arranged in an alternating sequence as illustrated in the following figure. 


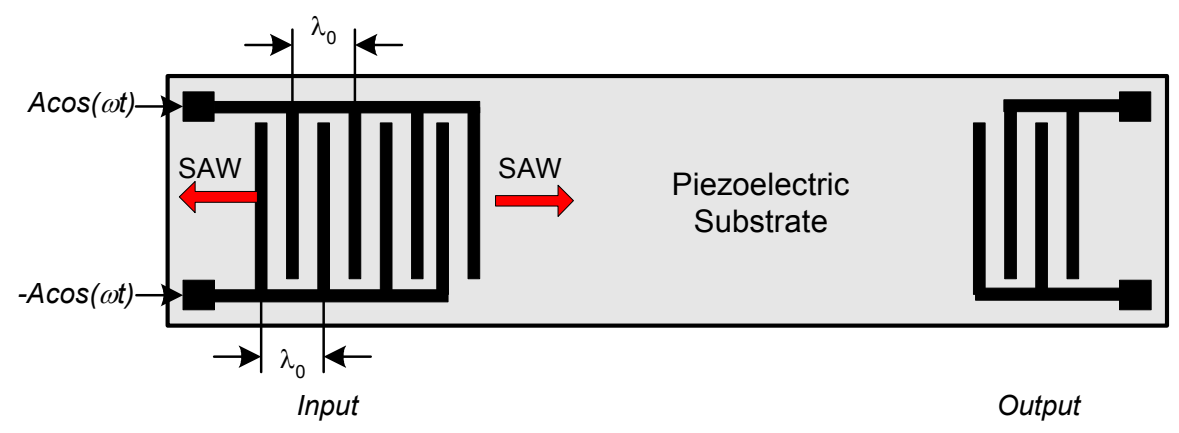

Figure 10 Schematic of a typical interdigital acoustic transducer.

The spacing between the neighboring electrodes in the IDT is typically one half of the wavelength of the desired acoustic mode, $\lambda_{0}$. The two legs of the transducer are excited with signals of equal amplitude and opposite polarity to generate a bi-directional surface acoustic wave in the piezoelectric substrate. As the number of electrode pairs, or fingers, is increased, the transducer begins to act more and more like a filter that selectively excites a single acoustic mode.

Placing transducers at one wavelength intervals selectively excites a particular guided acoustic wave, but this mode is inherently bi-directional: the same amount of energy propagates in both lateral directional. Several approaches have been used for producing directional surface acoustic waves [33], [34]. Most of these approaches make use of reflectors placed on the surface of the substrate at locations dictated by the wavelength of the acoustic mode. This type of approach cannot be used for generating directional Scholte waves or guided fluid waves since most of the energy is contained. This makes the modes insensitive to passive reflectors placed on the surface. Bustillo et al., therefore, developed an interdigital transducer which used a phased excitation scheme to generate directional Lamb waves in thin membranes [12]. These transducers, like other 
IDTs, consist of parallel rows of long rectangular electrodes. However, the spacing and phasing of these transducers are different. In this case, neighboring electrodes are placed at $\lambda / 4$ locations, where $\lambda$ is the wavelength of the mode of interest, and are driven $90^{\circ}$ out of phase. Many such groups, or fingers, are grouped together and placed at one wavelength intervals to form the transducer. This transducer structure has been referred to as a quadrature unidirectional interdigital transducer (QUIDT) and is shown schematically in Figure 11.

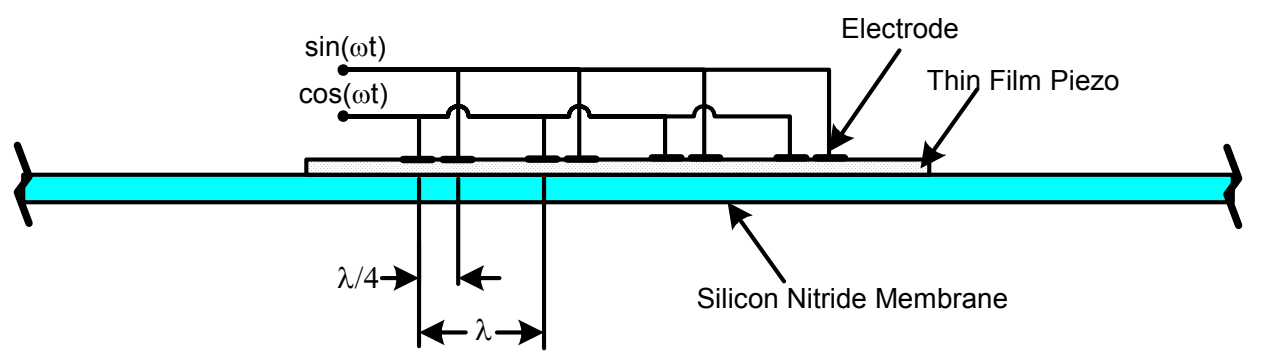

Figure 11 Illustration of QUIDT consisting of a thin-film piezoelectric on a thin silicon nitride membrane.

The ability of this transducer to generate a unidirectional wave can be understood by examining two time harmonic traveling waves, $p_{1}$ and $p_{2}$, that are shifted in space and time. The first wave represents the acoustic field generated by the transducer leg driven with the $\cos (\omega t)$ signal and can be written using complex exponentials as,

$$
p_{1}=A e^{j \omega t}\left[e^{-j k x}+e^{j k x}\right]
$$

where $A$ is the amplitude of the wave. The second wave is shifted in time by a phase of $\phi$ and in space by a distance of $\delta$ and can be written as

$$
p_{2}=B e^{j \omega t}\left[e^{-j k x} e^{-j \phi} e^{-j k \delta}+e^{j k x} e^{j \phi} e^{-j k \delta}\right]
$$


where $B$ is the amplitude of the wave. If the two amplitudes are taken to be equal, $A=B$, and the two pressure fields are added, one obtains

$$
p_{\text {Total }}=A e^{j \omega t}\left[e^{-j k x}\left(1+e^{-j(\phi+k \delta)}\right)+e^{j k x}\left(1+e^{j(\phi-k \delta)}\right)\right] .
$$

When $\phi=0$ and $\delta=0$, the amplitude of the pressure waves propagating in the $+x$ - and $-x$ directions is $2 A$ : the waves add constructively in both directions, as expected. When the phase is set to $90^{\circ}$, or $\pi / 2$, and the distance is set to $\lambda / 4$, one finds that the amplitude of the wave traveling in the $+x$-direction goes to zero, and the amplitude of the wave traveling in the $-x$-direction goes to $2 A$. The wave traveling in the $+x$-direction experiences destructive interference, while the wave traveling in the $-x$-direction experiences constructive interference. When the phase is set to $-\pi / 2$, the situation is reversed: the amplitude of the wave in the $+x$-direction becomes $2 A$ and the amplitude of the wave in the $-x$-direction goes to zero. Thus, if one can generate a single frequency sinusoidal single, place sources at $\lambda / 4$ locations, and drive neighboring sources with a phase difference of $90^{\circ}$, one can generate a unidirectional traveling wave. In practice, the wave will not be completely unidirectional since the length of the transducer is finite and thus harmonics are generated which do not cancel. However if the number of finger pairs is large, a nearly unidirectional traveling wave can be generated. This transducer design and excitation scheme is used in this research for generating directional guided acoustic modes using interdigital cMUTs and will now be described.

In this research, cMUTs are fabricated into an interdigital configuration to generate guided acoustic waves in liquids. The interdigital cMUTs consist of parallel rows of rectangular silicon nitride membranes separated by $\lambda / 4$, where $\lambda$ is the acoustic wavelength of the guided mode. Many fingers are grouped together and excited with 
phase shifted signals at the design frequency. With this structure, a traveling wave is generated which propagates in a single lateral direction, from transmitter toward receiver [12](Figure 12(a)). The micrograph of a typical interdigital cMUT operating at $10 \mathrm{MHz}$, where the Scholte wavelength is $146 \mu \mathrm{m}$, is shown in Figure 12(b). Each finger of this particular transducer consists of three membranes that are $20 \mu \mathrm{m}$ wide, $100 \mu \mathrm{m}$ long and $0.8 \mu \mathrm{m}$ thick. Since the cMUTs are surface micromachined using a PECVD-based process with a maximum processing temperature of $250^{\circ} \mathrm{C}$, they can be integrated into a variety of fluidic systems and integrated with electronics via post-CMOS processing [35]. This constitutes a major improvement over existing cMUT processes as well as over piezoelectric transducers. 


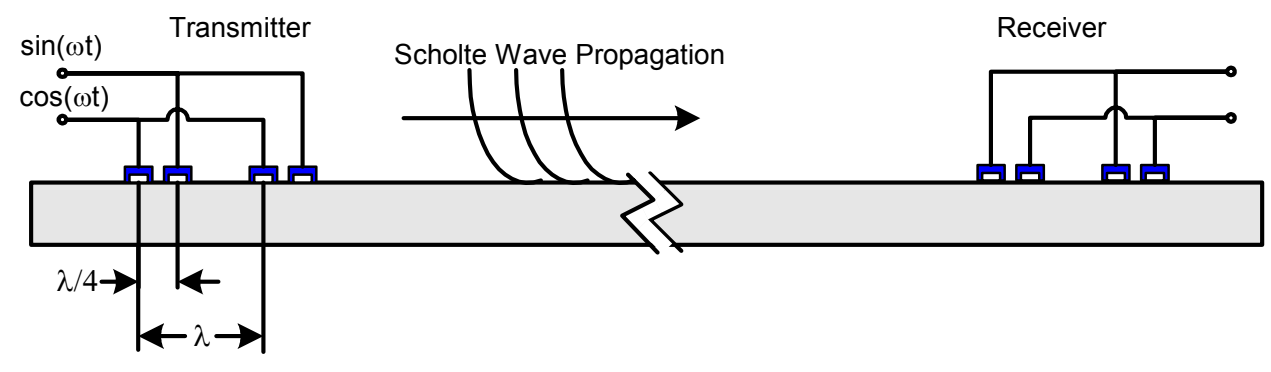

(a)

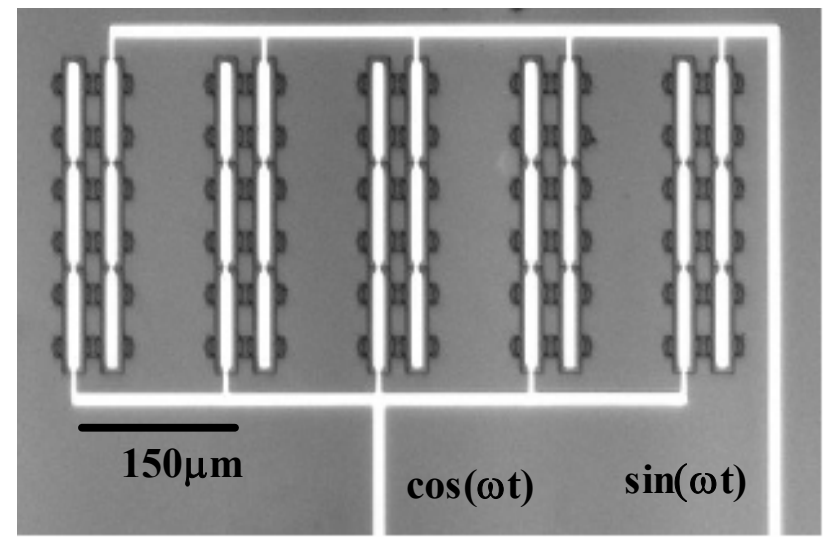

(b)

Figure 12 Schematic cross-section view (a) and micrograph showing completed Scholte wave transducer designed for operation at $10 \mathrm{MHz}(\mathrm{b})$. Note the sine and cosine inputs to the transducer.

The same interdigital cMUT can be used to perform two types of measurements: traditional bulk wave pulse-echo operation in a microchannel and guided wave transmitreceive operation in a fluid half-space or microchannel. For bulk wave pulse-echo measurements, both inputs of the cMUT are connected together and excited with a single pulse, as shown in Figure 13. 


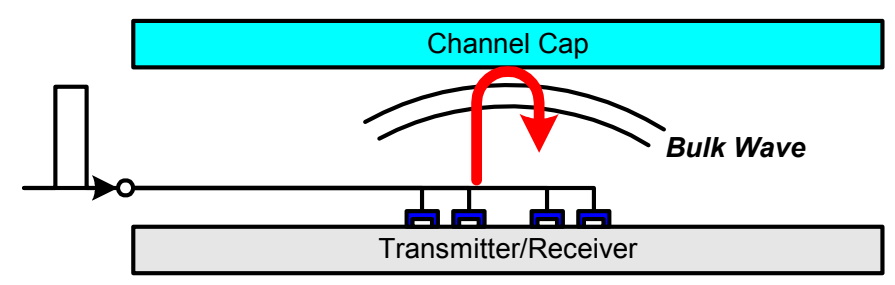

Figure 13 Pulse echo measurement.

By examining the time delay between the input and the first reflection from the channel ceiling, information on channel height and or fluid temperature can be obtained [15]. However, since both of these changes are manifested through a change in arrival time of the reflected pulse, it is difficult to obtain independent measurements.

The transmit-receive measurement is performed by exciting a guided wave which propagates predominantly in a single lateral direction, from transmitter toward receiver, as shown in Figure 14.

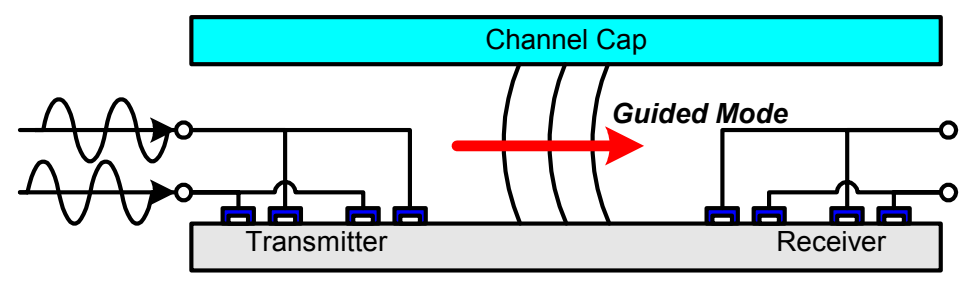

Figure 14 Guided mode transmit receive measurement.

The directional guided wave is generated by exciting the two inputs of the interdigital transducer independently with a phase shifted tone burst at the design frequency. Since the guided wave is sensitive to changes in fluid properties, the arrival time and amplitude of the received signal will vary with the sound speed of the liquid and as a result of attenuation. This mode of operation provides an independent set of measurements compared with pulse-echo operation. 
The same interdigital transducer can be immersed in a fluid half-space and used for measuring the elastic and viscous properties of the medium. For this measurement, a unidirectional Scholte interface wave is generated which propagates from the transmitter toward the receiver, as shown in the following figure.

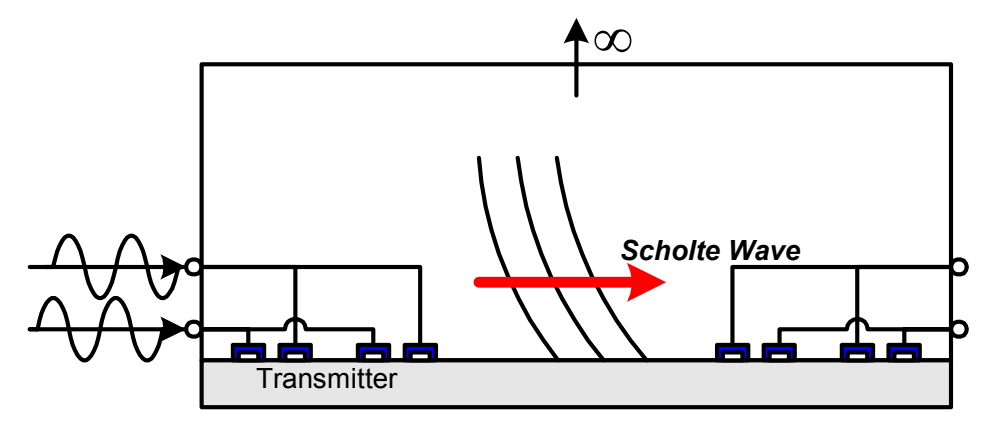

Figure 15 Illustration of interdigital cMUT used for immersion measurements.

This "dip-stick" measurement technique is attractive because the visco-elastic properties of the fluid can be determined using two transducers on a single substrate, separated by a precise, lithographically defined distance.

Interdigital cMUTs can also be used as actuators in microfluidic environments. Exciting the fingers of the transducer with phase shifted, continuous wave signals at the design frequency generates highly directional Scholte waves. When the amplitude of the Scholte wave is large, acoustic streaming forces result in a net flow of the fluid in a particular lateral direction [12]. By changing the relative phase of the signals, bidirectional pumping can be realized. 


\section{CHAPTER 3}

\section{ANALYTICAL MODEL FOR ACOUSTIC MODE PROPAGATION}

To design the interdigital cMUTs, the propagation of acoustic wave modes in various configurations was modeled analytically. These configurations include a free silicon plate, a silicon plate loaded with a fluid half-space, and fluid-filled microchannel. The models were primarily used to determine the geometric dispersion of the phase and group velocities of the propagating modes. Information on phase velocity was needed to design the periodicity of the fingers of the transducer, while knowledge of the group velocity was needed to predict the arrival time of the various modes for experimental validation. The analytical models were also used to determine the mode shapes, field distributions, and sensitivity of modes to changes in material and geometric properties.

For the case of a free, isotropic silicon plate, a closed form solution for the propagating modes can be obtained [36], but this is not the case for the fluid loaded plate and the microchannel. This is because that the solution requires one to evaluate the determinant of a matrix which is formed from the various boundary conditions. For a free plate, there are four boundary conditions which results in a $4 \times 4$ matrix, which can be evaluated analytically with some effort. However, when the plate is coupled to a fluid half-space, the number of boundary conditions increases to five resulting in a $5 \times 5$ matrix which is difficult to invert analytically. For the case of a fluid-filled microchannel with plates of finite thickness, the number of unknowns increases to ten which requires a numerical solution. It should be noted that the following analysis is only valid for an isotropic silicon plate. 
In the following section, the technique used to solve for the propagating modes will be illustrated for the case of a free plate. The results for a free plate, a fluid-loaded plate, and a fluid-filled microchannel will then be presented. The programs written to solve the various problems can be found in Appendix 1 .

\section{$\underline{\text { Free Plate }}$}

Since there is a closed form solution for the modes propagating in a free plate, namely the Rayleigh-Lamb equations, this configuration was used to develop the program for numerically solving for the geometric dispersion of propagating waves. First, the displacement-equation of motion for the solid plate was written in vector notation as [37]:

$$
\mu \nabla^{2} \vec{u}+(\lambda+\mu) \nabla \nabla \cdot \vec{u}=\rho \ddot{\vec{u}}
$$

where $\vec{u}$ is the displacement vector, $\rho$ is the density, $\lambda$ and $\mu$ are the Lame constants, and $\nabla$ is the vector operator $\mathrm{del}$. The displacement vector was then written in terms of scalar, $\phi$, and vector, $\psi$, displacement potentials so that the shear and longitudinal components of the motion could be decoupled:

$$
\vec{u}=\nabla \phi+\nabla \times \vec{\psi}
$$

The use of the vector potentials allows at most two orthogonal motions to be decoupled. In general, there are three orthogonal wave motions possible in a plate. These are longitudinal waves (P-waves), shear vertical waves (SV waves) and shear horizontal waves (SH waves). For this problem, the only modes of interest are the P and SV waves since SH modes do not couple energy to the surrounding fluid. Therefore the use of the vector potentials allows the P and SV waves to be decoupled. 
After substituting the potential representation of the displacement vector (Equation 5) into the equation of motion (Equation 4) and simplifying, one realizes that the equations of motion are satisfied if the following uncoupled wave equations are satisfied:

$$
\begin{aligned}
& \nabla^{2} \phi=\frac{1}{c_{L}^{2}} \ddot{\phi} \\
& \nabla^{2} \psi=\frac{1}{c_{S}^{2}} \ddot{\psi}
\end{aligned}
$$

where

$$
\begin{gathered}
c_{L}^{2}=\frac{\lambda+2 \mu}{\rho} \\
c_{S}^{2}=\frac{\mu}{\rho}
\end{gathered}
$$

Note that $c_{L}$ and $c_{S}$ are the longitudinal and shear wave velocities in an infinite solid medium.

The general solution to the wave equations can then be written as:

$$
\begin{aligned}
& \phi=\left\{\begin{array}{c}
A_{1} \exp \left(j k_{1}^{l} z\right) \\
A_{2} \exp \left(-j k_{1}^{l} z\right)
\end{array}\right\} \exp (j \omega t-\beta x) \\
& \psi=\left\{\begin{array}{c}
A_{3} \exp \left(j k_{1}^{s} z\right) \\
A_{4} \exp \left(-j k_{1}^{s} z\right)
\end{array}\right\} \exp (j \omega t-\beta x)
\end{aligned}
$$

where the \{\} notation indicates that any linear combination of the quantity within the brackets satisfies the wave equation and thus the equations of motion [38]. In these equations, $A_{i}$ are amplitudes, $k_{1}^{l}$ and $k_{1}^{s}$ are wave numbers for the longitudinal and shear modes, $\beta$ is the wave number of the propagating mode, and $\omega$ is the frequency. The wave numbers for the quantities in the brackets are given as: 


$$
\begin{aligned}
& k_{1}^{l}=\sqrt{k_{L}^{2}-\beta^{2}} \\
& k_{1}^{s}=\sqrt{k_{S}^{2}-\beta^{2}}
\end{aligned}
$$

where

$$
\begin{aligned}
& k_{L}=\frac{\omega}{c_{L}} \\
& k_{S}=\frac{\omega}{c_{S}}
\end{aligned}
$$

and

$$
\beta=\frac{\omega}{V_{\text {phase, mode }}}
$$

In Equations 10 and 11 complex exponentials are used for the standing waves in the zdirection even though trigonometric functions could be used. This is done for generality since additional complexity is expected in later problems.

The form of the solution (Equations 10 and 11) is chosen a priori since propagation is expected in the x-direction and standing waves are expected in the zdirection. The geometry of the free plate is shown in the following figure.

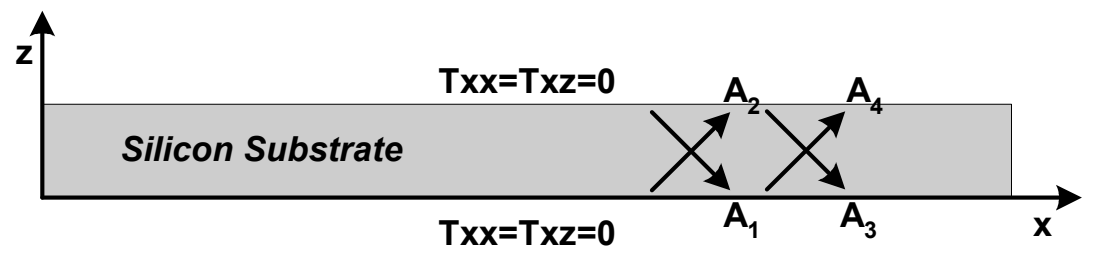

Figure 16 Illustration of coordinate system and boundary conditions for a free plate. 
It is important to note that the length of the plate is assumed to be infinite in the $\mathrm{x}$ direction and finite in the z-direction. The only possible acoustic modes in this twodimensional analysis are $\mathrm{P}$ and $\mathrm{SV}$ waves residing in the $\mathrm{x}-\mathrm{z}$ plane.

To find a particular solution to the wave equation, the boundary conditions for the problem are applied. For the free plate, the boundary conditions require that the normal stress, $T_{z z}$, and shear stress, $T_{x z}$, at the bottom $(z=0)$ and top $\left(z=t_{1}\right)$ surfaces vanish:

$$
\begin{aligned}
& \left.T_{z z}\right|_{z=0}=\left.T_{x z}\right|_{z=0}=0 \\
& \left.T_{z z}\right|_{z=t 1}=\left.T_{x z}\right|_{z=t 1}=0
\end{aligned}
$$

The displacement potentials can be related to the shear and normal stresses through Hooke's law:

$$
\begin{gathered}
T_{x z}=\mu\left(2 \frac{\partial^{2} \phi}{\partial x \partial z}\right)+\mu\left(\frac{\partial^{2} \psi}{\partial z^{2}}\right)-\mu\left(\frac{\partial^{2} \psi}{\partial x^{2}}\right) \\
T_{z z}=(\lambda+2 \mu)\left(\frac{\partial^{2} \phi}{\partial x^{2}}+\frac{\partial^{2} \phi}{\partial z^{2}}\right)-2 \mu\left(\frac{\partial^{2} \phi}{\partial x^{2}}+\frac{\partial^{2} \psi}{\partial x \partial z}\right)
\end{gathered}
$$

After evaluating the various derivatives required by Hooke's Law, one can express the boundary conditions in terms of the displacement potentials. Since there are 4 boundary conditions, one arrives with 4 equations which can be written as a $4 \times 4$ matrix:

$$
\left[\begin{array}{llll}
a_{11} & a_{12} & a_{13} & a_{14} \\
a_{21} & a_{22} & a_{23} & a_{24} \\
a_{31} & a_{32} & a_{33} & a_{34} \\
a_{41} & a_{42} & a_{43} & a_{44}
\end{array}\right]\left\{\begin{array}{l}
A_{1} \\
A_{2} \\
A_{3} \\
A_{4}
\end{array}\right\}=0
$$

In this matrix equation, $a_{i j}$ are the coefficients of the amplitudes resulting from application of the boundary conditions. For brevity, the components of the matrix are not presented. However, they can be found in Appendix 1. Writing the equations in matrix 
form provides some clarity and eases the implementation into the numerical solution program. Since the only unknowns are the amplitudes of the various terms, $A_{i}$, the system of equations can be solved. MATLAB is used for all of the solutions because of its ability to efficiently perform computations with matrices.

The solution proceeds as follows. First, the material properties, frequency, and plate thickness are defined within the main program. The coefficient matrix, $\left[a_{i j}\right]$, is then defined as a subroutine which can be called from the main program. In general, a propagating mode is said to exist when the determinant of the coefficient matrix, $\left[a_{\mathrm{ij}}\right]$, is zero. In practice, it is difficult to find a true zero of the determinant due to numerical scaling issues. Therefore, a mode is said to exist when the absolute value of the determinant of the coefficient matrix reaches an absolute minimum. A subroutine built into MATLAB, fmin, is used to search for the minima of a particular function over a range of values. In this problem, fmin searches over a range of phase velocities for the phase velocity which minimizes the absolute value of the determinant of the coefficient matrix.

To illustrate how the program works, the absolute value of the determinant of the coefficient matrix is plotted against the phase velocity in Figure 17. 


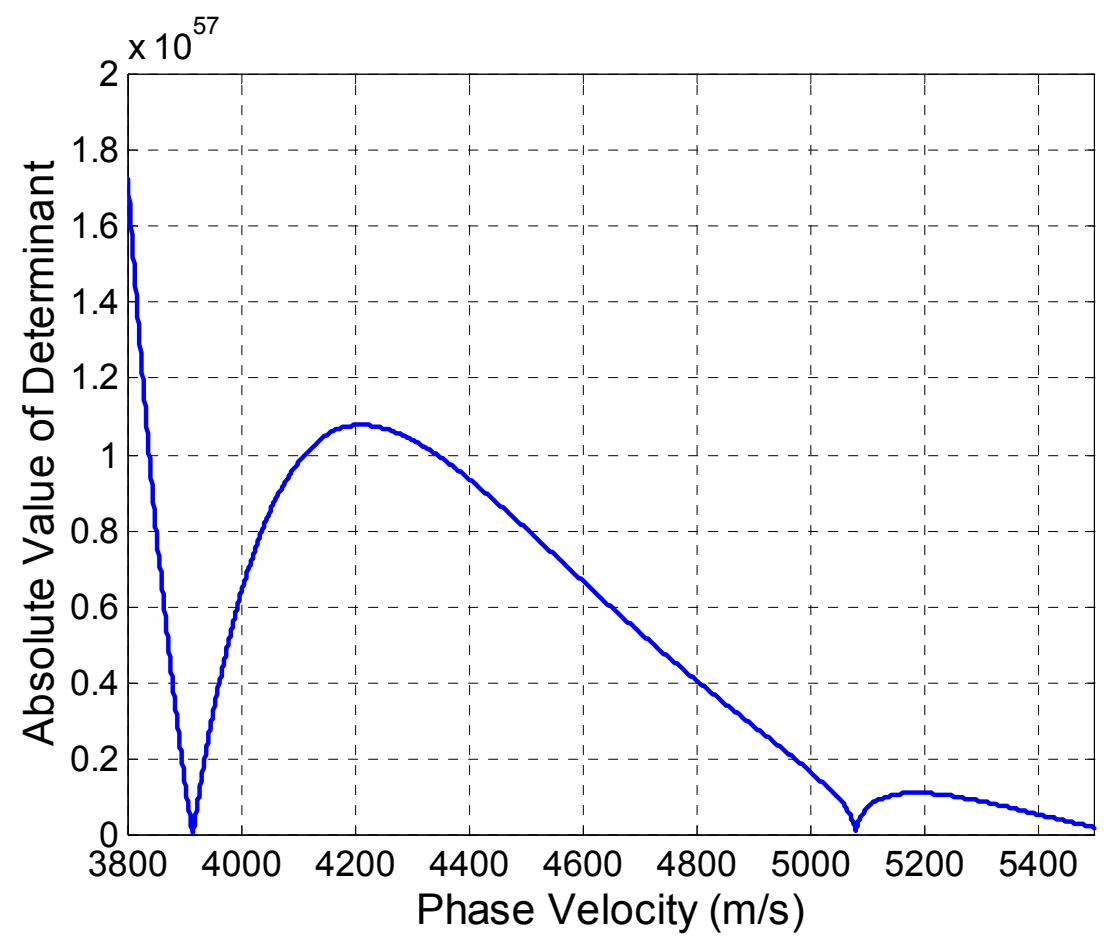

Figure 17 Plot of the determinant of the coefficient matrix calculated as a function of the phase velocity.

One can see that there are absolute minima at phase velocities of approximately 3900 and $5100 \mathrm{~m} / \mathrm{s}$. However, the function fmin cannot determine if the minimum that it finds is absolute or local. For example, if the search range is 4400 to $4600 \mathrm{~m} / \mathrm{s}$, fmin will output that there is a minimum at $4600 \mathrm{~m} / \mathrm{s}$. It is obvious that this is only a local minimum and does not represent a propagating mode. Therefore, an if-structure is used to determine if the phase velocity returned by fmin corresponds to a local or absolute minimum. If the phase velocity returned by fmin corresponds to the starting or ending points of the search range, a mode has not been found. The search parameters are changed and the search is repeated. Once an absolute minimum is found, the phase velocity of this propagating mode is stored, and the search is continued until all of the modes within a particular range 
of interest are found. For most cases, the phase velocity is varied from 1,000 to $10,000 \mathrm{~m} / \mathrm{s}$.

This solution technique was applied to a silicon plate for verification. The longitudinal and shear wave velocities for the silicon were 8412 and $5080 \mathrm{~m} / \mathrm{s}$, respectively. In Figure 18, the phase velocities of the propagating modes are plotted against the frequency-thickness (f-d) product for the plate.

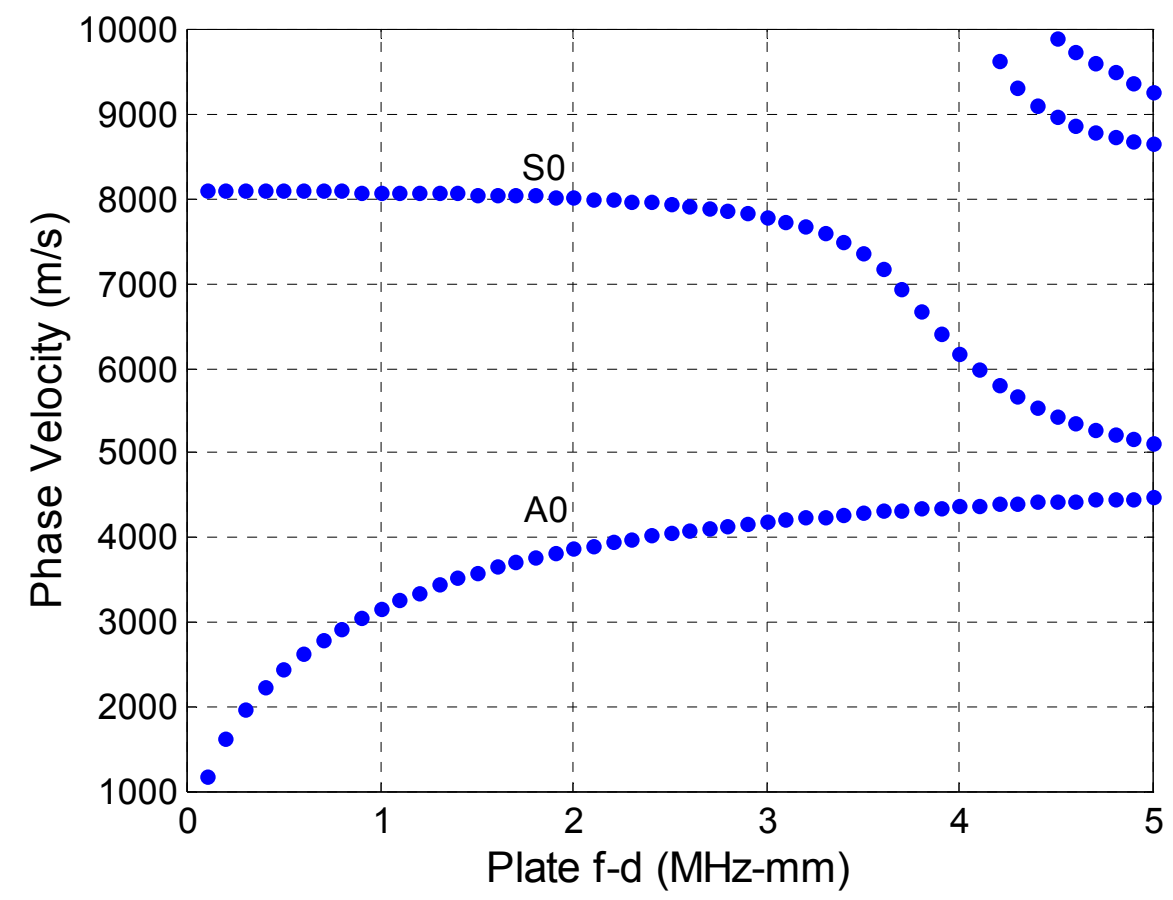

Figure 18 Geometric dispersion of the phase velocity of propagating modes for a free plate.

The results show that there are two dominant modes propagating for $\mathrm{f}$-d values less than $5 \mathrm{MHz}-\mathrm{mm}$ : the zero order symmetric and antisymmetric Lamb waves. The dispersion of these modes matches well with the closed form analytical solution to the Rayleigh-Lamb problem. 
The displacement and stress profiles can be determined using the program used for evaluating the determinant of the coefficient matrix. Since only the shapes and not the amplitudes are of interest, an approach similar to the one used for determining the reflection and transmission coefficients in Acoustics can be employed. One of the coefficients, $A_{i}$, in the particular solution (Equations 10 and 11) is set equal to one. The remaining $A_{\mathrm{i}}$ 's are then written in terms of this newly found known quantity. This allows the fourth order, homogeneous matrix equation (Equation 21) to be written as a third order, non-homogeneous equation:

$$
\left[\begin{array}{lll}
a_{11} & a_{12} & a_{13} \\
a_{21} & a_{22} & a_{23} \\
a_{31} & a_{32} & a_{33}
\end{array}\right]\left\{\begin{array}{l}
A_{1} \\
A_{2} \\
A_{3}
\end{array}\right\}=\left\{\begin{array}{l}
-a_{14} \\
-a_{24} \\
-a_{34}
\end{array}\right\}
$$

The resulting matrix equation (Equation 22) can be easily inverted to produce the remaining amplitude coefficients. Once the amplitude coefficients are know, the displacement and stress fields can be calculated. The displacements in the $\mathrm{x}$ - and $\mathrm{z}-$ directions are calculated from the following:

$$
\begin{aligned}
& u_{x}=\frac{\partial \phi}{\partial x}+\frac{\partial \psi}{\partial z} \\
& u_{z}=\frac{\partial \phi}{\partial z}-\frac{\partial \psi}{\partial x}
\end{aligned}
$$

The stress fields can be calculated using Hooke's Law (Equations 19 and 20).

In the dispersion curve (Figure 16) for an $\mathrm{f}-\mathrm{d}$ of $2 \mathrm{MHz}-\mathrm{mm}$, there are two modes propagating: the zero order symmetric $\left(\mathrm{S}_{0}\right)$ and antisymmetric $\left(\mathrm{A}_{0}\right)$ Lamb waves. The names 'symmetric' and 'antisymmetric' come from the appearance of the x-direction displacement profile for the two modes. For the $\mathrm{S}_{0}$ mode, the $\mathrm{x}$-displacement profile is symmetric about the mid-plane of the plate (Figure 19). 


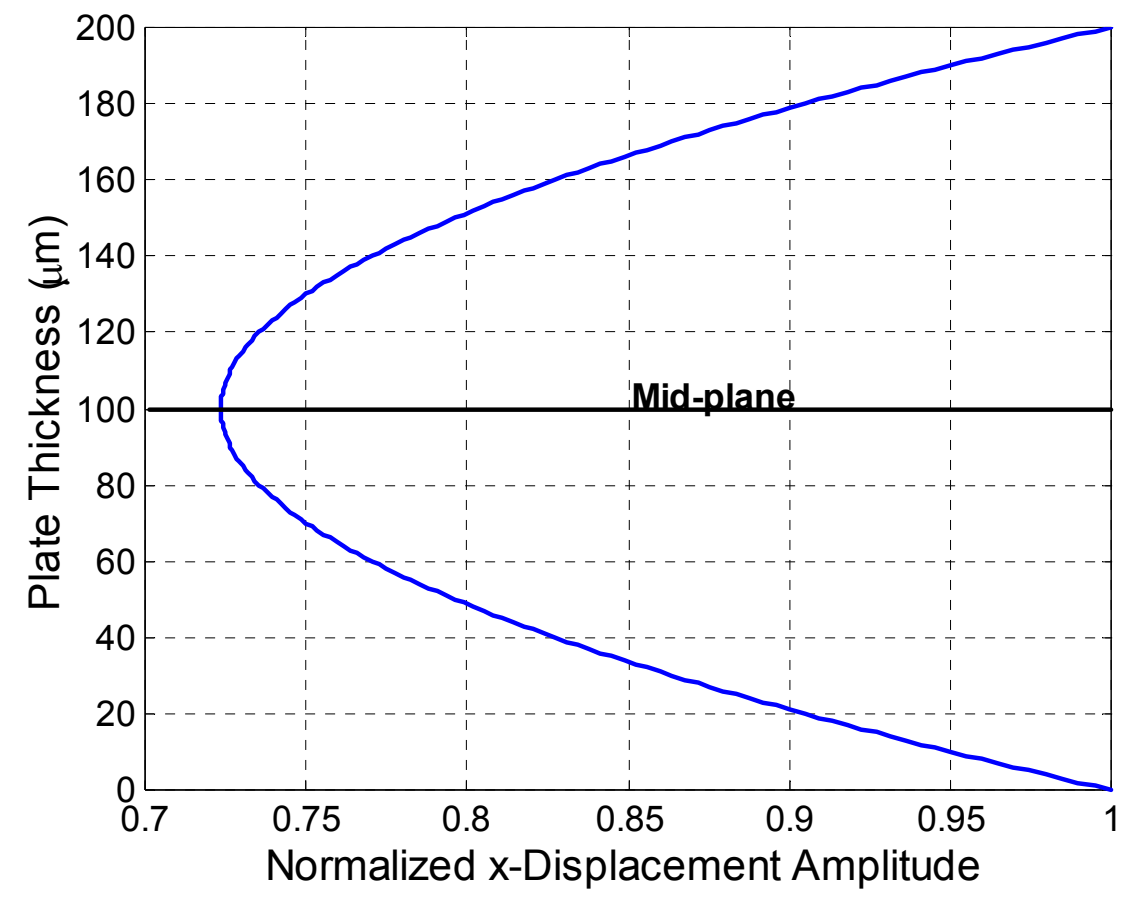

Figure 19 Profile of $x$-direction displacement over the thickness of a $200 \mu \mathrm{m}$ plate at $10 \mathrm{MHz}$ (f-d=2MHzmm). Profile is for the $\mathrm{S}_{0}$ mode with a phase velocity of $8018 \mathrm{~m} / \mathrm{s}$.

For the $\mathrm{A}_{0}$ mode, the displacement profile is antisymmetric about the mid-plane (Figure 20). 


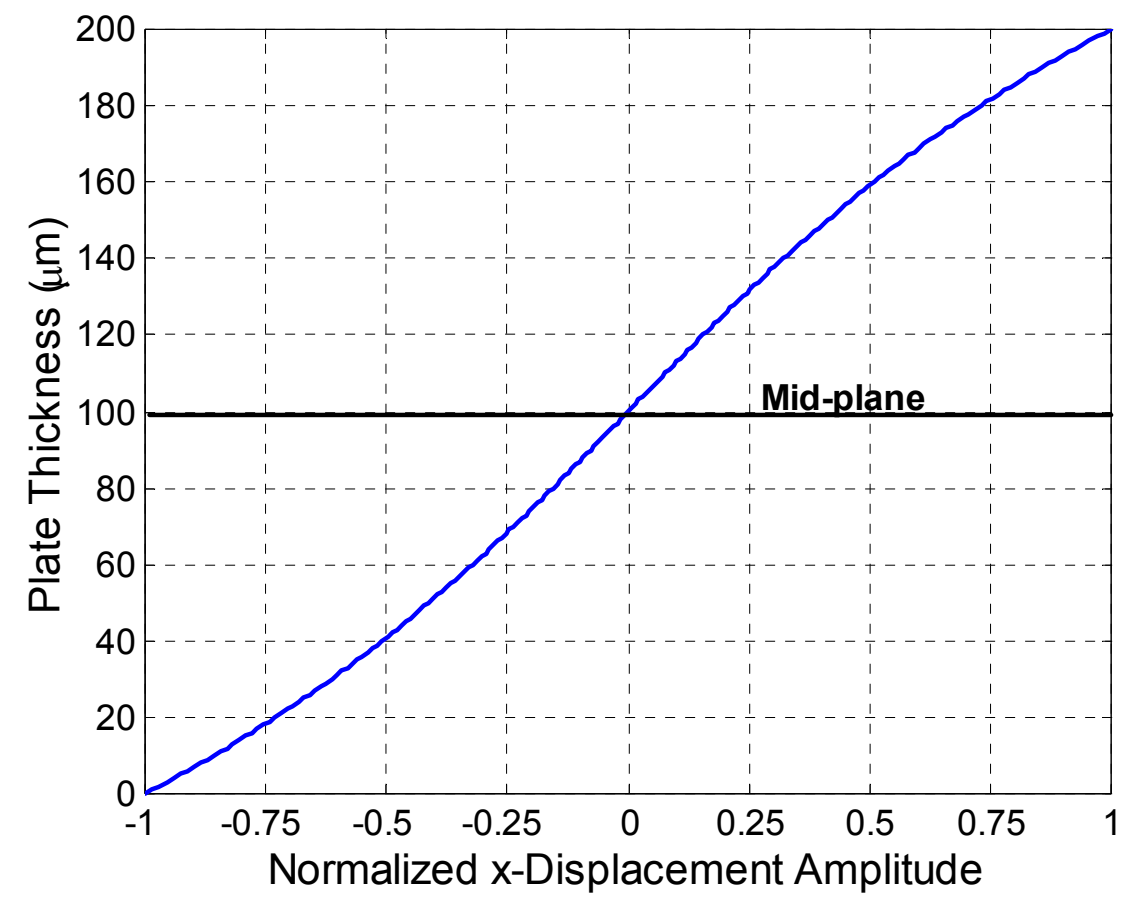

Figure 20 Profile of $x$-direction displacement over the thickness of a $200 \mu \mathrm{m}$ plate at $10 \mathrm{MHz}(\mathrm{f}-\mathrm{d}=2 \mathrm{MHzmm})$. Profile is for the $\mathrm{A}_{0}$ mode with a phase velocity of $3866 \mathrm{~m} / \mathrm{s}$.

After validating the model for the geometric dispersion of propagating modes in the finite plate, the complexity of the model could be increased to involve fluid loading. 


\section{Fluid Loaded Plate}

The silicon plate was coupled to a semi-infinite fluid half-space to determine which modes could propagate in this configuration. The general solution of the wave equation in the fluid has the following form:

$$
\phi_{f}=\left\{\begin{array}{c}
A_{5} \exp \left(j k_{f} z\right) \\
A_{6} \exp \left(-j k_{f} z\right)
\end{array}\right\} \exp (j \omega t-\beta x)
$$

where $\phi_{f}$ is the displacement potential, and $k_{f}$ is the wave number in the fluid. It is important to note that there is only a scalar displacement potential because it is assumed that the fluid cannot support any shear motion. Since it is assumed that the fluid extends to infinity in the positive z-direction, there can be no waves propagating in the negative zdirection (toward the plate) in the fluid; this forces $A_{6}$ to zero. This constitutes the causality condition.

A schematic of the geometry and boundary conditions is shown in Figure 21.

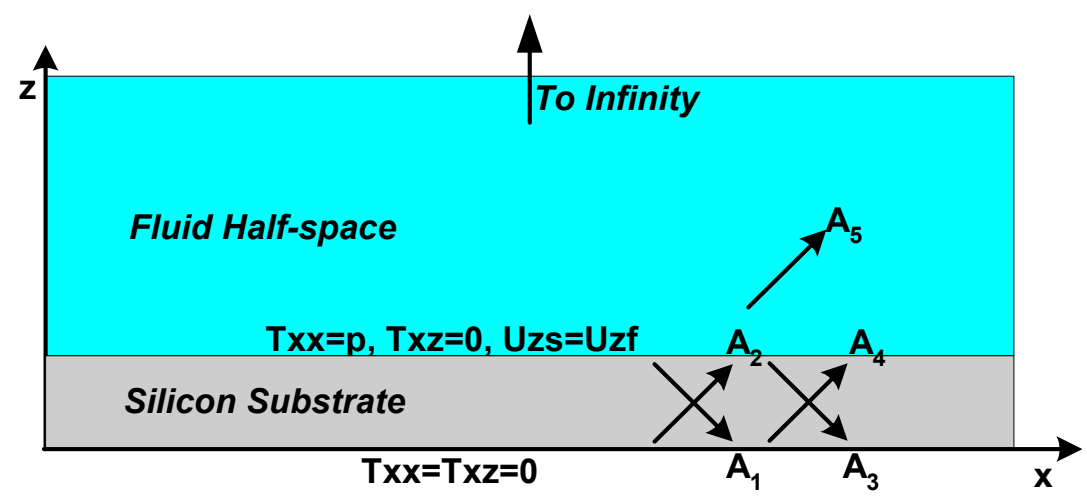

Figure 21 Illustration of coordinate system and boundary conditions for a fluid-loaded plate. 
The boundary conditions for the bottom of the plate $(z=0)$ remain the same: stress free. However, the boundary conditions at the interface of the solid and fluid were changed to reflect the coupling at the interface. The continuity boundary conditions at the top of the plate are as follows:

$$
\begin{gathered}
T_{x z}=0 \\
T_{z z}=p \\
u_{z, \text { solid }}=u_{z, \text { fluid }}
\end{gathered}
$$

The shear stress, $T_{\mathrm{xz}}$, at the interface remained zero since it is assumed that the fluid cannot support shear stresses. The second boundary condition requires that the normal stress, or pressure, across the interface be constant. If the liquid is modeled as a solid with no viscosity, the pressure in the fluid can be calculated from Hooke's Law by setting $\mu$ and $\psi$ equal to zero:

$$
p=\lambda\left(\frac{\partial^{2} \phi}{\partial x^{2}}+\frac{\partial^{2} \phi}{\partial z^{2}}\right)
$$

Finally, the normal motion of the fluid and solid was forced to be continuous across the interface.

After applying the boundary conditions using Hooke's law, one arrives with a $5 \times 5$ homogeneous matrix equation. The same technique used to solve for the modes in the plate can be used for finding the modes that can propagate in a fluid-loaded plate. Solving for the propagating modes over a range of plate thickness-frequency products produces the geometric dispersion curve for the system. In addition to symmetric and antisymmetric Lamb waves, a third mode was found to exist: the Scholte wave [23]. As previously stated, the Scholte wave is an interface wave, similar to a Stoneley wave [22], 
which exists at the interface of a fluid and a solid. The geometric dispersion of the Scholte wave is shown in Figure 22. The phase velocity of the Scholte wave is always less than the sound speed in both the fluid and the solid. For example, when the sound speed in the fluid is $1500 \mathrm{~m} / \mathrm{s}$, the maximum Scholte wave phase velocity is $1499.25 \mathrm{~m} / \mathrm{s}$. The subsonic phase velocity of the Scholte wave results in a mode which is evanescent and therefore does not radiate energy into the surrounding fluid. The wave propagates laterally along the fluid-solid interface without any diminution in amplitude. Only material losses can remove energy from the mode.

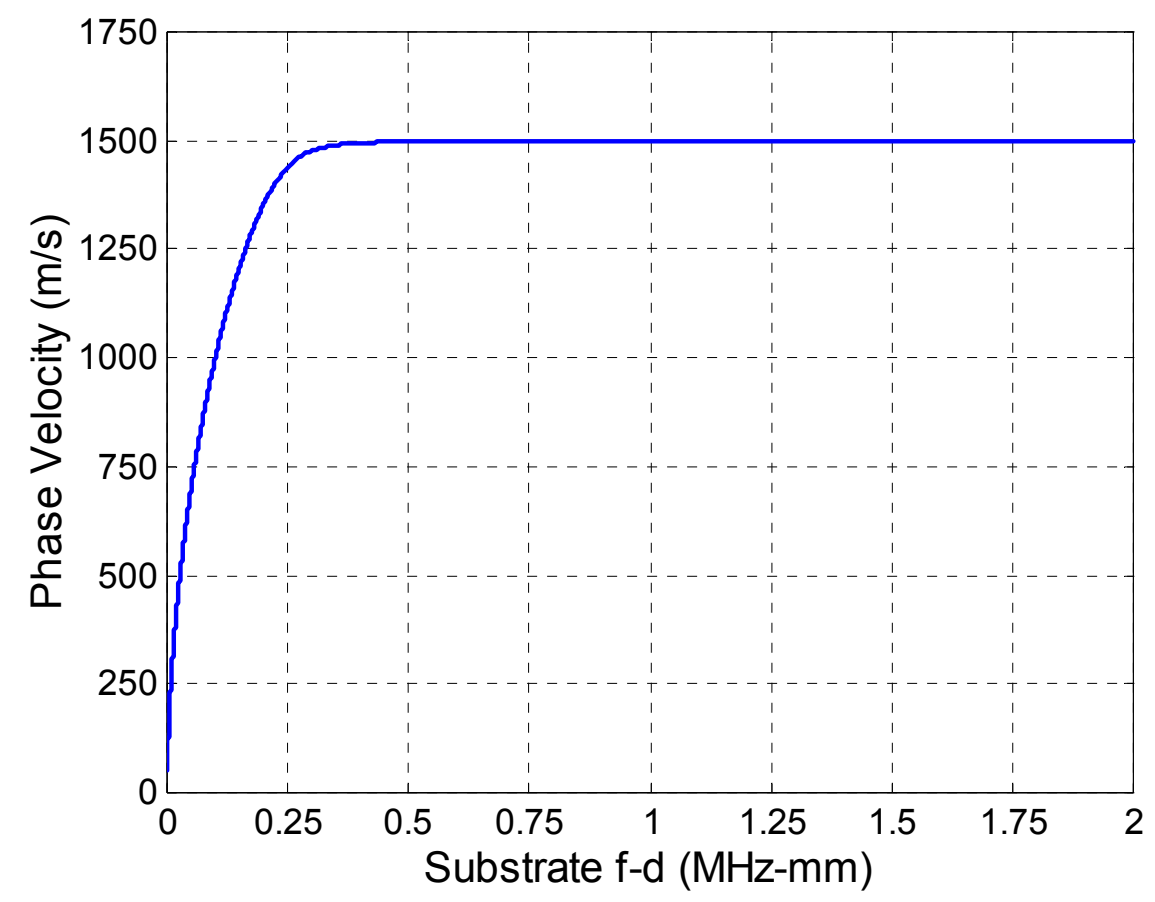

Figure 22 Geometric dispersion of the Scholte wave for a fluid half-space attached to a silicon plate.

By setting the amplitude coefficient $A_{5}$ equal to 1 , one can solve for the displacement fields for the modes of interest. The mode shape of the Scholte wave is shown in Figure 23. 


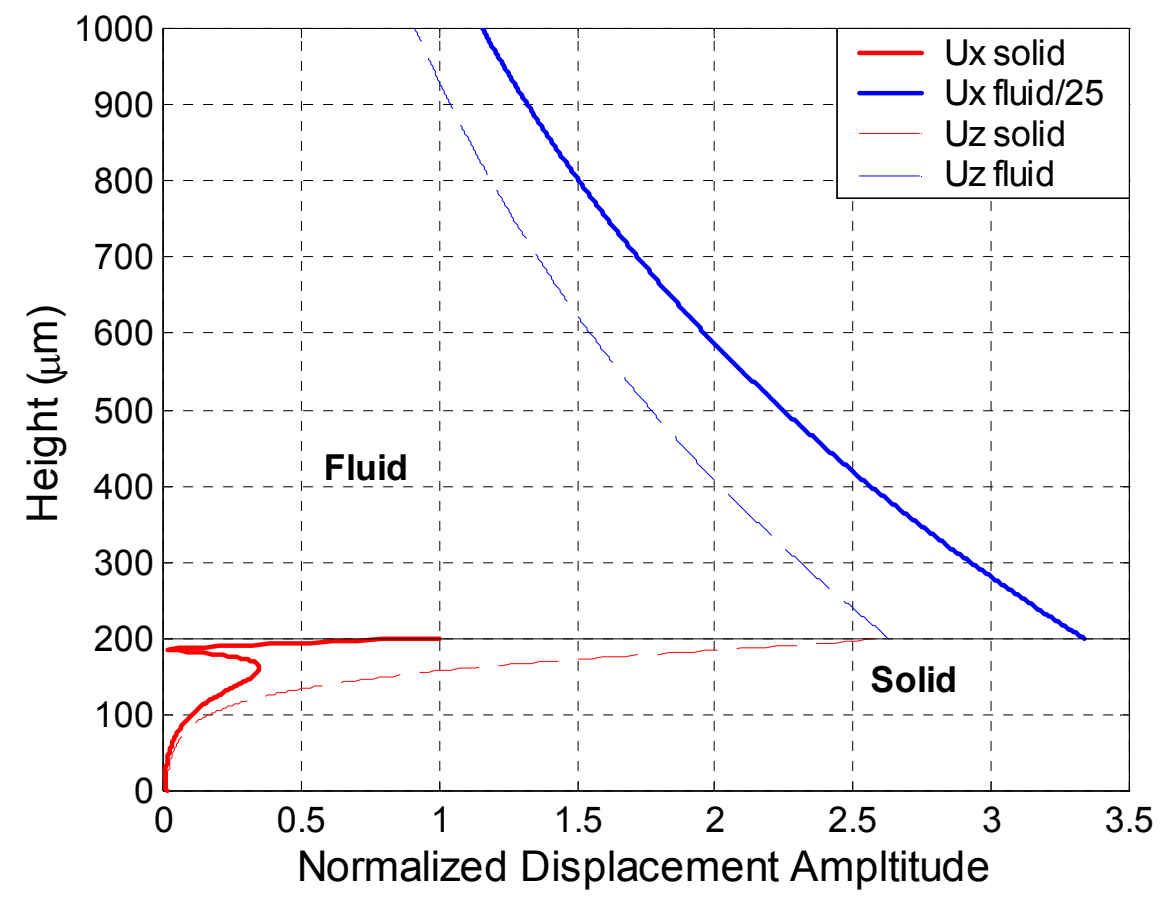

Figure 23 Displacement field for the Scholte wave at the interface of a water half-space and a $200 \mu \mathrm{m}$ plate at $10 \mathrm{MHz}$.

It is important to note that the $\mathrm{x}$-displacement in the fluid is more than 25 times greater than the $\mathrm{x}$-displacement in the solid. This is indicative of the energy concentration of the Scholte wave which has the majority of the energy in the fluid.

Knowledge of the mode shapes allows one to calculate the rate of decay of the $\mathrm{x}-$ displacement amplitude as a function of distance from the plate. This decay rate was found to be $-1.15 \mathrm{~dB} /(\mathrm{MHz}-\mathrm{mm})$, when normalized to the plate $\mathrm{f}-\mathrm{d}$. So, for a frequency of $10 \mathrm{MHz}$, the amplitude of the $\mathrm{x}$-displacement of the Scholte wave decreases $11.5 \mathrm{~dB}$ in $1 \mathrm{~mm}$ of vertical distance from the plate.

The relative amounts of energy contained in the fluid and the solid can be obtained by integrating the product of the stress and pressure along a line extending in the z-direction. For the solid, the power in the $\mathrm{x}$-direction is given by: 


$$
P_{x, \text { Plate }}=\frac{1}{2} \operatorname{Re}\left[j \omega\left(T_{x x} \cdot u_{x}^{*}+T_{x z} \cdot u_{z}^{*}\right)\right]
$$

where $u_{x}^{*}$ and $u_{z}^{*}$ are the complex conjugates of the $\mathrm{x}$ - and $\mathrm{z}$-displacements in the solid. The $j \omega$ term comes from taking the time derivative of the particle displacement to get the particle velocity. The power in the fluid has the following similar, but simpler, form:

$$
P_{x, \text { Fluid }}=\frac{1}{2} \operatorname{Re}\left[j \omega\left(p_{\text {Fluid }} \cdot u_{x, \text { Fluid }}^{*}\right)\right]
$$

where $p_{\text {Fluid }}$ is the pressure in the fluid and $u_{x, \text { Fluid }}^{*}$ is the complex conjugate of the $\mathrm{x}$ direction particle displacement in the fluid. By integrating Equations 30 and 31 along the z-direction, one finds that nearly $99 \%$ of the energy in the Scholte wave exists in the fluid when the plate $\mathrm{f}-\mathrm{d}$ is greater than approximately $0.5 \mathrm{MHz}-\mathrm{mm}$. This makes the Scholte wave very sensitive to changes in fluid properties and attractive for microfluidic applications. As the substrate f-d is decreased from $0.5 \mathrm{MHz}-\mathrm{mm}$ (i.e., for thin plates or low frequencies), an increasing amount of power in the Scholte mode is confined to the substrate making the mode less attractive for fluid sensing.

An important result of the analytical model for the plate attached to a fluid-halfspace is the Scholte wave dispersion curve (Figure 22). The dispersion curve predicts that the Scholte wave is essentially non-dispersive for a plate $\mathrm{f}-\mathrm{d}$ of over $1 \mathrm{MHz}-\mathrm{mm}$. For a $500 \mu \mathrm{m}$ thick silicon plate, this corresponds to a frequency of $2 \mathrm{MHz}$. Therefore, when designing Scholte wave transducers which operate at frequencies above $2 \mathrm{MHz}$, it is not necessary to take into account the dispersive nature of the Scholte wave. 


\section{Fluid Filled Microchannel}

For microfluidic applications, the fluid will be confined to a channel formed between two solid plates. The complexity of the analytical model was increased to determine which acoustic modes could propagate in this configuration. The model of the microfluidic channel consists of two $200 \mu \mathrm{m}$ silicon plates separated by a water filled gap of variable height. The substrate thickness was fixed at $200 \mu \mathrm{m}$ so that only the lowest order Lamb waves could propagate. The boundary conditions are geometry are shown in Figure 24.

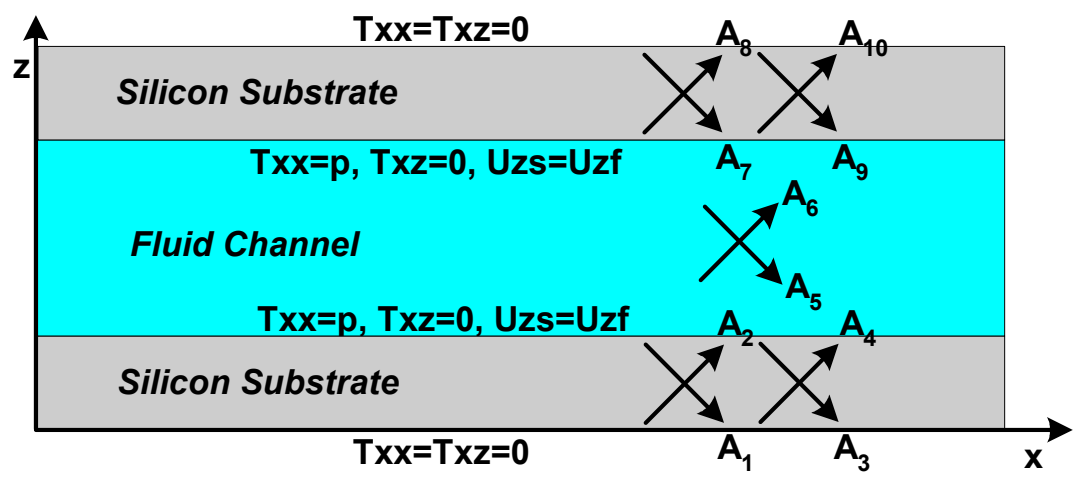

Figure 24 Illustration of coordinate system and boundary conditions for a fluid-filled microchannel.

The two free-surfaces of the silicon plates have traction free boundary conditions, and the fluid-solid interfaces require continuity of normal stress and displacement. The general solution for the modes in the fluid region is given by:

$$
\phi_{f}=\left\{\begin{array}{c}
A_{5} \exp \left(j k_{f} z\right) \\
A_{6} \exp \left(-j k_{f} z\right)
\end{array}\right\} \exp (j \omega t-\beta x)
$$

For the fluid half-space model, the causality boundary condition forced the coefficient of the wave propagating in the negative z-direction, $A_{6}$, to be zero. However, for the case of 
the fluid channel, waves can propagate in the fluid in the positive and negative zdirections. To make the program as general as possible, it was assumed that the top plate had different material properties than the bottom plate. However, the following results are presented for two plates with identical material properties so that the guided modes in the channel could be more easily identified.

Varying the channel height while holding the frequency fixed at $10 \mathrm{MHz}$ produces a dispersion curve for the phase velocity of the propagating modes as seen in Figure 25.

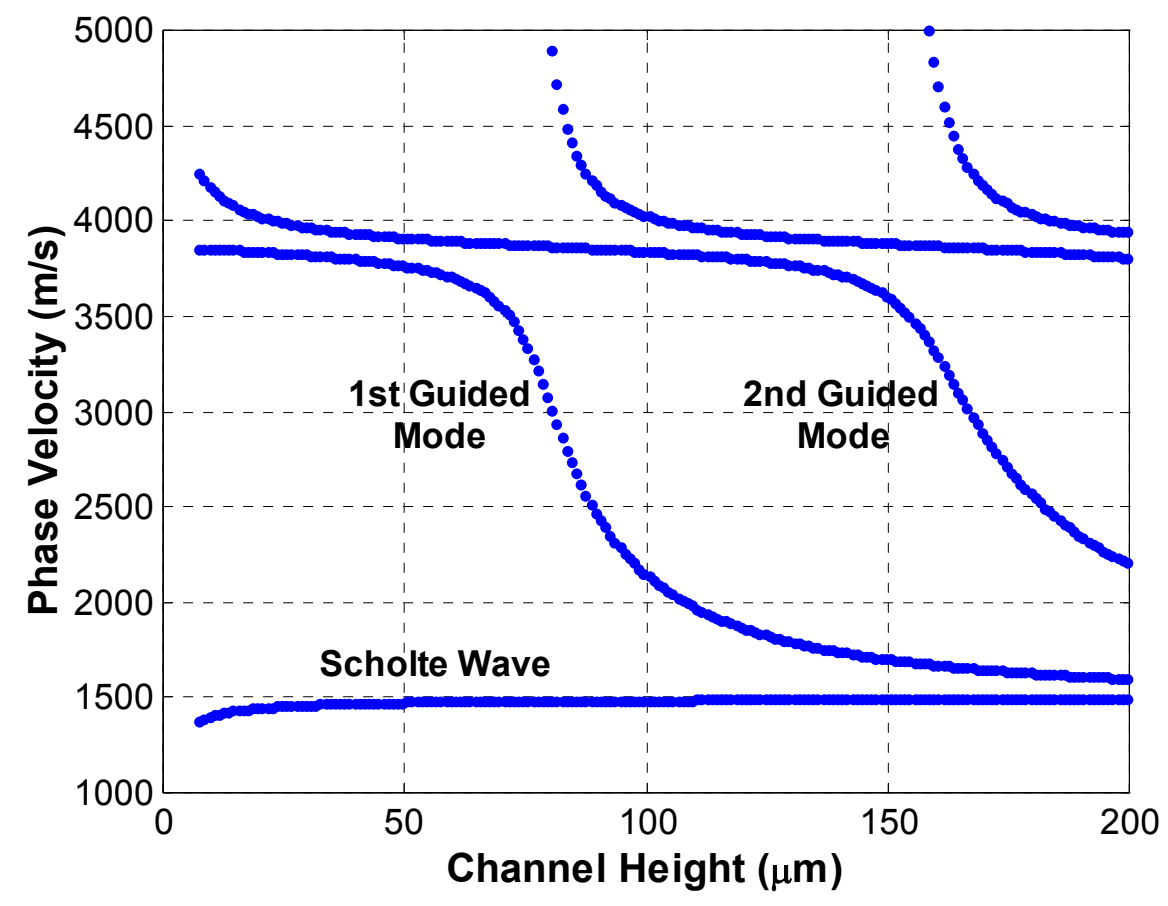

Figure 25 Dispersion of phase velocity for propagating modes as a function of the channel height in a water filled channel at $10 \mathrm{MHz}$. The top and bottom substrates are made of $200 \mu \mathrm{m}$ thick silicon plates.

In addition to lowest order Lamb waves, Scholte waves and other guided modes can propagate in this structure. The Scholte wave in the channel is essentially non-dispersive for channel heights greater than $50 \mu \mathrm{m}$ (at $10 \mathrm{MHz}$ ). For a channel height of $100 \mu \mathrm{m}$, 
modes can propagate with phase velocities of: $1488.7,2152.0,3840.1$, and $4029.2 \mathrm{~m} / \mathrm{s}$. These modes can be identified as the Scholte wave, the first guided channel mode, the second guided channel mode, and the $\mathrm{A}_{0}$ Lamb wave, respectively. The $\mathrm{x}$-displacement profiles of the Scholte wave and first guided channel mode are shown in Figure 26 and Figure 27, respectively.

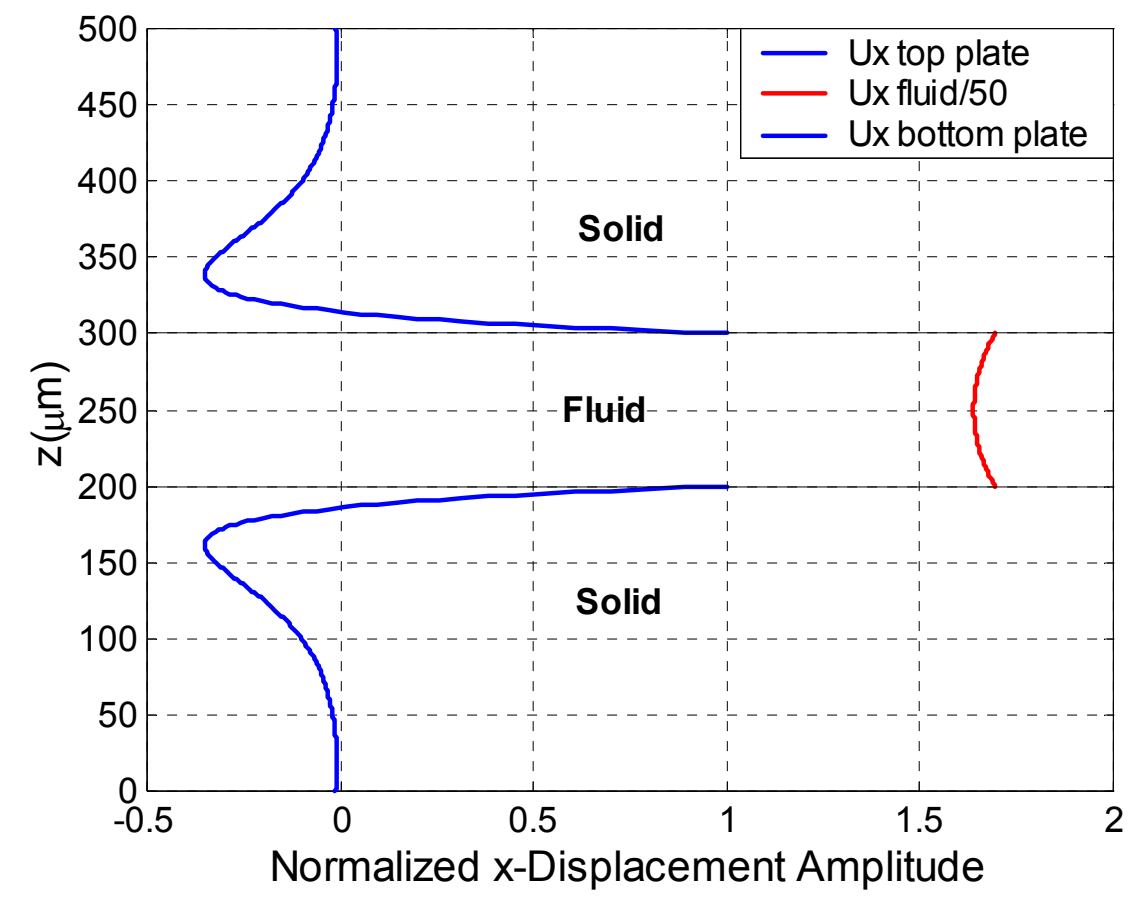

Figure 26 Profile of displacement in the $\mathrm{x}$-direction for the Scholte wave in a $100 \mu \mathrm{m}$ channel at $10 \mathrm{MHz}$. Note that the displacement in the fluid is more than 50 times greater than the displacement in the plates. 


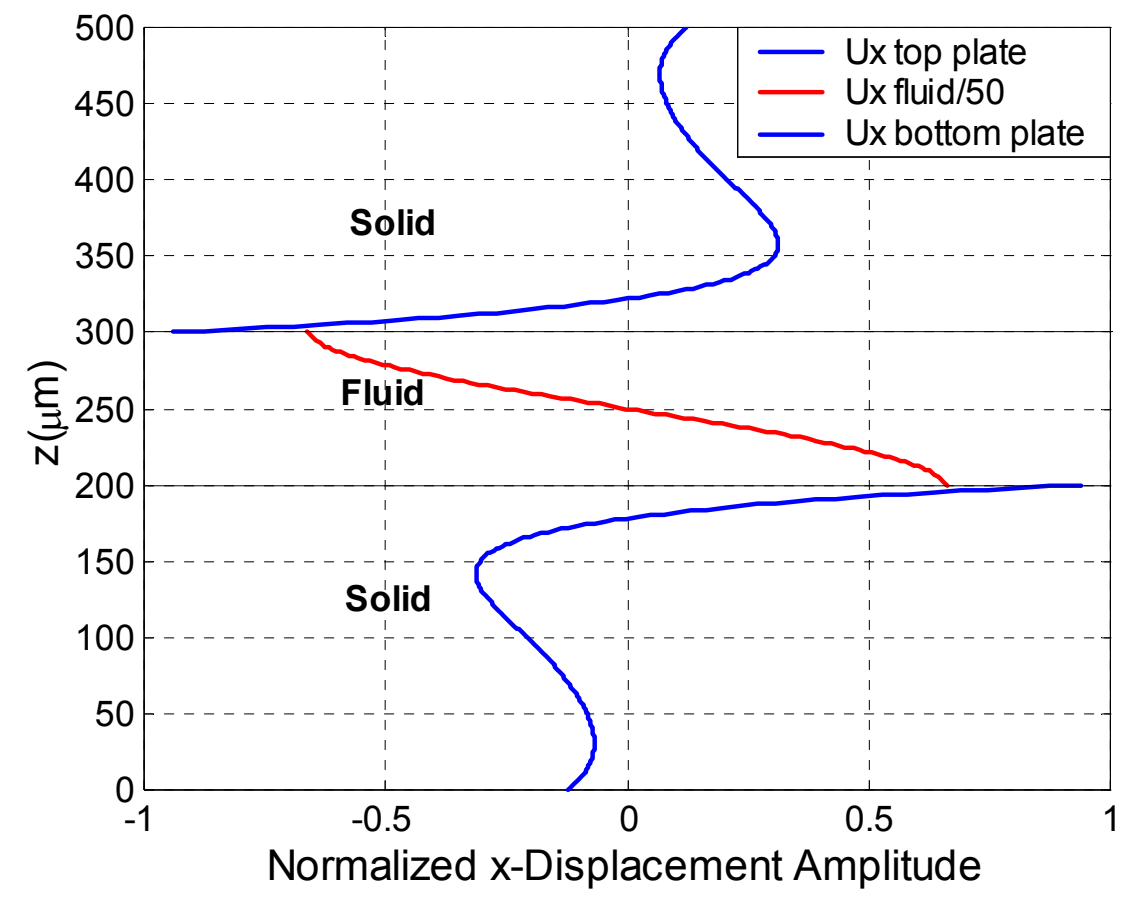

Figure 27 Profile of displacement in the x-direction for the first guided mode in a $100 \mu \mathrm{m}$ channel at $10 \mathrm{MHz}$.

The Scholte wave and the first guided channel mode have most of their energy concentrated in the fluid, $97.6 \%$ and $92.1 \%$ respectively, and are thus promising sensing and actuation tools for microfluidic applications. The second guided channel mode and the $A_{0}$ Lamb wave have the majority of their energy in the silicon plates and are not very attractive for microfluidic applications.

The group velocity ( $\left.\mathrm{V}_{\text {group }}\right)$, or the velocity at which energy propagates, is another quantity of interest and is defined as

$$
V_{\text {group }}=\frac{\partial \omega}{\partial \beta}
$$

where 


$$
\beta=\frac{\omega}{V_{\text {phase }}}
$$

The group velocity is important for experimental purposes because it can be used to predict the arrival time of a particular mode for time-domain measurements. The group velocities of the propagating modes were determined by calculating the phase velocities of the propagating modes over a range of channel heights at two close frequencies: 10 and $10.0001 \mathrm{MHz}$. The differential definition of the group velocity was approximated as follows and was used to calculate the group velocity at each point:

$$
V_{\text {group }}=\frac{\Delta \omega}{\Delta \beta}
$$

The resulting dispersion of the group velocity is shown in Figure 28.

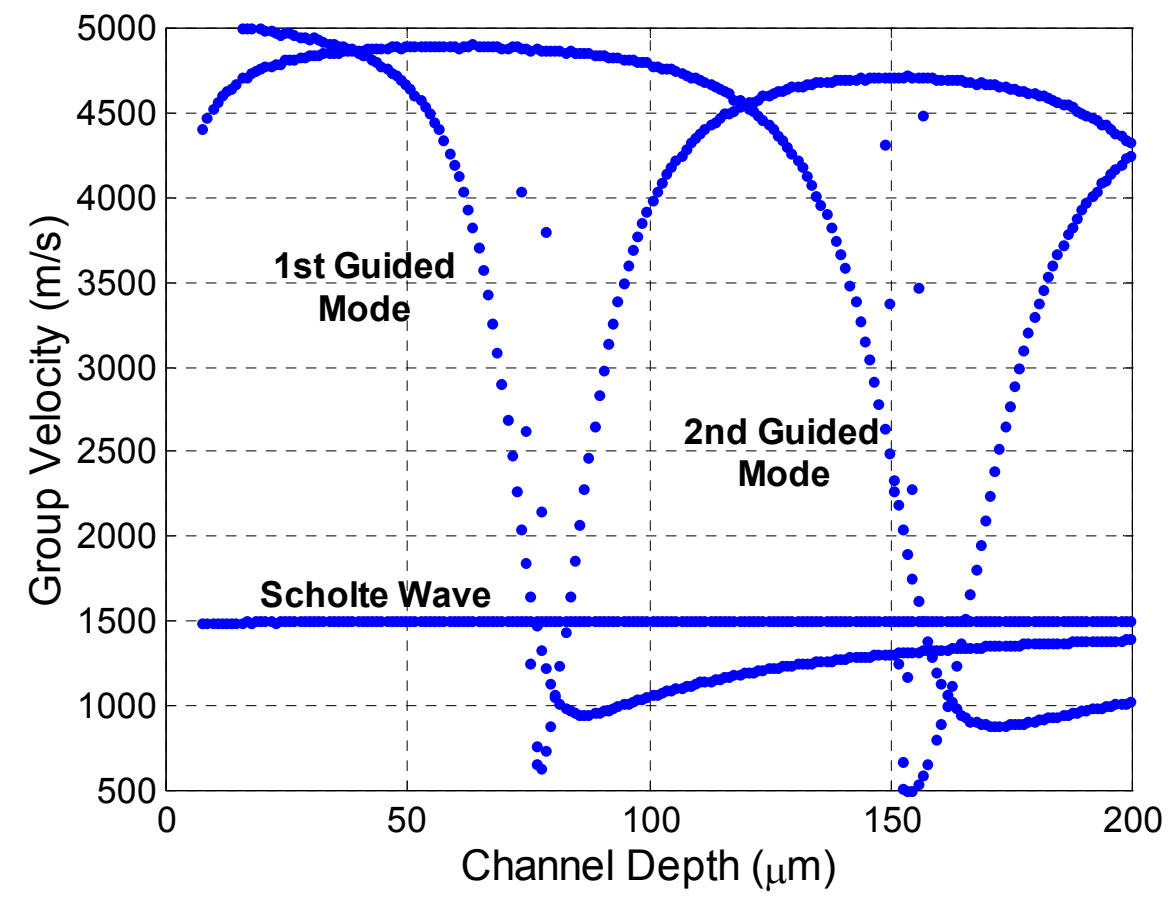

Figure 28 Dispersion of group velocity for modes propagating in a water filled channel of variable height at $10 \mathrm{MHz}$. The plates forming the channel are $200 \mu \mathrm{m}$ thick silicon. 
One should note that the Scholte wave is essentially non-dispersive, but the guided channel modes are highly dispersive. The arrival times of the guided waves could thus be used for monitoring the height of the microchannel.

The analytical model for the channel was also used to determine the sensitivity of the propagating modes to changes in material and geometric properties. The sensitivities were calculated by incrementally changing a single geometric or material property and calculating the resulting change in phase velocity, $V_{\text {phase, }}$, of the various modes. The sensitivity, $S$, to changes in fluid sound speed, $c_{\text {fluid }}$, is defined as

$$
S_{c_{\text {fluid }}}=\frac{d V_{\text {phase }}}{d c_{\text {fluid }}} .
$$

and has units of $(\mathrm{m} / \mathrm{s})(\mathrm{m} / \mathrm{s})^{-1}$. Similarly, the sensitivities of the phase velocity of a particular mode to changes in substrate shear and longitudinal wave speeds, $c_{\text {shear }}$ and $c_{\text {long, }}$, are defined as

$$
S_{c_{\text {shear }}}=\frac{d V_{\text {phase }}}{d c_{\text {shear }}}
$$

and

$$
S_{c_{\text {ong }}}=\frac{d V_{\text {phase }}}{d c_{\text {long }}} .
$$

The modes were also found to be somewhat sensitive to changes in the density of the substrate and the fluid. The sensitivity of the phase velocity to changes in density is defined as

$$
S_{\rho}=\frac{d V_{\text {phase }}}{d \rho}
$$


where $\rho$ is the density of the fluid or solid; the units of $S_{\rho}$ are $(\mathrm{m} / \mathrm{s})\left(\mathrm{kg} / \mathrm{m}^{3}\right)^{-1}$. From the dispersion curve of Figure 25, it is obvious that the phase velocity of the propagating modes is highly dependent on the channel height. This is especially true for the guided channel modes. The sensitivity of the phase velocity to changes in channel height, $h$, is defined as

$$
S_{\text {height }}=\frac{d V_{\text {phase }}}{d h}
$$

and has units of $(\mathrm{m} / \mathrm{s}) \mu \mathrm{m}^{-1}$. The sensitivity of the first three modes in a $100 \mu \mathrm{m}$ waterfilled channel at $10 \mathrm{MHz}$ are presented in Table 1.

Table 1 Sensitivity of first three guided modes in a $100 \mu \mathrm{m}$ channel at $10 \mathrm{MHz}$ to changes in material and geometric parameters.

\begin{tabular}{|c|c|c|c|c|c|c||}
\hline $\begin{array}{c}\text { Mode } \\
\left(\mathrm{V}_{\text {phase }}(\mathrm{m} / \mathrm{s})\right)\end{array}$ & $S_{c_{\text {fluid }}}$ & $S_{c_{\text {shear }}}$ & $S_{c_{\text {long }}}$ & $S_{\rho_{\text {fluid }}}$ & $S_{\rho_{\text {solid }}}$ & $S_{\text {height }}$ \\
\hline $\begin{array}{c}\text { Scholte } \\
(1486 \mathrm{~m} / \mathrm{s})\end{array}$ & 0.97 & $2.5 \mathrm{e}-3$ & $-1.4 \mathrm{e}-3$ & -0.011 & $-3.5 \mathrm{e}-4$ & 0.106 \\
\hline $\begin{array}{c}1^{\text {st }} \mathrm{Guided} \\
(2144 \mathrm{~m} / \mathrm{s})\end{array}$ & 2.74 & $2.6 \mathrm{e}-2$ & $-1.3 \mathrm{e}-2$ & -0.101 & $-7.86 \mathrm{e}-3$ & -21.5 \\
\hline $\begin{array}{c}2^{\text {nd }} \mathrm{Guided} \\
(3840 \mathrm{~m} / \mathrm{s})\end{array}$ & 0.10 & 0.56 & -0.17 & -0.026 & -0.617 & -1.54 \\
\hline
\end{tabular}

From the values in Table 1, one can see that the second guided mode is very sensitive to changes in solid properties but not to changes in fluid properties. This is expected since this mode is similar to lowest order antisymmetric $\left(\mathrm{A}_{0}\right.$ mode) Lamb waves in the silicon substrate. However, the first guided channel mode is very sensitive to changes in channel height, with a sensitivity of $-21.5^{\mathrm{m} / \mathrm{s}} / \mu \mathrm{m}$. From the dispersion curve for phase velocity (Figure 25), one can see that the first guided mode is most sensitive to changes in channel height for channel thicknesses of around $80 \mu \mathrm{m}$. At this point, the sensitivity of the mode 
to changes in channel height is approximately $-70 \mathrm{~m} / \mathrm{s} / \mu \mathrm{m}$. The first channel mode is also very sensitive to changes in fluid sound speed.

The calculations show that the phase velocity ( $\left.\mathrm{V}_{\text {phase }}\right)$ of the Scholte wave is very sensitive to changes in propagation speed in the fluid, $\left(c_{f}\right), d_{\text {phase }} / \mathrm{dc}_{\text {fluid }} \sim 1$, but insensitive to changes in solid properties, $\mathrm{dV}_{\text {phase }} / \mathrm{dc}_{\text {shear }} \sim 0.003$. Therefore this propagating mode is suitable for selectively sensing the properties of the fluid.

Based on the results of the analytical model, interdigital cMUTs were designed to generate Scholte waves in fluid half-spaces and microchannels. Since the phase velocity of the mode was non-dispersive over the frequency range of interest, 5 to $20 \mathrm{MHz}$, the periodicity of the transducer, $\lambda_{\text {transducer }}$ was easily calculated from:

$$
\lambda_{\text {transducer }}=\frac{V_{\text {phase,Scholte }}}{f}
$$

where $V_{\text {phase,Scholte }}$ is the phase velocity of the Scholte wave and $f$ is the excitation frequency. Transducers were designed for operation at 5,10 , and $20 \mathrm{MHz}$ and the periodicity of the individual membranes was determined for each frequency. The values are summarized in Table 2.

Table 2 Periodicity of Scholte wave transducers designed for operation in water halfspaces and microchannels.

\begin{tabular}{|l|c|c|c||}
\hline & $\mathbf{5 M H z}$ & $\mathbf{1 0 M H z}$ & $\mathbf{2 0 M H z}$ \\
\hline$\lambda$ Transducer & $292 \mu \mathrm{m}$ & $146 \mu \mathrm{m}$ & $73.5 \mu \mathrm{m}$ \\
\hline
\end{tabular}

Other guided modes can be generated with the same transducers by changing the excitation frequency such that the finger periodicity matches the wavelength of the mode to be generated, or vice versa. 


\section{CHAPTER 4}

\section{COUPLED-FIELD FINITE ELEMENT MODEL OF CMUT ARRAYS}

The analytical model was able to determine which modes could propagate in various configurations based on the geometry and boundary conditions. However, the model was not able to take into account any initial or source conditions corresponding to the membranes of the cMUT array. To determine the effect of membrane size, spacing and phasing on acoustic mode generation and directionality, the transducers were modeled using the finite element program ANSYS (ANSYS, Inc, Canonsburg, PA). A two-dimensional, harmonic acoustic analysis was performed to model the wave propagation in both the solid and the liquid [39]. The simulations make use of the multiphysics capabilities of ANSYS in which structural disturbances in the solid are coupled to the motion of the fluid, and vice-versa. For the analysis, it is assumed that linear acoustics apply and that the fluid is inviscid and lossless. 


\section{Development of Finite Element Model}

Before modeling cMUT arrays, the propagation of acoustic waves in both the fluid and solid was verified. For the acoustic analysis, radiation from a baffled piston, which has a well know closed-form solution, served as the test case for developing the model of acoustic radiation from a vibrating body into the surrounding fluid. For wave propagation in elastic solids, a silicon plate was simulated and excited to generate Lamb waves. In the following section, the development of the various aspects of the finite element model is presented. Then, the models and analysis results for an interdigital cMUT array are presented.

\section{Analysis of Baffled Piston}

To verify that the acoustic simulation module in ANSYS worked properly, the radiation from a baffled piston was modeled. This problem has a well know closed form solution that can be used to verify the FEM results. The finite element model consists of four major components: the solid structure, the fluid, the fluid-structure interface (FSI), and the absorbing boundary. The geometry for the baffled piston simulation and the major components of the model are illustrated in Figure 29. The circular piston was implemented using an axisymmetric finite element model. 


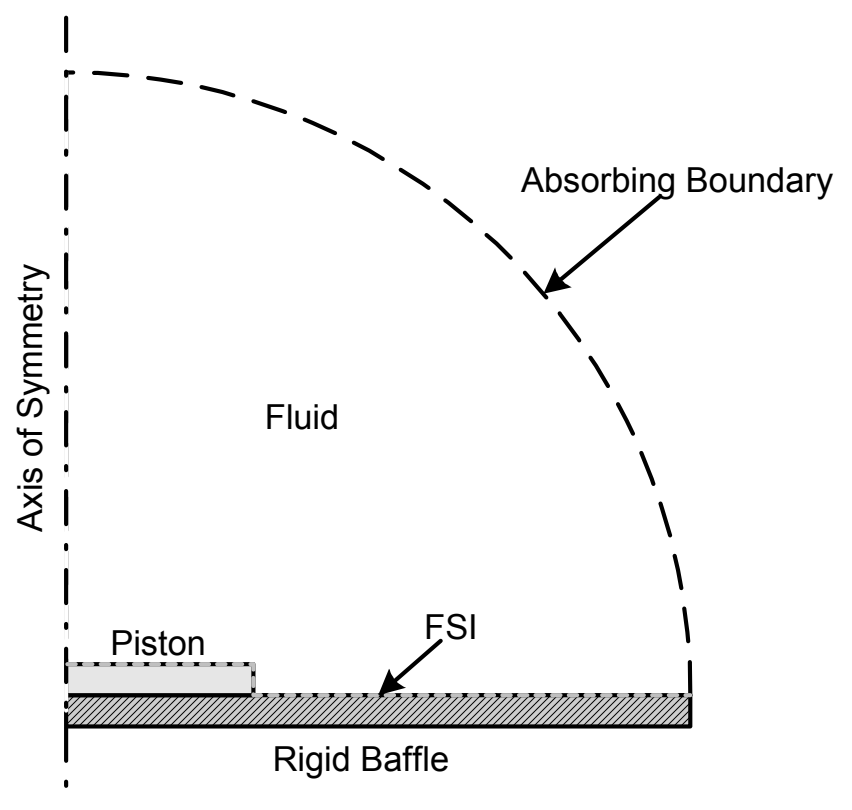

Figure 29 Illustration of the model of a vibrating piston on a baffle.

The fluid in the simulation is assumed to be linear and lossless. The elements used for the fluid, Fluid29, have a single degree of freedom, pressure, and are linear elements with only corner nodes. Vibrations of the solid are coupled to the fluid through the FSI region which forces the normal stress to be continuous across the interface. This results in twoway coupling from the solid to the fluid and vice-versa. For the FSI to work properly, the elements used in the mesh of the solid must also be linear with corner nodes only. For this reason, Solid42 was used for all of the solid regions.

The absorbing boundary condition is the last key element for the acoustic analysis. Since a harmonic analysis is carried out for the acoustic analysis, an absorbing boundary condition is needed to prevent waves, propagating away from the solid, from reflecting off of the edge of the model and generating an unrealistic standing wave field. This allows one to simulate an infinite fluid domain with a finite number of elements. 
The absorbing boundary condition requires that the boundary of the fluid region be a segment of a circular arc.

In the model of the baffled piston, the baffle is assumed to be rigid: all of the displacements are set equal to zero. The face of the piston is forced to have a ydisplacement at the harmonic excitation frequency. The model was then solved over a range of frequencies so that the radiation impedance could be calculated and compared with theory. For a baffled piston, the radiation impedance can be calculated analytically as [38]

$$
Z_{P}=\rho_{0} c_{0}\left[R_{1}(2 k a)+j X_{1}(2 k a)\right]
$$

where

$$
R_{1}=1-\frac{2 J_{1}(2 k a)}{2 k a}
$$

and

$$
X_{1}=\frac{2 K_{1}(2 k a)}{(2 k a)}
$$

In these equations, $k$ is the wave number $(\omega / c), a$ is the radius of the piston, and $\rho_{0}$ and $c_{0}$ are the density and sound speed in the fluid. The function $J_{l}(x)$ is the Bessel function of the first kind of order one; $K_{l}(x)$ is the Struve function of order one [40].

For the finite element model, the displacement and force data along the face of the piston at each frequency was used to calculate the radiation impedance from:

$$
Z_{F E M}=\frac{1}{\mathrm{v}_{\text {avg }}} \int_{A} p_{\text {Face }} \cdot d A
$$

where $\mathrm{v}_{\text {avg }}$ is the average velocity over the face of the piston and $p_{F a c e}$ is the pressure on the face of the piston. 
The analytical and finite element results for the radiation impedance of a baffled piston are plotted in Figure 30. The dashed line represents the analytical solution, while the solid line represents the FEM results.

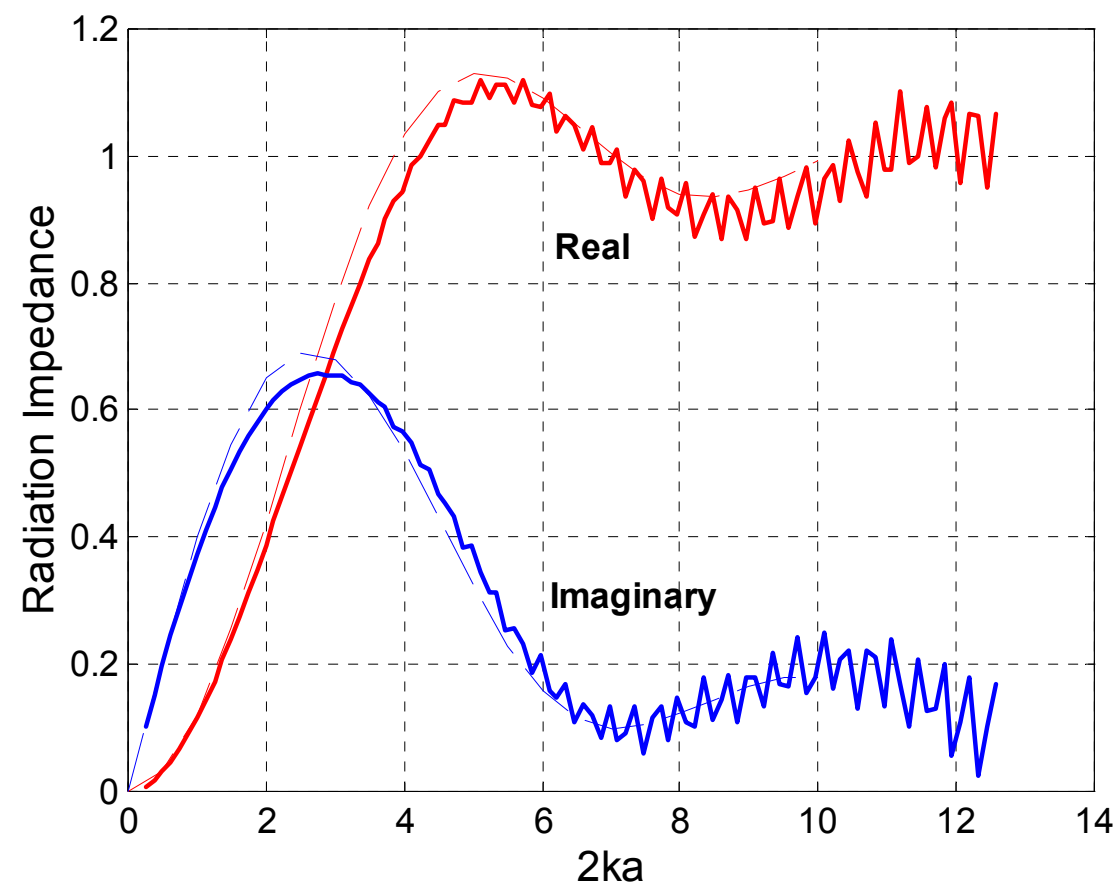

Figure 30 Plot of the real and imaginary parts of the radiation impedance of a baffled piston

From Figure 30, one can see that the finite element results match well with the analytical solution up to a $2 \mathrm{ka}$ value of 5 . After this value, the results from the finite element model begin to show signs of an insufficient mesh density. As the frequency is increased, the wavelength of sound in the fluid is reduced according to:

$$
\lambda_{\text {fluid }}=\frac{c_{\text {fluid }}}{f}
$$

where $\lambda_{\text {fluid }}$ is the wavelength of the acoustic wave in the fluid, $c_{\text {fluid }}$ is the speed of sound, and $f$ is the frequency of excitation. For a piston with a radius of $15 \mu \mathrm{m}$, a value of $2 \mathrm{ka}$ of 
5 corresponds to a frequency of nearly $40 \mathrm{MHz}$. For this frequency, the resulting mesh density in the model was approximately 15-20 elements/wavelength. From Figure 30, one can see that this represents the minimum mesh density required to obtain accurate results in the acoustic analysis. After this critical value, the model begins to break down due to insufficient mesh density.

The results of this analysis confirmed that the fluid structure interaction and absorbing boundary condition worked properly. The results also established that the minimum mesh density for the fluid region of the acoustic model must be at least 15 elements per wavelength at the frequency of interest.

\section{Infinitely Long Free Plate}

To investigate the waves propagating in the silicon plate, it was necessary to simulate an infinitely long plate using a finite number of elements. If the length of the plate were finite, a standing wave field would be generated in the plate which would mask the propagating modes. For the fluid region, an infinite fluid domain was simulated using an absorbing boundary condition built into ANSYS. However, there is no such condition for the solid region of the model. Therefore, the approach of Bozkurt et al. was used to simulate an infinitely long silicon plate [41]. Attenuating silicon regions at both ends of the plate were used to slowly attenuate the Lamb waves before they could reflect off of the ends of the model and create standing waves. The arrangement of the ideal and attenuating, or lossy, silicon regions is shown schematically in Figure 31. 


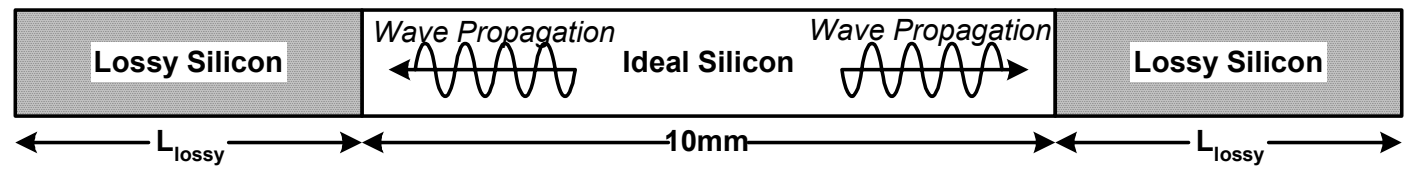

Figure 31 Illustration of the arrangement of attenuating regions of the silicon plate.

The lossy silicon was implemented by setting the stiffness matrix multiplier, BETAD, of the Rayleigh damping equation to a small value. The value of the damping coefficient, the length of the lossy region, and the change in mesh density were all found to have an impact on the amplitude of the reflections in the ideal silicon region.

A parametric study was performed to determine the optimum values of the aforementioned quantities for a $200 \mu \mathrm{m}$ silicon plate. The plate was excited by point forces on the top and bottom surfaces at the center of the plate at the desired frequency of $10 \mathrm{MHz}$ to generate $\mathrm{A}_{0}$ and $\mathrm{S}_{0}$ Lamb waves, as shown in Figure 32.

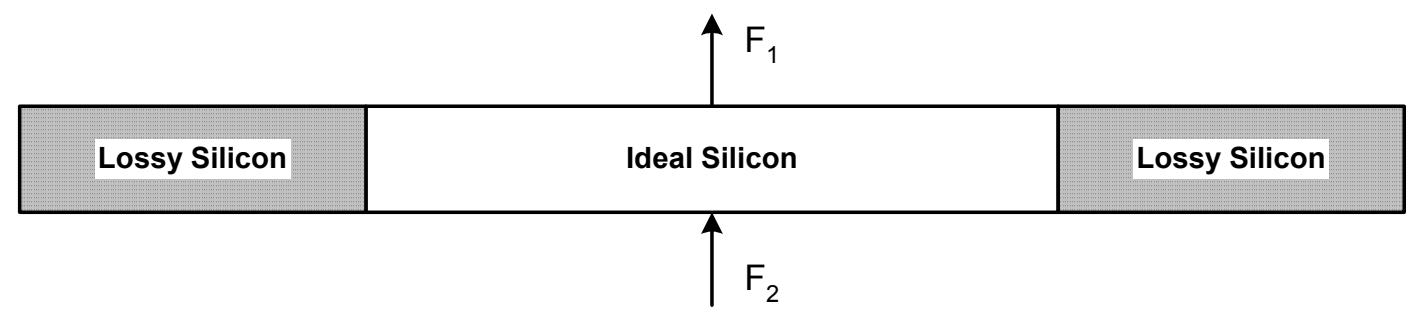

Figure 32 Illustration of method used to excite Lamb waves in the silicon plate.

To excite $\mathrm{A}_{0}$ Lamb waves, $F_{1}$ and $F_{2}$ had equal amplitudes and the same phase. To generate $\mathrm{S}_{0}$ Lamb waves, $F_{1}$ and $F_{2}$ had equal amplitudes but were $180^{\circ}$ out of phase.

The magnitude of the normal surface displacement along the length of the plate was used to judge the performance of the lossy region. A typical plot of the displacement 
amplitude is shown in Figure 33 to illustrate the presence of standing waves in the ideal silicon region.

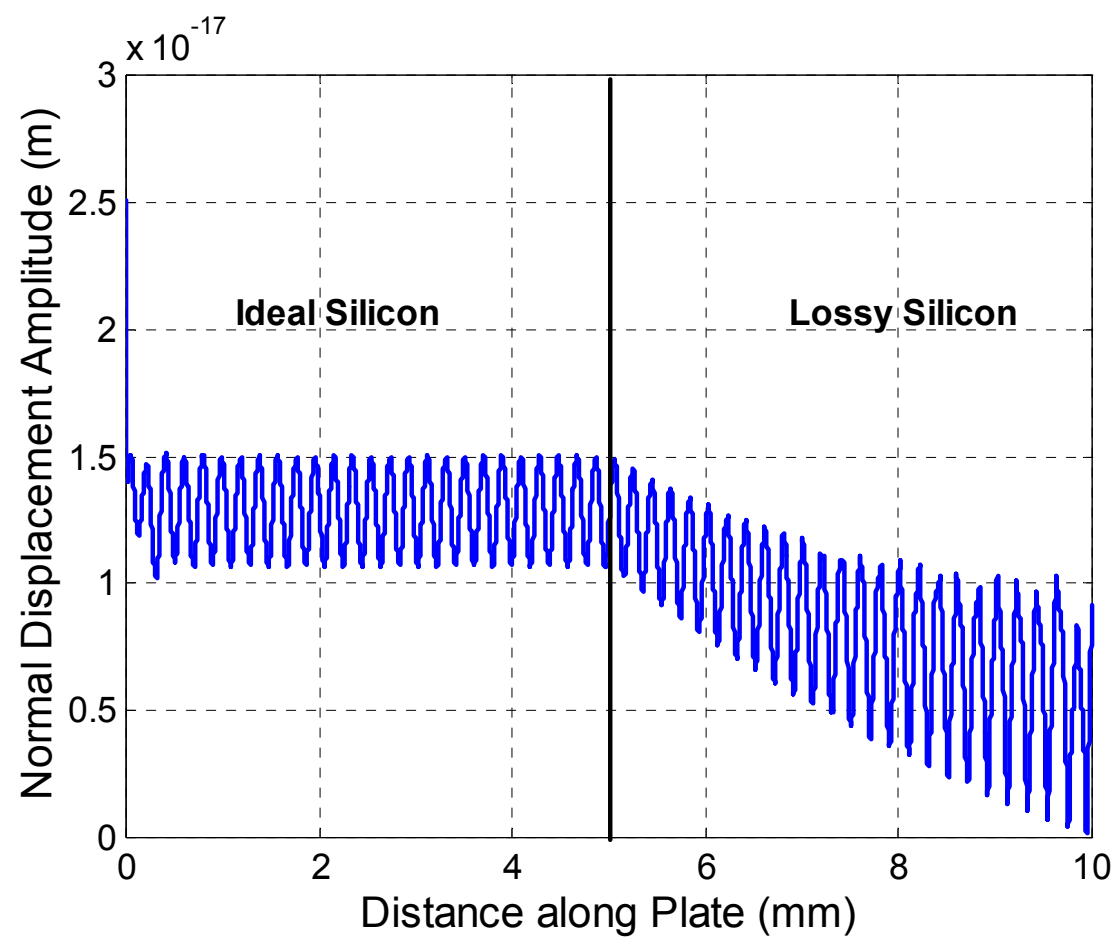

Figure 33 Plot of the amplitude of the normal surface displacement over the length of the silicon plate. The damping coefficient for this case was $5 \times 10^{-10}$.

If there were no reflections, the magnitude of the normal surface displacement would remain constant through the ideal region of the silicon and then decay in the lossy region. In Figure 33, one can see that there is a large variation in the displacement magnitude along the length of the plate. This is due to a standing wave created in the ideal region of the plate by reflected Lamb waves.

For waves propagating in a $200 \mu \mathrm{m}$ thick silicon plate excited at $10 \mathrm{MHz}$, the optimal values of lossy region length, damping coefficient, and rate of increase in mesh density were determined and are listed in Table 3. 
Table 3 Optimal values of parameters used to form the lossy silicon region.

\begin{tabular}{|c|c|}
\hline Parameter & Value \\
\hline Length of Lossy Region & $5 \mathrm{~mm}$ \\
\hline Damping Coefficient & $4 \times 10^{-9}$ \\
\hline Lossy Element Growth Rate & $60 \% / \mathrm{mm}$ \\
\hline
\end{tabular}

The third parameter in Table 3, the lossy element growth rate, refers to how quickly the size of the elements can be increased along the length of the lossy region. It is necessary to decrease the mesh density along the lossy region so that the maximum number of elements is not exceeded and so that the computation time can be reduced. The magnitude of the normal surface displacement for these optimal values is plotted in Figure 34 .

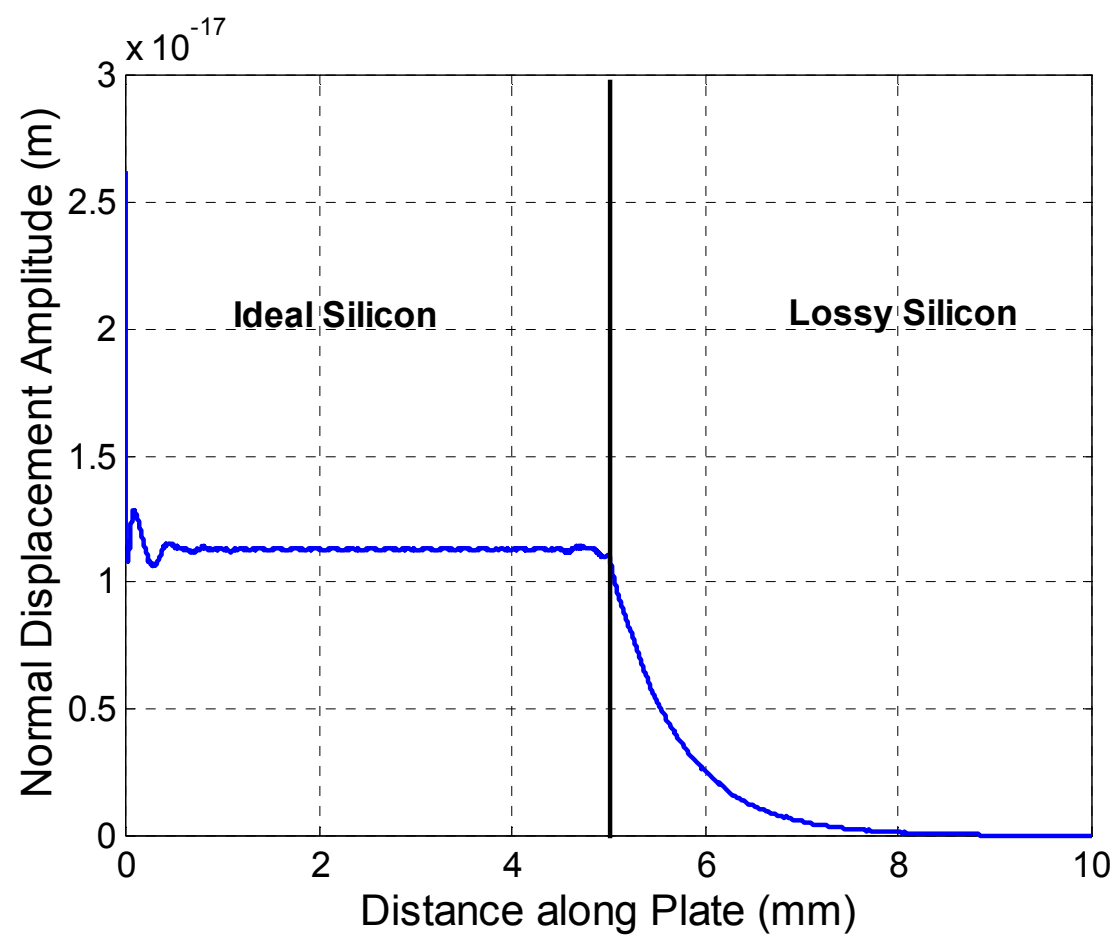

Figure 34 Plot of magnitude of normal surface displacement for optimal lossy silicon region. 
The variation in amplitude of the normal surface displacement for the optimal lossy silicon region was found to be less than $1.3 \%$, which is acceptable.

For simulations at other frequencies, the required length of the lossy regions was found to scale with the wavelength of the Lamb waves in the plate. For example, at low frequencies, the length of the lossy region must be longer to account for the fact that the wavelength is longer. The necessary mesh density was also found to scale with the wavelength of the modes, as expected.

\section{Fluid Loaded Plate}

The silicon plate with lossy silicon regions was coupled to a fluid half-space to verify that Lamb waves in the plate would radiate pressure waves into the surrounding fluid. Due to the large required length of the silicon plate, a total of $20 \mathrm{~mm}$, a semicircle of fluid could not be attached to the plate as for the baffled piston. This would require an impractically large number of elements. To avoid this problem, an approach was developed which places a circular arc of fluid on one side of the silicon substrate, drastically decreasing the required number of nodes. By employing the arc of fluid, one can model a larger substrate while maintaining a high mesh density in the fluid. The arc was formed by overlapping a large circle, with its center far below the substrate, with the silicon substrate. A schematic of the finite element model is shown in Figure 35.

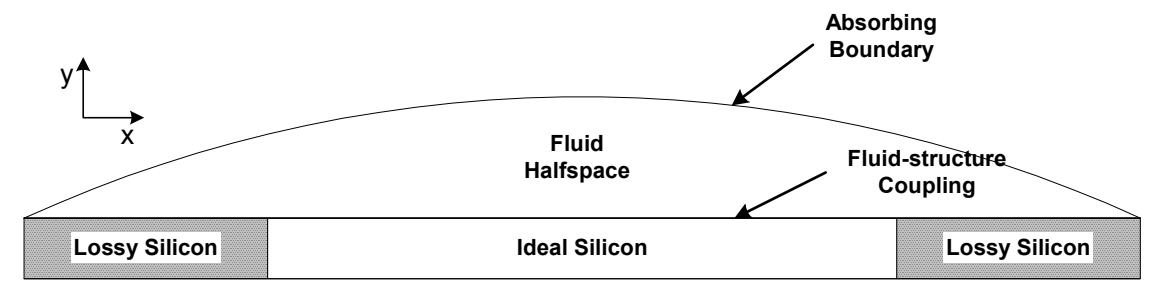

Figure 35 Illustration of arc of fluid coupled to the vibrating silicon plate. 
The plate was again excited with point forces at $10 \mathrm{MHz}$ to generate Lamb waves propagating to the left and right. The resulting pressure distribution in the fluid was calculated and is shown in Figure 36.

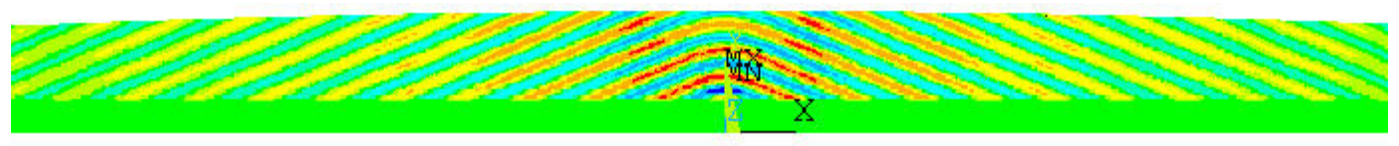

Figure 36 Plot of the pressure distribution in the fluid resulting from the propagation of $\mathrm{A}_{0}$ Lamb waves in the silicon plate.

In Figure 36, one can see that the Lamb waves generate plane waves radiating outward from the plate. The angle that the pressure waves make with respect to the normal of the plate, $\theta_{f}$, can be calculated from Snell's law,

$$
\theta_{f}=\sin ^{-1}\left(\frac{c_{f}}{c_{\text {trace }}}\right)
$$

where $c_{\text {trace }}$ is the phase velocity of the $\mathrm{A}_{0}$ Lamb wave and $c_{f}$ is the sound speed in the fluid. For a $200 \mu \mathrm{m}$ thick fluid loaded plate excited at $10 \mathrm{MHz}$, the analytical model predicts that the phase velocity of the $\mathrm{A}_{0}$ Lamb wave is $3884 \mathrm{~m} / \mathrm{s}$. For a fluid with a sound speed of $1500 \mathrm{~m} / \mathrm{s}$, Snell's law predicts that the Lamb wave will radiate a plane wave into the fluid which makes an angle of $22.7^{\circ}$ with the vertical. From the finite element results, the angle was found to be approximately $23^{\circ}$, which is an excellent match with the prediction.

The Fourier transform (FT) of the normal surface displacement was used to identify the modes propagating in the fluid loaded plate and their relative levels, following the approach of Certon et al.[42]. To identify the modes, the normal surface displacement was recorded along the length of the ideal silicon region. The FT of the 
complex displacement was computed in the computer and plotted against the phase velocity. The phase velocity was calculated from the following:

$$
V_{\text {phase }}=\left(\frac{1}{f_{F T}}\right) \cdot f_{\text {Excitation }}
$$

where $f_{F T}$ are the frequencies from the Fourier transform and $f_{\text {Excitation }}$ is the input excitation frequency $\left(10 \mathrm{MHz}\right.$ in this case). The values of $f_{F T}$ were calculated from:

$$
f_{F T}=\frac{(1: n)-1}{n \cdot d x}
$$

where $n$ is the number of points used in the Fourier transform and $d x$ is the spacing between data points on the silicon plate. The plot of the FT is plotted in Figure 37 for a fluid loaded plate with $\mathrm{A}_{0}$ excitation.

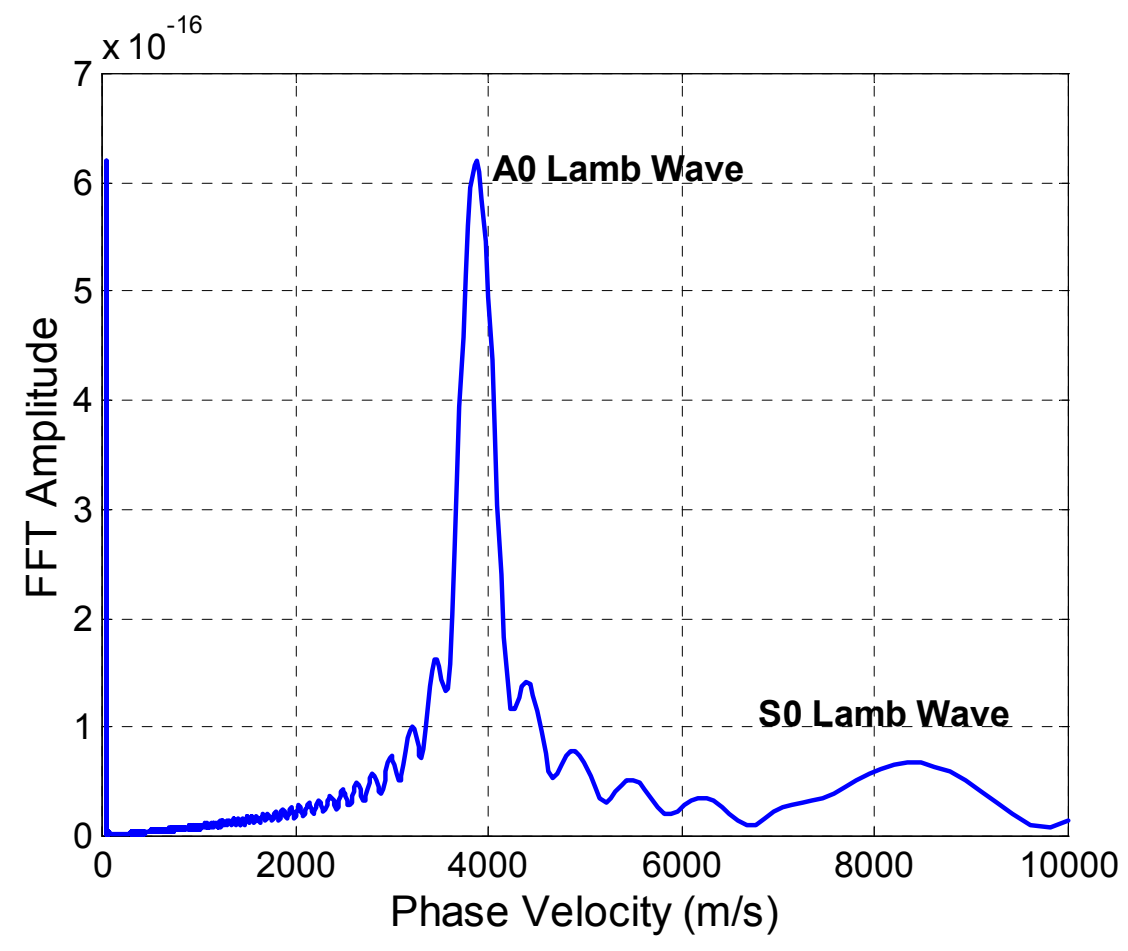

Figure 37 Fourier transform of normal surface displacement of silicon plate attached to water half-space and excited with point forces at $10 \mathrm{MHz}$. 
In the plot, one can see that there are two modes propagating in the silicon plate. The first mode propagates with a phase velocity of approximately $3880 \mathrm{~m} / \mathrm{s}$ which corresponds to the $A_{0}$ Lamb wave. The second mode propagates with a velocity of $8330 \mathrm{~m} / \mathrm{s}$ which corresponds to the $\mathrm{S}_{0}$ Lamb wave. The phase velocities of both modes match well with the results of the analytical model.

Next, a single vibrating membrane was placed on the surface of the fluid loaded plate to represent a single membrane of a cMUT array. The membrane was excited with a harmonically varying pressure at the desired frequency of $10 \mathrm{MHz}$. After solving the finite element model, the displacement data along the fluid-solid interface was recorded. The FT of the normal displacement data was then calculated and is plotted in Figure 38.

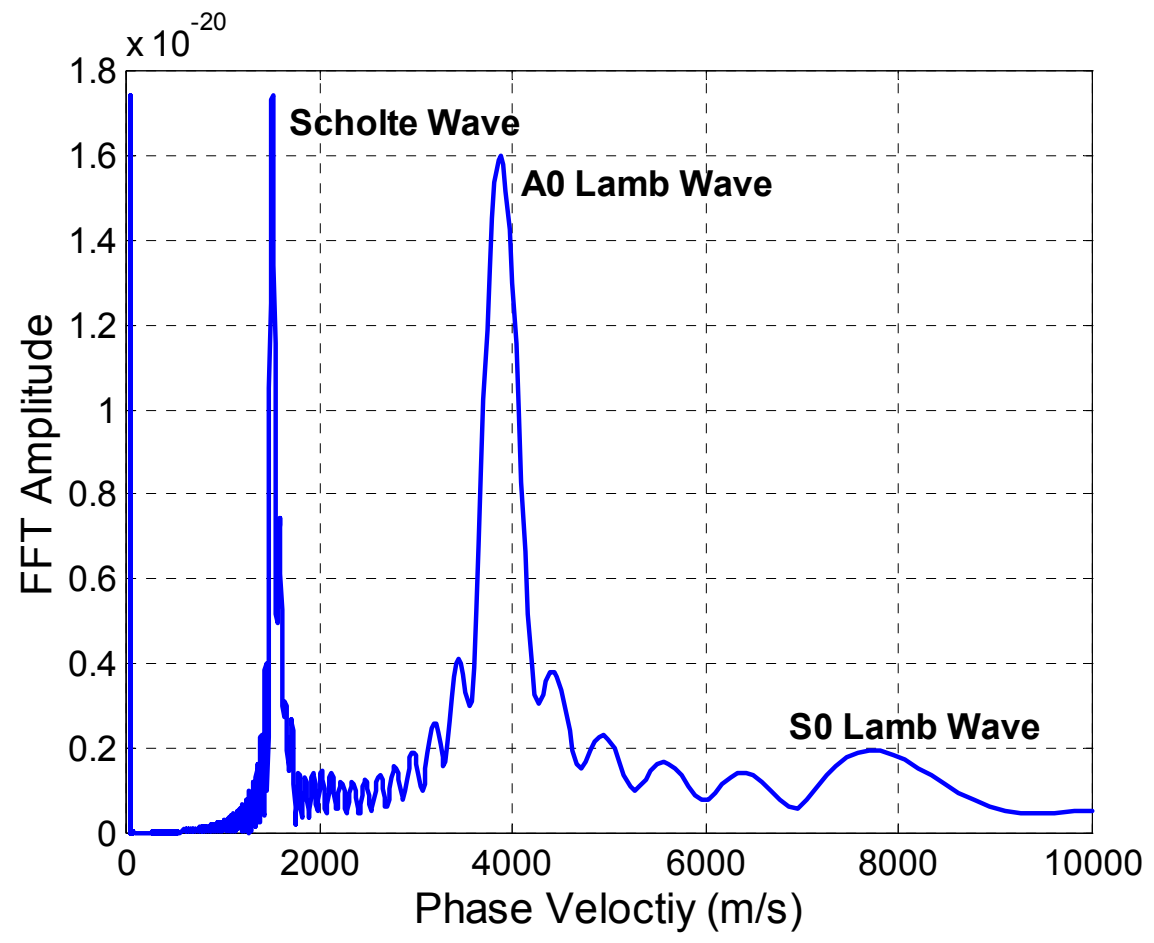

Figure 38 Fourier transform of normal surface displacement of a silicon plate with a single vibrating membrane attached to water half-space. 
The presence of the vibrating membrane causes an additional mode to be excited, namely the Scholte wave. Since the Scholte wave is an interface wave, it appears at a velocity much different that the Lamb wave velocities but very close to the fluid sound speed of $1500 \mathrm{~m} / \mathrm{s}$, as shown in Figure 38. Also note that the peaks for the Lamb waves are much broader as compared to the Scholte wave. This is expected since these modes leak their energy to the fluid and the resulting normal displacement exponentially decays in a short lateral distance. In contrast, the evanescent Scholte wave propagates along the whole length of the interface without attenuation and thus has a sharp peak.

\section{Simulation of Interdigital cMUT Array in Fluid Half-space}

After verifying the operation of the basic finite element model, an interdigital cMUT array was introduced into the model of a fluid half-space attached to a silicon plate, as shown in Figure 39.

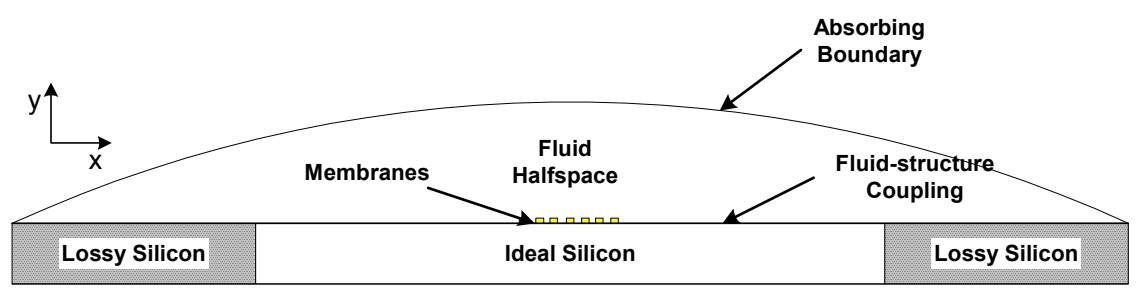

Figure 39 Illustration of finite element model for fluid half-space.

The membranes were excited by forces applied to the nodes so that complex loads could be applied to simulate the desired $90^{\circ}$ phase shift. All simulations were performed at a frequency of $10 \mathrm{MHz}$. A typical program used for simulating a cMUT array in a fluid half-space can be found in Appendix 2. 


\section{Model Validation}

A 5 finger pair, interdigital cMUT was simulated in a fluid half-space. The resulting pressure distributions in the fluid for the transducer operating at $10 \mathrm{MHz}$ on a $200 \mu \mathrm{m}$ silicon substrate are shown in Figure 40.

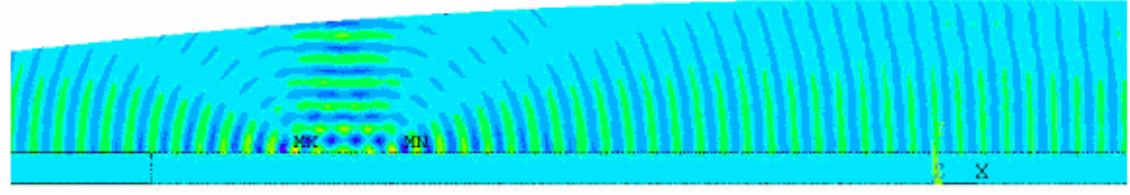

(a)

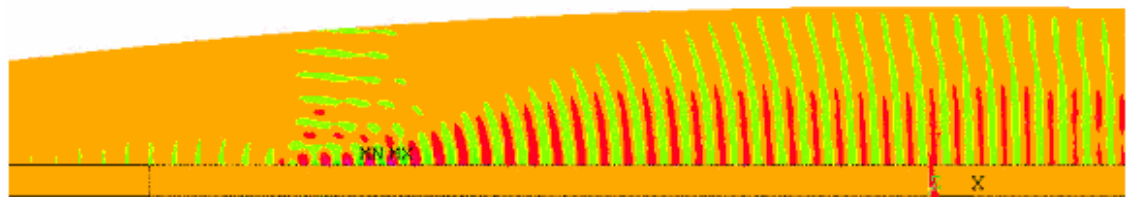

(b)

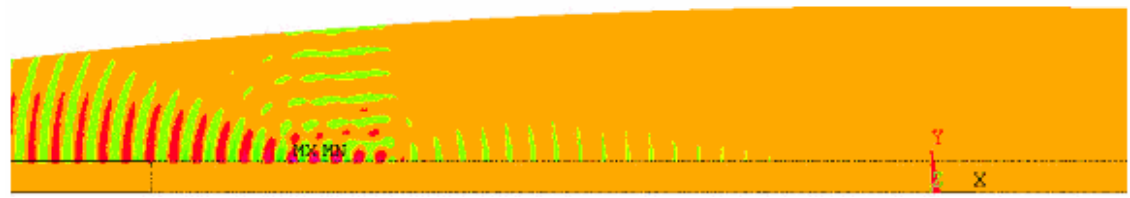

(c)

Figure 40 Finite element analysis result for pressure fields in water where the fingers of the interdigital cMUT shown in Fig. 1 are driven at $10 \mathrm{MHz}$ with signals: (a) in phase, (b) $-90^{\circ}$ out of phase, and (c) $+90^{\circ}$ out of phase. The total lateral distance in the shown area is $7 \mathrm{~mm}$.

The results show the ability to generate highly directional Scholte waves in the fluid, which appear as evanescent waves in the vertical direction while propagating in the lateral direction as planar wave fronts. By changing the phase of the inputs, one can generate a highly directional Scholte wave in either lateral direction. 
The results of the finite element analysis were used to determine the directionality and efficiency of the interdigital cMUTs in generating Scholte waves. The directionality of the transducer, $D$, is defined as the ratio of power in the Scholte wave propagating toward the receiver, $P_{\text {forward }}$, to the power in the Scholte wave propagating away from the receiver, $P_{\text {backward }}$ and is expressed in decibels,

$$
D=10 \cdot \log _{10}\left(\frac{P_{\text {forward }}}{P_{\text {backward }}}\right) .
$$

The acoustic efficiency, $\eta$, is defined as the ratio of the acoustic power in the forward propagating Scholte wave to the total acoustic power radiated into the fluid and is expressed as a percentage:

$$
\eta=\frac{P_{\text {Scholte, forvard }}}{P_{\text {total }}} \times 100 \% .
$$

For this calculation, the power in the Scholte mode was calculated at a location in the fluid far from the transducer. This was done so that the cylindrically spreading bulk waves in the fluid would have a small effect on the pressure field when compared to the Scholte wave. The pressure and pressure gradient data was recorded along a vertical line extending from the silicon substrate to the edge of the fluid region. The pressure gradient in the x-direction (parallel to the interface), $d p / d x$, was used to calculate particle velocity, $V_{x}$, in the fluid at the input frequency $\omega$ :

$$
V_{x}=\frac{1}{j \omega \rho} \frac{d p}{d x} .
$$

The intensity at each data point was calculated and then the intensity along the line was integrated to determine the power in the Scholte mode for a particular excitation scheme using the expression 


$$
P_{\text {Scholte }}=\frac{1}{2} \int_{0}^{\infty} \operatorname{Re}\left\{p \cdot V_{x}^{*}\right\} d y .
$$

The total acoustic power radiated into the fluid was calculated using the pressure and displacement information on the surface of the cMUT membranes. The calculations were very similar to those for the power in the Scholte mode except that the normal velocity of the surface of the membrane was calculated directly from the displacement of the membranes. Note that the preceding calculations neglect the power radiated into the Lamb waves. This is because for a cMUT immersed in a fluid the power levels in the Lamb wave modes are at least $15 \mathrm{~dB}$ below the power level in the Scholte mode according to calculations reported by McLean et al.[18].

As previously stated, the Fourier transform (FT) of the normal surface displacement of the silicon substrate can also be used to identify the propagating modes in the cMUT substrate and determine their relative levels [42]. This method can also be used as a more accurate method for determining the directionality of the Scholte wave. While the power method can also be used to determine the directionality, there is a portion of the Scholte wave energy in the substrate which is neglected in the calculations. The FT method takes into account all of the energy of the Scholte wave by looking at normal surface displacement of the silicon-water interface. The displacement information used in this analysis was taken along the fluid-solid interface over the lossless region of the silicon substrate. The amount of Scholte wave directionality achieved with a particular transducer was calculated by comparing the amplitude of the Scholte wave signal for the cases of forward ( $+90^{\circ}$ : transmit toward receiver) and backward $\left(-90^{\circ}\right.$ : transmit away from receiver) excitation, as shown in Figure 41. 


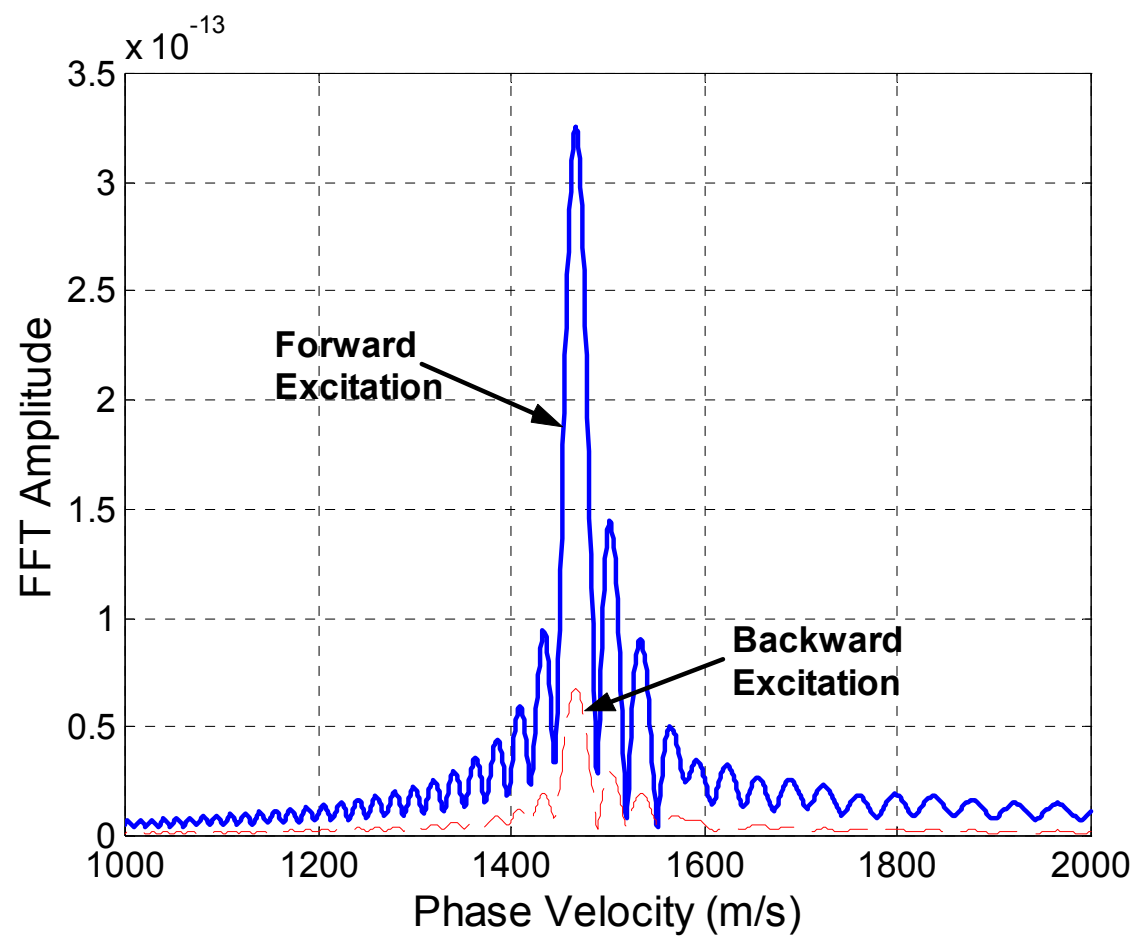

Figure 41 Fourier transform of normal surface displacement corresponding to forward and backward excitation of the Scholte wave.

These calculations show that a 5 finger pair transducer should produce a signal with 13.6dB of directionality. These results match well with the power based calculations which predict a directionality of $12.8 \mathrm{~dB}$ and justify the use of the FT technique for calculating directionality. The power based directionality is lower since there is a portion of the Scholte wave energy that is contained in the solid and is not taken into account in the calculation.

\section{Parametric Studies}

After establishing the evaluation metrics for analyzing interdigital cMUT arrays, parametric studies were performed to determine the effect of the number of fingers, the membrane periodicity, and relative phase on directionality and efficiency. All of the 
simulations were performed at $10 \mathrm{MHz}$. First, the number of finger pairs on the silicon substrate was gradually increased from 1 to 20 . The directionality as a function of the number of fingers is shown in Figure 42.

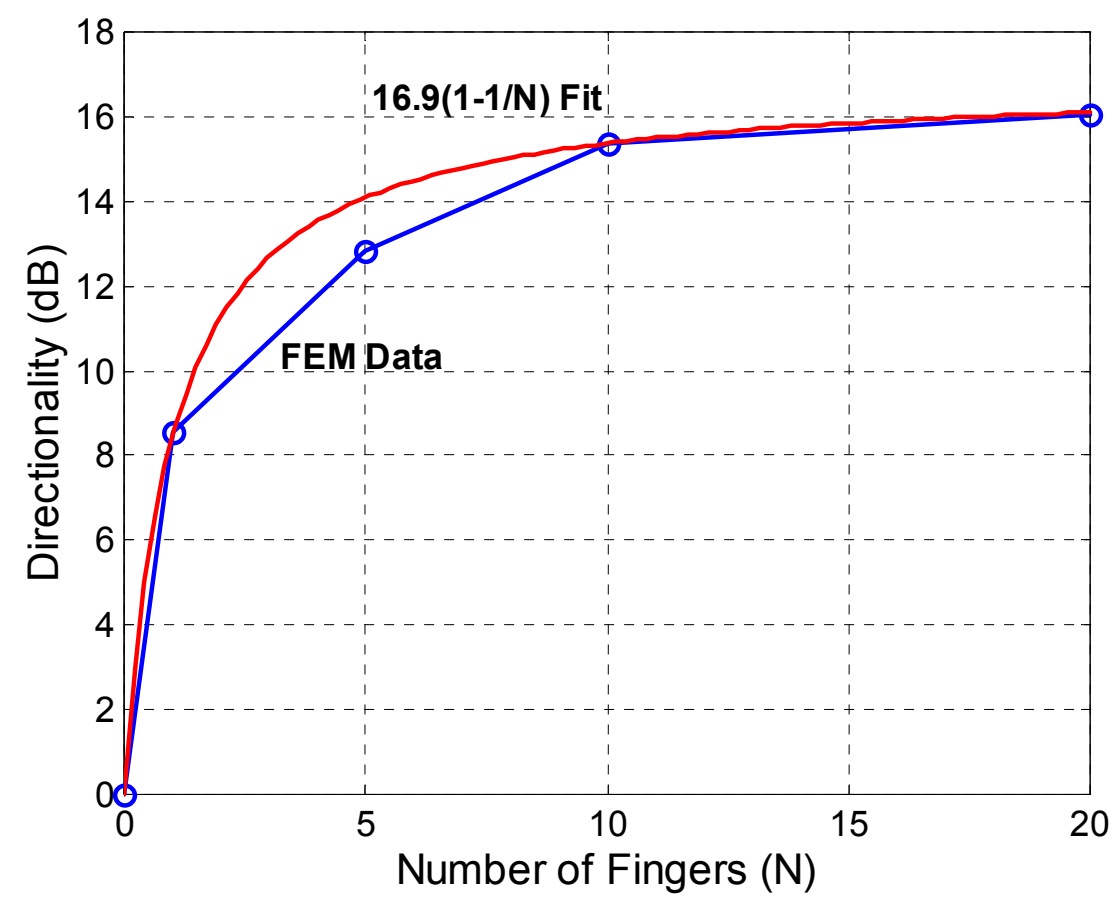

Figure 42 Directionality of an interdigital cMUT array as a function of the number of finger pairs in a fluid half space.

From the figure, one can see that the directionality increases with an increasing number of fingers. The equation for the curve that best fits the finite element results shown in Figure 42 is

$$
D=16.9\left(1-\frac{1}{N}\right)(d B)
$$

where $D$ is the directionality. The maximum directionality for this transducer is thus $16.9 \mathrm{~dB}$. The results show that the directionality cannot go to infinity, which is logical since there will always be some amount of energy propagating in the undesired direction. 
For a 20 finger pair device, the directionality was determined to be $16 \mathrm{~dB}$ which is $95 \%$ of the maximum value. Therefore, this is a suitable number of finger pairs for obtaining experimental results that can be compared with results for an infinite number of finger pairs. In practice, the maximum number of fingers in the transducer is dictated by the lateral dimensions of the silicon die. For pumping applications, a large number of fingers can be used, essentially covering the entire bottom of the channel. However, for sensing applications, there must be enough space between transmitter and receiver so input and output signals can be separated in time.

The efficiency in generating Scholte waves was also found to increase as the number of fingers was increased, as shown in Figure 43.

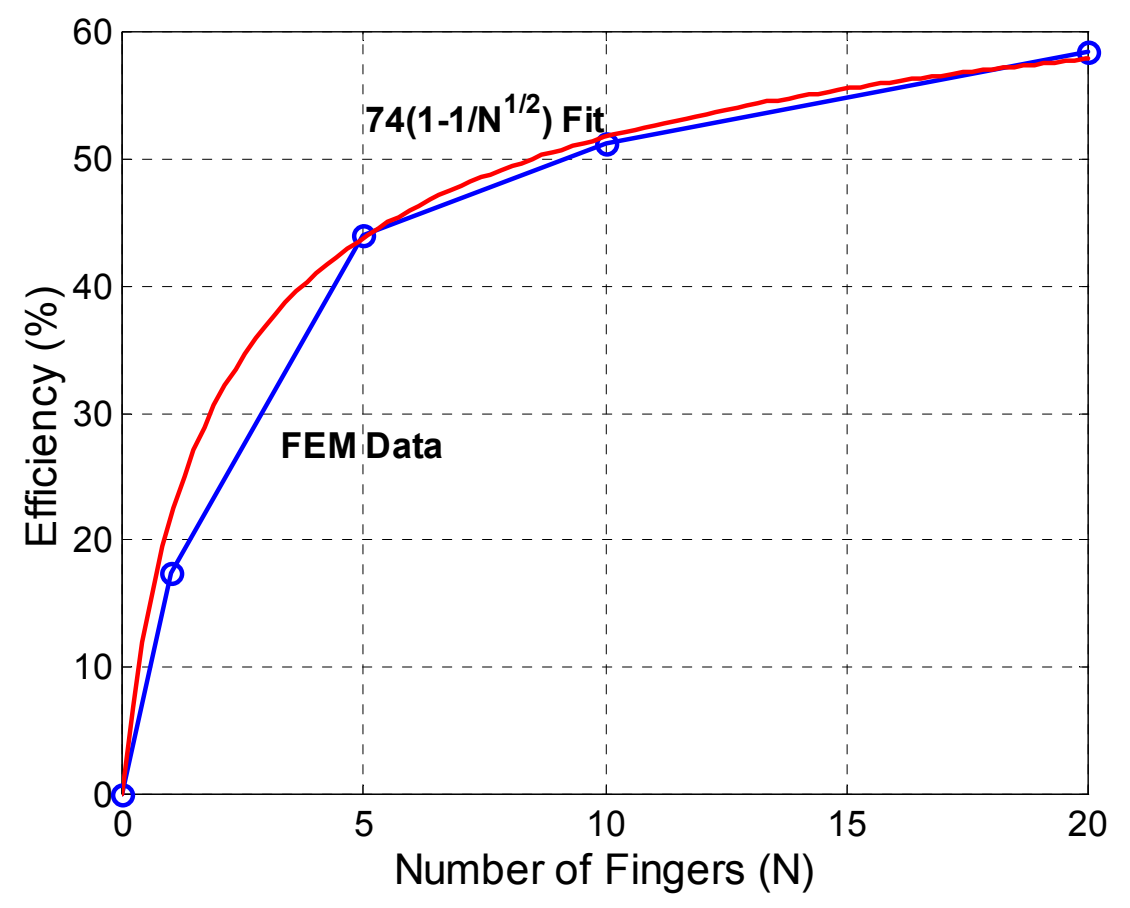

Figure 43 Efficiency in generating Scholte waves as a function of the number of fingers. 
The interdigital transducer structure acts as a filter which couples more energy into the mode of interest as the number of fingers is increased. In this case the mode of interest is the Scholte wave, and the efficiency has a value of nearly $60 \%$ for a 20 finger device. This efficiency refers to the amount of power in the forward propagating Scholte wave as compared to the amount of power radiated into the fluid by the membranes of the cMUT array.

The sound speed of the fluid was varied while the periodicity of the transducer was held constant to determine the optimum transducer pitch. The efficiency was calculated and is plotted as a function of the normalized wavelength in Figure 44.

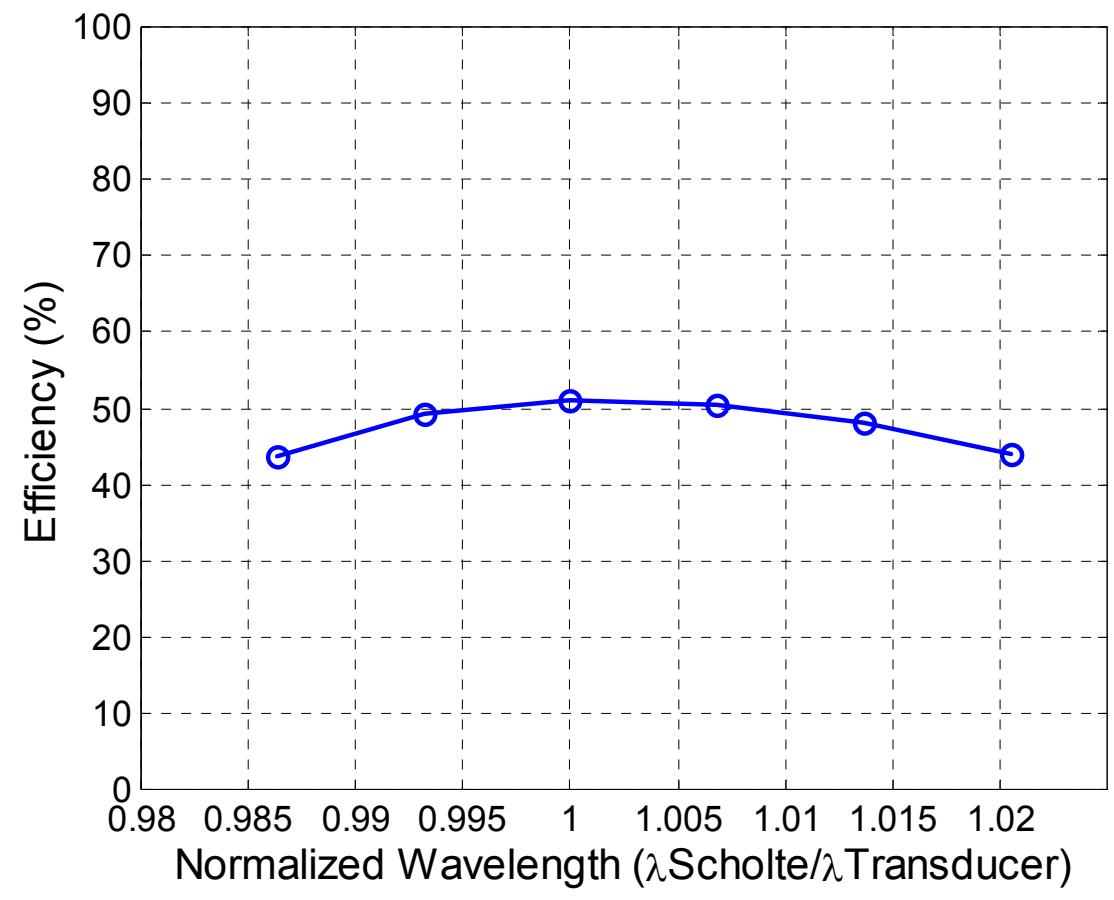

Figure 44 Efficiency as a function of the normalized wavelength for a 10 finger transducer.

From the figure, one can see that the efficiency reaches a maximum when the periodicity of the transducer matches the periodicity of the Scholte wave. For an interdigital cMUT 
operating in a water half-space at $10 \mathrm{MHz}$, the optimum finger periodicity is approximately $150 \mu \mathrm{m}$. For fluid sensing experiments, the finite element results can be used to determine the decrease in Scholte wave amplitude due to mismatch between $\lambda_{\text {Transducer }}$ and $\lambda_{\text {Scholte. }}$ The remaining decrease in received signal amplitude can be attributed to attenuation, and thus the viscosity of the fluid can be determined.

For a 10 finger device with optimum spacing, the effect of relative phasing on directionality and efficiency was investigated. The finite element model was solved for values of relative phase between the two legs of the transducer ranging from 0 to $360^{\circ}$. The directionality as a function of phase is plotted in Figure 45.

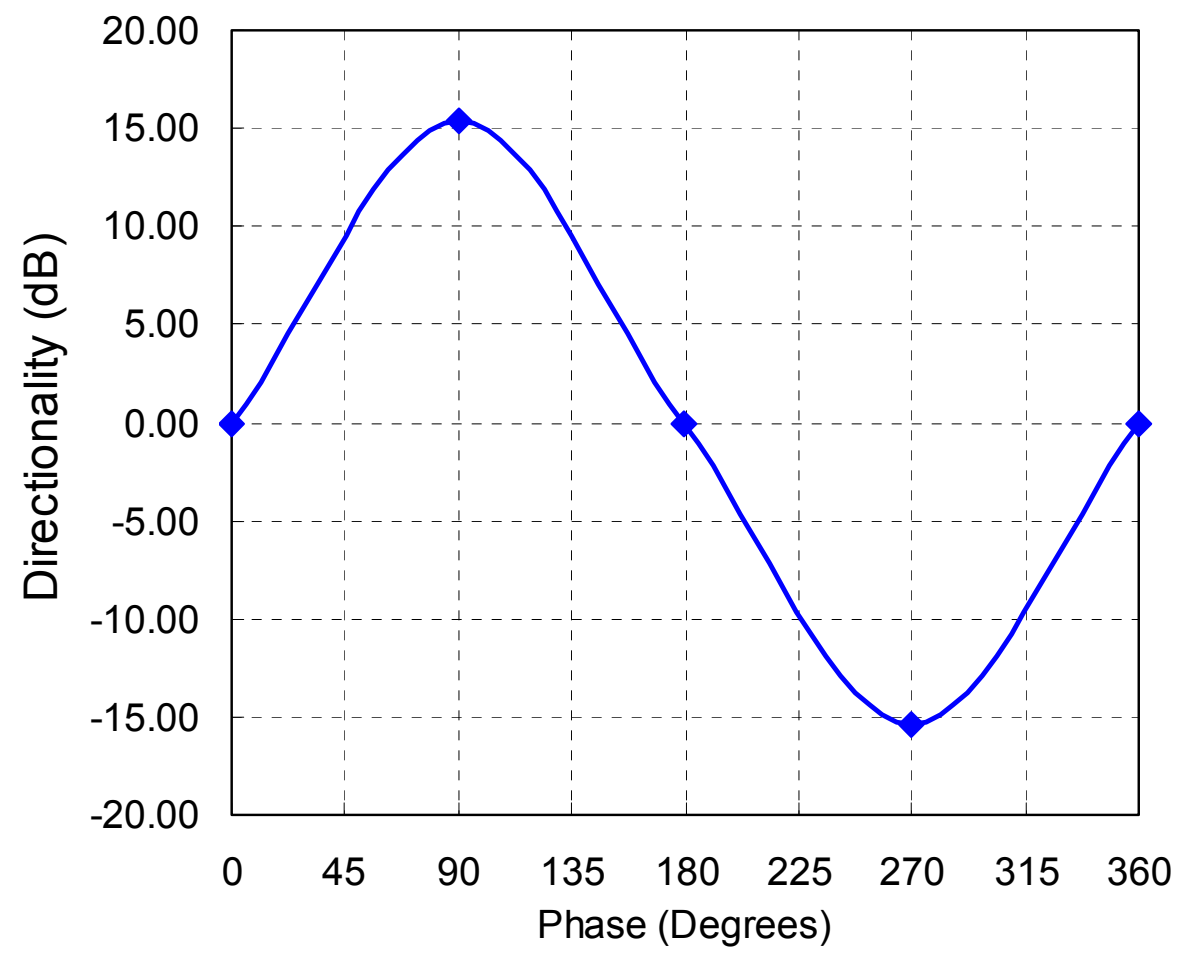

Figure 45 Directionality as a function of phase for a 10-finger interdigital cMUT.

In Figure 45, the maximum values for directionality occur at $\pm 90^{\circ}$ and have a magnitude of $15.3 \mathrm{~dB}$. When the directionality is positive, the Scholte wave propagates in the 
forward direction, and when the directionality is negative, the wave propagates in the backward direction. At $0^{\circ}, 180^{\circ}$, and $360^{\circ}$, the transducer has a directionality of $0 \mathrm{~dB}$ which implies that a bi-directional Scholte wave is generated.

The amount of power in the Scholte wave was calculated and compared to the total acoustic power input by the vibrating membranes, and the results are plotted in Figure 46.

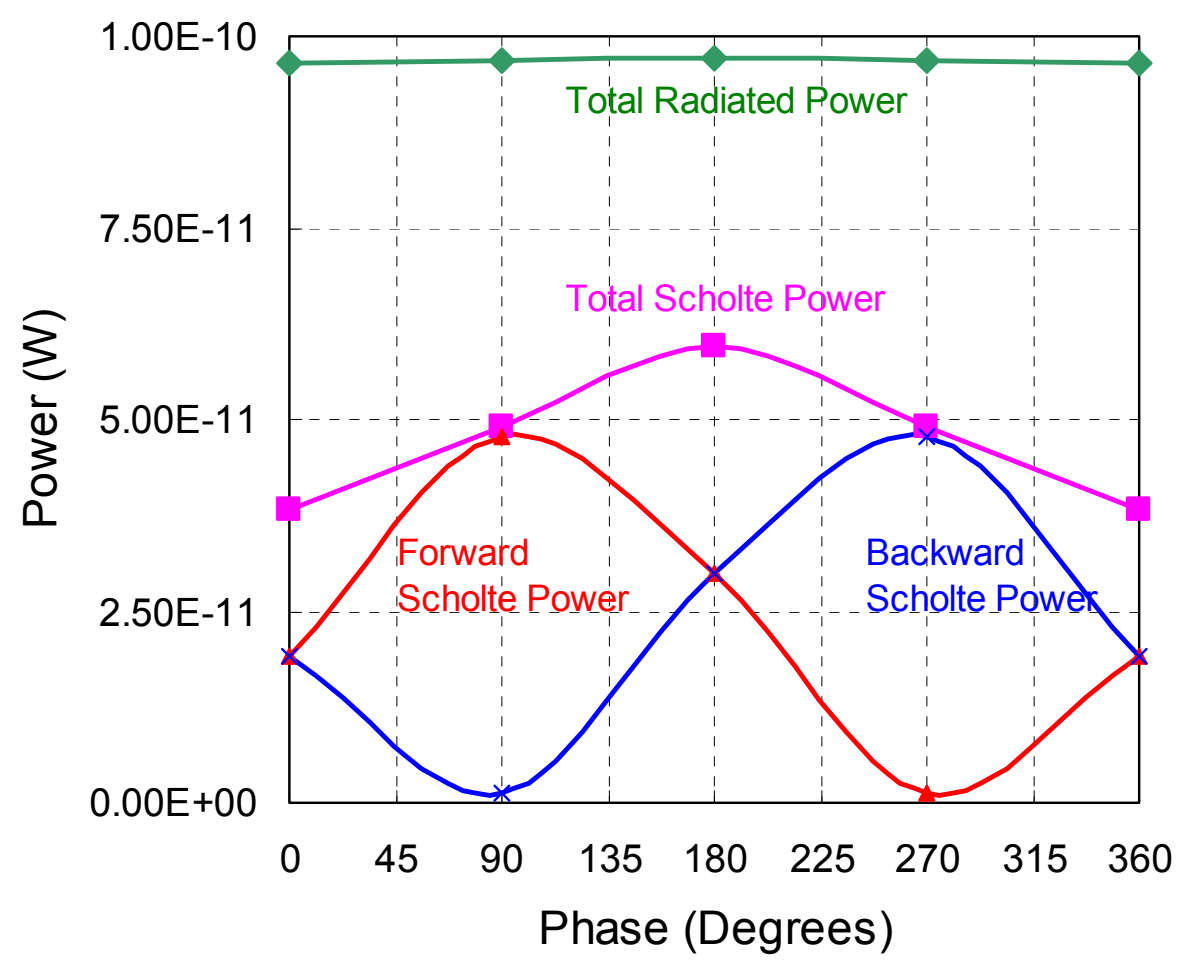

Figure 46 FEM results for an interdigital cMUT in a water half-space illustrating the total radiated power, total power in the Scholte wave, and directional Scholte wave power as a function of phase.

From the results shown in Figure 46, one can see that the total power radiated to the fluid remains relatively constant with phase. At $0^{\circ}$ and $360^{\circ}$, the smallest amount of power is coupled to the Scholte wave since the transducer acts as a more efficient bulk wave transducer. Also, the same amount of power goes into the forward and backward 
propagating Scholte waves when the phase is $0^{\circ}$ and $360^{\circ}$, as expected. The largest amount of power is coupled to the Scholte wave when the relative phase between the legs of the transducer is $180^{\circ}$. At this point $62 \%$ of the total power radiated by the membranes goes into the Scholte wave. However, the same amount of power is coupled to the forward and backward propagating Scholte waves, making the efficiency in generating a Scholte wave in a particular lateral direction only $32 \%$. When the relative phase is $90^{\circ}$, $49 \%$ of the total power radiated into the fluid goes into the forward propagating Scholte wave. This represents a significant improvement in coupling power to a Scholte wave in a single lateral direction. The results show that the relative phase between the legs of the transducer should be $90^{\circ}$ to couple the most power to the Scholte wave propagating in a single lateral direction.

\section{Interdigital cMUT Array in Fluid-Filled Microchannel}

CMUTs were also simulated in two-dimensional fluid channels to investigate directionality, efficiency and the generation of guided modes. The model is illustrated in Figure 47 and makes use of the same basic elements as the half-space model, only in a different arrangement.

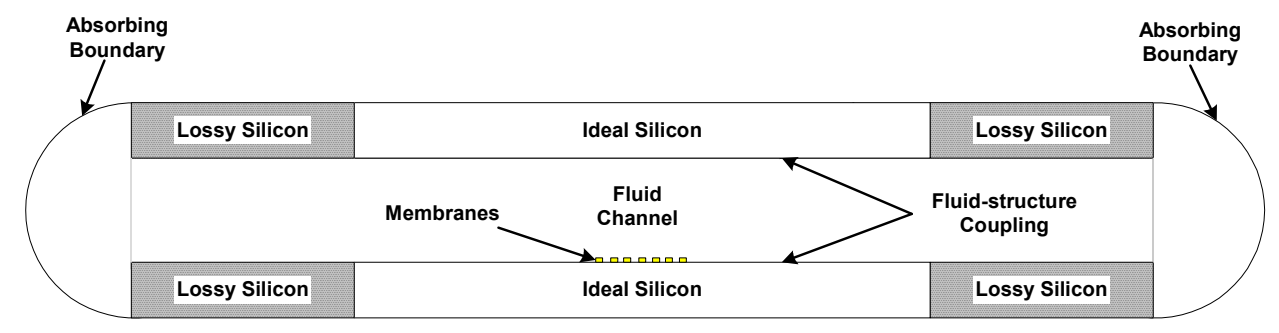

Figure 47 Illustration of finite element model for fluid filled microchannel. 
The combination of the absorbing boundary conditions on the ends of the fluid region and the attenuating silicon allows one to simulate a fluid channel of infinite length.

\section{Guided Mode Generation}

An interdigital cMUT designed for operation at $10 \mathrm{MHz}$ was simulated in fluidfilled channels of various heights to verify that guided modes could be generated in this structure. The pressure distributions for a 5 finger pair transducer in two different channels are shown in Figure 48.

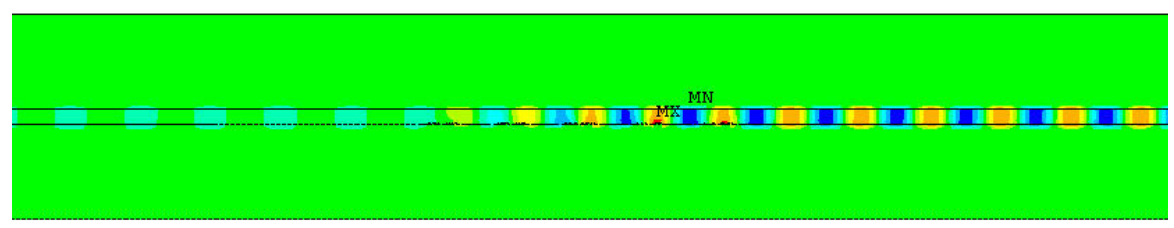

(a)

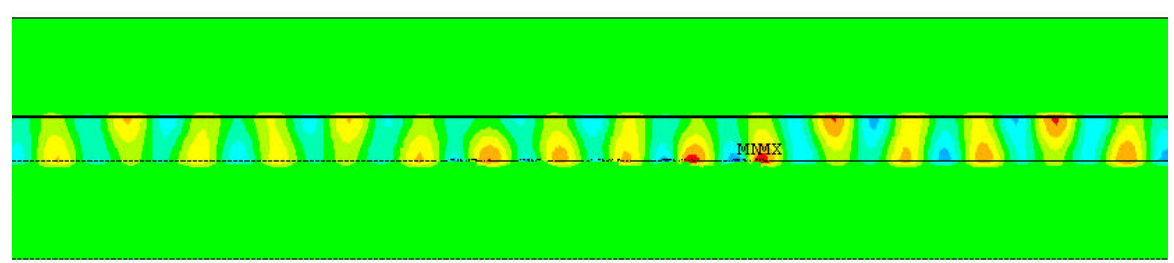

(b)

Figure 48 Pressure distribution in (a) $30 \mu \mathrm{m}$ and (b) $90 \mu \mathrm{m}$ channels resulting from forward excitation of 5 finger pair Scholte wave transducer at $10 \mathrm{MHz}$.

In Figure 48(a), the channel height is $30 \mu \mathrm{m}$ and only a single mode can propagate: the Scholte wave. The presences of the Scholte wave is suggested by the planar wavefronts in the pressure distribution and is confirmed by the FFT of the normal surface displacement. In Figure 48(b), the channel height is increased to $90 \mu \mathrm{m}$ which allows the 
Scholte wave and the first guided channel mode to propagate. The guided wave is indicated by the non-planar wavefronts in the pressure distribution.

The results are important because they show the ability to generate directional waves in fluid channels of various heights and indicate a bidirectional pumping capability.

The Fourier transform of the normal surface displacement of the fluid-solid interface was used to determine the velocity of the modes propagating in the fluidchannel structure. The FT results are plotted in Figure 49 for a range of channel heights.

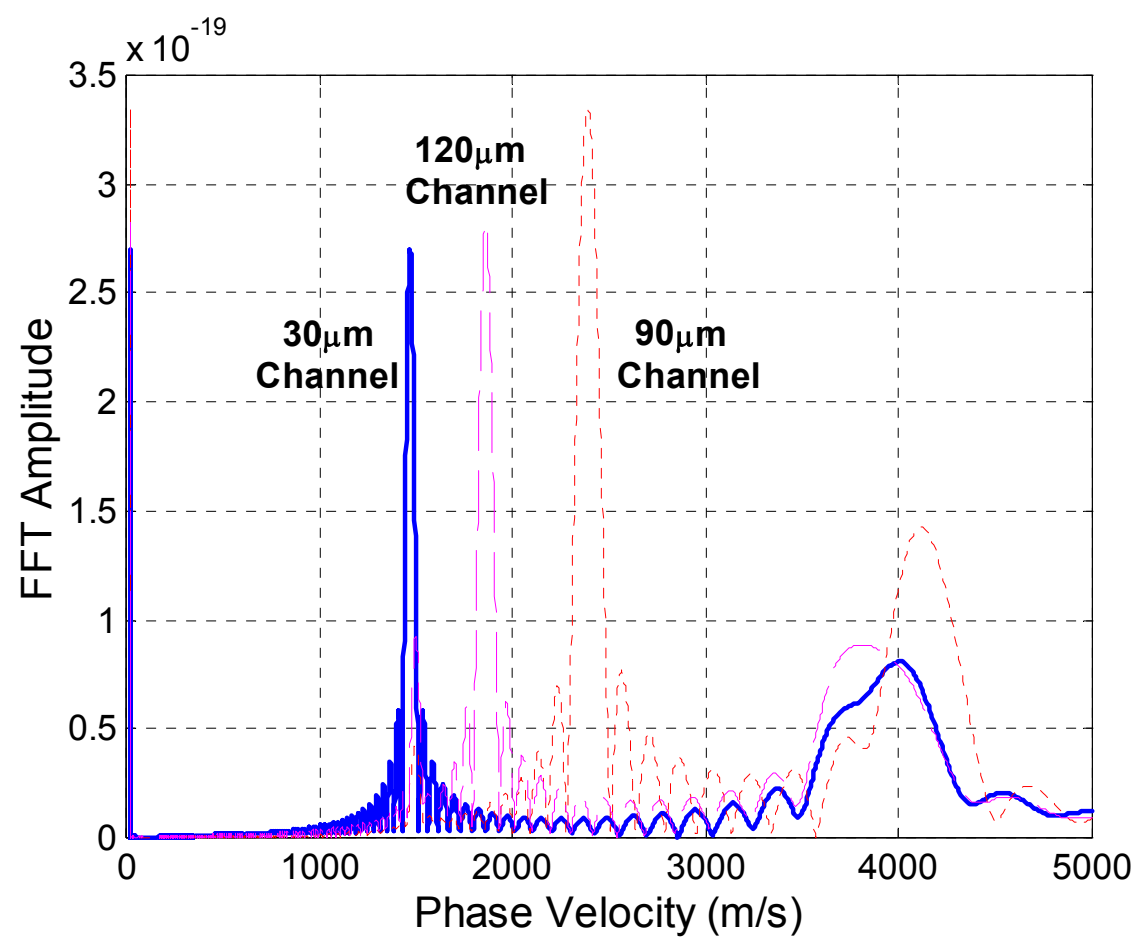

Figure 49 FT of normal surface displacement plotted against the phase velocity of the propagating modes for channel heights of 30,90 , and $120 \mu \mathrm{m}$.

For the $30 \mu \mathrm{m}$ channel, the only modes propagating are the Scholte wave and the symmetric and antisymmetric Lamb waves. When the channel height is increased to 90 $\mu \mathrm{m}$, an additional mode appears which propagates with a phase velocity of $2385 \mathrm{~m} / \mathrm{s}$. The velocity of this guided mode decreases to $1860 \mathrm{~m} / \mathrm{s}$ when the channel height is further 
increased to $120 \mu \mathrm{m}$. This mode is the first guided channel mode and the dispersion matches well with the analytical results (Figure 25).

\section{$\underline{\text { Parametric Studies }}$}

The effect of the relative phase between the membranes of a finger pair was investigated for an interdigital cMUT in a fluid-filled microchannel. The channel was filled with $100 \mu \mathrm{m}$ of water and was excited at $10 \mathrm{MHz}$. The periodicity of the fingers was set equal to the Scholte mode wavelength for the fluid-properties, frequency, and geometry of interest. The power coupled to the guided modes was calculated and compared to the total power radiated by the membranes, and the results are shown in Figure 50. 


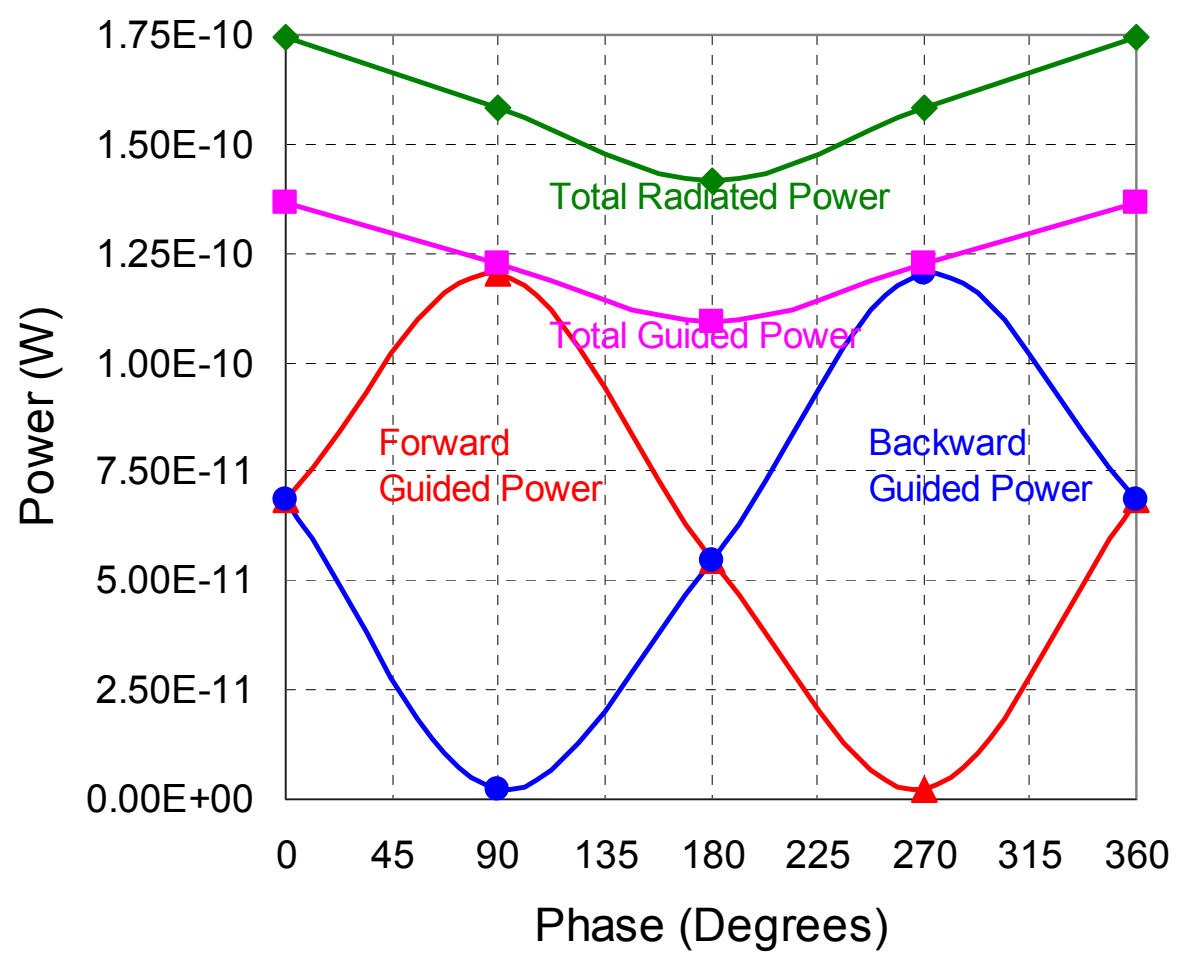

Figure 50 FEM results for an interdigital cMUT in a $100 \mu \mathrm{m}$, water-filled channel illustrating the total radiated power, total power in the guided waves, and the directional guided wave power as a function of phase.

The total power radiated by the transducer was calculated by integrating the intensity across the membranes. The power in the guided modes was calculated by integrating the intensity in the fluid at a cross-section of the channel far from the transducer. In Figure 50 , one immediately notices that the majority of the power radiated by the membranes goes into the guided waves. This is due to the fact that the fluid channel structure has a finite thickness, unlike the fluid half-space. Therefore, the power radiated by the membranes can only propagate in the lateral directions. The maximum power coupled to the guided waves in a preferred lateral direction occurs at angles of $90^{\circ}$ and $270^{\circ}$, when the directionality is at a maximum. For these two phase angles, approximately $80 \%$ of the 
total acoustic power goes into guided waves propagating in a single lateral direction. This is very attractive for fluid sensing and actuation applications. The maximum directionality resulting from this 10 -finger interdigital cMUT was found to be $17.5 \mathrm{~dB}$.

In conclusion, the efficiency in generating a guided mode in a preferred lateral direction improves as directionality is increased. Thus, the most energy is coupled into the Scholte wave in a single lateral direction when the phase, $\phi$, is given by

$$
\phi=90^{\circ} \pm m \cdot 180^{\circ}, \quad m=0,1,2,3, \ldots .
$$

The maximum power is delivered to the fluid when the relative phase between the membranes of a single finger is $180^{\circ}$. However, this point has a directionality of $0 \mathrm{~dB}$ and is thus unsuitable for pumping. 


\section{CHAPTER 5}

\section{LOW TEMPERATURE CMUT FABRICATION}

Based on the results of the analytical and finite element models, cMUTs were designed to operate at three frequencies, 5,10 and $20 \mathrm{MHz}$, to determine the effects of frequency and attenuation on fluid sensing and actuation performance. The frequency of $5 \mathrm{MHz}$ was selected as the low end of the range since the Scholte wave is essentially nondispersive for frequencies greater than or equal to $5 \mathrm{MHz}$. The upper frequency limit of $20 \mathrm{MHz}$ was determined by the practical limits of the fabrication process.

Transducers were fabricated for two distinct applications: sensing and actuation. CMUTs designed for sensing have two identical arrays separated by a prescribed distance on the silicon substrate, as shown in Figure 51(a). The lateral distance was designed so that the output signal from the receiver could be separated in time from the electromagnetic feed-through of the input signal. For example, a 10 cycle tone burst at $5 \mathrm{MHz}$ has a duration, $\Delta t$, of $2 \mu \mathrm{s}$. To ensure that the feed-through did not interfere with the received signal, the transducers were designed to have a propagation delay of at least $2 \Delta t$, or $4 \mu \mathrm{s}$. This corresponds to a distance of $8.67 \mathrm{~mm}$ between the transmitter and receiver, which established the minimum lateral dimensions of the transducer. Devices designed for operation at 10 and $20 \mathrm{MHz}$ had similar separations between transmitter and receiver. A scaled drawing of a sensing device designed for operation at $10 \mathrm{MHz}$ is shown in Figure 51. For each frequency, transducers were designed with different numbers of finger pairs, 5 and 10, so that the finite element models could be validated. 


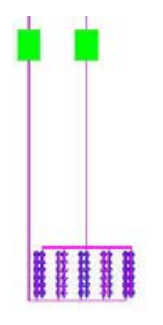

Transmitter
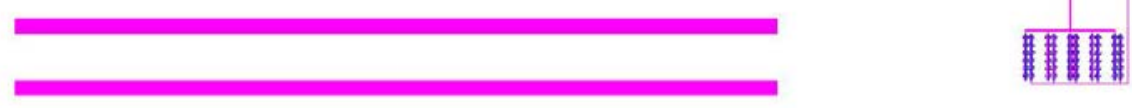

Receiver

(a)

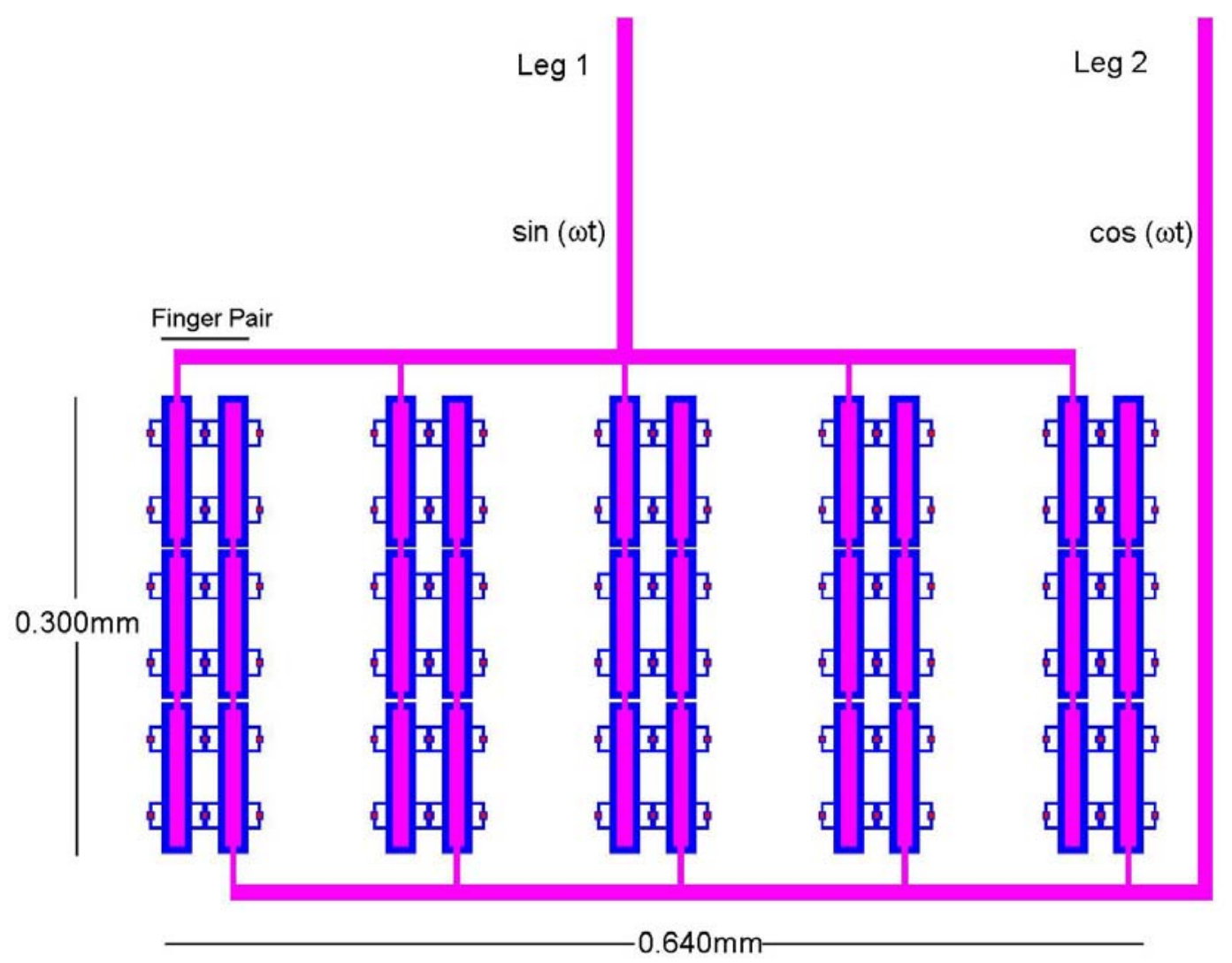

(b)

Figure 51 (a) Scaled drawing of sensing devices designed for operation at 10MHz. The spacing between the transmitter and receiver is $8.26 \mathrm{~mm}$. (b) Close-up image of a single array which has overall dimensions of $0.64 \mathrm{~mm}$ by $0.300 \mathrm{~mm}$. 
As one can see in Figure 51, each finger pair is made up of two rows of membranes. These rows are excited with time harmonic signals that are $90^{\circ}$ out of phase: sine and cosine inputs. The rows of a single finger pair are separated by one quarter of the Scholte mode wavelength, in this case $36.5 \mu \mathrm{m}$. The next finger pair is located at a distance of $146 \mu \mathrm{m}$, which corresponds to a full wavelength of the Scholte mode. For the $10 \mathrm{MHz}$ devices, each individual membrane is $100 \mu \mathrm{m}$ long and $20 \mu \mathrm{m}$ wide.

CMUTs designed for actuation had a single interdigital array located in the center of the silicon die. These transducers have a greater number of finger pairs to generate highly directional Scholte waves and to couple more power to the fluid. A device designed for pumping at $10 \mathrm{MHz}$ is illustrated in Figure 52.
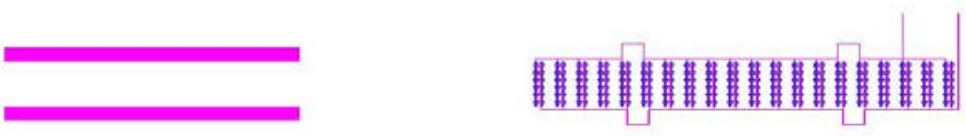

Figure 52 Scaled drawing of pumping device designed for operation at 10MHz. This transducer has 20 finger pairs to generate highly directional Scholte waves. 


\section{$\underline{\text { Review of Established cMUT Fabrication Processes }}$}

As previously described, a cMUT senses the arrival of an acoustic wave by measuring a change in the capacitance resulting from the vibration of the top electrode relative to the fixed bottom electrode. If the elements of the cMUT array have a small mechanically active area covered with an electrode, the change in capacitance will also be small, and can be overwhelmed by parasitic capacitance. A major source of parasitic capacitance comes from the electrical interconnects between the silicon die containing the transducer and the off-chip amplifying electronics. This source of parasitic capacitance can only be reduced through hybrid or monolithic integration with the electronics which would typically be implemented using complementary metal oxide semiconductor (CMOS) technology.

The original cMUT fabrication process developed at Stanford University is based on surface micromachining and makes use of low-pressure chemical vapor depositions (LPCVD) for forming the sacrificial layer and the membrane and for sealing the released membranes [43]. Since the high process temperatures $\left(900^{\circ} \mathrm{C}\right)$ make post-process $\mathrm{CMOS}$ integration impossible, through wafer vias have been developed to allow cMUTs to be flip-chip bonded to a signal-processing chip as mean for hybrid integration [44], [45]. A major drawback to this approach is the complicated fabrication process. A recently developed process based on PECVD silicon nitride generates a relatively large gap, $4000 \AA$ or larger, requiring high DC bias voltages, and the process temperature is somewhat high for $\mathrm{CMOS}$ integration, $400-500^{\circ} \mathrm{C}$, due an annealing step for the silicon nitride [46]. Noble et al. use a different approach to electronics integration which 
involves post-processing cMUTs directly over CMOS electronics [47]. This process makes use of polymer sacrificial layers under a silicon nitride membrane formed with plasma enhanced chemical vapor deposition (PECVD), but generates gaps in the $1-2 \mu \mathrm{m}$ range, which is not suitable for efficient cMUT operation at reasonable DC bias levels.

A recently developed approach to cMUT fabrication makes use of wafer bonding techniques to improve membrane uniformity [48]. However, the high temperatures required for bonding make post-process CMOS integration impossible. Another approach to electronics integration is to fabricate the cMUT out of the layers of a standard CMOS process [49]. Although this constitutes the ultimate in electronics integration, the cMUTs cannot be fabricated directly over the electronics, wasting valuable real estate on the chip.

In this research a low temperature, CMOS compatible cMUT fabrication process was developed which does not have any performance tradeoffs and has a reduced number of process steps as compared to hybrid integration using through wafer vias [50]. The only equipment required for the process consists of a PECVD system, a dry etching system, a metal sputtering system, a standard wet bench, and photolithograpy equipment. The process makes use of low-temperature PECVD processes for the deposition of the low stress silicon nitride structural layer at $250^{\circ} \mathrm{C}$, which is the maximum process temperature when a metal sacrificial layer is used. Alternatively, an amorphous silicon sacrificial layer deposited at $300^{\circ} \mathrm{C}$ can be used as the sacrificial layer. These process temperatures enable post-process CMOS electronics integration without compromising cMUT performance. Since a dielectric membrane is used, the electrode size and location can be changed to reduce parasitic capacitance and optimize performance [51], [52]. The membranes are sealed using PECVD silicon nitride, allowing for immersion operation 
and eliminating the need for long sealing channels required for LPCVD silicon nitride sealing. In the following sections, the details of the low temperature fabrication process are presented.

\section{CMUT Fabrication Process}

The cross section of an immersion cMUT fabricated using the low-temperature, CMOS compatible process is illustrated in Figure 53.

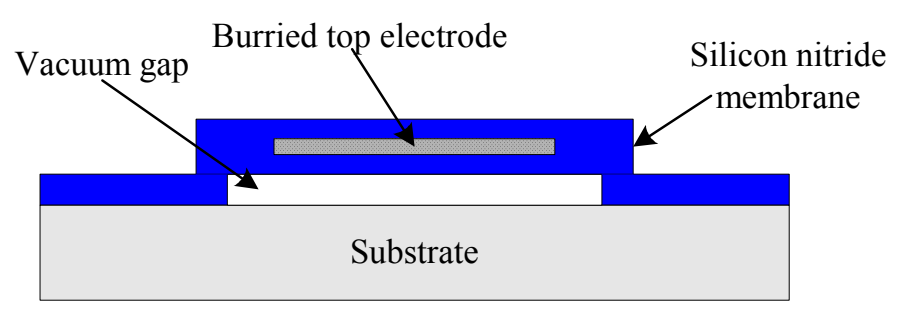

Figure 53 Schematic of completed immersion cMUT fabricated with the low temperature, CMOS compatible process.

The device consists of a silicon nitride membrane suspended above a vacuum sealed gap. Electrodes on the surface of the substrate and buried in the membrane allow electrostatic forces to be applied to the membrane. The fabrication process is illustrated in Figure 54 and will be described in the following sections. A detailed process description including the various recipes can be found in Appendix 3. 
(1)

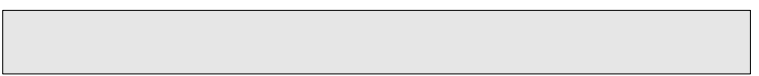

(2)

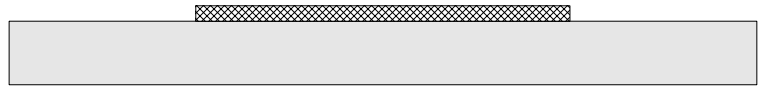

(3)

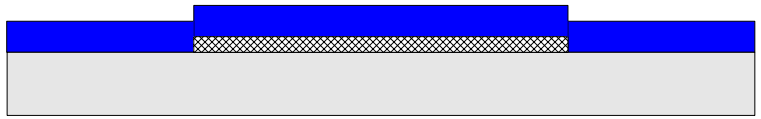

(4)

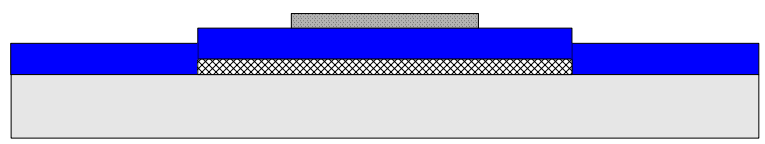

(5)

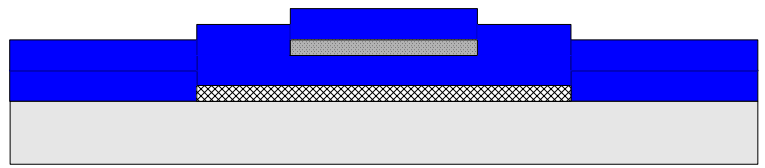

(6)

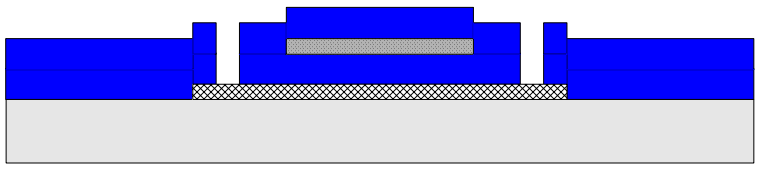

(7)

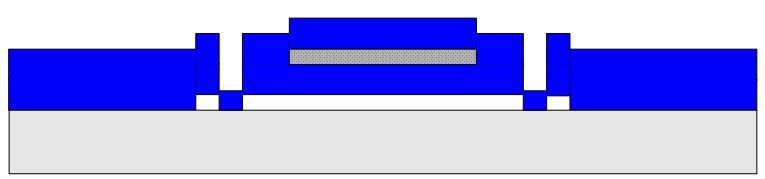

(8)

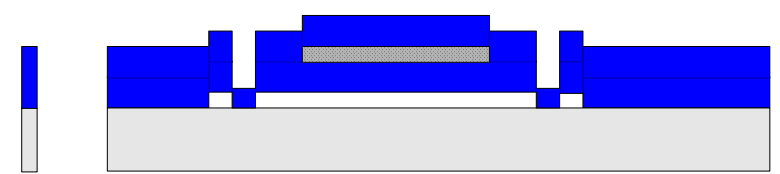

Figure 54 Illustration of low-temperature process flow for immersion cMUTs. The major steps are: (1) Bottom electrode formation, (2) Sacrificial layer formation, (3) Membrane deposition, (4)Top electrode formation, (5)Second membrane deposition, (6) Membrane release, (7) Membrane sealing, and (8) Via drilling 


\section{Bottom Electrode Formation}

The first step in the fabrication process forms the bottom electrode of the cMUT. The electrode can be formed by doping silicon wafers to a high conductivity, with a sheet resistance of less and $1 \Omega /$ square. Alternatively, low resistivity silicon wafers can be purchased directly from the supplier which eliminates the first step in the process.

\section{$\underline{\text { Sacrificial Layer Formation }}$}

The sacrificial layer, used to form the gap under the membrane, was formed out of either PECVD amorphous silicon or sputtered metal. Amorphous silicon was deposited at temperatures as low as $300^{\circ} \mathrm{C}$ and is patterned with reactive ion etching (RIE). The process parameters for the amorphous silicon deposition can be found in Table 4, while the etch recipe can be found in Table 5 in the section entitled Plasma Processing. The PECVD amorphous silicon had a low surface roughness (approximately $4 \AA$ ) which makes it a suitable sacrificial layer. The only disadvantage in using amorphous silicon was the poor selectivity of tetramethylamonium hydroxide (TMAH) used to release the membranes. The poor selectivity caused the silicon nitride forming the membrane to be thinned by a significant amount during the release process. Another disadvantage is that a silicon nitride isolation layer must be deposited before the sacrificial layer to protect the silicon substrate during the membrane release, increasing the number of process steps.

Sputtered metal was also used as a sacrificial layer and has several advantages over amorphous silicon. First, the maximum temperature of the process is reduced from $300^{\circ} \mathrm{C}$ to $250^{\circ} \mathrm{C}$. Second, chromium was found to be an excellent sacrificial layer because 
it has low roughness and is easily patterned with commercially available wet etchants and a photoresist masking layer. The chromium etchant is very selective to silicon nitride and is therefore a suitable release agent. Gaps as small as $1300 \AA$ have been fabricated using this chromium sacrificial layer, but this does not constitute the lower limit. In practice, the gap height is limited by the static deflection of the membrane due to atmospheric pressure and residual stresses, stiction, and the surface roughness of the sacrificial layer. The static deflection due to residual stresses can be overcome through proper design and the stiction problem can be overcome by releasing a thicker membrane and then thinning it back to the desired thickness after sealing. The surface roughness of the sacrificial layer is on the order of nanometers and represents the ultimate limit of the gap height. The top view of the mask and an image of the sacrificial layer are shown in Figure 55. 


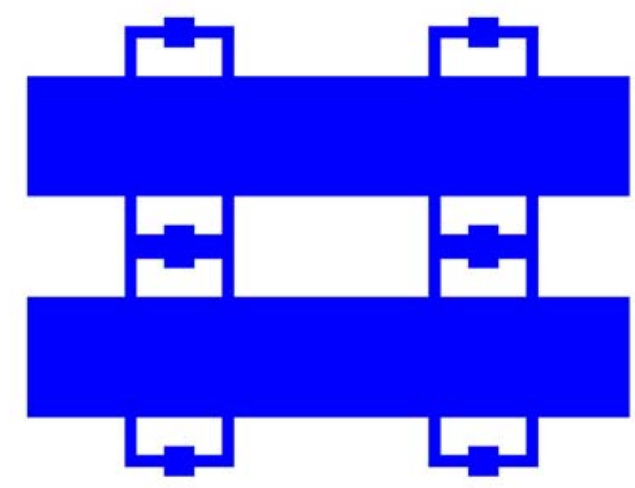

(a)

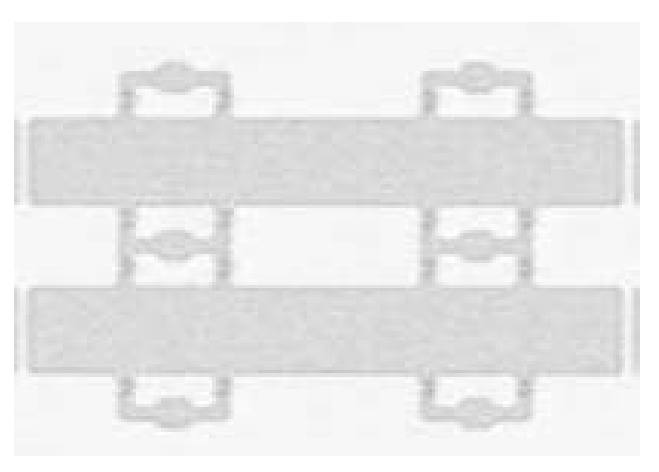

(b)

Figure 55 Mask image (a) and picture of chromium sacrificial layer (b). Each membrane is $100 \mu \mathrm{m}$ by $20 \mu \mathrm{m}$.

\section{Membrane Depostion}

Depositing PECVD silicon nitride over the sacrificial layer forms the vibrating membrane of the cMUT. The thickness of this layer varies depending upon the application, but $4000 \AA$ is a typical value. To prevent defects in this layer of silicon nitride, it was important to completely remove any photoresist residue before the deposition. When amorphous silicon was used as the sacrificial layer, a standard RCA or Piranha clean was used. However, when a metal sacrificial layer was employed, the 
standard cleaning processes (RCA or Piranha) could not be used because they removed the metal layer. Instead, the photoresist residue was removed by immersing the wafers in a beaker of acetone in an ultrasonic bath. The wafer was removed after soaking for 10$15 \mathrm{~min}$ and rinsed with acetone, methanol, and de-ionized water. After drying the wafer, the membrane silicon nitride could be deposited.

\section{Top Electrode Formation}

Aluminum was a poor choice for the top electrode material because it degraded during subsequent silicon nitride depositions, compromising the integrity of the passivation layer. Chromium is also a poor choice because of its high resistivity and high residual stress. However, the combination of the two materials produces a suitable top electrode. Depositing $1200 \AA$ of aluminum followed by $300 \AA$ of chromium using a DC sputterer formed the top electrode. The aluminum provides good electrical conductivity, and the chromium protects the aluminum from oxidation. Before depositing the metal electrode, contact pads were etched through the silicon nitride down to the silicon to allow ground connections to be made. After the deposition, the electrodes were patterned using wet aluminum and chromium etchants and a photoresist mask (Figure 56). 


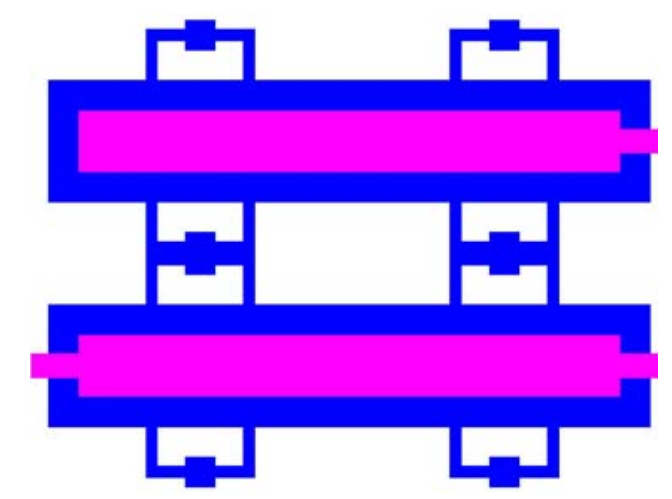

Figure 56 Mask image of patterned top electrode.

\section{$\underline{\text { Second Membrane Deposition }}$}

Another deposition of silicon nitride was carried out to increase the thickness of the membrane and protect the top electrode from the etchants used during release. This layer of silicon nitride was typically $4000 \AA$ thick.

\section{Membrane Release}

To allow the etchant to reach the sacrificial layer, holes were etched through the layers of silicon nitride using RIE. When an amorphous silicon sacrificial layer was used, it was necessary to carefully monitor the selectivity of the etch process to silicon. If the process has low selectivity, one can easily etch through the thin sacrificial layer, the thin silicon nitride isolation layer, and down to the silicon substrate. If this occurs, the silicon etchant used for release will attack the substrate and destroy the devices. The recipe used for selectively etching the silicon nitride can be found in Table 4 . When a metal sacrificial layer was used, the selectivity of the reactive ion etch is of little importance 
since the metal sacrificial layer acts as an etch stop. The etch holes are shown in Figure 57.

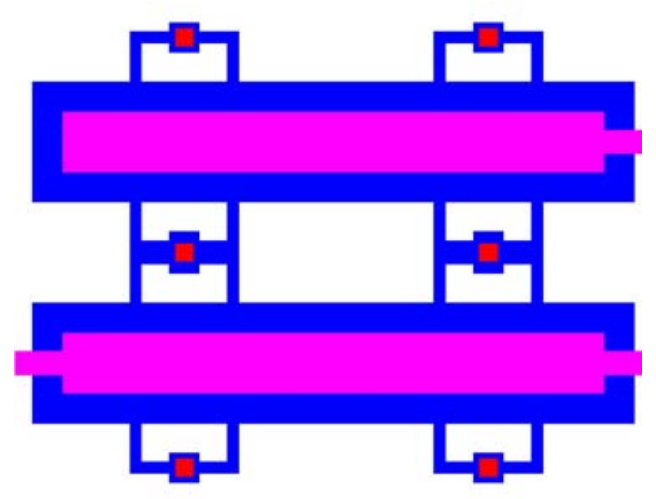

(a)

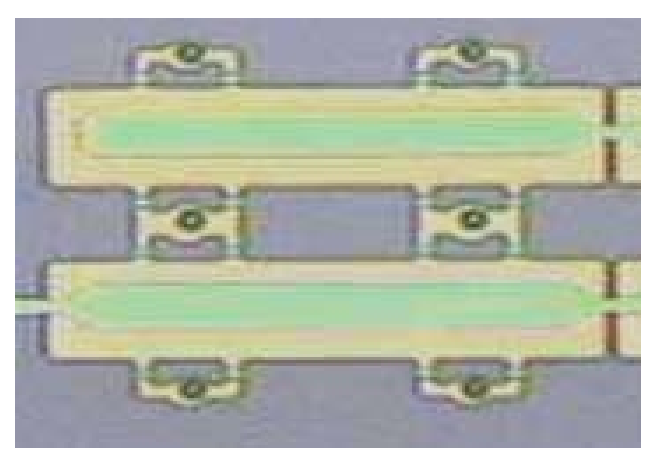

(b)

Figure 57 Mask image (a) and picture of etch holes used for releasing the membrane (b).

When using amorphous silicon, the release was performed by immersing the wafer in a beaker of $4 \mathrm{wt} \%$ TMAH doped with $13.5 \mathrm{~g} / \mathrm{L}$ of silicon. TMAH was used rather than $\mathrm{KOH}$ because of its CMOS compatibility, and the solution was doped to improve the selectivity to silicon nitride [53]. Although the etch rate of the silicon nitride was low, approximately $40-80 \AA / \mathrm{hr}$, it was significant during long releases which took $10-12 \mathrm{hrs}$. The slow attack of the silicon nitride resulted in a membrane with a non-uniform gap (Figure 58) which leads to device performance that is difficult to predict. 


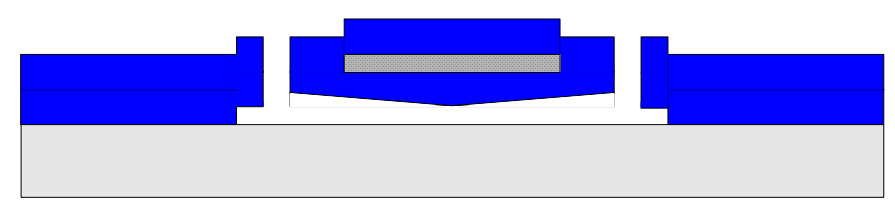

(a)

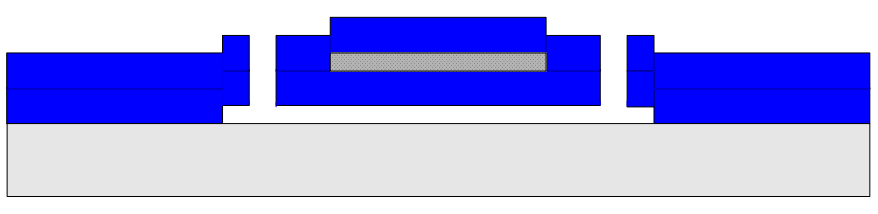

(b)

Figure 58 Illustration of non-uniform membrane gap (a) resulting from poor selectivity of the release agent. The gap thickness remains constant (b) when the chromium sacrificial layer is used due to the high selectivity of the chromium etch.

When the etch rate of silicon nitride was high $(80 \AA / \mathrm{hr})$, the etch rate of amorphous silicon was slower than normal, compounding the problem.

When chromium was used for the sacrificial material, commercially available chromium etch was used to release the membranes. The progression of the membrane release is shown in Figure 59. The etch rate of silicon nitride in the chromium etchant was too low to be measured, and thus the gaps resulting from the chromium sacrificial layer have a uniform thickness. With a uniform membrane thickness, the device performance can be more accurately modeled and predicted. 


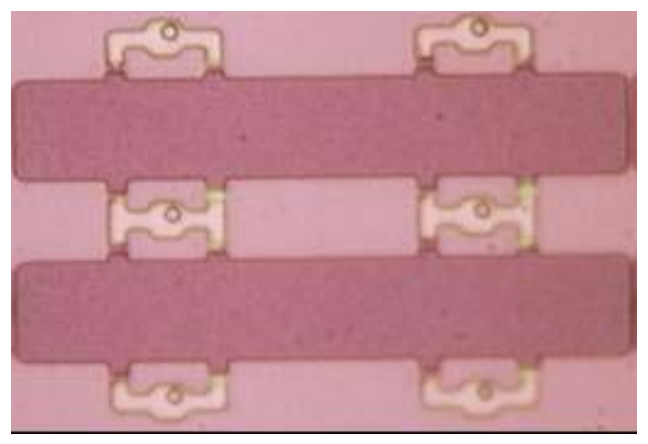

(a)

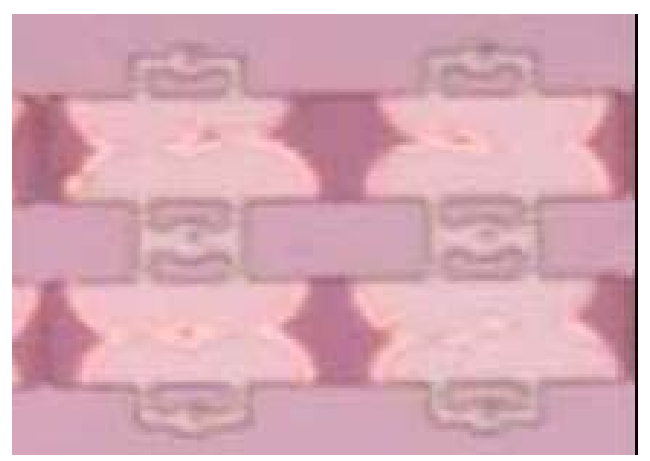

(b)

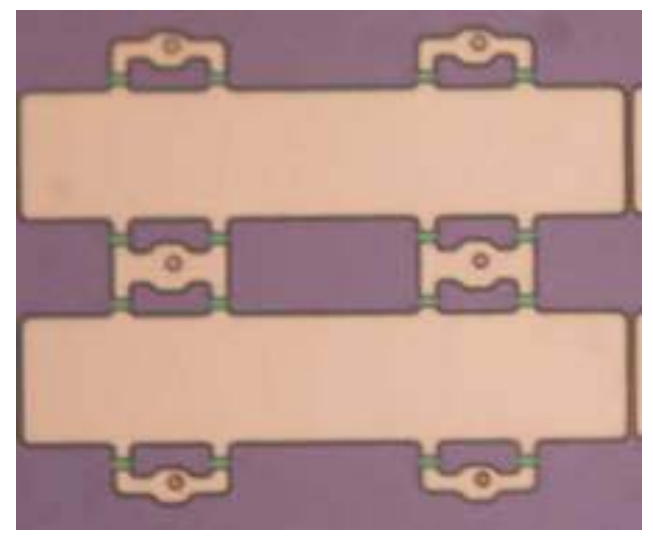

(c)

Figure 59 Illustration of the etch progression during membrane release. 


\section{Membrane Sealing}

The membranes were sealed for immersion applications by depositing another layer of PECVD silicon nitride. This silicon nitride sealing layer was typically much

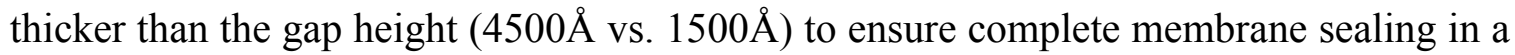
single deposition. CMUTs are traditionally sealed using LPCVD silicon nitride depositions, but this necessitates long etch channels to prevent deposits on the underside of the membrane. Since PECVD is much more directional than LPCVD silicon nitride, the membranes are sealed locally, in about $5000 \AA$ lateral distance, around the perimeter of the etch hole as seen in Figure 60.

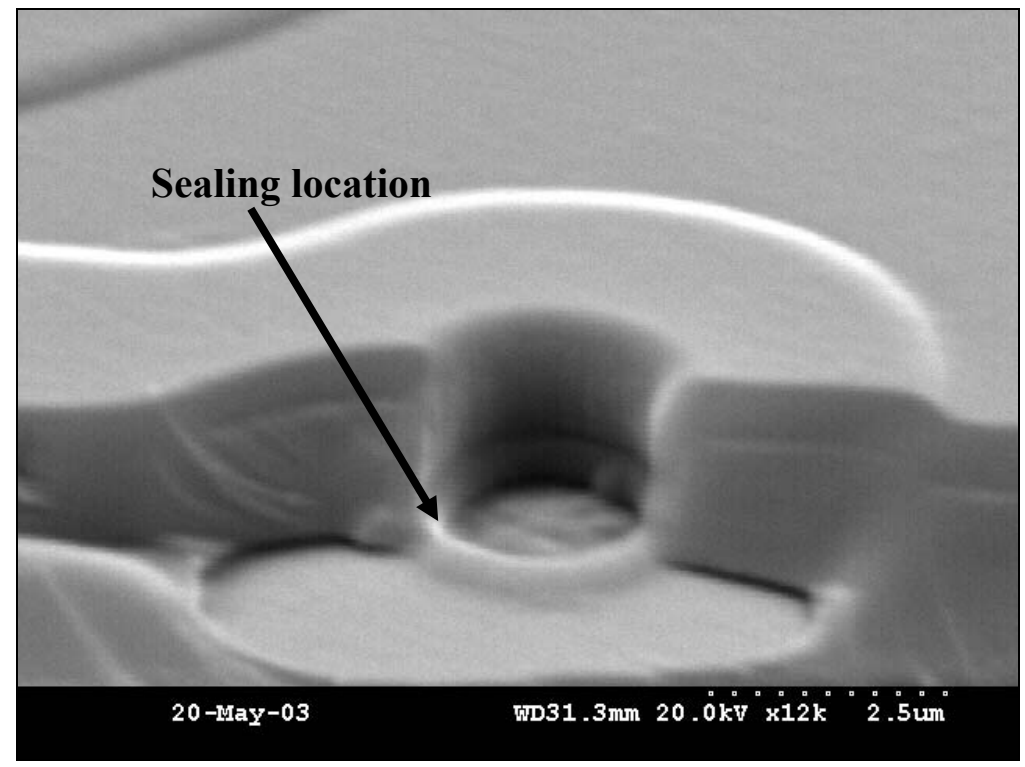

Figure 60 SEM showing the local sealing of the cMUT membrane around the etch hole resulting from a PECVD silicon nitride deposition.

In the figure, one can see the interface between the membrane silicon nitride $(10000 \AA)$ and the sealing layer $(4500 \AA)$. The local sealing eliminates the need for long sealing 
channels and allows membranes to be packed into denser arrays. Also, additional etch holes can be placed around the perimeter of the membrane to speed the release.

\section{Membrane Thinning}

After sealing the membranes, the thickness of the membrane was reduced to bring the resonant frequencies and collapse voltages to the desired values. A photoresist mask was used to protect the sealing locations while the membranes were thinned using an ICP (inductively coupled plasma) etcher. The recipe used for the etch is presented in Table 5. The RIE was initially used for this etch back step, but it produced a very rough surface which caused the electrodes to be corroded during operation. The mask and actual device are shown in Figure 61. 


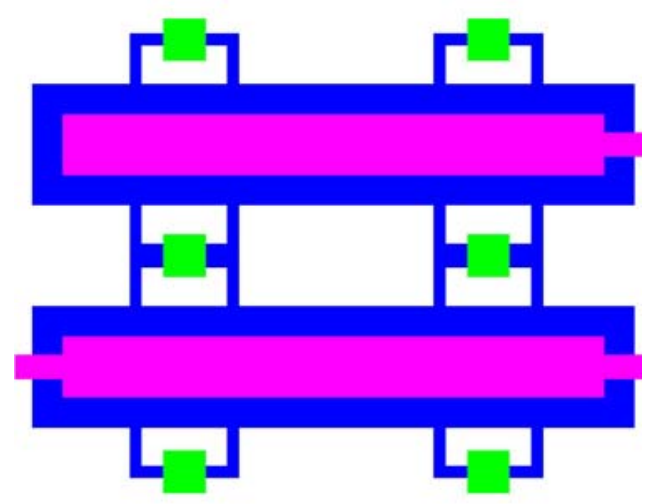

(a)

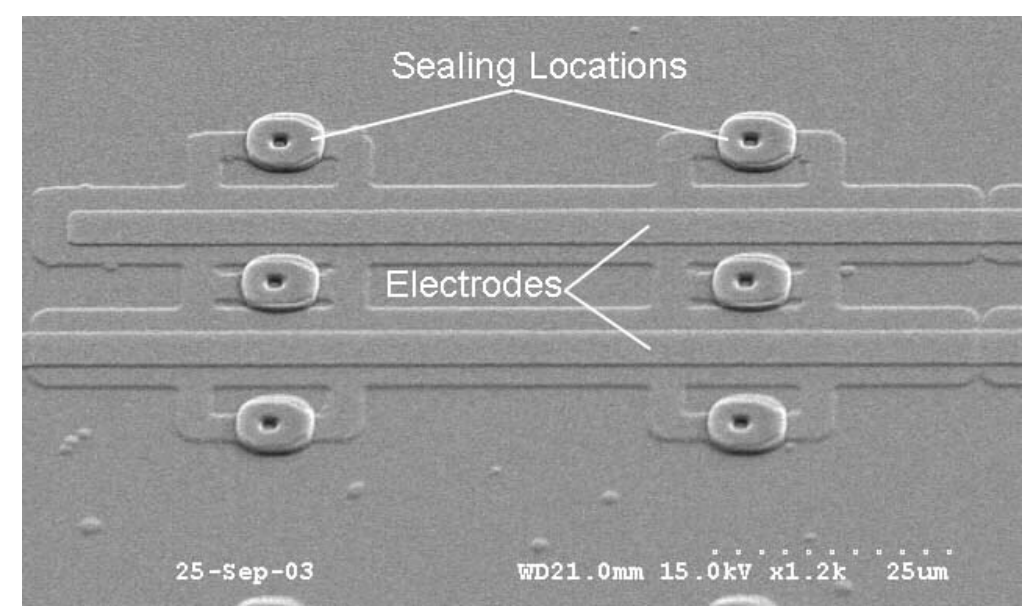

(b)

Figure 61 (a) Mask image and (b) scanning electron micrograph (SEM) of completed cMUT showing the protected sealing locations.

\section{Bond Pad Etch}

Before drilling the through wafer vias, the RIE was used to etch through the silicon nitride layer covering the bond pads. This allowed external electrical connections to be made with wire bonding. 


\section{Via Drilling}

Vias were etched through the silicon wafer to allow fluid connections to be made to microfluidic channels on the front of the wafer. The standard Bosch process $[54,55]$ was used to etch through the wafer while a thick photoresist mask protected the remainder of the wafer. The holes were quite large, $1 \mathrm{~mm}$ in diameter, and, therefore, the resolution of the lithography process was of little importance. The arrangement of the vias in relation to the transducers is shown in Figure 62.
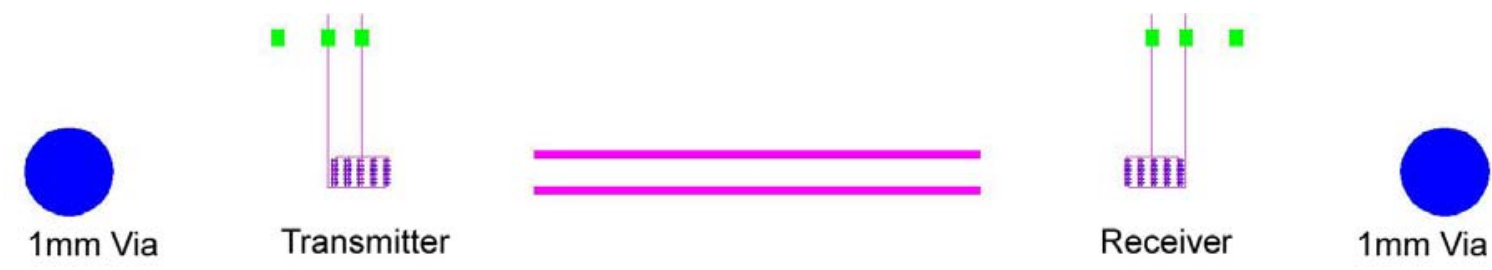

Figure 62 Mask image showing the location of the through-wafer vias. 


\section{Plasma Processing}

The depositions of silicon nitride and amorphous silicon were carried out in a single Unaxis 790 PECVD system [56], and the process parameters for both films are summarized in Table 4.

Table 4 Recipes for PECVD of silicon nitride and amorphous silicon.

\begin{tabular}{|c|c|c|}
\hline Parameter & Silicon Nitride & Amorphous Silicon \\
\hline $5 \% \mathrm{SiH} 4$ in He & $200 \mathrm{sccm}$ & $1000 \mathrm{sccm}$ \\
\hline $\mathrm{NH} 3$ & $8 \mathrm{sccm}$ & - \\
\hline $\mathrm{He}$ & $560 \mathrm{sccm}$ & - \\
\hline $\mathrm{N} 2$ & $150 \mathrm{sccm}$ & - \\
\hline Pressure & $1100 \mathrm{mTorr}$ & $900 \mathrm{mTorr}$ \\
\hline Power & $50 \mathrm{~W}$ & $50 \mathrm{~W}$ \\
\hline Temperature & $250^{\circ} \mathrm{C}$ & $300^{\circ} \mathrm{C}$ \\
\hline Deposition Rate & $80 \AA / \mathrm{min}$ & $75 \AA / \mathrm{min}$ \\
\hline
\end{tabular}

The silicon nitride and amorphous silicon films were patterned using the PlasmaTherm Reactive Ion Etcher (RIE) and the PlasmaTherm Inductivelty Couple Plasma (ICP) etcher in the MiRC Cleanroom. The process parameters are listed in Table

5.

Table 5 Recipes for reactive ion etch of silicon nitride and amorphous silicon

\begin{tabular}{|c|c|c|c|}
\hline Parameter & $\begin{array}{c}\text { Silicon Nitride } \\
\text { RIE }\end{array}$ & $\begin{array}{c}\text { Amorphous Silicon } \\
\text { RIE }\end{array}$ & Silicon Nitride ICP \\
\hline Etch Gas 1 & $45 \mathrm{sccm} \mathrm{CHF} \mathrm{CH}_{3}$ & $30 \mathrm{sccm} \mathrm{SiCl}_{4}$ & $30 \mathrm{sccm} \mathrm{CF}_{4}$ \\
\hline Etch Gas 2 & $5 \mathrm{sccm} \mathrm{\textrm {O } _ { 2 }}$ & $20 \mathrm{sccm} \mathrm{Cl}_{2}$ & - \\
\hline Pressure & $40 \mathrm{mTorr}$ & $30 \mathrm{mTorr}$ & $5 \mathrm{mTorr}$ \\
\hline Power & $200 \mathrm{~W}$ & $150 \mathrm{~W}$ & $50 \mathrm{~W} \mathrm{RIE}, 400 \mathrm{~W}$ ICP \\
\hline Etch Rate & $650 \AA / \mathrm{min}$ & $500 \AA / \mathrm{min}$ & $2200 \AA / \mathrm{min}$ \\
\hline
\end{tabular}

The process for etching silicon nitride in the RIE etched nitride approximately 5 times faster than amorphous silicon, while the process for amorphous silicon etched silicon 
approximately 15 times faster than silicon nitride. The ICP etch process for silicon nitride had a selectivity to photoresist of approximately 1:1.

\section{$\underline{\text { Silicon Nitride Film Characterization }}$}

All relevant properties of the PECVD silicon nitride were measured to fully characterize the transducers, and the results are summarized in Table 6. The residual stress was determined using the wafer bow technique [57]. A Veeco Dektak 3030 profilometer was used to measure the wafer bow before and after depositing a layer of silicon nitride. The residual stress was determined to be tensile with a value of $35 \pm$ $5 \mathrm{MPa}$ on several wafers from different process runs. The density was determined to be $2040 \pm 20 \mathrm{~kg} / \mathrm{m}^{3}$ by weighing a silicon wafer before and after depositing a known thickness of silicon nitride. The density is at the very low end of the published values and indicates that the film has high porosity. This assumption is further verified by the measured relative permittivity of 6.3 , which is lower than the high density silicon nitride films.

The Young's modulus of a $2 \mu \mathrm{m}$ layer of silicon nitride was determined to be around 160GPa using nanoindentation. However, measurements on cMUTs suggest a $30 \%$ lower value when experimental results are consistently fit with finite element analysis (FEA) on devices with various dimensions. Since the literature suggest that the Young's modulus of PECVD silicon nitride would be around 160GPa with a density of $2600 \mathrm{~kg} / \mathrm{m}^{3}$ and decrease with the density of the film, we believe that the lower value obtained from electromechanical measurements are more reliable [58], [59]. 
Table 6 Properties of silicon nitride film.

\begin{tabular}{|c|c|}
\hline Property & Value \\
\hline Residual Stress & $35 \pm 5 \mathrm{MPa}(\mathrm{T})$ \\
\hline Density & $2040 \pm 20 \mathrm{~kg} / \mathrm{m}^{3}$ \\
\hline Young's Modulus & $160 \pm 10 \mathrm{GPa}$ (Nanoindentation) \\
& $110 \mathrm{GPa}$ (FEA fit to experimental data) \\
\hline Relative Dielectric Constant & $6.3 \pm 0.1$ \\
\hline
\end{tabular}

\section{$\underline{\text { Discussion }}$}

The fabrication process developed for cMUTs represents an improvement over existing processes in several regards. The low processing temperature of $250^{\circ} \mathrm{C}$ is $150^{\circ} \mathrm{C}$ lower than other reported values and facilitates future electronics integration by postprocessing cMUTs over CMOS electronics. For sub-micron CMOS electronics, the thermal budget must be monitored carefully to maintain the performance of the electronics. Therefore, the lowest process temperate attainable is desired. Post-CMOS electronics integration is the most attractive approach since economies of scale can be obtained by using established CMOS foundries for producing the electronics [60]. Additionally, the process makes use of standard microelectronic processing equipment which is available in nearly all cleanroom facilities. Finally, the PECVD sealing has been found to effectively vacuum seal the gap under the transducer so that the transducers can be used for immersion applications. The localized nature of the sealing allows transducers to be packed into dense arrays for improved acoustic coupling to the fluid. 


\section{CHAPTER 6}

\section{ELECTRICAL CHARACTERIZATION}

Before delving into the details of the electrical performance of cMUT arrays, it is helpful to review the operation of a single electrostatically actuated membrane. After establishing an understanding of cMUT operation, the equivalent circuit model can be presented to illustrate the importance of accurate measurements of a transducer's electrical impedance. Finally, the electrical performance of interdigital cMUT arrays under different loading conditions is presented.

\section{Electrostatic Actuation of cMUT Membranes}

As shown in Figure 6, a single cMUT membrane consists of a deformable membrane which suspends a moveable electrode over an electrically conductive substrate. This system can be modeled to first order as a mass, and a linear spring in series with a parallel plate capacitor as shown in Figure 63 [61].

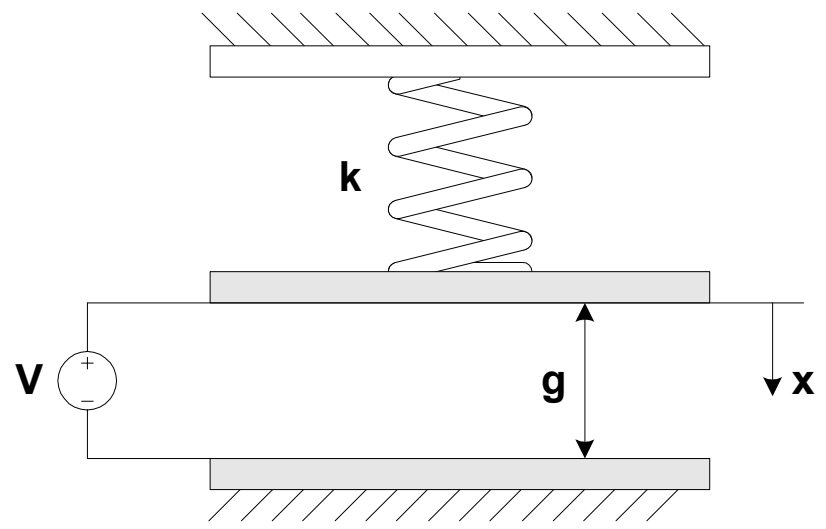

Figure 63 Illustration of first-order model of a cMUT. 
The spacing between the plates of the capacitor is denoted as $g$, and the initial gap thickness as $g_{0}$. The deflection of the moveable top plate is defined as $x$, with the positive direction pointing towards the fixed electrode. When a constant voltage is applied between the electrodes, the top electrode is deflected due to electrostatic forces. Under stable conditions, the electrostatic force is balanced by the restoring force in the spring.

The electrostatic force acting on a parallel place capacitor is determined using the principal of virtual work to be the gradient of the electrostatic energy between the plates,

$$
\vec{F}_{v}=\nabla W_{e}
$$

The electrostatic energy for a parallel plate capacitor is given by

$$
W_{e}=\frac{1}{2} C V^{2}
$$

where $C$ is the capacitance between the plates and $V$ is the applied DC voltage. The capacitance is dependent on the gap height, $g$, the membrane thickness, $t_{m}$, and the dielectric properties of the membrane and vacuum sealed gap. The cross section of cMUT membrane is shown in Figure 64 to illustrate the geometry.

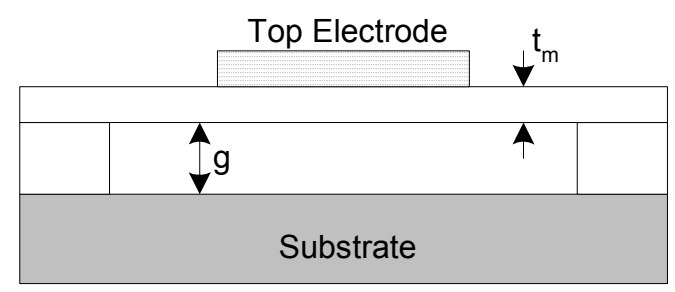

Figure 64 Schematic of cMUT membrane. 
The capacitance between the fixed bottom electrode and the moveable top electrode can be written as

$$
C=\frac{\varepsilon_{r} \varepsilon_{0} A}{t_{m}+\varepsilon_{r} g}
$$

where $\varepsilon_{0}$ is the permittivity of free space and $A$ is the cross sectional area of the plate. The electrostatic force can then be written as

$$
\left(F_{v}\right)_{x}=\frac{\partial}{\partial x}\left(\frac{1}{2} \frac{\varepsilon_{r} \varepsilon_{0} A}{t_{m}+\varepsilon_{r} g} V^{2}\right)=-\frac{\varepsilon_{0} \varepsilon_{r}^{2} A V^{2}}{2\left(t_{m}+\varepsilon_{r} g\right)^{2}} .
$$

The net force acting on the membrane is the result of the electrostatic force and the restoring force of the spring, $k x$,

$$
F_{n e t}=\frac{\varepsilon_{0} \varepsilon_{r}^{2} A V^{2}}{2\left(t_{m}+\varepsilon_{r} g\right)^{2}}-k\left(g_{0}-g\right)
$$

where $\mathrm{x}$ has been replaced by $g_{0^{-}} g$. For the system to be stable, with a nonzero $g$, the differential force, $d F_{n e t}$, must be greater than zero,

$$
d F_{n e t}=\frac{\partial F_{n e t}}{\partial g} d g=\left(-\frac{\varepsilon_{0} \varepsilon_{r}^{2} A V^{2}}{\left(t_{m}+\varepsilon_{r} g\right)^{3}}+k\right) d g>0 \Rightarrow k>\frac{\varepsilon_{0} \varepsilon_{r}^{2} A V^{2}}{\left(t_{m}+\varepsilon_{r} g\right)^{3}}
$$

As the voltage is increased, a point is reached where the electrostatic force equals the restoring force in the spring. If the voltage is further increased, the electrostatic force is greater than the restorative force in the spring, and the top plate is pulled-in to the bottom plate. This point is known as the pull-in or collapse point. The gap, $g_{P I}$, and voltage, $V_{P I}$, at the pull-in point can be determined by setting $d F_{n e t}$ and $F_{\text {net }}$ equal to zero and solving for $g$ and $V$. The resulting pull-in gap height and voltage are

$$
g_{P I}=\frac{2 g_{0}}{3}
$$

and 


$$
V_{P I}=\sqrt{\frac{8 k g_{0}^{3}}{27 \varepsilon_{0} A}}
$$

From Equation 62, one can see that the maximum deflection of the top electrode is one third of the gap height.

Now, it is interesting to investigate the behavior of the spring-capacitor system when an $\mathrm{AC}$ voltage, $V_{A C}$, is applied in addition to the $\mathrm{DC}$ voltage, $V_{D C}$. If the displacement, $x$, is assumed to be small compared with the initial gap thickness, $g_{0}$, one finds that the force resulting from the small change in voltage, $F_{v}$, can be written as

$$
F_{v} \approx \frac{\varepsilon_{0} \varepsilon_{r}^{2} A V^{2}}{2\left(t_{m}+\varepsilon_{r} g\right)^{2}} \propto V^{2}
$$

where $V$ is the sum of the $\mathrm{AC}$ and $\mathrm{DC}$ voltages. If the $\mathrm{AC}$ voltage is written as a time harmonic function,

$$
V_{A C}=V_{0} \cos (\omega t)
$$

and the square of the total voltage is calculated, one finds that

$$
V^{2}=V_{D C}^{2}+2 V_{D C} V_{0} \cos (\omega t)+V_{0}^{2} \cos ^{2}(\omega t)
$$

If Equation 66 is substituted into Equation 64 and a trigonometric identity is used for $\cos ^{2}(\omega t)$, the force acting on the membrane due to the AC and DC voltages can be written as,

$$
F_{v}=\frac{\varepsilon_{0} \varepsilon_{r}^{2} A}{2\left(t_{m}+\varepsilon_{r} g\right)^{2}}\left[\left(\frac{1}{2} V_{0}+V_{D C}^{2}\right)+2 V_{D C} V_{0} \cos (\omega t)+V_{0} \cos (2 \omega t)\right]
$$

From Equation 67, one can see the force acting on the top electrode has a DC component, an $\mathrm{AC}$ component with frequency $\omega$, and another $\mathrm{AC}$ component with frequency $2 \omega$, the second harmonic. To linearize the response of the system, it is desirable to minimize the 
second harmonic. This is done by operating at a point where the DC voltage is much greater than the AC voltage, $V_{D C}>>V_{A C}$. Since the second harmonic amplitude is not dependent on the DC bias voltage, it suppressed when a large bias voltage is applied. The maximum DC voltage that can be applied is the collapse voltage, and thus the system should be operated very near this pull-in point.

\section{$\underline{\text { Equivalent Circuit Model }}$}

The equivalent circuit model for a cMUT is a useful tool for modeling the overall performance of the transducer. The small signal equivalent circuit model for a cMUT is based on the general equivalent circuit developed by Hunt [62] and is shown in Figure $65[63],[64]$.

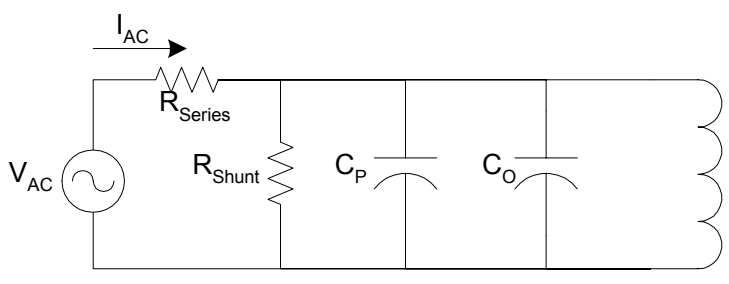

Electrical

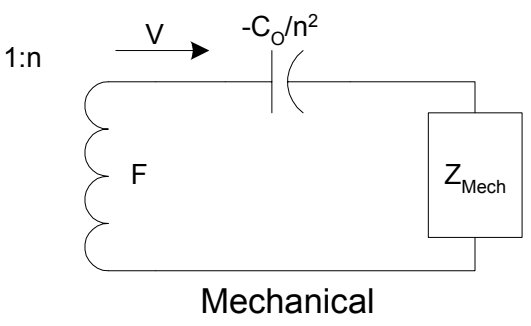

Figure 65 Equivalent circuit model of a cMUT.

The equivalent has two domains: electrical and mechanical. When the cMUT operates as a transmitter, the goal is to convert electrical energy from the $\mathrm{AC}$ voltage source to mechanical energy of the vibrating membrane. As a receiver, the goal is to convert the velocity of the membrane in to a measureable change in voltage. The energy transformation is represented by the transformer with ratio $1: n$. The various elements of the equivalent circuit will now be discussed. 
On the electrical side, the two dissipative elements are the series and shunt resistances. The series resistance, $R_{\text {series }}$, represents any electrical losses in the electrical wires of the system owing to the fact that they are not perfect conductors. The shunt resistance, $R_{\text {shunt }}$, represents a possible electrical path through the membrane to ground. Both of these resistances can be neglected since the electrical losses in the electrical leads are small, and the conductance through the membrane is very small.

The two storage elements on the electrical side of the equivalent circuit are the parasitic and device capacitances. The parasitic capacitance, $C_{p}$, represents any stray capacitance resulting from the overlap of electrical wires and ground. For transmission operation, $C_{p}$ is of little concern since a large amount of power is input into the system. However, for reception it is desirable to minimize the parasitic capacitance so that changes in the device capacitance resulting from the motion of the membrane can be measured. The device capacitance, $C_{0}$, is the static capacitance of the cMUT membrane when a DC bias is applied. This capacitance is found by substituting the DC bias voltage into the relation for the cMUT capacitance (Equation 58).

The transformer ratio, $n$, can be determined by determining the small signal force acting on the membrane which results from the $\mathrm{AC}$ voltage. If the force acting on the membrane is linearized by applying a large DC bias voltage, the oscillating component of Equation $67, F_{A C}$ can be written as

$$
F_{A C}=\frac{\varepsilon_{0} \varepsilon_{r}^{2} A V_{D C}}{\left(t_{m}+\varepsilon_{r} g\right)^{2}} \cdot V_{0} \cos (\omega t)
$$

In the preceding equation, the $\mathrm{AC}$ voltage is multiplied by a constant to obtain the oscillating force acting on the membrane. This constant is called the transformer ratio since it transforms a voltage to a force and is written as, 


$$
n=\frac{\varepsilon_{0} \varepsilon_{r}^{2} A V_{D C}}{\left(t_{m}+\varepsilon_{r} g\right)^{2}} .
$$

The transformer ratio should be maximized to obtain the largest force acting on the membrane for a given $\mathrm{AC}$ voltage input. For a fixed DC bias voltage, the transformer ratio has the following proportionality,

$$
n \propto \frac{1}{t_{m}^{2}}, \frac{1}{g^{2}} .
$$

Thus, $n$ is maximized when the membrane thickness and gap are minimized. However, the membrane thickness is dictated by the desired resonant frequency of the membrane. Therefore, the gap height has the greatest effect on the transformer ratio.

Since the cMUT is biased to near collapse during operation, it is of interest to determine how the transformer ratio at collapse scales. If the values for the gap height and voltage at the pull-in point are substituted into the transformer ratio, one finds that

$$
n=\varepsilon_{0} \varepsilon_{r} \sqrt{\frac{8 k A}{27 \varepsilon_{0}}} \cdot \frac{g_{o}^{3 / 2}}{\left(t_{m}+\frac{2}{3} \varepsilon_{r} g_{0}\right)^{2}} .
$$

If again the membrane geometry is held constant, the transformer ratio is found to be proportional to $1 / \sqrt{g_{0}}$. Thus, reducing the initial gap height results in a more efficient transducer.

In summary, there are two key reasons to apply DC bias to the membranes of a cMUT. First, the large DC voltage linearizes the response of the system by suppressing the second harmonic. Second, increasing the DC bias voltage increases the transformer ratio so that more electrical energy is converted to acoustic energy, and vice-versa. 
The mechanical side of the equivalent circuit has a storage element and an impedance element. The presence of the storage element can be determined by investigating the differential forces acting on the membrane. If the oscillating membrane deflection is much smaller than the vacuum gap, the differential force can be written as

$$
d F=n \cdot d V-\frac{n^{2}}{C_{0}} d g
$$

where $C_{0}$ is the static device capacitance. The differential change in gap height, $d g$, is equal to the membrane displacement, $x$. The displacement of the membrane is related to the velocity by

$$
x(t)=\int v(t) d t .
$$

This allows the differential force, $d F$, to be written as

$$
F_{A C}=n \cdot V_{A C}-\frac{n^{2}}{C_{0}} \int v(t) d t
$$

where $d F$ has been replaced with $F_{\mathrm{AC}}$ and $d V$ has been replaced with $V_{\mathrm{AC}}$. Recall that for a capacitor, the voltage is related to the current by

$$
V(t)=\frac{1}{C} \int_{-\infty}^{t} i(\tau) d \tau .
$$

For a mechanical system, the current is analogous to velocity and voltage is analogous to force. Based on this analogy, the term on the far right in Equation 72 can be viewed as an energy storage element similar to a capacitor. The capacitance of this element is

$$
C=-\frac{C_{0}}{n^{2}} .
$$


The other component on the mechanical side of the equivalent circuit is the mechanical impedance. This impedance is typically modeled as two elements in series as shown in Figure 66.

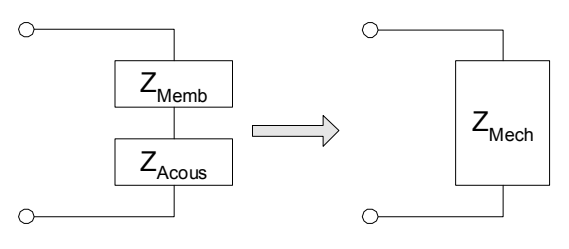

Figure 66 Standard representation of the mechanical impedance of a cMUT membrane.

The impedance of the membrane, vibrating in vacuum, is placed in series with the radiation impedance of the membrane when immersed in fluid. The impedance of the membrane is determined by finding the average velocity of the membrane resulting from excitation by a harmonic pressure. Typically, the radiation impedance term comes from the result for a baffled piston. However, this result is not valid when there are other vibrating membranes in the near field which is the case for a cMUT array. Therefore, finite element models can be used to determine the overall mechanical impedance of the membrane vibrating in a fluid.

\section{Impedance Measurements}

The first step in characterizing the operation of a cMUT is to measure the electrical impedance. This provides important information such as the resonant frequency of the membranes and their collapse voltage. The impedance is measured using the system illustrated in Figure 67. 


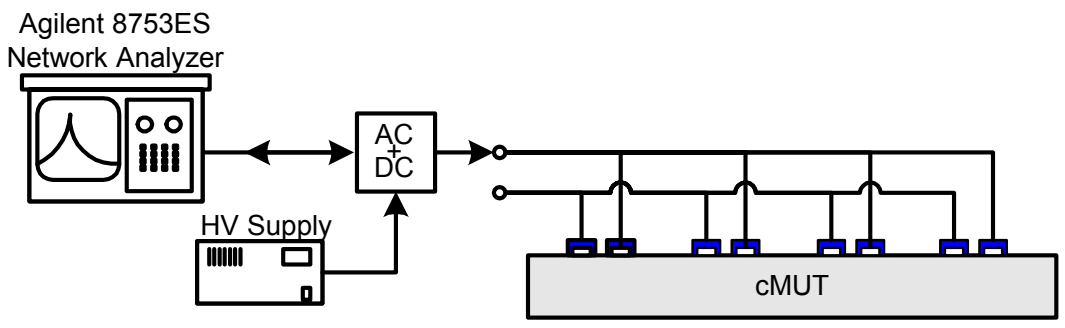

Figure 67 Schematic of setup used for standard impedance measurements.

The network analyzer measures the complex impedance of the transducer by measuring the electrical reflection coefficient and converting this to a complex electrical impedance. The instrument can measure the impedance at a single frequency or perform a linear frequency sweep over a specified range. The frequency sweep is useful for determining the frequency at which the membranes of the cMUT resonate, the capacitance of the transducer and the collapse voltage. The impedance of a cMUT in air was measured at several voltages for a $10 \mathrm{MHz}$ device with 10 finger pairs, and the results are shown in Figure 68 . The collapse voltage of this device was approximately $110 \mathrm{~V}$. 


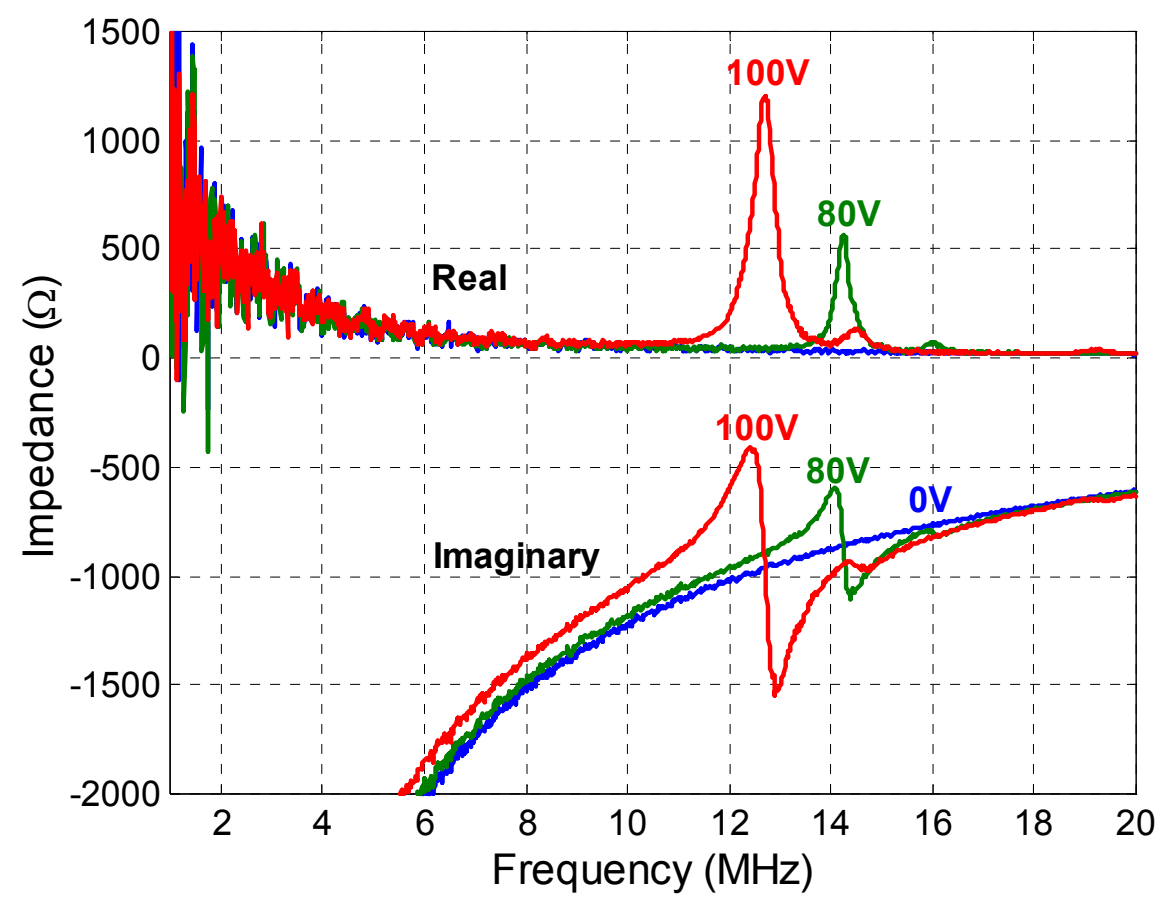

Figure 68 Complex impedance of a cMUT in air at bias voltages of 0,80 , and $100 \mathrm{~V}$.

In Figure 68, one can see that as the DC bias voltage is increased, the resonance of the membrane becomes more pronounced. Also, the resonant frequency of the transducer shifts to a lower frequency due to electrostatic spring softening [63]. This effect results from the competition between the linear restoring force of the membrane and the nonlinear electrostatic force. As the bias voltage is increased, the electrostatic force increases faster than the spring-like force of the membrane. The result is that the effective spring constant of the membrane is reduced, thus decreasing the resonant frequency. When the collapse voltage is reached, the amplitude of the resonant peak is decreased since the motion of the membrane is significantly reduced.

A modal analysis was performed using ANSYS to verify the resonant frequency of the unbiased membranes. The membrane was modeled in three dimensioned with the 
measured material properties for the silicon nitride membrane. The model predicted that the first resonant frequency of the membrane would be $15.3 \mathrm{MHz}$ when the Young's Modulus had a value of $120 \mathrm{GPa}$, which is well within the experimentally measured range. This resonance corresponds to the first mode commonly referred to as the "breathing" mode and is shown in Figure 69.

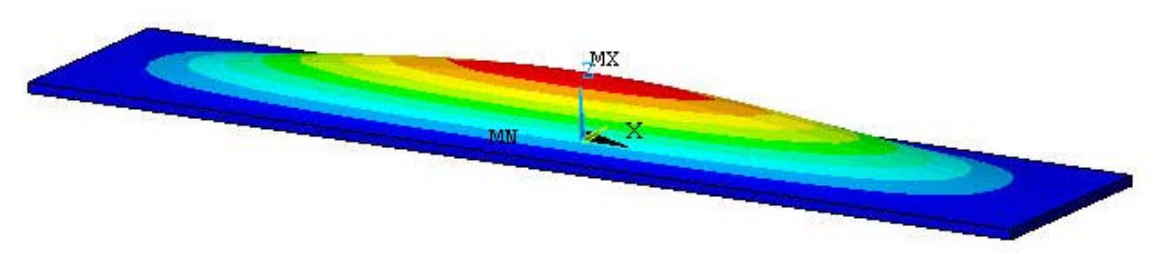

Figure 69 Mode shape of $100 \mu \mathrm{m}$ long, $20 \mu \mathrm{m}$ wide, $0.8 \mu \mathrm{m}$ thick silicon nitride membrane resulting from a modal analysis in ANSYS.

This mode shape is the most useful for transmitting an acoustic wave since it has the largest net volume displacement. From the impedance data, the resonant frequency when the membrane is far from collapse corresponds to free resonance of the structure. The resonant frequency under these low voltage conditions was determined to be $15.25 \mathrm{MHz}$ which is quite close to the FEM predictions.

The imaginary impedance in Figure 68 is dominated by the capacitance of the transducer. The shape of the curve can be predicted by the equation for the reactance of an ideal capacitor,

$$
X_{C}=\frac{1}{j \omega C}
$$

where $C$ is the capacitance and $\omega$ is the angular frequency. As the DC bias is increased, the capacitance is also increased, thus reducing the reactance, as seen in Figure 68. 
When the transducer is immersed in a water half-space, the resonant frequency of the membrane is decreased due to the mass loading of the fluid, as shown in Figure 70.

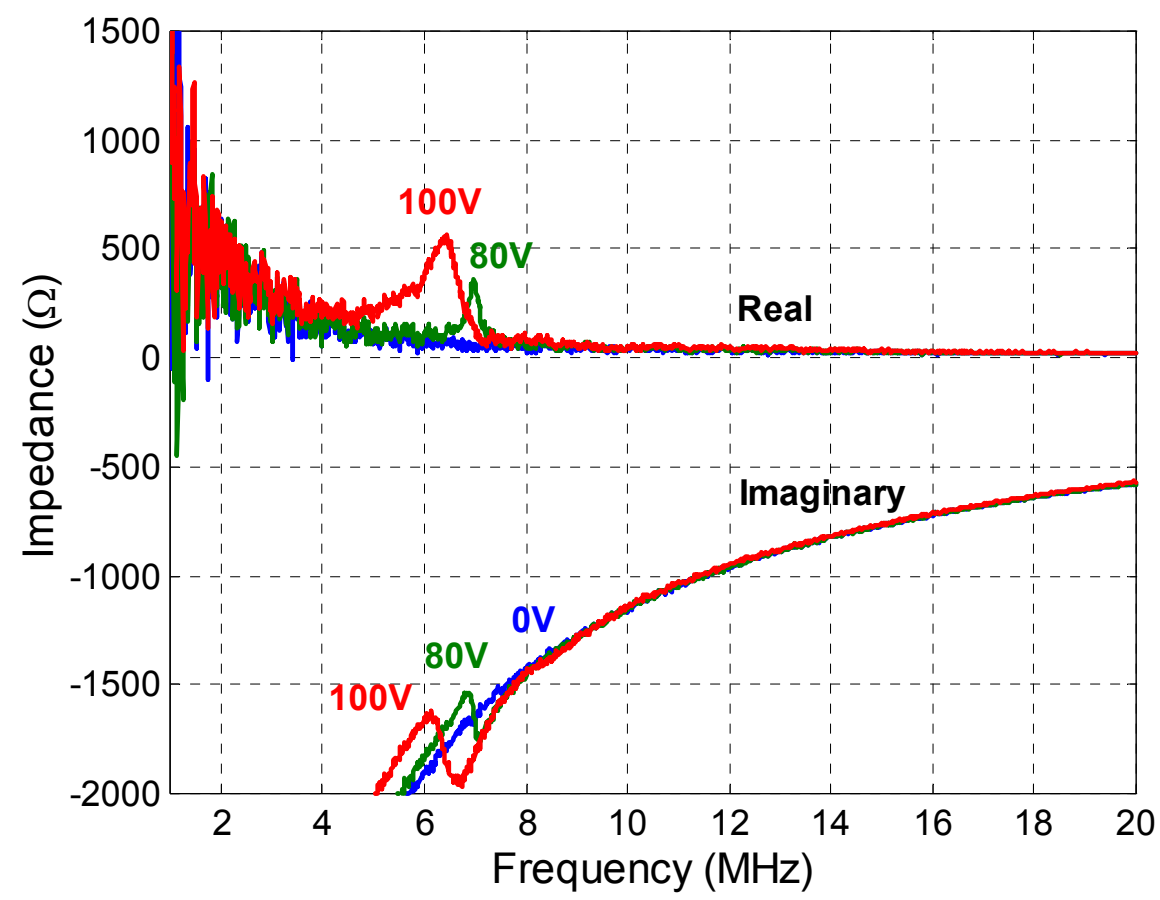

Figure 70 Complex impedance of a cMUT in water at bias voltages of 0,80 , and $100 \mathrm{~V}$.

In Figure 70, one can also see that the amplitude of the resonant peak is decreased due to the mass loading. The resonant frequency has also decreased from $12.7 \mathrm{MHz}$ in air to 6.4MHz in water, for a DC bias of $100 \mathrm{~V}$.

Ideally the transducer should operate at the fluid-loaded resonant frequency to couple a large amount of power to the fluid. However, even at resonance, the imaginary part of the impedance is much larger than the real part which causes a large amount of electrical power to be reflected when the transducer is connected to $50 \Omega$ electronics. To increase the power delivered to the fluid, the magnitude of the imaginary impedance can be decreased by placing an inductor in series with the cMUT. The magnitude of the 
inductance is determined by measuring the imaginary impedance at the frequency of interest. The electrical impedance of an inductor, $X_{L}$, can be written as

$$
X_{L}=j \omega L
$$

where $\omega$ is the angular frequency and $L$ is the inductance. Small, chip inductors must be used for tuning the cMUTs to obtain the desired frequency response.

The imaginary impedance should be tuned to zero so that no power is stored in the transducer at the operating frequency. The inductively tuned impedance of the same transducer measured in Figure 68 and Figure 70 was measured and is plotted in Figure 71. In Figure 71, one can see that the imaginary impedance goes through zero at a frequency of approximately $10.2 \mathrm{MHz}$, which was the desired operating point of the transducer.

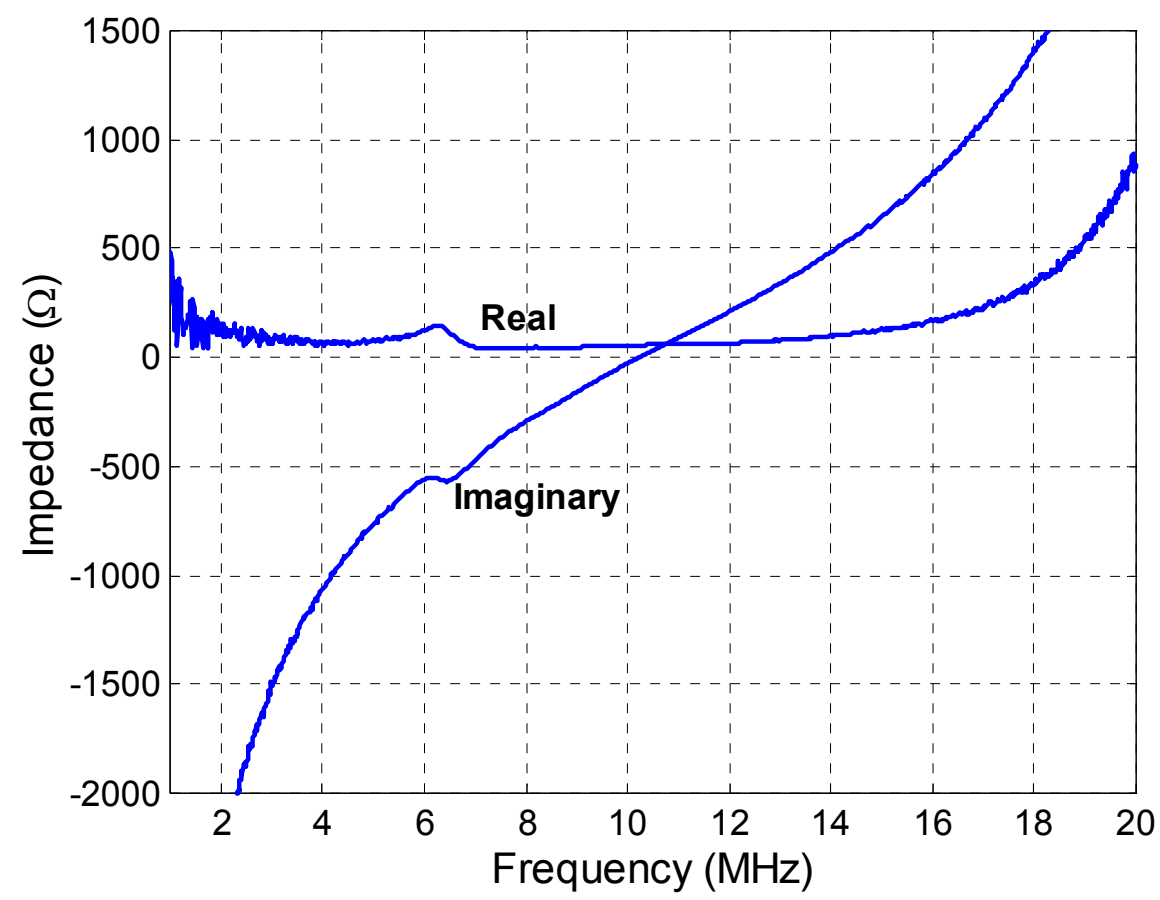

Figure 71 Complex impedance of tuned cMUT in water half-space with 100V DC bias. 
The increase in power delivered to the transducer can be determined by calculating at the power transmission coefficient, which is defined as the ratio of the transmitted power to the incident power. The equivalent circuit used in this calculation is shown in the following figure.

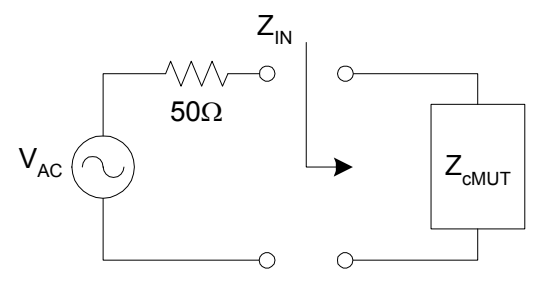

Figure 72 Equivalent circuit used for calculating the transmission coefficient.

An analysis of this circuit produces the following equation for the power transmission coefficient, $\tau$,

$$
\tau=\frac{4 Z_{c M U T} \cdot 50 \Omega}{\left(50 \Omega+Z_{c M U T}\right)^{2}} .
$$

The maximum power is delivered to the transducer when $\tau$ is equal to 1 , which occurs when $Z_{c M U T}$ is equal to $50 \Omega$. As an example, the power transmission coefficient for the tuned and untuned device used the experimental results of Figure 68 and Figure 70 was calculated. The untuned device had a power transmission coefficient of $0.023+\mathrm{j} 0.177$ at a frequency of $10.5 \mathrm{MHz}$. Electrical tuning increased the transmission coefficient to $0.997+\mathrm{j} 0.004$, which is an increase of $98 \%$. This enhancement of power delivered to the transducer, and ultimately to the fluid, is a necessity for ultrasonic fluid actuation since the amplitude of the pressure wave generated by the transducer needs to be as large as possible to obtain reasonable flow rates from acoustic streaming. 
The power consumed by an interdigital cMUT in a water filled microchannel was determined by measuring the electrical impedance with DC bias levels of 0 and $100 \mathrm{~V}$ (Figure 73).

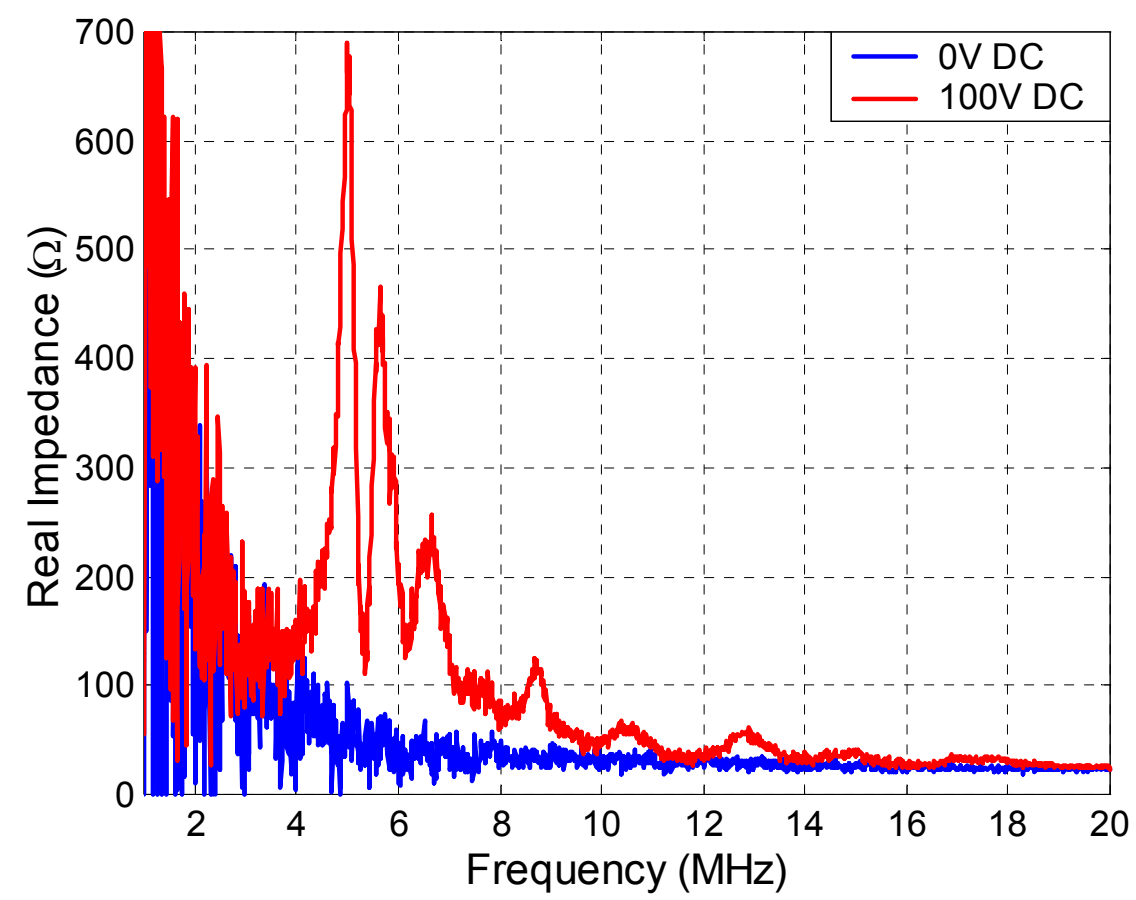

Figure 73 Real impedance of interdigital cMUT placed in water-filled microchannel at different levels of DC bias.

In Figure 73, one can see that several resonant peaks emerge as the DC bias level is increased. This is expected since the transducer couples significantly more mechanical energy to the system as the bias is increased. If one compares the real impedance of the same cMUT in a water half-space (Figure 70) and a water-filled microchannel (Figure 73), it is evident that additional resonant peaks emerge when the transducer is placed in a channel. These peaks result from thickness resonances in the silicon-water-quartz structure and coupling to guided acoustic waves. The thickness resonances were determined using the analytical model developed for determining the dispersion of 
guided acoustic waves. The frequencies were found to match with the resonances in the impedance data. Unfortunately, the thickness resonances and guided modes occur at nearly identical frequencies and thus make it difficult to discern between the two in the experimental data.

The total power consumed by the system was calculated for bias voltages of 0 and $100 \mathrm{~V}$ and an $\mathrm{AC}$ voltage of $10 \mathrm{Vpp}$. The difference in power represents the power consumed by the transducer since cMUTs couple very little energy to the surrounding medium when no DC bias is applied. The change in power, assuming a 10Vpp AC signal amplitude, is plotted in the following figure.

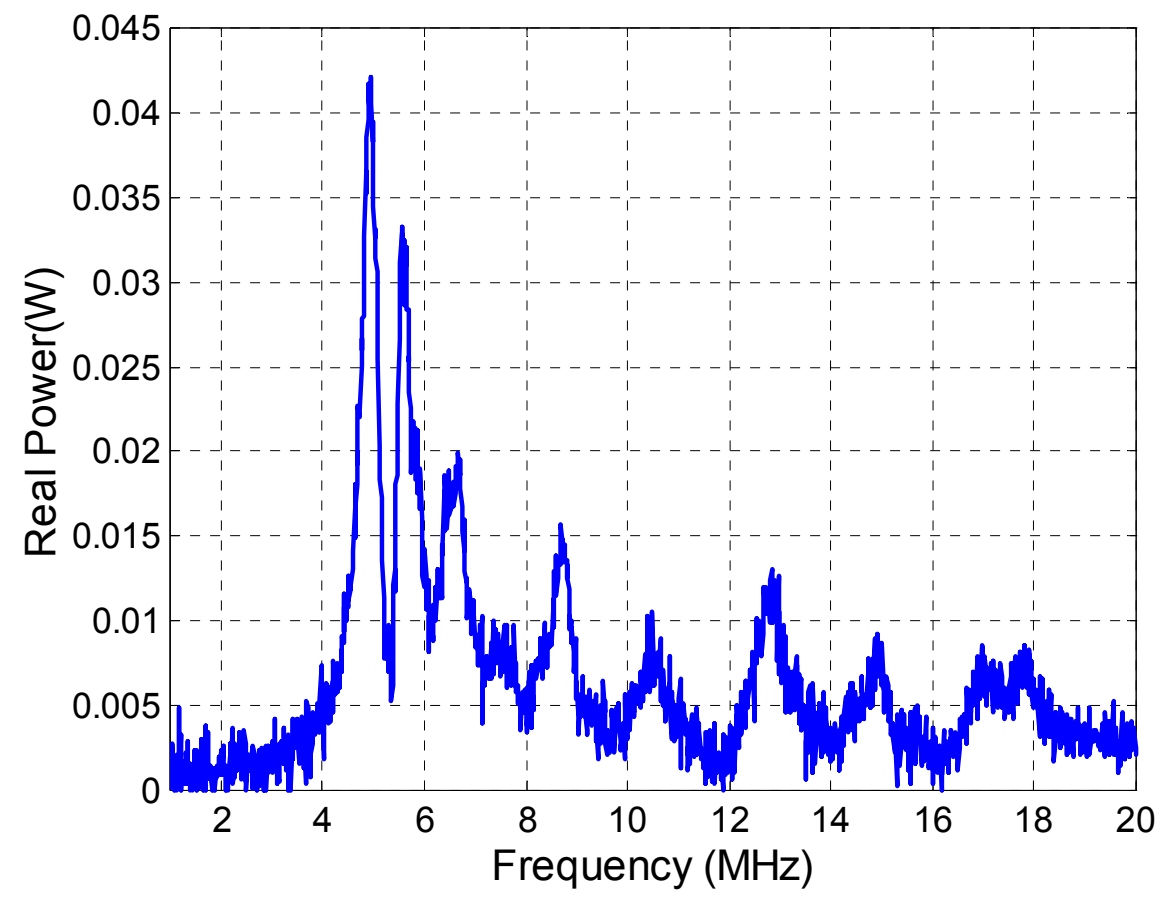

Figure 74Change in real power consumed by an interdigital cMUT in a water-filled, $100 \mu \mathrm{m}$ deep microchannel.

From Figure 74, one can see that the real power consumed by the transducer increases at the resonant frequencies of the system, as expected. 
As previously stated, electrical tuning increases the power consumed by the transducer and ultimately the power delivered to the fluid. Since this tuning is very narrow band, the inductors must be changed for operation at different frequencies. When the same $10 \mathrm{MHz}$ transducer was electrically tuned for a frequency of $10.5 \mathrm{MHz}$, the real power consumed by the transducer increased from $10 \mathrm{~mW}$ to $58 \mathrm{~mW}$, a $7.6 \mathrm{~dB}$ improvement. When the same transducer was tuned to operate at $12.7 \mathrm{MHz}$, the real power consumed by the transducer increased from $12 \mathrm{~mW}$ to $80 \mathrm{~mW}$, an $8.2 \mathrm{~dB}$ improvement. The amount of power consumed by the transducer is of particular importance for characterizing the acoustic streaming based actuator.

To this point the impedance of a single leg of the transducer has been investigated. This measurement does not take into account the effect of a vibrating membrane in the acoustic near field. When the neighboring leg of the transducer is excited, the electrical and mechanical boundary conditions on the leg under test are changed. The altered boundary conditions result in an impedance that is different than the one measured with the standard single leg measurement (Figure 67). To characterize this effect, the impedance of a single leg was measured while the neighboring leg was excited with a phase shifted, continuous wave signal of equal amplitude. The arrangement of equipment is illustrated in Figure 75. 


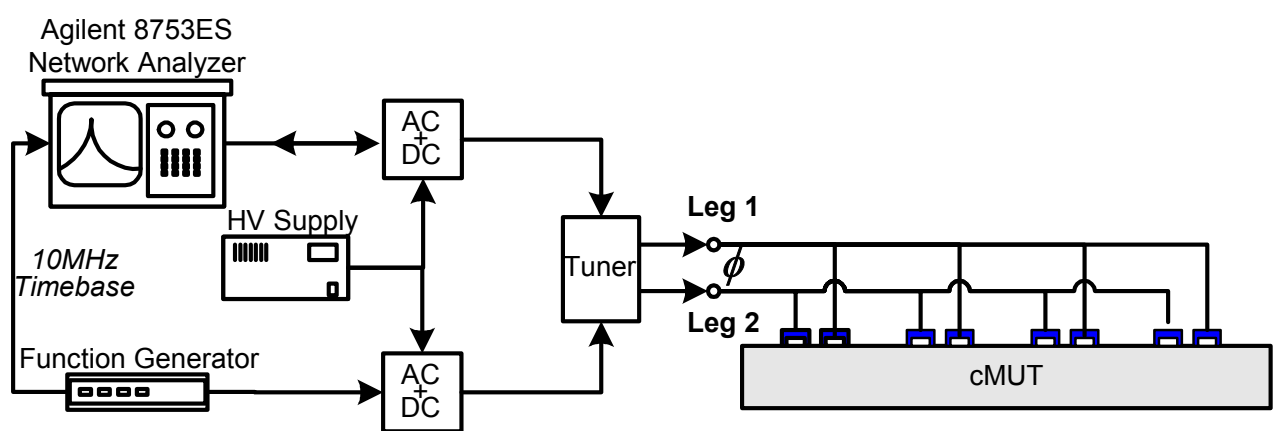

Figure 75 System used to measure the impedance of a single leg of an interdigital cMUT with a phased neighbor.

The output amplitude of the function generator was set equal to the output signal from the network analyzer, which was measured with an oscilloscope. The relative phase between the two inputs was measured after the inductive tuner. The measurement system was controlled with a program written in AgilentVEE to facilitate the phase sweep measurement.

An interdigital cMUT with 10 finger pairs was placed in a water-filled microchannel, $100 \mu \mathrm{m}$ deep and tuned to operate at $10.2 \mathrm{MHz}$. The leg of the transducer connected to the network analyzer was maintained at $0^{\circ}$ while the neighboring leg was excited with phase values ranging from 0 to $360^{\circ}$, as illustrated in Figure 76 . 


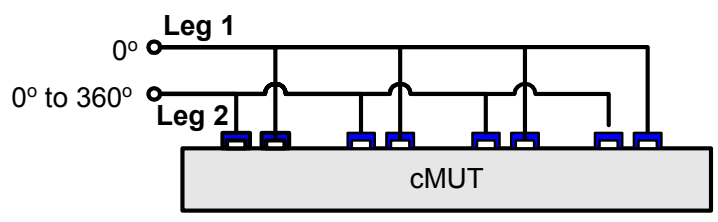

(a)

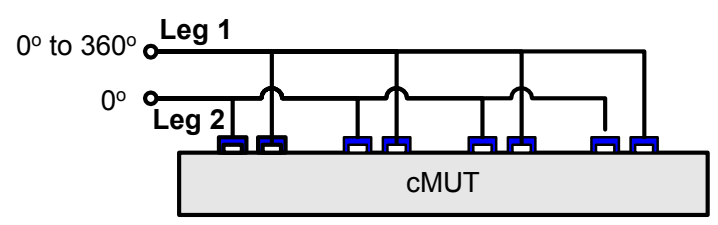

(b)

Figure 76 Schematic of phasing applied to legs of interdigital transducer for measurement of (a) Leg 1 and (b) Leg 2.

The two measurements differ by the sign of the phase angle relative to Leg 1 being fixed at $0^{\circ}$. To properly interpret the measurement data, one must plot the power delivered to the fluid as a function of relative phase, $\phi_{2}-\phi_{1}$.

The impedance measurement was performed at DC bias levels of 0 and $100 \mathrm{~V}$ so that the power consumed by the transducer could be calculated. The change in power as a function of phase for each leg of the transducer is shown in Figure 77. 


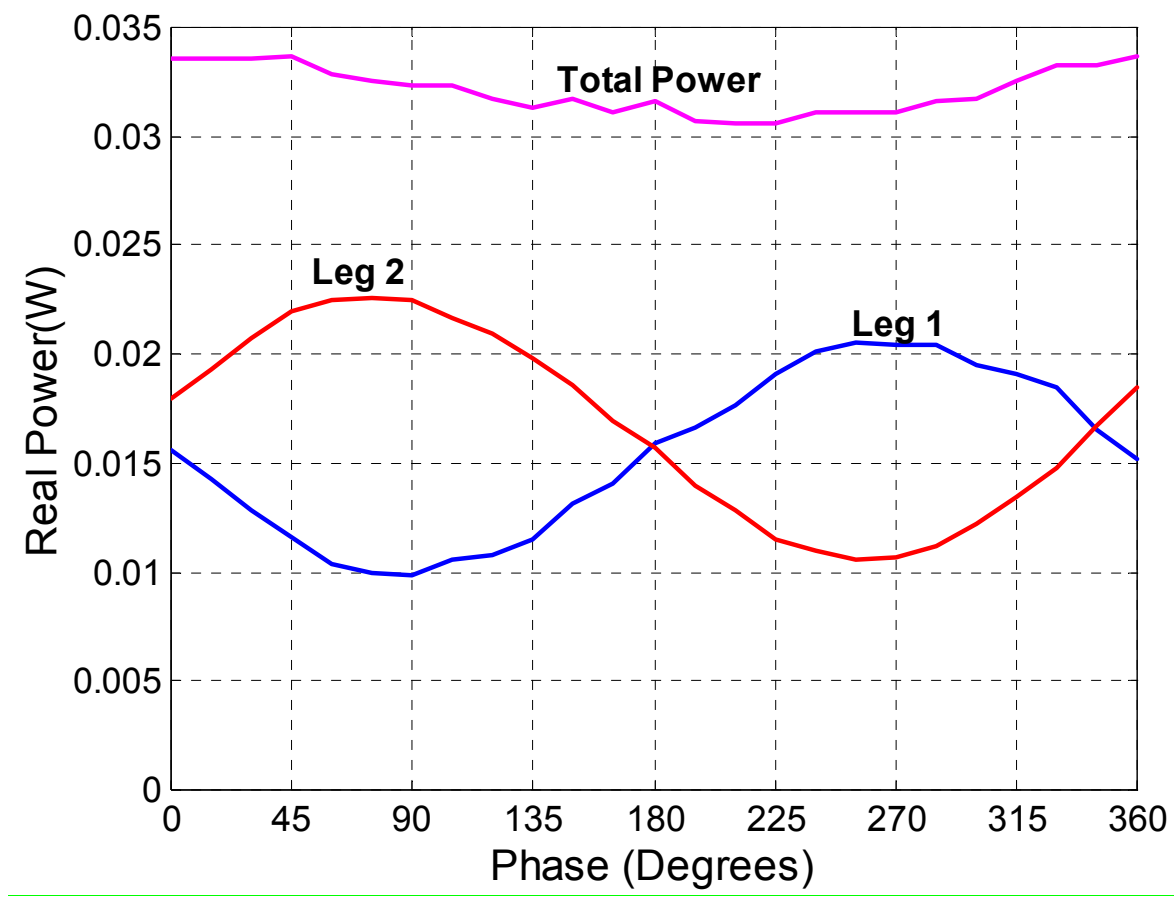

Figure 77 Change in power consumed by a single leg of an interdigital cMUT with a vibrating neighbor.

When an active and phased neighbor is present, the power consumed by the legs of the transducer is a strong function of the relative phase. The amplitude of the data for the two legs is not identical because of differences in electrical tuning. Although the total power is nearly constant with respect to phase, the power delivered to the fluid by a single leg of the transducer is highly dependent on whether the leg is leading or lagging its neighbor in terms of phase. For a phase of $90^{\circ}$, Leg 2 is responsible for delivering the majority of the power to the fluid. Leg 1 is primarily responsible for steering the wave.

The finite element model for an interdigital cMUT in a channel was used to verify that the power delivered to the fluid by a single leg depends on the relative phase. The transducer was simulated for relative phase values ranging from 0 to $360^{\circ}$. The power radiated by each leg was calculated by integrating the intensity over the width of the each 
membrane and summing the results for each leg. The results of this analysis are plotted in Figure 78.

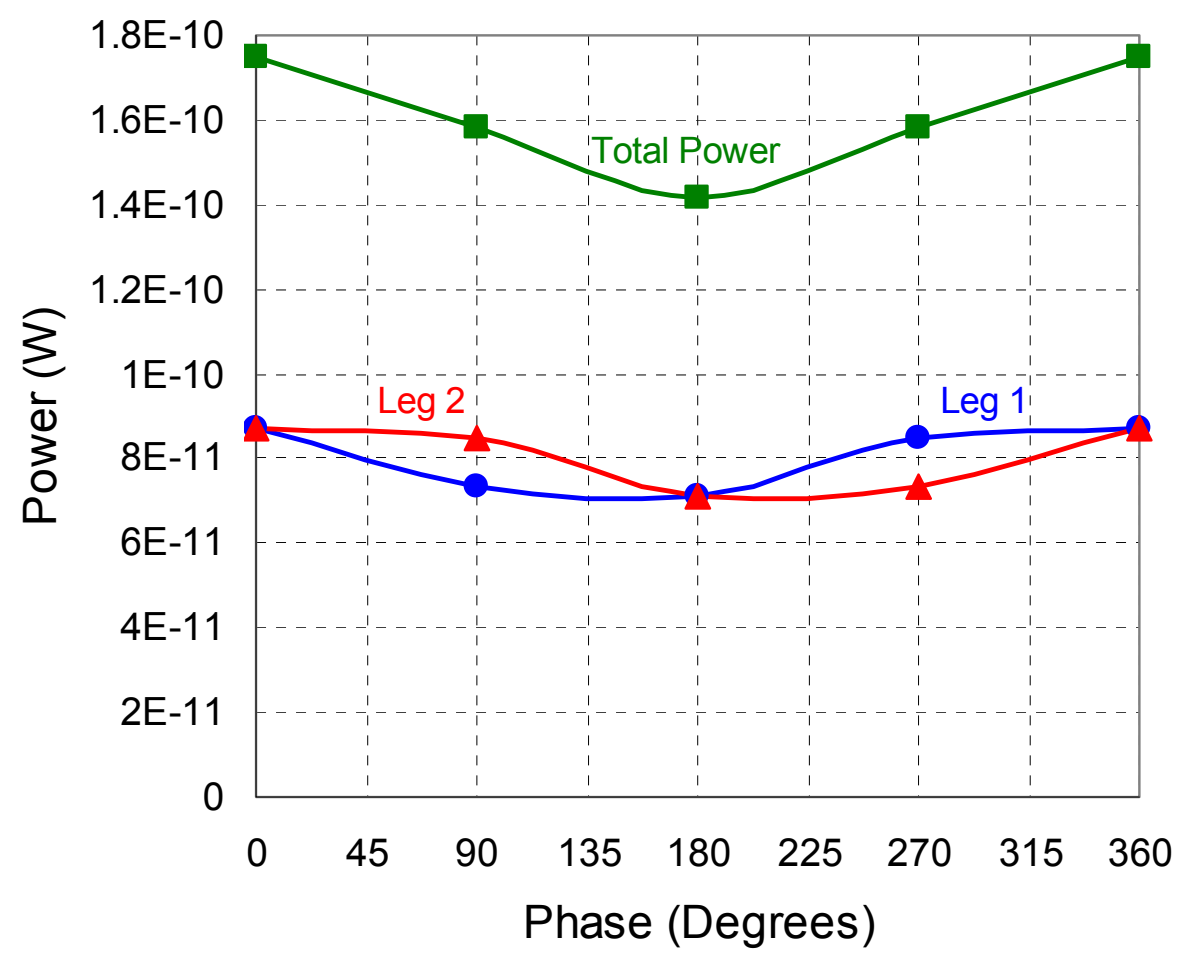

Figure 78 FEM results for power radiated by the individual legs of an interdigital cMUT as a function of phase.

The FEM results confirm that the impedance of the legs of an interdigital transducer depends upon the relative phase between the leg and its neighbor. 


\section{CHAPTER 7}

\section{FLUID SENSING}

Several researchers have developed methods for sensing changes in flow and fluid properties using acoustic transducers. The key benefit of the acoustic sensing technique is that the acoustic waves do not disrupt the flow in the channel. Bradley et al. have shown that flexural plate waves are sensitive to changes in fluid properties and can be used as fluid sensors [65]. As previously stated, these flexural plate wave transducers are difficult to integrate into generic microfluidic systems due to the thin membrane required for operation. Jagannathan et al. have used piezoelectric ultrasonic transducers for performing pulse echo measurements in microchannels, as illustrated in Figure 79 [66].

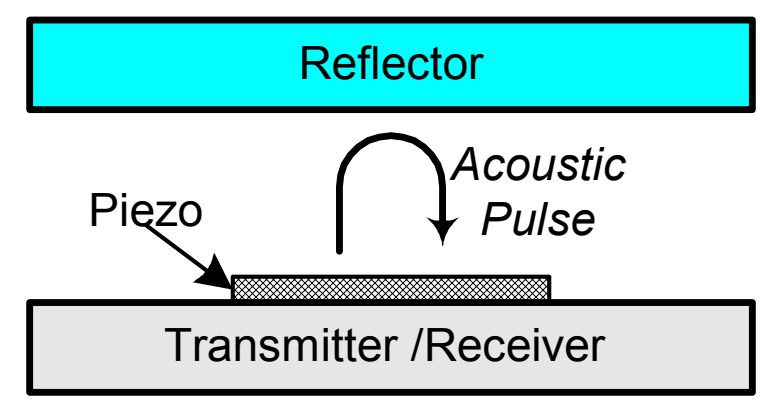

Figure 79 Illustration of pulse-echo measurement used for sensing changes in fluid properties in a microchannel.

The thin-film piezoelectric transducer is excited with a short voltage pulse which in turn generates a pressure pulse in the fluid. This pulse propagates away from the surface of the transducer toward the top of the channel. When it reaches the top of the channel, the acoustic wave is reflected back toward the transducer. The same ultrasonic transducer 
then measures the received signal. The resulting waveform has a pulse-echo response similar to the one shown in Figure 80.

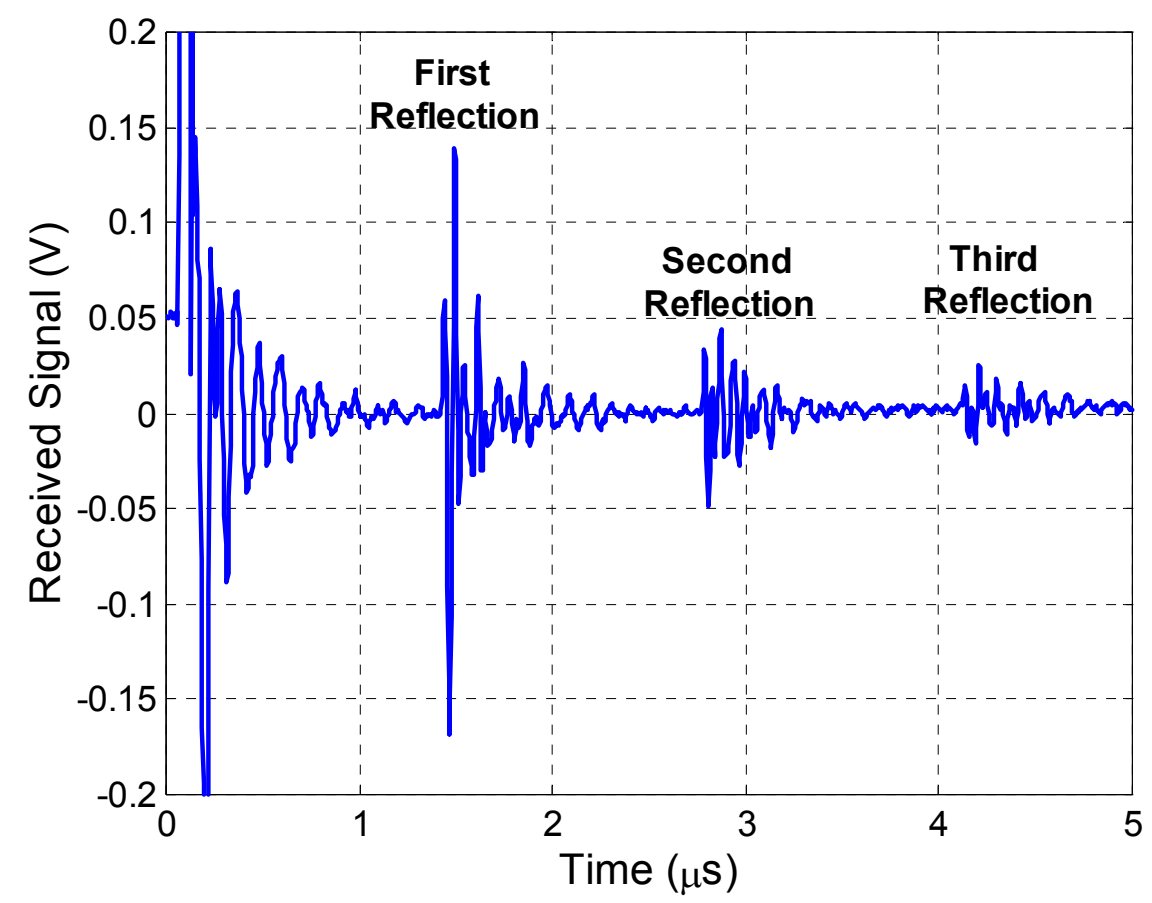

Figure 80 Typical pulse-echo response from an ultrasonic transducer in a microchannel.

The fluid properties, such as sound speed, are determined by monitoring at the arrival time of the first reflection. The problem with this type of measurement stems from the fact that several parameters can affect the arrival time of the signal. Since the sound speed of water is a strong function of temperature, changes in fluid temperature can change the arrival time. A static pressure in the channel can increase the distance between the transducer and the reflector, which also shifts the arrival time of the pulse. Therefore, changes in fluid sound speed and pressure cannot be decoupled. As the channel height is reduced, the arrival time of the reflected pulse decreases and moves the signal into a region dominated by the ringing in the electronics, increasing the difficulty of the 
measurement. In Figure 80, one can see that the signal in the first microsecond is dominated by the ringing of the amplifiers. A reflection at a time of $1 \mu$ s corresponds to a reflector-transducer spacing of $750 \mu \mathrm{m}$, which is quite large for a microfluidic channel. Interdigital cMUTs designed for fluid sensing applications, with a separate transmitter and receiver, have a distinct advantage over the aforementioned approaches: the ability to generate several different acoustic waves with the same transducer. The interdigital cMUT is capable of performing Scholte wave and guided mode transmitreceive measurements, as well pulse-echo measurements. Since the Scholte wave and guided channel modes are sensitive to changes in fluid properties, the received signal can provide information on the visco-elastic properties of the fluid in the channel. The combination of pulse-echo and transmit-receive measurements allows one to decouple the effects of changes in sound speed, pressure, and flow to obtain true measurements of the fluid and flow properties. 


\section{Directionality Measurements}

To demonstrate the Scholte wave transmit-receive measurement and to experimentally verify the results of the finite element models, a 5 finger pair interdigital cMUT was immersed in a water tank simulating a water half-space and excited with a phase shifted, $10 \mathrm{MHz}$ tone burst (Figure 81 ).

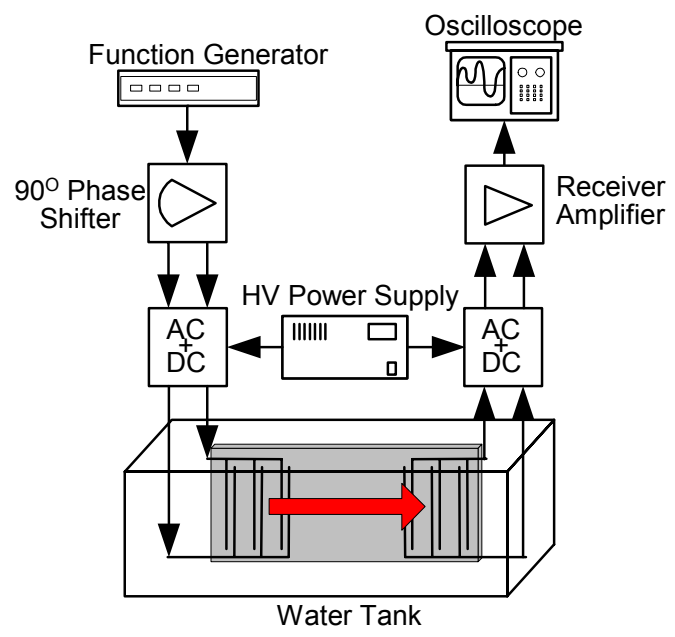

Figure 81 Schematic of experimental setup used for sensing experiments in fluid halfspace.

The $10 \mathrm{MHz}$ tone burst from the function generator was split into two signals with a relative phase difference of $90^{\circ}$ using an operational amplifier based circuit. These two phase shifted, 3Vp-p signals were combined with the $80 \mathrm{~V}$ DC bias voltage before exciting the fingers of the transmitter. A directional Scholte wave was generated and propagated from the transmitter toward the receiver. The output signals from a single leg of the receiver, located $8.9 \mathrm{~mm}$ from the transmitter, are shown in Figure 82 . By changing the phase of the inputs from $+90^{\circ}$ to $-90^{\circ}$, a Scholte wave with $12 \mathrm{~dB}$ of directionality 
was generated, which is in good agreement with the FEM prediction of $10.6 \mathrm{~dB}$. The discrepancy can be attributed to uncertainties in geometry and material properties. From the signals in Figure 82, one can observe the Scholte wave propagating at a velocity slightly less than the speed of sound in the liquid, as expected. The single mode operation makes the interdigital cMUT an attractive liquid sensor since the transmitter and receiver can be connected to form an oscillator [21] or used to perform pitch-catch measurements.

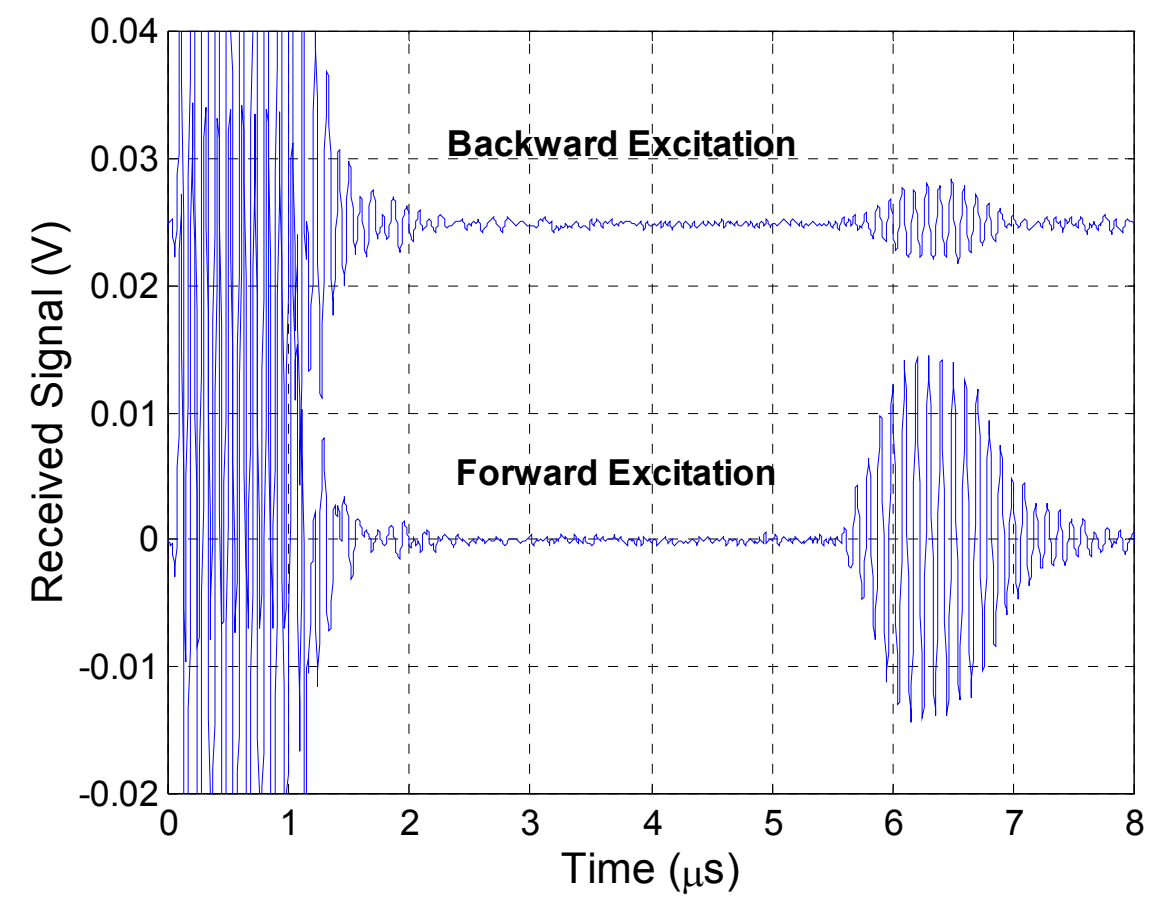

Figure 82 Signals recorded by the cMUT receiver when the transmitter is operated to achieve forward (bottom waveform) and backward (top waveform) excitation at $10 \mathrm{MHz}$ in a water half-space. The transmitter is excited with a 10 cycle tone burst.

To demonstrate the operation of interdigital cMUTs in typical microfluidic structures, a 10 finger pair, $10 \mathrm{MHz}$ interdigital cMUT was placed in a $100 \mu \mathrm{m}$ deep, water-filled channel, using the setup shown in Figure 83. The fingers of the transmitter 
were excited with $3 \mathrm{Vpp}, 10$ cycle tone burst at $10 \mathrm{MHz}$ in addition to $80 \mathrm{~V}$ DC bias. The outputs of the legs of the receiver were recorded independently and phase shifted by $90^{\circ}$ in the computer to obtain maximum Scholte wave amplitude (Figure 84).

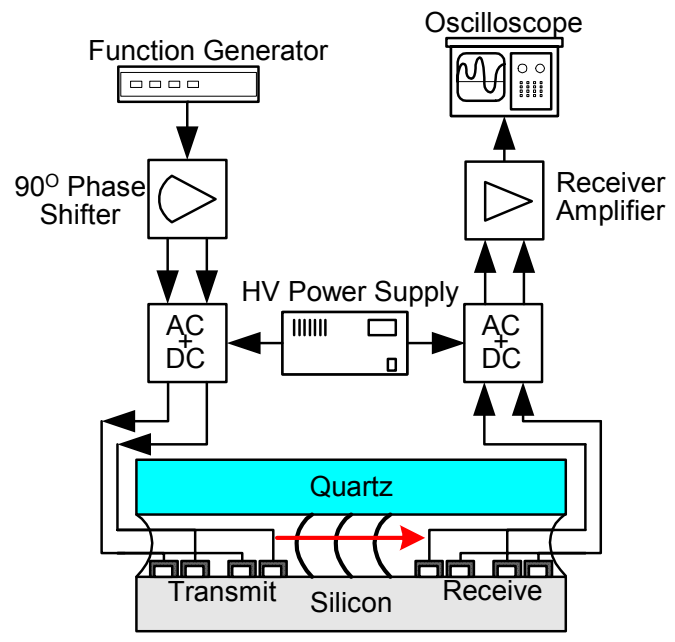

Figure 83 Schematic of experimental setup used for directionality experiments in fluid channel.

In Figure 84, one can see from the received signals that the Scholte wave is the dominant mode propagating in the channel. The directionality was determined to be $13.4 \mathrm{~dB}$, in agreement with the FEM results. The finite element model predicts the increase in directionality with an increased number of transducer fingers. As previously stated, the single mode operation is attractive for liquid sensing applications, and the high directionality and efficiency make pumping feasible. 


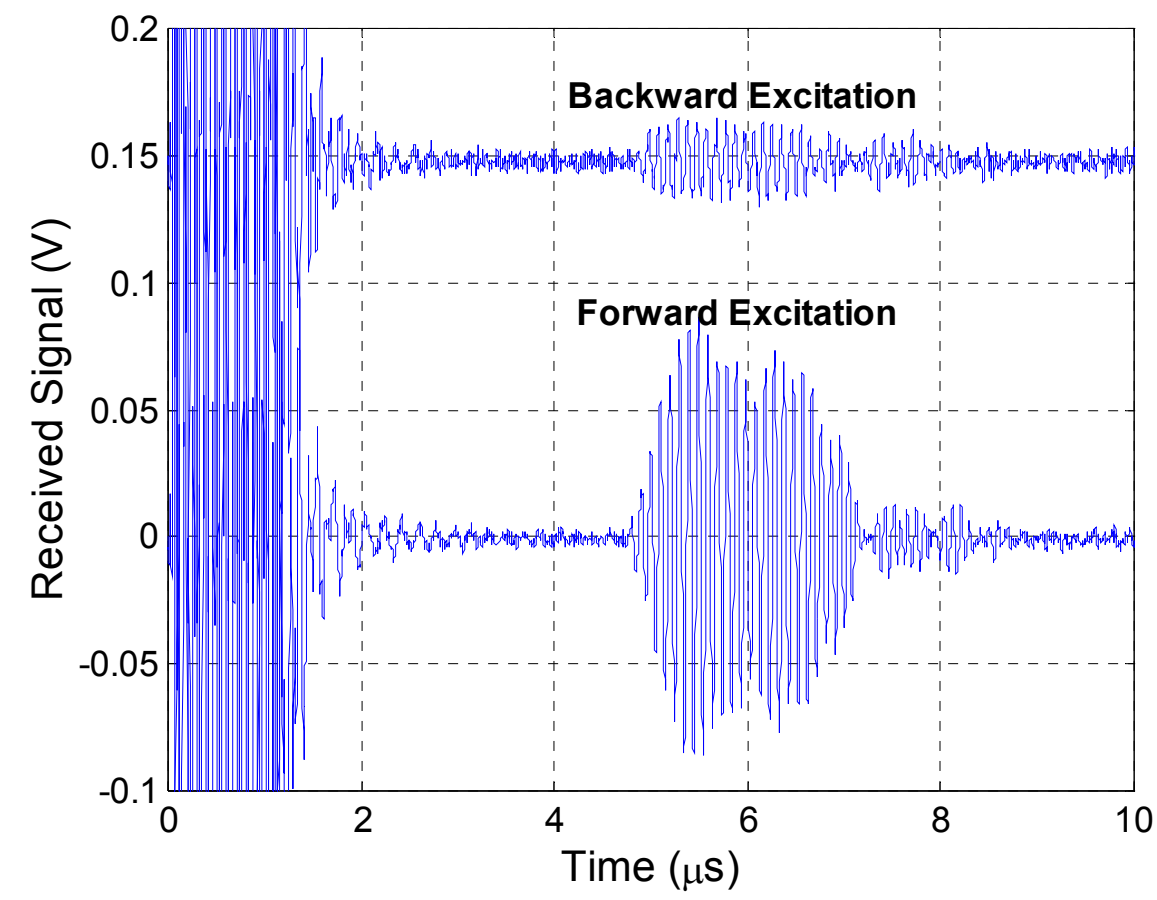

Figure 84 Signals recorded by the cMUT receiver when the transmitter is operated to achieve forward (bottom waveform) and backward (top waveform) excitation at $10 \mathrm{MHz}$ in a water filled channel $100 \mu \mathrm{m}$ deep. The transmitter is excited with a 10 cycle tone burst and is located $6.8 \mathrm{~mm}$ away from the receiver. 


\section{Guided Mode Excitation}

To experimentally verify that guided modes could be selectively excited in a fluid-filled microchannel, an interdigital cMUT designed for operation at $10 \mathrm{MHz}$ was placed in a $100 \mu \mathrm{m}$ deep water-filled channel. The set-up used for the experiments is illustrated in Figure 86.

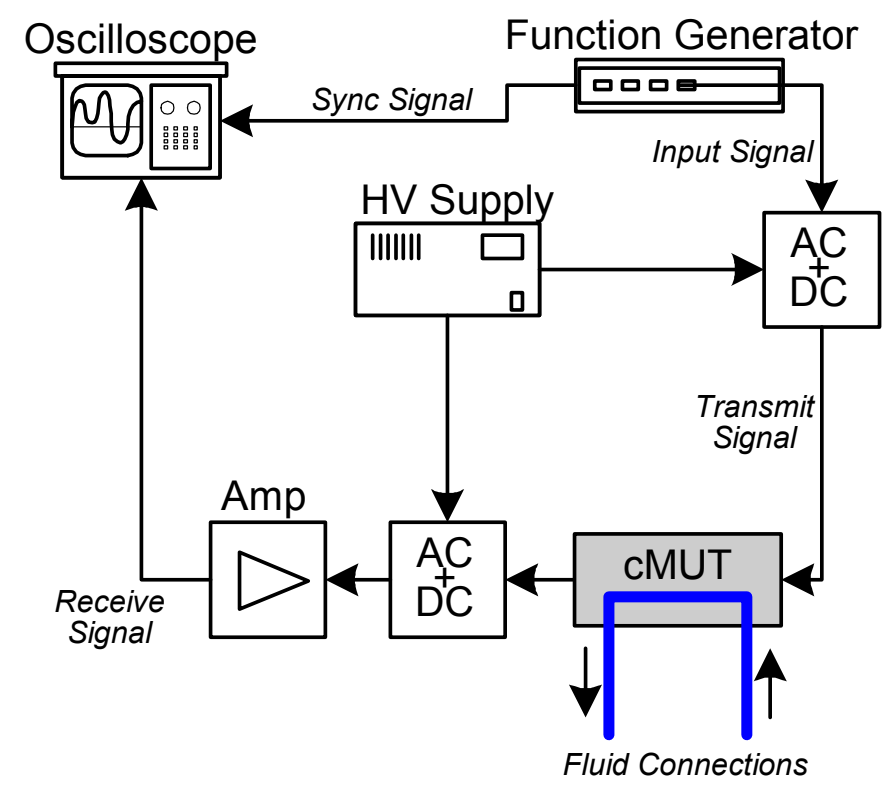

Figure 85 Schematic of experimental set-up used to perform transmit-receive measurements in a fluid-filled microchannel.

The function generator output a $30 \mathrm{~ns}, 10 \mathrm{~V}$ pulse which was added to the $90 \mathrm{~V}$ DC bias. The signal excited the transmitter of the cMUT and generated an acoustic pulse which propagated along the channel toward the receiver, as illustrated in Figure 86. The receiver was also biased, and the received signal was measured and recorded using an oscilloscope. 


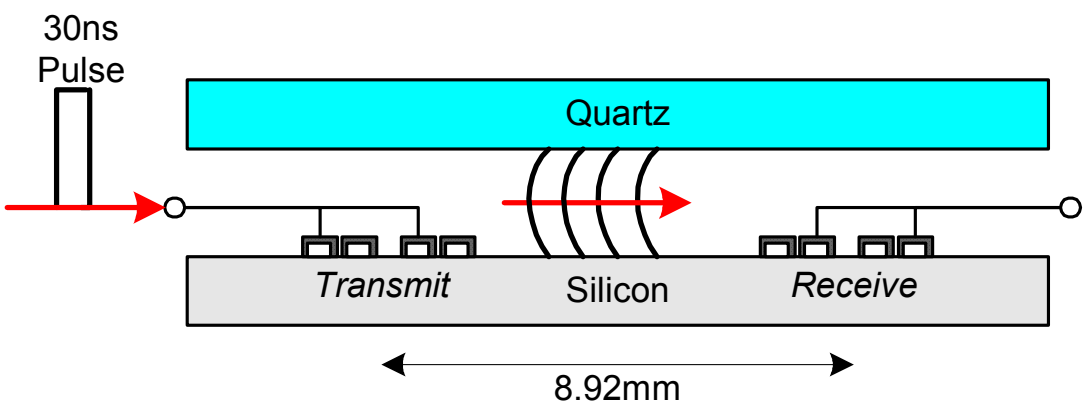

Figure 86 Illustration of guided mode propagation resulting from impulse excitation of an interdigital cMUT in a $100 \mu \mathrm{m}$ water filled microchannel.

The pulse generated guided waves at many frequencies, but only certain modes were able to propagate down the channel and reach the receiver. The Fourier transform of the received signal at the receiver was plotted against phase velocity and illustrates the modes that can propagate in the channel (Figure 87). In the plot, one can see that there are significant peaks at $5.25,6.78,10,12.7$ and $17.4 \mathrm{MHz}$. The peaks at 5.25 and $6.78 \mathrm{MHz}$ correspond to a thickness resonance in the silicon-water-quartz system and the damped resonant frequency of the membrane, respectively. The other small peaks in the impulse response correspond to thickness resonances of the silicon plate-water-quartz plate structure. 


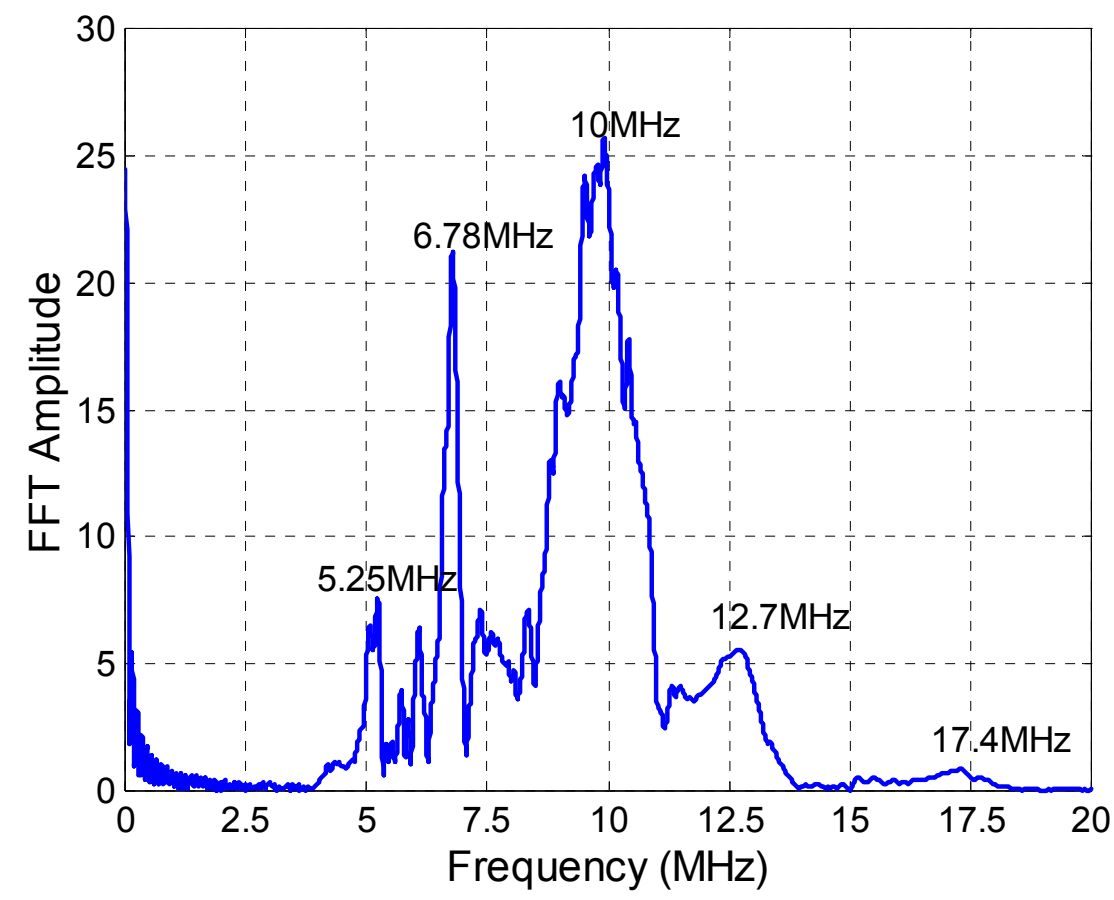

Figure 87 Impulse response of transmit-receive interdigital cMUT in a100 $\mu \mathrm{m}$ water-filled microchannel.

To determine the origin of the other resonances with frequencies of $10,12.7$, and 17.4MHz, the analytical model was used to determine the dispersion curve for the $100 \mu \mathrm{m}$ channel as a function of frequency. Then, the phase velocities of the modes that could be exicted by the interdigital structure with a periodicity of $146 \mu \mathrm{m}$ were calculated using

$$
V_{\text {Phase,Mode }}=\lambda_{\text {Transducer }} \cdot f_{\text {excitation }} \text {. }
$$

Here $V_{\text {Phase,Mode }}$ is the phase velocity of a mode that has a wavelength that matches the periodicity of the transducer, $\lambda_{\text {Transducer, }}$, at a particular frequency, $f_{\text {excitation, }}$. The frequency of excitation was varied from 1 to $20 \mathrm{MHz}$ and plotted on top of the dispersion curve as shown in Figure 88. 


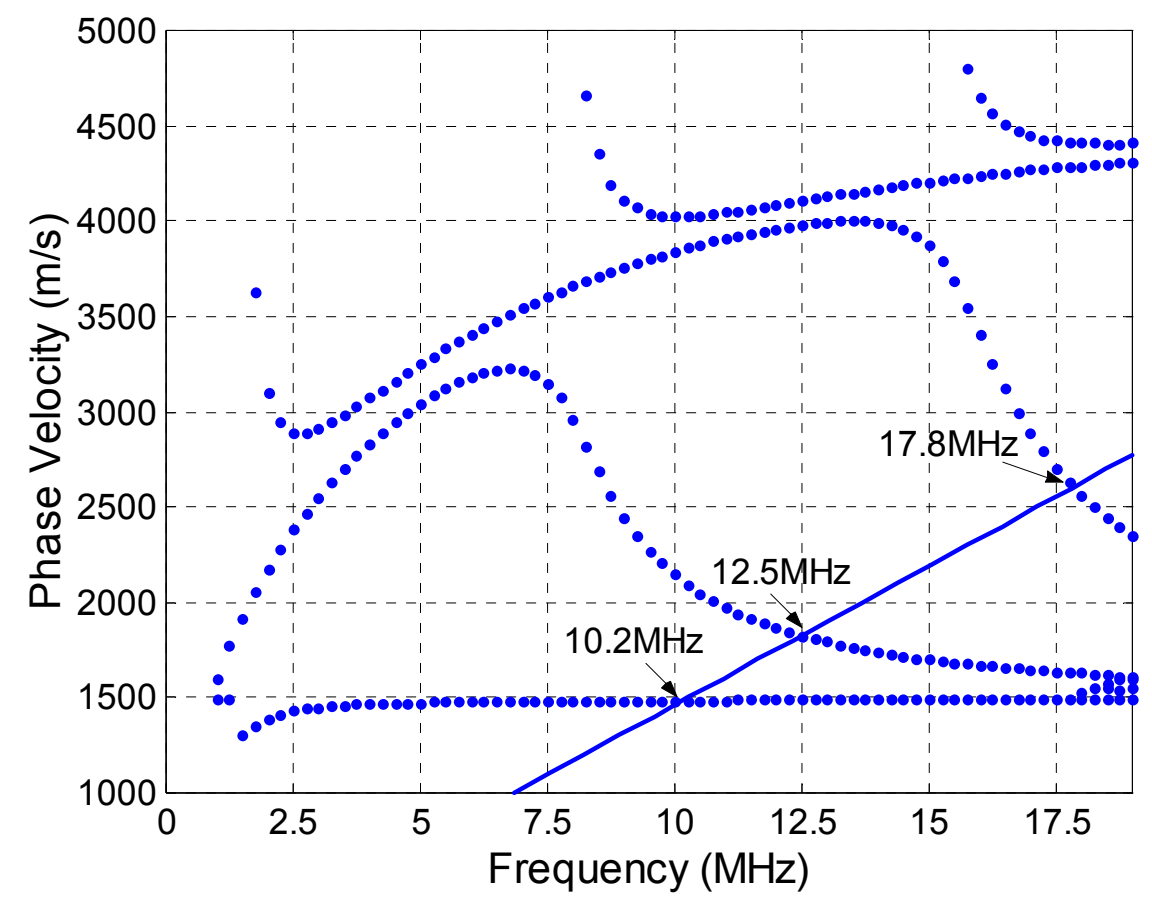

Figure 88 Dispersion curve illustrating the propagating modes in a $100 \mu \mathrm{m}$ water-filled channel.

The solid line represents the phase velocity-frequency combinations that match the periodicity of the interdigital cMUT. The numbers on the figure indicate the frequency at which the intersection occurs. The intersections of the solid line and the dotted lines represent the modes that can be excited and propagate in this particular channel. When the results are compared to the impulse response for the channel, one sees that the frequencies match quite well. The analytical model predicts that modes with frequencies of $10.2,12.5$, and $17.8 \mathrm{MHz}$ can propagate, while the experimental data shows that modes with frequencies of $10,12.7$, and $17.4 \mathrm{MHz}$ actually propagate. The small discrepancy in frequencies can be attributed to variations in the channel height since the guided channel modes are very sensitive to changes in channel height, as shown by the analytical model. 
To verify that the various guided modes could be selectively generated, the interdigital cMUT was excited with tone bursts at the frequencies corresponding to the Scholte wave and guided modes. The cMUTs were biased to $90 \mathrm{~V}$ and excited with a $10 \mathrm{Vpp}, 20 \mathrm{cycle}$ tone burst at the frequency of interest. The resulting waveforms for frequencies of 10, 12.7 and 17.4 MHz are shown in Figure 89.

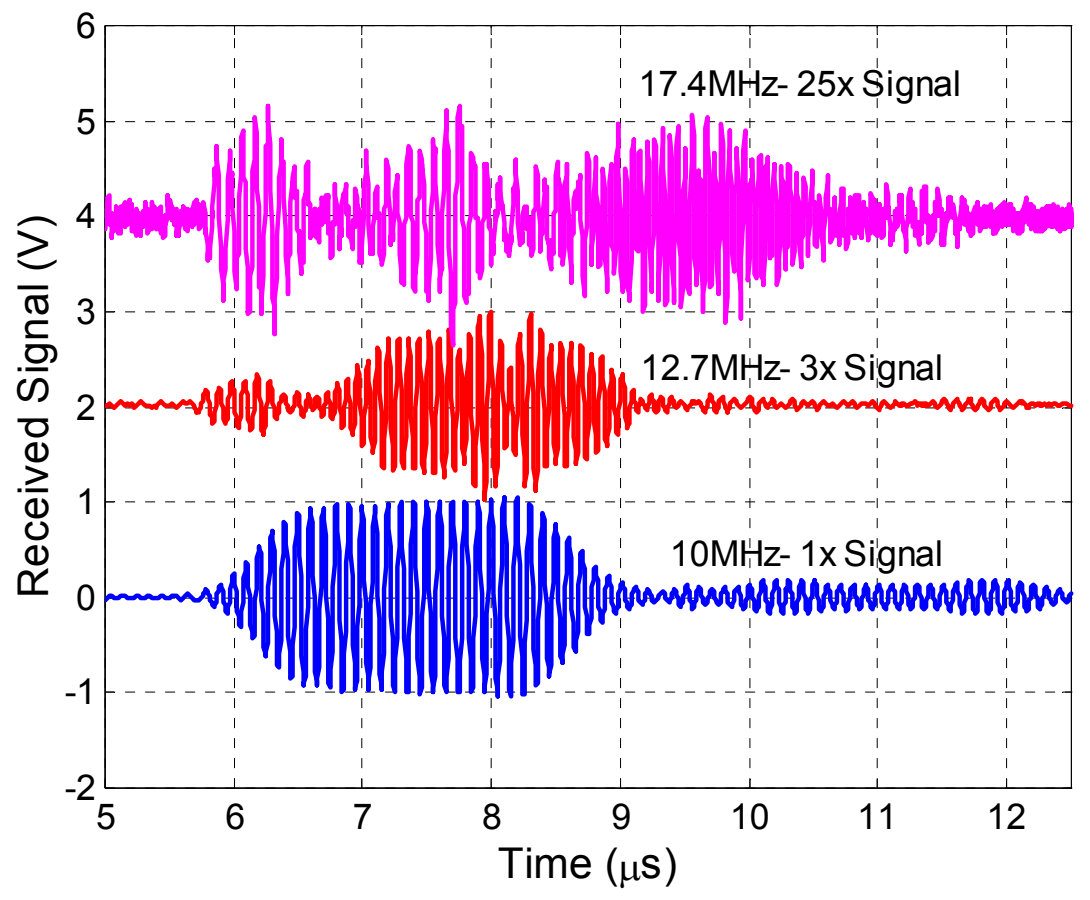

Figure 89 Received signals for guided modes excited at 10,12.7 and 17.4 MHz. Note that the signals for 12.7 and $17.4 \mathrm{MHz}$ have been scaled by factors of 3 and 25 , respectively.

In Figure 89, one can see that the first arrival time occurs at approximately $5.8 \mu \mathrm{s}$. This corresponds to the Scholte wave which is preferentially generated by this transducer structure. Since the transducer is excited by a tone burst, there are other frequencies contained in the signal. The frequency components at $10 \mathrm{MHz}$ couple well to the structure, and thus the Scholte wave is excited in all cases. 
In Figure 89, one can see that different modes propagate when the frequency of excitation is changed to match the guided mode frequency. The analytical model was used to predict the group velocities, and thus arrival times, of these guided modes. The results of this analysis are summarized in Table 7.

Table 7 Summary of phase velocity, group velocity and arrival time information for guided modes propagating in a $100 \mu \mathrm{m}$ water-filled channel.

\begin{tabular}{|c|c|c|c|}
\hline Mode & $\begin{array}{c}\text { Phase Velocity } \\
(\mathbf{m} / \mathbf{s})\end{array}$ & $\begin{array}{c}\text { Group Velocity } \\
(\mathbf{m} / \mathbf{s})\end{array}$ & $\begin{array}{c}\text { Theoretical Arrival } \\
\text { Time }(\boldsymbol{\mu s})\end{array}$ \\
\hline Scholte: $10 \mathrm{MHz}$ & 1486 & 1496.9 & 5.96 \\
\hline Guided 1: $12.7 \mathrm{MHz}$ & 1812 & 1160 & 7.7 \\
\hline Guided 2: $17.4 \mathrm{MHz}$ & 2738 & 866.2 & 10.3 \\
\hline
\end{tabular}

The predicted arrival times agree to a reasonable extent with the experimental data. Again, the discrepancy can be attributed to the highly dispersive nature of the guided waves with respect to channel height. 


\section{$\underline{\text { Zero-Crossing Measurements }}$}

After establishing that Scholte waves and guided modes could be generated in microfluidic channels, the sensitivity of the modes to changes in fluid properties was investigated. The analytical model indicates that both the group and phase velocity of each mode is sensitive to changes in fluid properties. However, it is difficult to accurately measure the group velocity since the arrival time of the received signal is difficult to discern. This is because several modes could be propagating at one time and the resulting interference complicates the received signal. Therefore, the phase velocity was used to measure the change in fluid properties.

The sensitivity of the phase velocity of the mode of interest to changes in fluid properties was determined by monitoring the location of a single zero-crossing of the

received signal. As the fluid properties are changed, the zero crossing shifts in time as a result of a change in phase velocity as shown in Figure 90. 


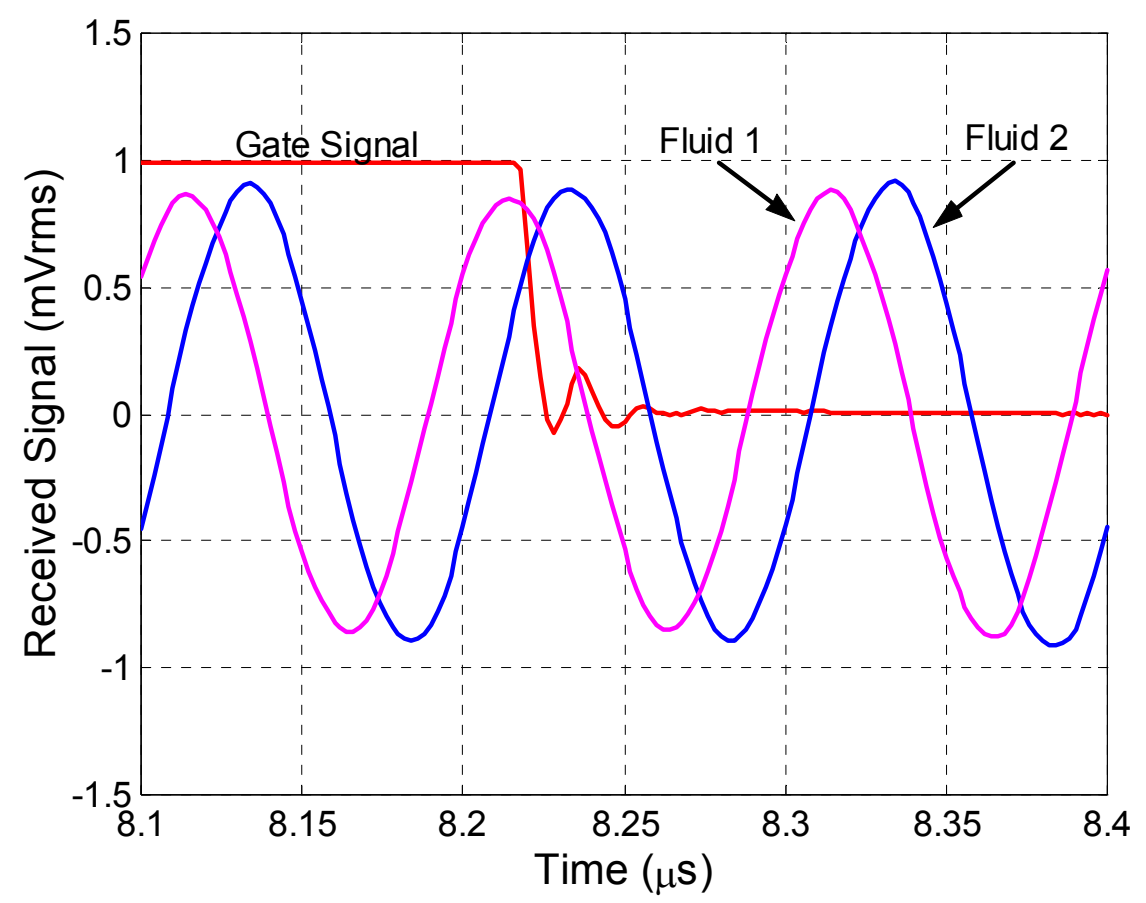

Figure 90 Experimental data illustrating the change in phase velocity, and thus location of the zero crossing, resulting from a change in fluid properties. The gate signal tells the time interval counter which zero crossing to monitor.

The system illustrated in Figure 91 was used to generate a 25 cycle tone burst at the frequency of interest. This time-harmonic signal was combined with the DC bias voltage before exciting the transmitter. The gate signal from the pulse generator was used to inform the time interval counter (TIC) which zero crossing to monitor, as shown in Figure 90. The TIC was set to monitor the first zero crossing with positive slope after the gating signal. For large changes in fluid properties, it was necessary to adjust the width of the gating signal to ensure that the same zero crossing was being monitored. The limitation of measurement accuracy for the system was the jitter, or noise, in the measurement on the TIC. 


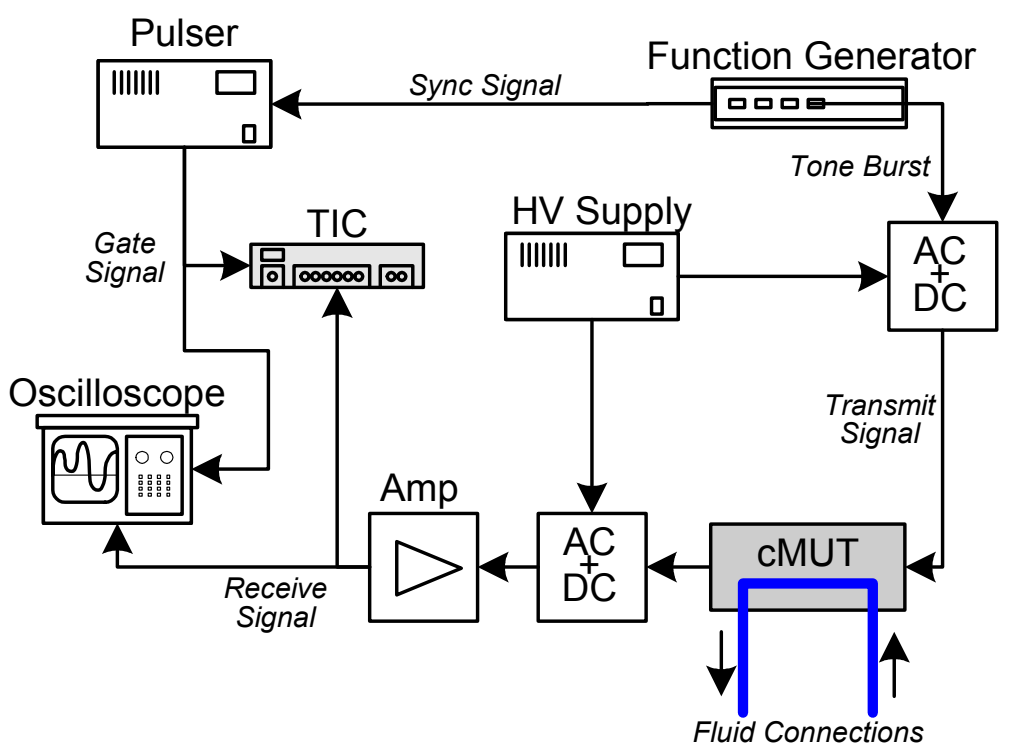

(a)

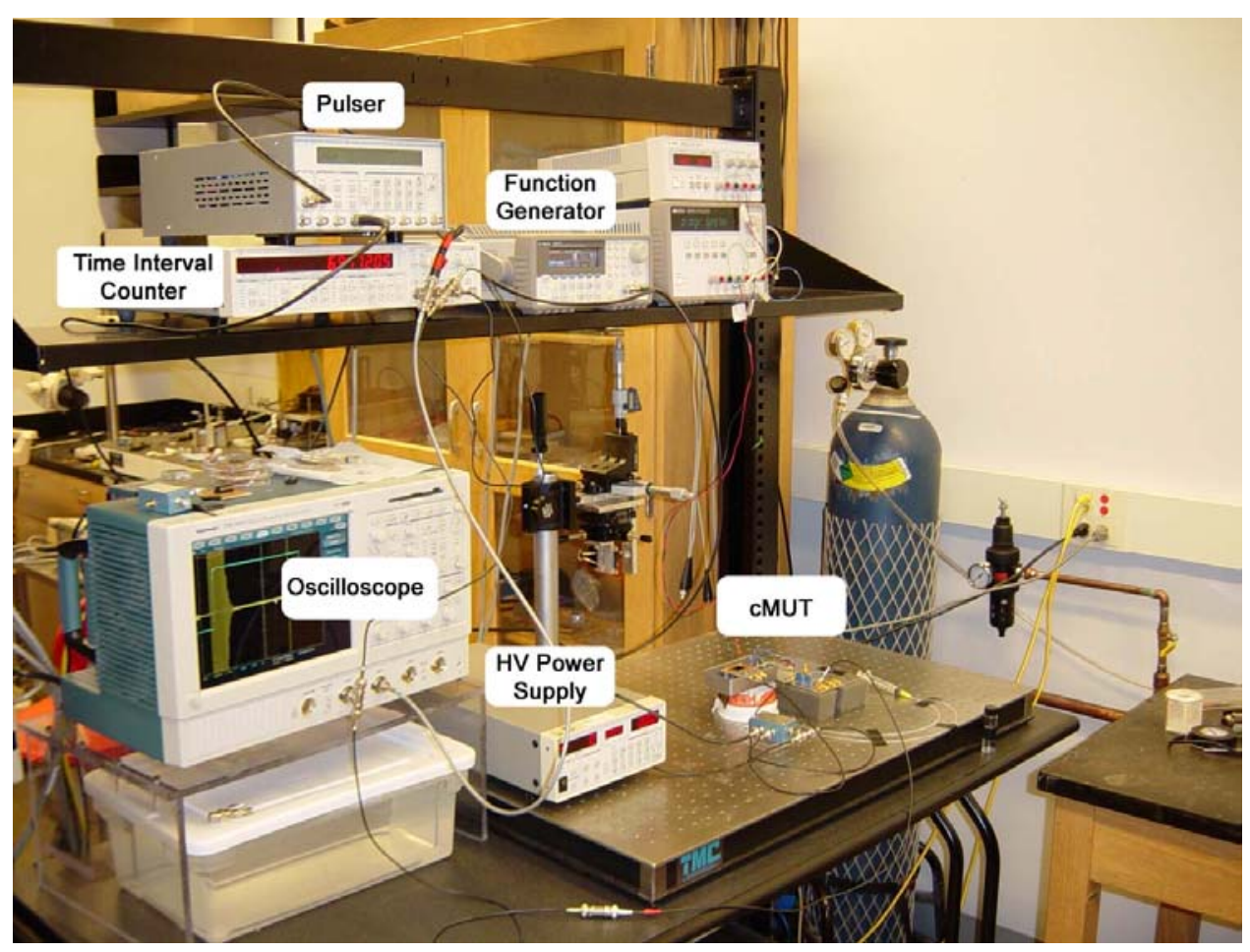

(b)

Figure 91 (a) Illustration of system used to perform zero-crossing measurements and (b) photograph of actual experimental set-up. 
The DC bias voltage was varied for a fixed AC voltage of 5Vpp to determine the effect of bias voltage on the received signal amplitude. The amplitude of the received signal increased with bias voltage as expected (Figure 92).

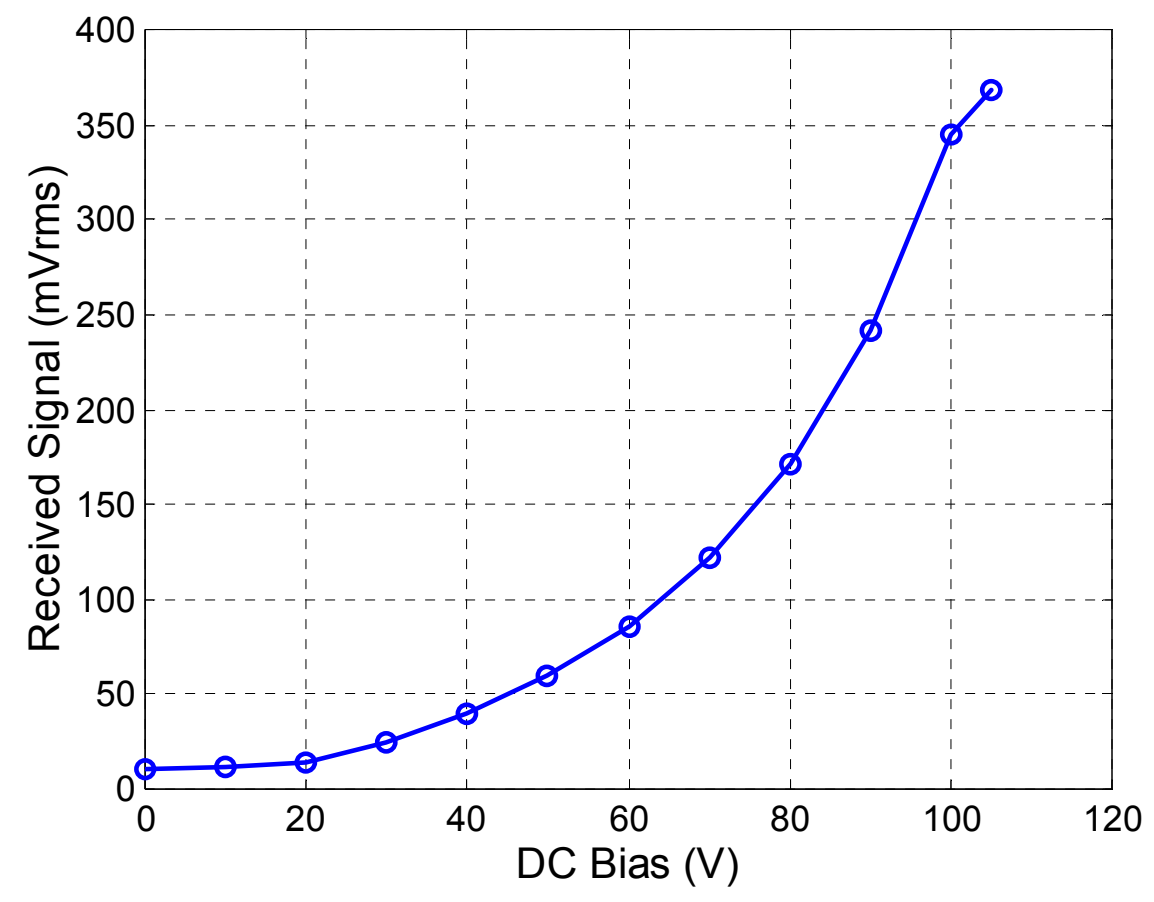

Figure 92 Plot of the received signal amplitude as a function of DC bias.

The amplitude of the received signal continued to increase until it reached a bias voltage of $100 \mathrm{~V}$. This voltage was confirmed to be the collapse voltage of the device and explains why the amplitude began to decrease. Once the membrane reached its collapse voltage, it remained collapsed and the AC signal resulted in a smaller overall membrane displacement amplitude. This in turn began to decrease the amplitude of the transmitted and received signals.

The signal-to-noise ratio (SNR) was determined for a fixed DC bias as a function of AC signal amplitude from the following 


$$
S N R=20 \cdot \log _{10}\left(\frac{V_{r m s, \text { Signal }}}{V_{\text {rms Noise }}}\right)
$$

where $V_{r m s, \text { Signal }}$ is the root mean square (RMS) voltage of the received signal and $V_{r m s}$ Noise is the RMS voltage of the noise. The results of an experiment in which the AC voltage was varied for a fixed DC bias of $90 \mathrm{~V}$ is shown in Figure 93.

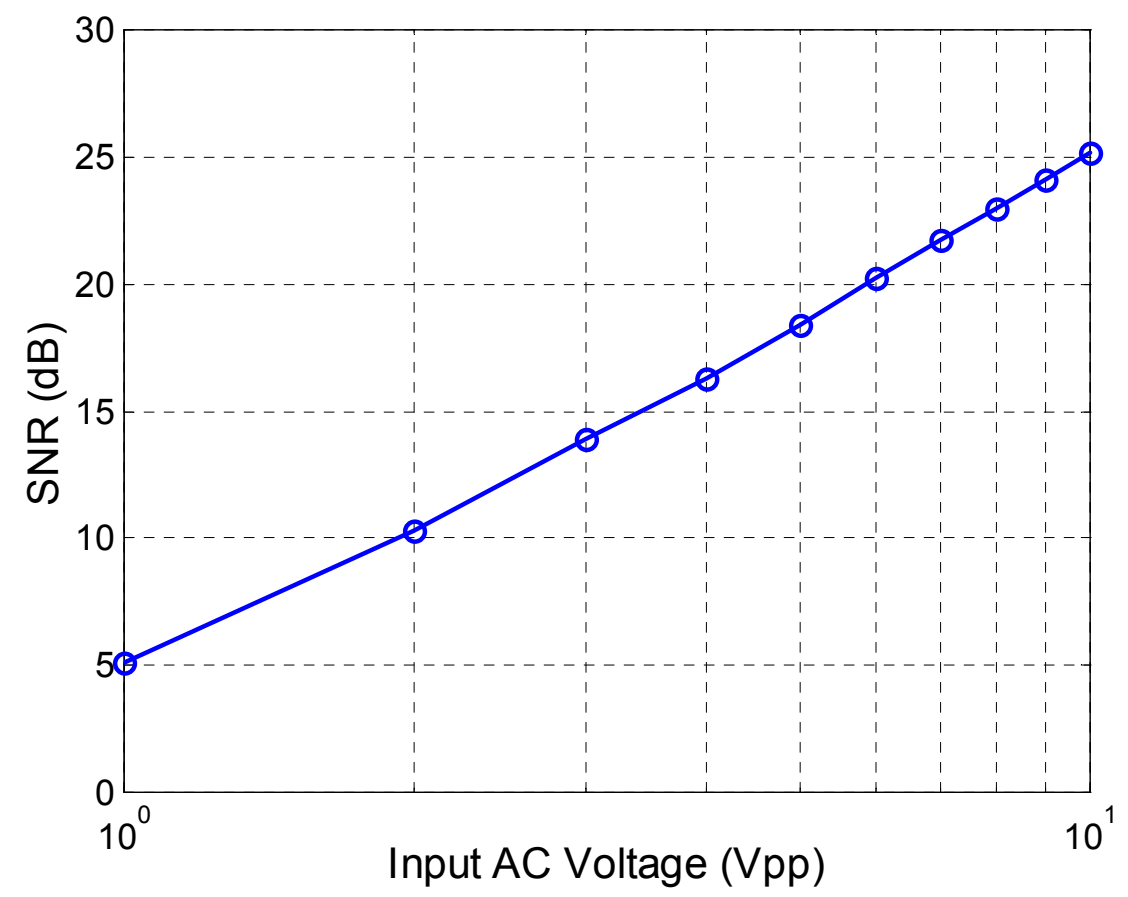

Figure 93 Plot of the signal-to-noise ratio as a function of input AC voltage.

From the plot, one can see that the SNR increases linearly with AC voltage when plotted on a logarithmic scale. This is expected since SNR increases with the power in the received signal. The maximum SNR obtained with the system was $25 \mathrm{~dB}$.

The jitter in the time interval measurement was found to decrease as the SNR increased, as shown in Figure 94. 


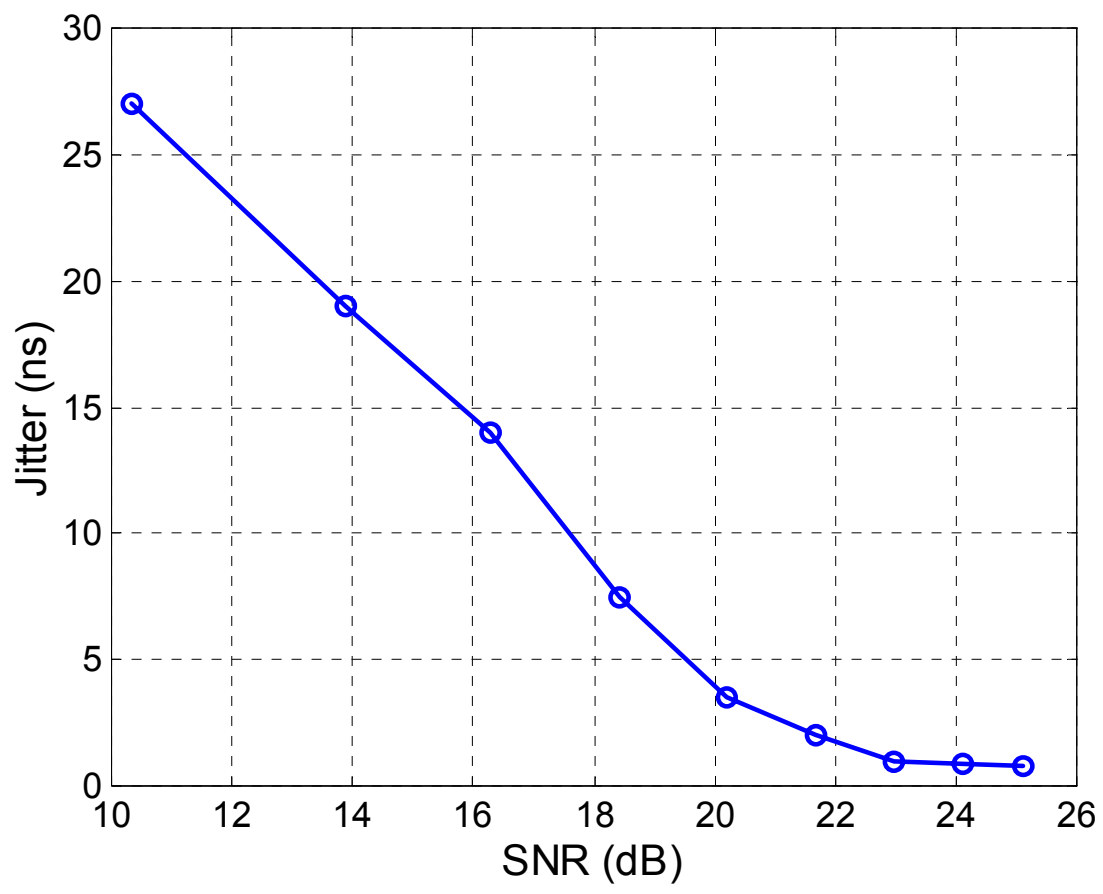

Figure 94 Jitter in the time interval measurement as a function of the SNR.

The minimum jitter in the measurement was approximately $1 \mathrm{~ns}$, which establishes the accuracy of the system. For a velocity measurement, a jitter of 1 ns corresponds to a change in sound speed of $0.25 \mathrm{~m} / \mathrm{s}$ for a distance between transmitter and receiver of $8.92 \mathrm{~mm}$. The jitter can be further reduced by increasing the amplitude of the input signal to increase the SNR and by improving the electrical shielding. 


\section{Sensitivity to Changes in Fluid Sound Speed}

After characterizing the measurement system, the ability to sense changes in fluid properties was investigated. Mixtures of varying amounts of glycerol in water were used for the experiments for several reasons. First, glycerol is miscible in water which results in homogenous mixtures with stable properties. Second, the sound speed, density, and viscosity of glycerol-water mixtures are well established in the literature and can thus be used to calibrate the system. Although the temperature of the fluid could have been varied to study the velocity-based measurement system, water-glycerol mixtures have stable properties and simplify the experiments.

The properties of water-glycerol mixtures at $20^{\circ} \mathrm{C}$ are presented in Table 8 . The density and sound speed data were obtained from a linear interpolation between the values for pure water and pure glycerol. The viscosity information comes from published values [67].

Table 8 Properties of water-glycerol mixtures at $20^{\circ} \mathrm{C}$.

\begin{tabular}{|c|c|c|c|}
\hline $\begin{array}{c}\text { Wt \% Glycerol } \\
\text { in Water }\end{array}$ & $\begin{array}{c}\text { Viscosity } \\
\text { (Pa-s) }\end{array}$ & $\begin{array}{c}\text { Density } \\
\mathbf{( k g / \mathbf { m } ^ { \mathbf { 3 } } )}\end{array}$ & $\begin{array}{c}\text { Sound Speed } \\
\mathbf{( m / s )}\end{array}$ \\
\hline 0 & $9.14 \mathrm{E}-04$ & 997 & 1497 \\
\hline 24 & $1.80 \mathrm{E}-03$ & 1056.1 & 1595 \\
\hline 44 & $3.92 \mathrm{E}-03$ & 1109.2 & 1676 \\
\hline 56 & $7.24 \mathrm{E}-03$ & 1141.9 & 1725 \\
\hline 64 & $1.21 \mathrm{E}-02$ & 1164.3 & 1757 \\
\hline 72 & $2.24 \mathrm{E}-02$ & 1186.6 & 1790 \\
\hline 80 & $4.87 \mathrm{E}-02$ & 1208.5 & 1823 \\
\hline 84 & $7.73 \mathrm{E}-02$ & 1219.2 & 1839 \\
\hline 88 & $1.31 \mathrm{E}-01$ & 1229.9 & 1855 \\
\hline 92 & $2.40 \mathrm{E}-01$ & 1240.4 & 1871 \\
\hline 96 & $4.72 \mathrm{E}-01$ & 1250.8 & 1888 \\
\hline 100 & $1.04 \mathrm{E}+00$ & 1261.1 & 1904 \\
\hline
\end{tabular}


Transmit-receive measurements were made in a $100 \mu \mathrm{m}$ deep microchannel using a cMUT designed for operation at $10 \mathrm{MHz}$. The DC bias and AC voltage were held constant at $90 \mathrm{~V}$ and $10 \mathrm{Vpp}$, respectively. The concentration of glycerol in water was slowly varied from 0 to $20 \mathrm{wt} \%$. For each incremental change in glycerol concentration, the shift in the zero-crossing was recorded. The experiment was performed using a $25 \mathrm{cycle}$ tone burst at $10 \mathrm{MHz}$ for the Scholte wave and at $12.7 \mathrm{MHz}$ for the first guided mode. The measured time shift was converted to a phase change to remove the frequency dependence using

$$
\Delta \phi=\frac{\Delta t}{T} \cdot 360^{\circ}
$$

where $\Delta \phi$ is the change in phase, $\Delta t$ is the measured time shift, and $T$ is the period of one cycle of a sine wave at the excitation frequency.

The change in phase of the Scholte wave is plotted in Figure 95 as a function of the concentration of glycerol in water. The solid line represents the theoretical change in phase for a plane wave propagating in an infinite medium with the properties of the water-glycerol mixture. The dashed line represents a linear curve fit of the experimentally obtained data for the change in phase of the Scholte wave. 


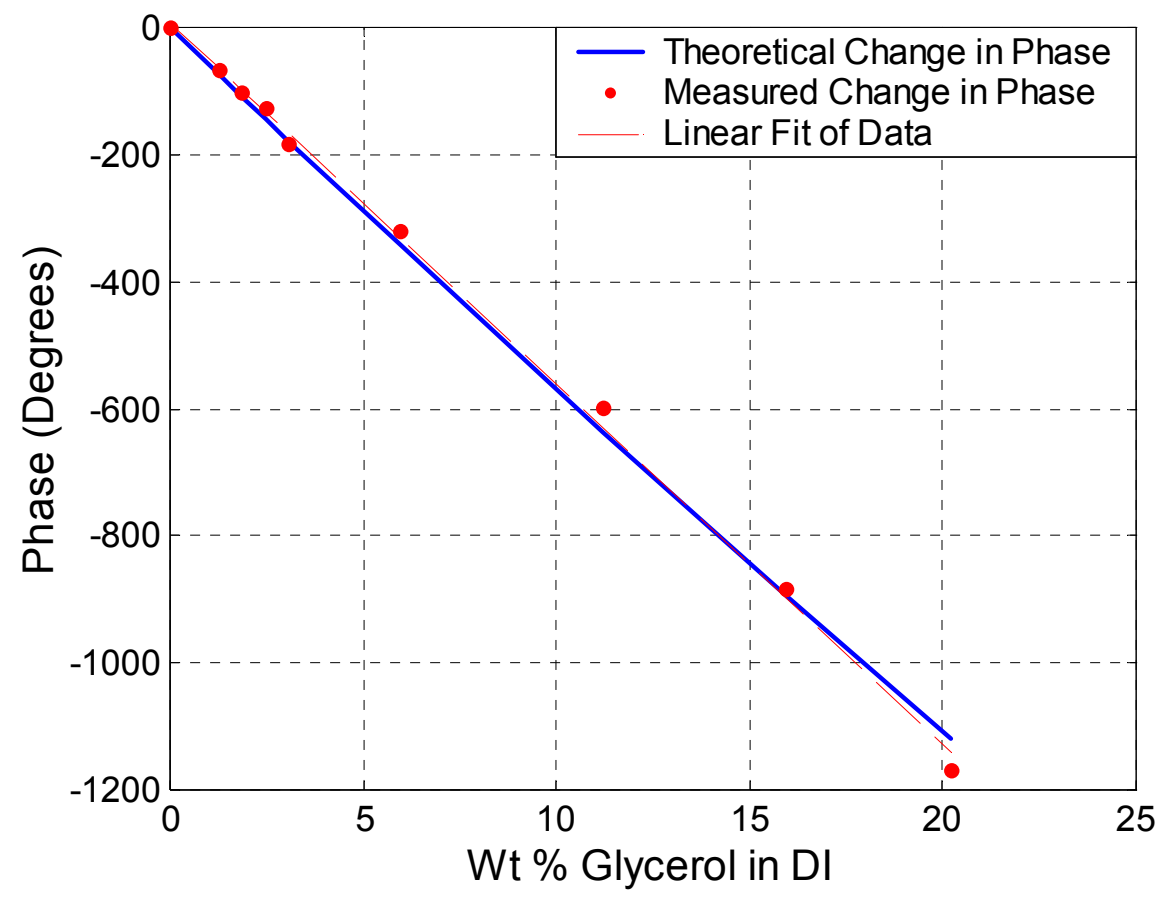

Figure 95 Change in phase of the received Scholte wave resulting from a change in the concentration of glycerol in de-ionized water.

From the plot, one can see that the change in phase for the Scholte wave is nearly identical to the change in phase for a bulk pressure wave in the fluid. The sensitivity of the Scholte wave to changes in the sound speed of the fluid was determined by fitting a line to the experimental data and comparing it with the line representing the theoretical change in phase. The ratio of the slopes of these two lines represents the change in phase velocity for a unit change in velocity of the fluid. For the Scholte wave, the sensitivity was determined to be 1.02 , which agrees well with the predicted value from the analytical model of 0.94 . The discrepancy arises from errors made while forming the water-glycerol mixtures. 
The same experiment was performed at a different frequency of $12.7 \mathrm{MHz}$ to generate the first guided mode. The change in phase of the guided mode and the theoretical change in phase for a bulk pressure wave are plotted in Figure 96.

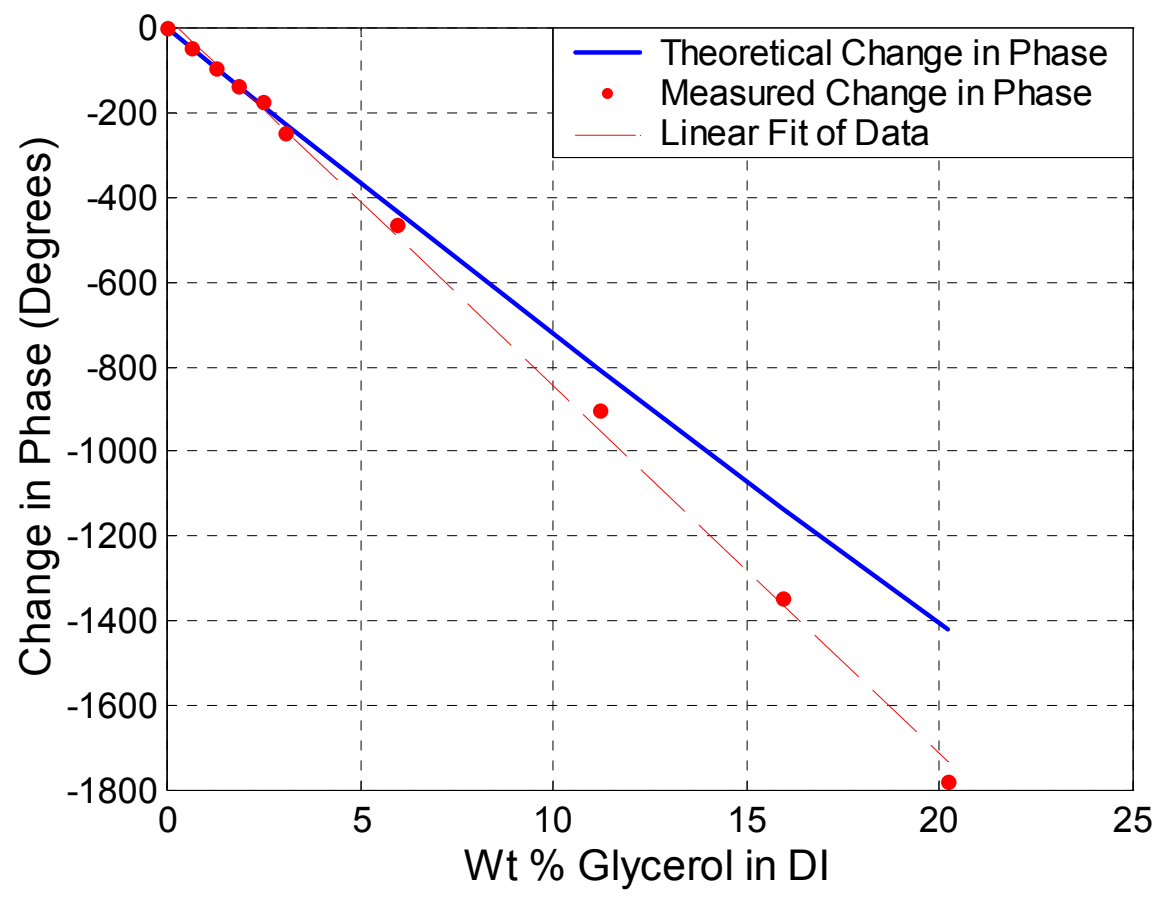

Figure 96 Change in phase of the received guided wave resulting from a change in the concentration of glycerol in de-ionized water.

From the plot, it is obvious that the guided channel mode has a different sensitivity to changes in fluid sound speed as compared to the Scholte wave. The sensitivity was determined to be 1.23 , as compared to the analytical model prediction of 1.74 . The discrepancy can be attributed to differences in geometry and material properties. Since the guided mode has a different sensitivity to changes in fluid sound speed, it provides an independent equation which can be used to determine the properties of the fluid. 


\section{Sensitivity to Changes in Temperature}

It is well known that the speed of sound in water is a strong function of temperature [68]. Assuming that there are no changes in sound speed due to changes in the composition of the fluid, interdigital cMUTs can be used to measure the temperature of fluid in a microchannel or a fluid half-space. The speed of sound as a function of temperature is plotted in Figure 97 [69].

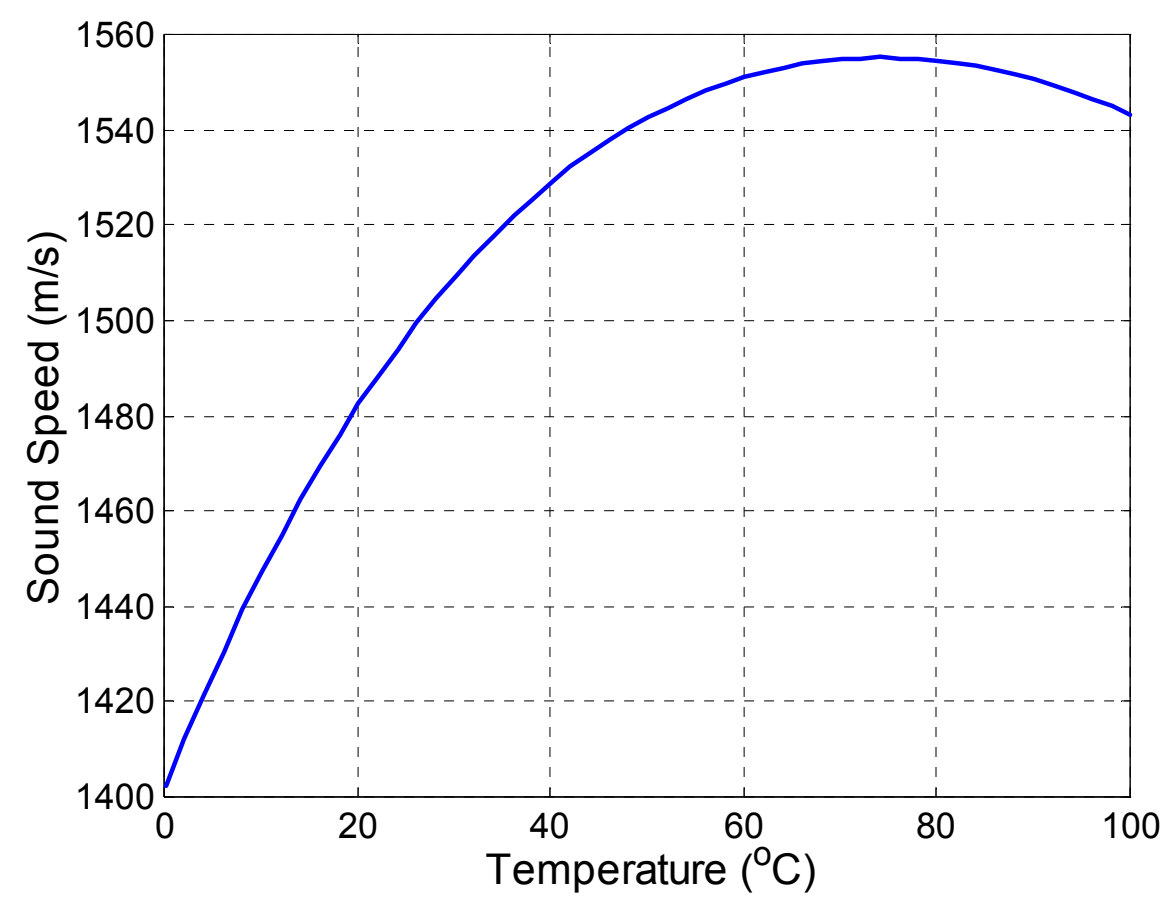

Figure 97 Sound speed in pure water as a function of temperature.

From the plot, one can see that the sound speed reaches a maximum at approximately $76^{\circ} \mathrm{C}$ and then begins to decrease. This presents a problem if one is attempting to measure temperature over a large temperature range since the function for sound speed is multi- 
valued. However, for the temperature range of $1-61^{\circ} \mathrm{C}$ the function for sound speed is single-valued and can thus be easily measured.

For small changes in fluid temperature around a median value of $26^{\circ} \mathrm{C}$, the sound speed is essentially a linear function of temperature which can be approximated as

$$
c_{\text {Water }}=2.625 T+1431
$$

where $T$ is the temperature in degrees Celsius. From Equation 83, one can see that the sensitivity of the speed of sound to changes in temperature is $2.625 \mathrm{~m} / \mathrm{s} /{ }_{0} \mathrm{C}$. Since the zero crossing measurement system has a velocity measurement accuracy of $0.25 \mathrm{~m} / \mathrm{s}$, the system should be capable of measuring temperature changes with a sensitivity of $10.5^{\mathrm{ns}} /{ }_{\mathrm{o}} \mathrm{C}$.

To verify the sensitivity to changes in temperature, a zero-crossing measurement was performed with an interdigital cMUT placed in a heated beaker of water, as shown in Figure 98. Additionally, the experiments demonstrate the unique nature of the Scholte wave and the ability to sense changes in the properties of a semi-infinite fluid. 


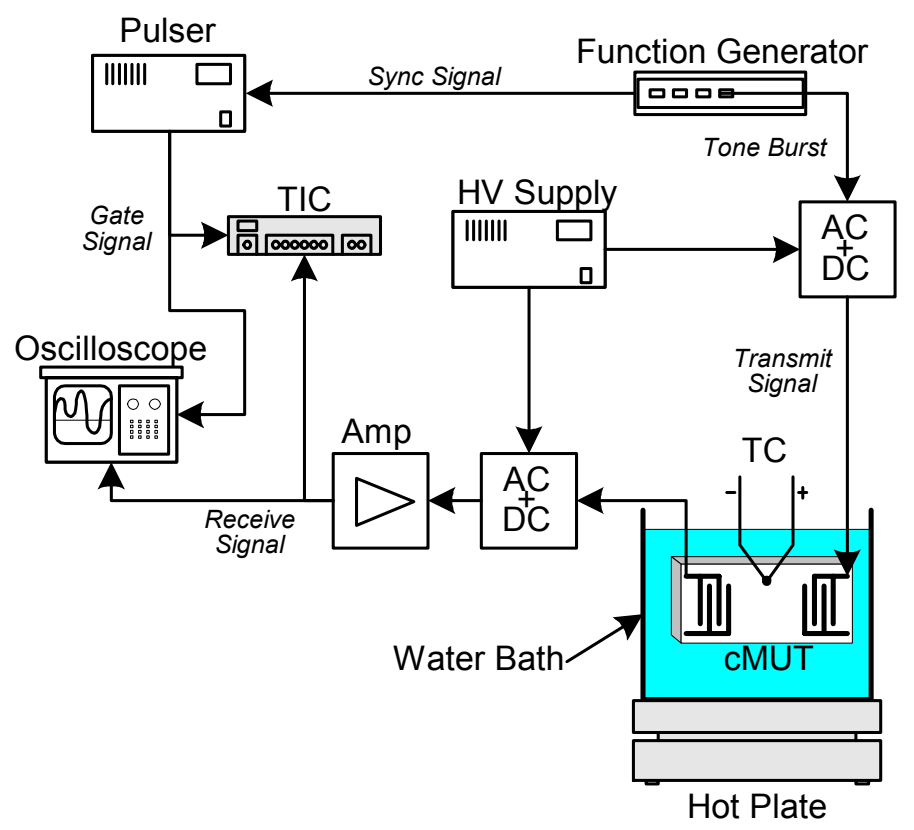

Figure 98 Illustration of setup used for temperature measurement using the Scholte wave.

The water was heated using a hotplate, and the temperature was controlled and measured using a calibrated thermocouple. The cMUT was immersed in the water at room temperature $\left(23.6^{\circ} \mathrm{C}\right)$ to establish the baseline for the measurement. Then, the temperature was gradually increased to $27^{\circ} \mathrm{C}$. While the temperature was ramping up to the desired value, the water was stirred to ensure that there were no temperature gradients in the fluid. Once an equilibrium temperature was reached, the stirrer was turned off and the measurement was made. The phase velocity of the Scholte wave as a function of temperature is plotted in Figure 99. 


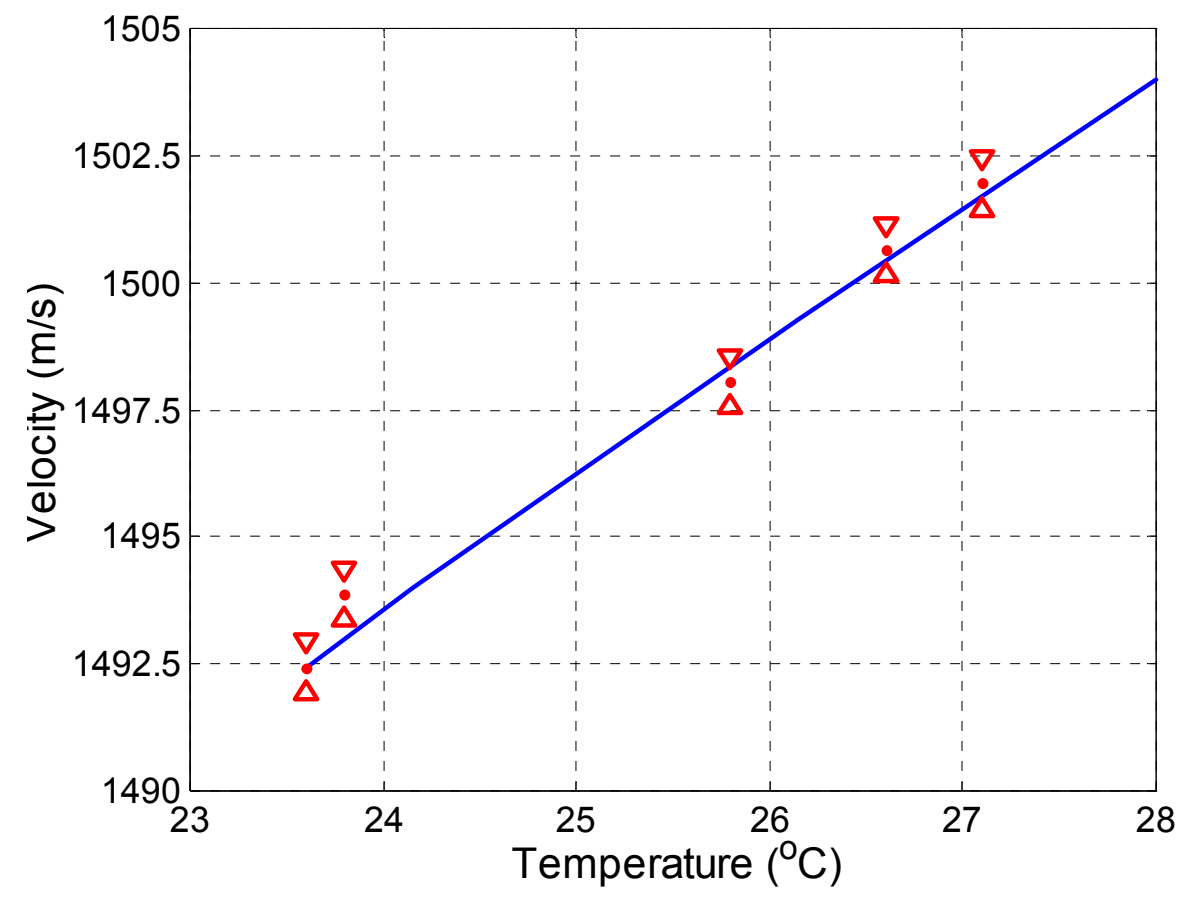

Figure 99 Scholte wave phase velocity as a function of temperature. The solid line represents the published values for the speed of sound in water as function of temperature. The error bars result from the jitter in the measurement.

From the plot, one can see that the phase velocity of the Scholte wave follows the same trend as the speed of sound in the fluid. This is expected since the sensitivity of the Scholte wave velocity to changes in fluid sound speed is 0.997 for a fluid half-space. A linear curve fit of the experimental data results in the following equation,

$$
c_{\text {meas }}=2.575 T+1432 \text {. }
$$

This matches well with the equation derived from sound speed values from the literature (Equation 83). The discrepancy results from small temperature gradients in the fluid since the thermocouple could not be placed directly in the path of the Scholte wave.

This measurement is particularly interesting since the sound speed of the fluid can be monitored using two transducers on a single substrate. Typical sound speed 
measurements are performed using two separate transducers separated by a prescribed distance and immersed in the fluid of interest [70]. The accuracy of the measurements is primarily determined by the measurement accuracy of the distance between the transmitter and receiver. The use of the Scholte wave allows one to make similar measurements using two transducers on a single substrate separated by a precise, lithographically defined distance.

\section{Sensitivity to Changes in Flowrate}

To demonstrate that flow rates in the range of milliliters per minute could be measured, a syringe pump was used to push fluid water through the microchannel with flow rates ranging from 0 to $4 \mathrm{~mL} / \mathrm{min}$. The maximum flow rate of $4 \mathrm{~mL} / \mathrm{min}$ was established by the strength of the fluid connections on the test device. The change in flow was measured by monitoring the change in effective sound speed, which can be written as

$$
c_{\text {effective }}=U_{\text {mean }}+c_{0}
$$

where $c_{\text {effective }}$ is the effective sound speed, $U_{\text {mean }}$ is the mean flow speed, and $c_{0}$ is the sound speed of the fluid [71]. Here the positive direction for the mean flow velocity points from the transmitter toward the receiver.

For flow in a $100 \mu \mathrm{m}$ deep and $750 \mu \mathrm{m}$ wide microchannel, the mean flow velocity for a flow rate of $1 \mathrm{~mL} / \mathrm{min}$ can be calculated to be approximately $0.22 \mathrm{~m} / \mathrm{s}$. This is near the accuracy limit of the system, which is $0.25 \mathrm{~m} / \mathrm{s}$. The waveforms resulting from the zero-crossing experiment for the Scholte wave with various flow rates are presented in Figure 100 . 


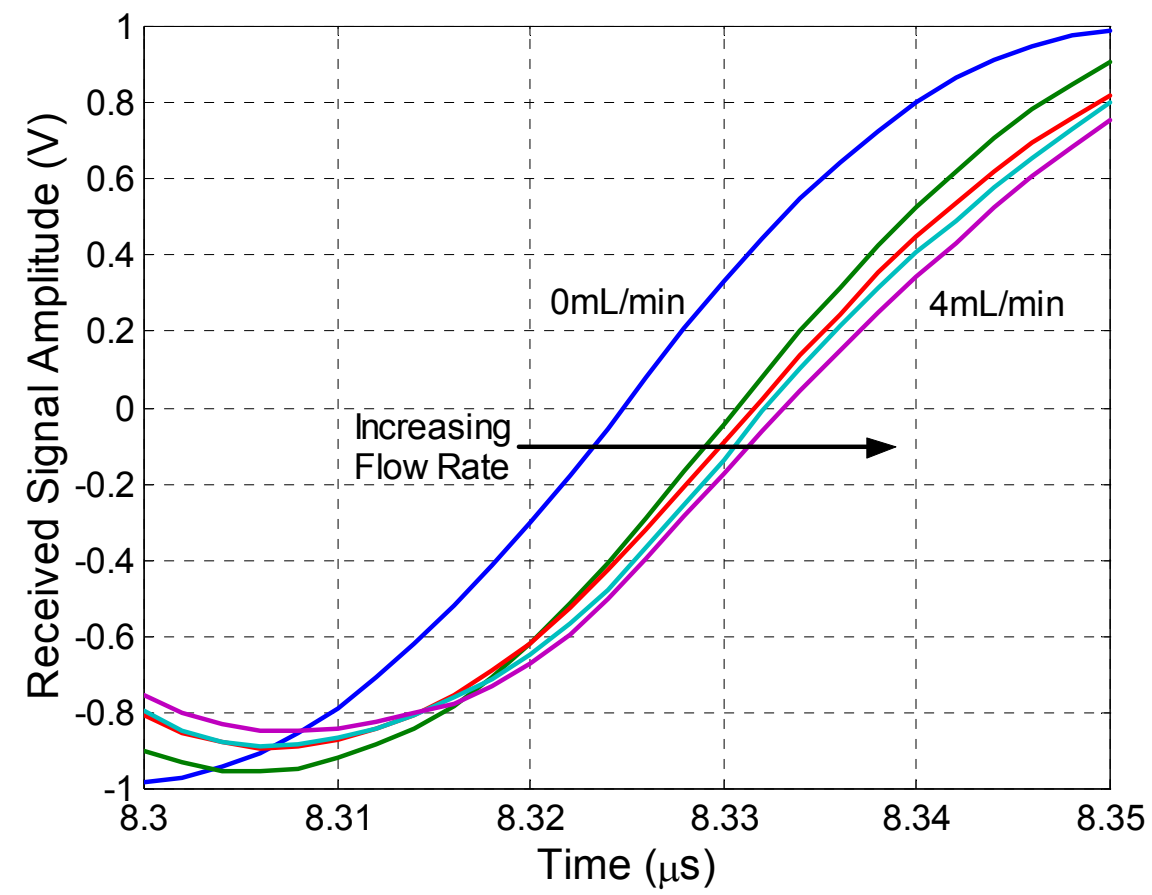

Figure 100 Received waveforms for Scholte wave transmit-receive measurements in pure water with flow rates ranging from 0 to $4 \mathrm{~mL} / \mathrm{min}$ with $1 \mathrm{~mL} / \mathrm{min}$ increments.

For the results shown in Figure 100, the flow was directed from the receiver toward the receiver, decreasing the effective sound speed of the fluid. In the results there is a $6 \mathrm{~ns}$ jump from the zero flow curve to the $1 \mathrm{~mL} / \mathrm{min}$ curve. This results from a linearity problem in the syringe pump. However, for increasing flow rates after $1 \mathrm{~mL} / \mathrm{min}$, the zero crossing shifts in time in approximately $1 \mathrm{~ns}$ increments as expected. The minimum detectable flow rate can be decreased by improving the SNR of the system, thus further decreasing the jitter. 


\section{$\underline{\text { Multi-Mode Ultrasonic Fluid Sensor }}$}

It has been shown that the same interdigital cMUT can be used to perform Scholte wave and guided mode transmit receive measurements in fluid-filled microchannels. When coupled with standard pulse-echo measurements, one obtains three independent measurements that can be used to extract the properties of the fluid such as sound speed, viscosity and flow rate. The different types of measurements are illustrated in Figure 101.

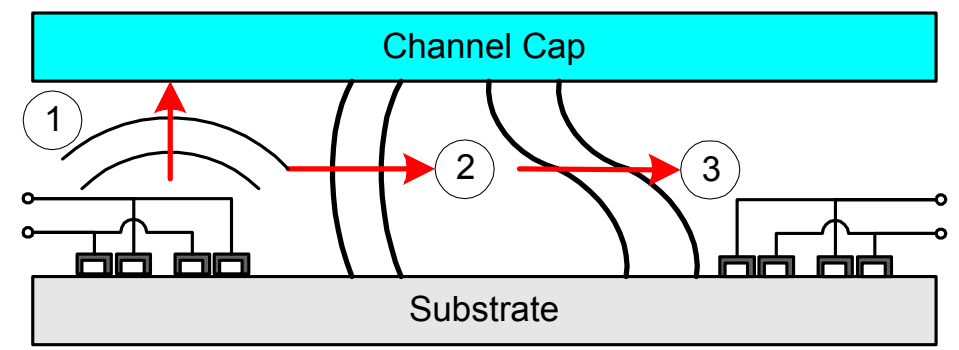

Figure 101 Illustration of independent modes that can be excited in a microfluidic channel: 1) Bulk wave pulse-echo, 2) Scholte wave transmit-receive, 3) Guided mode transmit-receive.

The pulse-echo measurement is performed by connecting the legs of a single transducer and exciting them with a short voltage pulse. The transmit-receive measurements are performed by exciting the legs of the transducer with phase shifted tone bursts at the frequency required to generate the guided mode of interest.

Since each measurement has different sensitivities to changes in geometry and fluid properties, they must be carefully selected to obtain meaningful measurements. The parameters that strongly affect the various measurements are listed in Table 9. 
Table 9 Measurable quantities and dependent variables for various acoustic measurements

\begin{tabular}{||c|c|c|}
\hline Measurement & Measurable Quantities & Dependent Variables \\
\hline Pulse-echo & Arrival time & $\mathrm{c}_{0}, \mathrm{~h}$ \\
\hline Scholte transmit-receive & Phase velocity, Amplitude & $\mathrm{c}_{0}, \mathrm{U}_{\text {mean }}, \mathrm{A}$ \\
\hline Guided transmit-receive & Phase velocity, Amplitude & $\mathrm{c}_{0}, \mathrm{U}_{\text {mean }}, \mathrm{A}, \mathrm{h}$ \\
\hline
\end{tabular}

The pulse-echo measurement monitors the arrival time of a reflected acoustic pulse and is thus sensitive to changes in fluid sound speed, $c_{0}$, and the reflector height, $h$. The pulseecho measurement is insensitive to changes in the mean velocity of the flow, $U_{\text {mean }}$. With the Scholte wave transmit-receive measurement, one can monitor the amplitude and phase velocity of the received signal. The phase velocity is dependent upon $c_{0}$ and $U_{\text {mean }}$, and the amplitude is dependent upon the attenuation coefficient, $A$. The phase velocity of guided wave, is also dependent on $c_{0}$ and $U_{\text {mean }}$, but is also strongly dependent on the channel height as illustrated by the dispersion curve in Figure 25. The amplitude of the received signal is also dependent upon the attenuation coefficient, $A$.

Since the Scholte wave is only slightly dispersive with respect to channel height (Figure 25), it can be used to determine the flow rate, independent of the fluid properties. The flow rate can be determined by performing two transmit-receive measurements: one sends a Scholte wave tone burst in the direction of the flow and the other sends the tone burst against the flow. The wave traveling with the flow has a higher effective sound speed,

$$
c_{\text {With Flow }}=c_{0}+U_{\text {mean }}
$$

while the wave traveling against the flow has a lower effective sound speed,

$$
c_{\text {Against Flow }}=c_{0}-U_{\text {mean }} \text {. }
$$

The resulting waveforms are shifted in time as shown in Figure 102. 


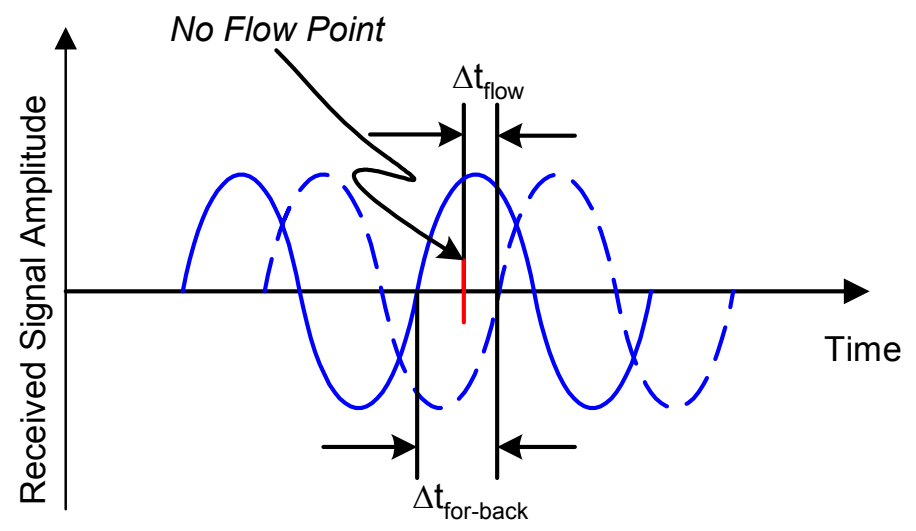

Figure 102 Illustration of independent flow measurement.

Since the mean flow changes the effective sound speed linearly, one can monitor the relative change in mean flow velocity by dividing the total measured time shift by two,

$$
\Delta t_{\text {flow }}=\frac{1}{2} \Delta t_{\text {for-back }} .
$$

This measurement can be performed using the same zero-crossing technique previously described. Once the mean flow velocity is know, one can measure the change in phase velocity of the Scholte wave to determine the fluid sound speed. Then the phase velocity of the guided wave can be measured to determine the channel height, since the contributions of flow and fluid sound speed can be factored out of the measured data.

The amplitude of an acoustic signal decreases as it propagates due to attenuation. The pressure amplitude as a function of distance can be written as

$$
p=p_{0} e^{-\alpha x}
$$

where $p_{0}$ is pressure amplitude at the reference position and $\alpha$ is the frequency dependent attenuation coefficient. In general, $\alpha$ has contributions from viscous losses, heat conduction, and relaxation $[72,73,74]$. For liquids, the attenuation due to viscous effects 
is several orders of magnitude larger than the other effects for the frequency range of interest. Neglecting these effects, the attenuation coefficient can be written as

$$
\alpha=\left(\frac{4}{3}+\frac{\mu_{B}}{\mu}\right) \frac{\mu \omega^{2}}{2 c_{0}^{3} \rho_{0}}
$$

where $\mu$ is the dynamic viscosity, $\mu_{B}$ is the bulk viscosity, and $\omega$ is the angular frequency.

Removing the dependence of $\alpha$ on $f^{2}$ yields the more familiar attenuation coefficient $A$ :

$$
A=\left(\frac{4}{3}+\frac{\mu_{B}}{\mu}\right) \frac{2 \mu \pi^{2}}{c_{0}^{3} \rho_{0}} .
$$

If the amplitude of the received signal is measured and compared with a calibration standard, one can determine the attenuation coefficient for the fluid. This measurement can be repeated at different frequencies corresponding to the different acoustic modes to increase the accuracy of the measured attenuation coefficient.

The most important aspect regarding the implementation of this multi-mode acoustic sensor is calibration. To be able to accurately measure the fluid sound speed, flow velocity, and attenuation, one must perform a thorough calibration of the system.

\section{$\underline{\text { Discussion }}$}

The ability of interdigital capacitive micromachined ultrasonic transducers to excite guided acoustic waves in fluid half-spaces and microchannels has been demonstrated. A single transducer is used to selectively excite different guided acoustic waves by changing the frequency so that the transducer periodicity matches the wavelength of the mode to be generated. The various acoustic modes are then used to sense changes in fluid sound speed, flow rate, and temperature by monitoring the phase 
velocity of the propagating modes. The measurement system was capable of measuring changes in fluid sound speed of $0.25 \mathrm{~m} / \mathrm{s}$. When used as a temperature sensor, these transducers can measure temperature changes of $0.1^{\circ} \mathrm{C}$. These values compare favorably with the minimum detectable change in sound speed and temperature reported by Jagannathan et al. [15]. These researchers used a pulse-echo technique in fluid-filled microchannels to measure changes in fluid sound speed and temperature. The minimum measurable change in sound speed that was reported was $1.2 \mathrm{~m} / \mathrm{s}$ which corresponds to a change in water temperature of $1.7^{\circ} \mathrm{C}$.

The minimum detectable change in flow rate that could be resolved by the guided mode flow sensor was $1 \mathrm{~mL} / \mathrm{min}$. Joshi et al. report the use of a SAW oscillator as a thermal flow sensor [75]. In their research, the substrate is heated and the decrease in plate temperature resulting from the fluid flow is measured by monitoring the frequency shift of the oscillator. This system was capable of measuring changes in flow rate of $0.1 \mu \mathrm{L} / \mathrm{min}$, which is significantly more sensitive than the interdigital cMUT flow sensor. However, Eren reports that the sensitivity of large-scale transmit-time ultrasonic flow meters is approximately $0.13 \mathrm{~m} / \mathrm{s}$ [76]. Although some flow and fluid sensing techniques are more sensitive than the guided mode sensors developed in this research, these transducers have a distinct advantage: the ability of a single sensor to determine a variety of flow and fluid properties. The same interdigital cMUT can measure fluid sound speed, flow rate, and channel height.

In summary, interdigital cMUTs can be used in microfluidic environments to determine the flow and fluid properties by performing pulse-echo measurements in addition to Scholte wave and guided mode transmit-receive measurements. The 
transducers are also attractive for measuring the properties of semi-infinite fluids using the Scholte wave. Since both the transmitter and receiver are located on the same substrate, the transducer can be immersed in the fluid of interest like a "dipstick" and used to measure the fluid's visco-elastic properties. 


\section{CHAPTER 8}

\section{FLUID PUMPING}

Since the Scholte wave propagates in microchannels for all frequency-channel height combinations, as shown in the dispersion curve of Figure 25, it is a suitable mode for acoustic actuation applications such as pumping. The high directionality resulting from the phased excitation scheme allows the majority of the acoustic power in the liquid to be directed into a preferred lateral direction. When the amplitude of the acoustic wave is large, acoustic streaming forces result in a net flow of the fluid in the direction of the traveling wave.

In the following section, the phenomenon of acoustic streaming is described in detail. Then, the performance of interdigital cMUTs used as acoustic streaming pumps is presented and discussed. 


\section{$\underline{\text { Acoustic Streaming }}$}

Acoustic streaming is nonlinear acoustic effect that occurs when a high amplitude acoustic wave propagates through a dissipative fluid medium. As the acoustic wave travels through the fluid, the amplitude of the wave decreases due to attenuating effects such as viscous losses, molecular relaxation, and interaction with rigid boundaries. This attenuation results in a gradient in the momentum flux which in turn generates a Reynolds stress in the fluid. The Reynolds stress results in a steady motion of the fluid [77], which cannot occur if there is no attenuation in the fluid.

\section{Governing Equations}

The acoustic streaming equations are developed following the approach of Nyborg [78]. In the development, it is assumed that the fluid is homogeneous and isotropic. The dynamic equation for a small volume element of fluid is found by equating the surface forces acting on the fluid due to elasticity and viscosity to the acceleration of the fluid particle:

$$
\vec{f}=\rho\left[\frac{\partial \vec{u}}{\partial t}+(\vec{u} \cdot \vec{\nabla}) \vec{u}\right]
$$

where $\vec{f}$ is the net force per unit volume due to stresses, $\rho$ is the density, and $\vec{u}$ is the velocity. For a Newtonian viscous fluid, the net force per unit volume is written using the Navier-Stokes equation as

$$
\vec{f}=-\vec{\nabla} p+\left[\mu_{B}+\frac{4}{3} \mu\right] \vec{\nabla} \vec{\nabla} \cdot \vec{u}-\mu \vec{\nabla} \times \vec{\nabla} \times \vec{u}
$$


where $\mu$ and $\mu_{B}$ are the shear and bulk viscosity coefficients for the fluid and the body force term has been omitted. The continuity equation,

$$
\frac{\partial \rho}{\partial t}+\nabla \cdot(\rho \vec{u})=0
$$

is then combined with Equations 92 and 93 to produce the following

$$
\frac{\partial(\rho \vec{u})}{\partial t}-\vec{F}^{\prime}=-\vec{\nabla} p+\left[\mu_{B}+\frac{4}{3} \mu\right] \vec{\nabla} \vec{\nabla} \cdot \vec{u}-\mu \vec{\nabla} \times \vec{\nabla} \times \vec{u}
$$

where

$$
-\vec{F}^{\prime}=\rho(\vec{u} \cdot \vec{\nabla}) \vec{u}+\vec{u} \vec{\nabla} \cdot \rho \vec{u}
$$

The right hand side of Equation 95 represents the net force applied to the fluid element resulting from stresses acting on the surface of the element. Lighthill noted that the $\vec{F}^{\prime}$ term is the divergence of the momentum flux tensor, which is ultimately responsible for acoustic streaming [77].

To obtain the time-invariant solution for the streaming field, one must take the time average of Equation 95 which results in

$$
\vec{F}=\vec{\nabla}\langle p\rangle-\mu \nabla^{2}\langle\vec{u}\rangle
$$

where the left hand side is given by

$$
\vec{F}=-\langle\rho(\vec{u} \cdot \vec{\nabla}) \vec{u}+\vec{u} \vec{\nabla} \cdot \rho \vec{u}\rangle .
$$

The \langle\rangle notation represents the time average and $\left\langle-\vec{F}^{\prime}\right\rangle$ has been replaced with $\vec{F}$ for convenience. The term $\vec{F}$ represents the time-averaged rate of increase of momentum flux for the fluid element. 
The method of successive approximations can be employed to solve Equation 98. It is assumed that the excess pressure, density, and velocity at any point in the fluid can be written as a series such as:

$$
\begin{gathered}
P-P_{0}=p_{1}+p_{2}+\ldots \\
\rho-\rho_{0}=\rho_{1}+\rho_{2}+\ldots \\
\vec{u}=\vec{u}_{1}+\vec{u}_{2}+\ldots
\end{gathered}
$$

In these equations, the terms $p_{1}, \rho_{l}$, and $\vec{u}_{1}$ are the first order approximations to the field variables and represent the linear sound field. The series representation of the field variables is substituted into Equation 98 and terms of like order are collected. Collecting the zero order terms, one finds that the $P_{0}$ is constant. No equation is obtained from the first order terms, but collecting the second order terms yields a valuable result: the equation for the streaming velocity, $\vec{u}_{2}$, in terms of the first order field variables. This equation can be written as

$$
\vec{F}_{2}=\vec{\nabla} p_{2}-\mu \nabla^{2} \vec{u}_{2}
$$

where

$$
\vec{F}_{2}=-\rho_{0}\left\langle\left(\vec{u}_{1} \cdot \vec{\nabla}\right) \vec{u}_{1}+\vec{u}_{1}\left(\vec{\nabla} \cdot \vec{u}_{1}\right)\right\rangle
$$

$\vec{F}_{2}$ is referred to as the effective force density and can be calculated when $\vec{u}_{1}$ is known.

\section{$\underline{\text { Acoustic Streaming Field in a Rectangular Microchannel }}$}

The acoustic streaming field generated by a plane wave propagating in the fluid-

filled region between two parallel, rigid walls can be used to approximate the field in a fluid filled microchannel. The Scholte wave can be approximated as a plane wave since 
its amplitude is essentially constant over the thickness of the channel, as shown in the mode shape in Figure 26. The geometry of the model is illustrated in Figure 103.

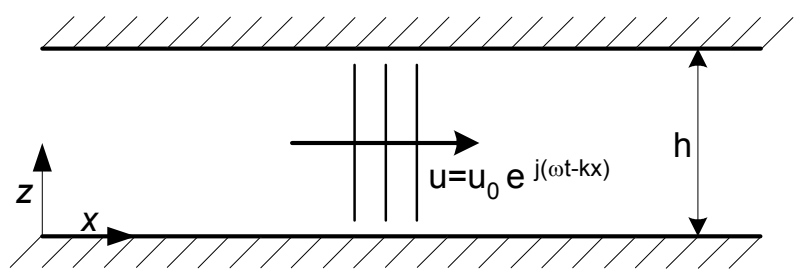

Figure 103 Illustration of the analytical model for acoustic streaming.

It is assumed that the traveling wave is time harmonic and that the channel width is much greater than its height. A no-slip boundary condition is enforced at the fluid-solid interfaces so that all components of the velocity are zero there. The first order velocity field is obtained by approximating Equation 95 to first order and combining the result with the first order approximation to the state equation. The solution for the first order velocity field is then substituted into Equation 103 to obtain the force density distribution. If the channel has open ends, the gradient of the second order pressure is zero. Since there can be no net flow in the z-direction, the second order velocity can only have an $\mathrm{x}$ component. Therefore, Equation 103 can be rewritten as

$$
F_{2 x}=-\mu \nabla^{2} u_{2 x}
$$

The force density in the x-direction, $F_{2 x}$, has two components which result from viscous attenuation in the channel, $F_{2 x a}$, and the acoustic boundary layer at the fluid-solid interfaces, $F_{2 x b}$. The total force density is the sum of these two components,

$$
F_{2 x}=F_{2 x a}+F_{2 x b}
$$


The component, $F_{2 x a}$, can be written as

$$
F_{2 x a}=\alpha \rho_{0} u_{a 0}^{2} e^{-2 \alpha x}
$$

where $\alpha$ is the viscous attenuation coefficient for the fluid, which can be written as

$$
\alpha=\left(\frac{4}{3}+\frac{\mu_{B}}{\mu}\right) \frac{\mu \omega^{2}}{2 \rho_{0} c_{0}^{2}} .
$$

In Equation 107, $\mu_{B}$ is the bulk viscosity, $\mu$ is the dynamic viscosity, $\omega$ is the angular frequency, $\rho_{0}$ is the density and $c_{0}$ is the fluid sound speed. The force density component $F_{2 x b}$ can be written as

$$
F_{2 x b}=\frac{1}{2} F_{2 x a}\left[\frac{k}{\alpha} f_{1}(n)+f_{2}(n)\right]
$$

where

$$
\begin{gathered}
f_{1}(n)=C+S-e^{-2 n} \\
f_{2}(n)=e^{-2 n}-3 C+S .
\end{gathered}
$$

The quantities $C, S$, and $n$ are

$$
\begin{gathered}
C=e^{-n} \cos (n) \\
S=e^{-n} \sin (n) \\
n=\kappa z .
\end{gathered}
$$

Here $\kappa$ is defined as

$$
\kappa=\sqrt{\frac{\omega \rho_{0}}{2 \mu}}
$$

and $1 / \kappa$ is the acoustic boundary layer thickness. 
To obtain the solution for the streaming velocity resulting from the acoustic boundary layer, the viscous force density distribution, $F_{2 x a}$, is set equal to zero and Equation 104 is solved using the first order velocity field to yield

$$
u_{2 b}=\left(\frac{u_{a 0}^{2}}{4 c_{0}}\right) f_{3}(n), \quad f_{3}(n)=1+2(S-C)+e^{-2 n} .
$$

The streaming field resulting from the acoustic boundary layer decays very quickly as a function of distance from the wall. After several multiples of the acoustic boundary layer thickness, the streaming field reaches a constant value of $u_{a 0}{ }^{2} / 4 c_{0}$.

The streaming velocity resulting from the viscous attenuation of the plane wave in the channel is found by setting the boundary layer contribution of the force density equal to zero and solving Equation 104. The streaming velocity is found to be

$$
u_{2 a}(z)=C_{1} n_{1}\left(1-n_{1}\right), \quad n_{1}=\frac{z}{h}
$$

where

$$
C_{1}=\frac{\alpha \rho_{0} u_{a 0}^{2} h^{2}}{2 \mu} .
$$

The two quantities that can be altered to increase the streaming velocity for a given fluid are the frequency, $\omega$, and amplitude, $u_{a 0}$, of the plane wave; the streaming velocity increases with the $\omega^{2}$ and $u_{a 0}{ }^{2}$. All of the other variables are fluid properties.

The total streaming velocity in the channel is the superposition of the boundary layer and viscous streaming terms,

$$
u_{2 x}=u_{2 x a}+u_{2 x b} .
$$

The total streaming field is plotted in Figure 104 for a $200 \mathrm{kPa}$ plane wave in a $100 \mu \mathrm{m}$ water-filled channel for a range of frequencies. In Figure 104, one can see that the 
streaming velocity increases with $\omega^{2}$. Also, the streaming field is parabolic and has a maximum value of $C_{l} / 4$ at half of the channel height.

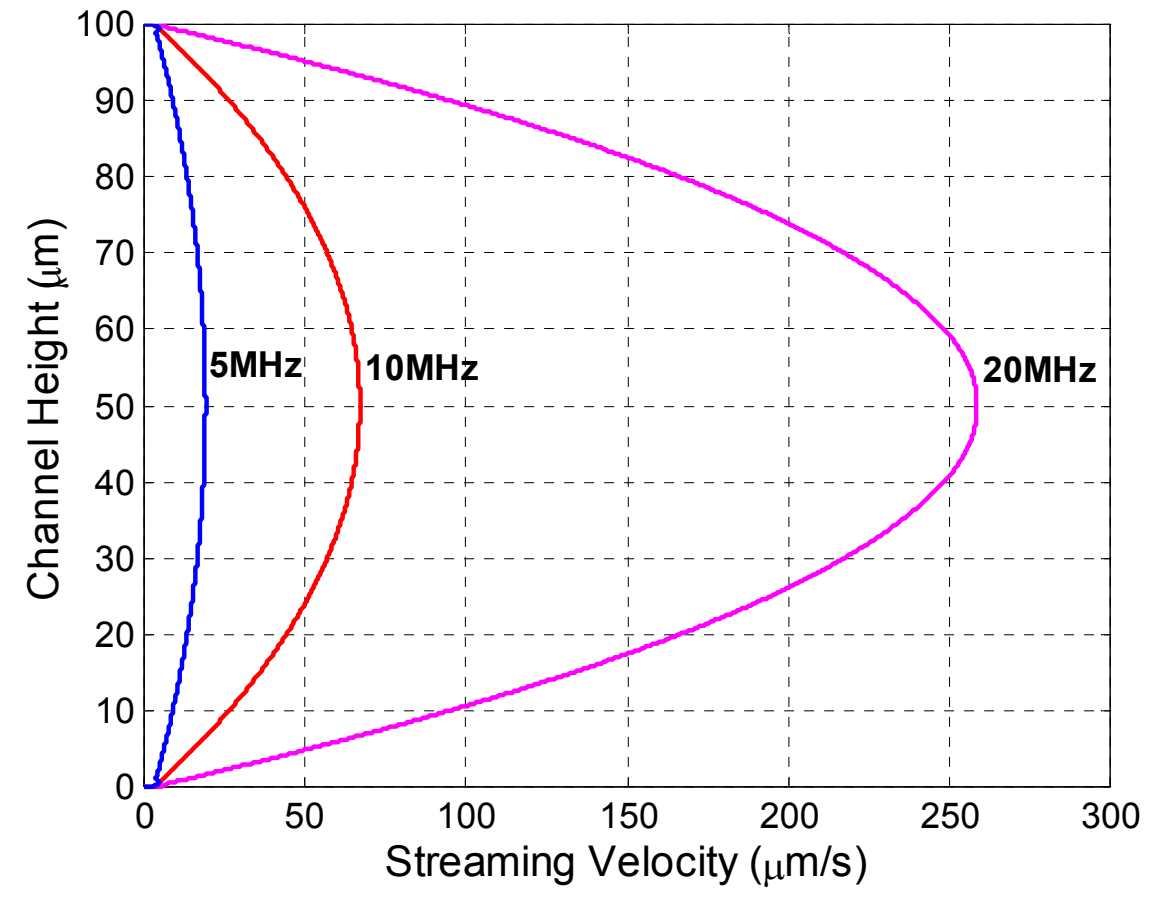

Figure 104 Plot of the theoretical acoustic streaming field in a $100 \mu \mathrm{m}$ deep, water filled channel for a range of frequencies.

The streaming field for a $10 \mathrm{MHz}$ plane wave is plotted in Figure 105 for a range of pressure amplitudes. From the plot, one can see that the streaming velocity increases with the square of the wave amplitude, thus linearly with the intensity of the plane wave. 


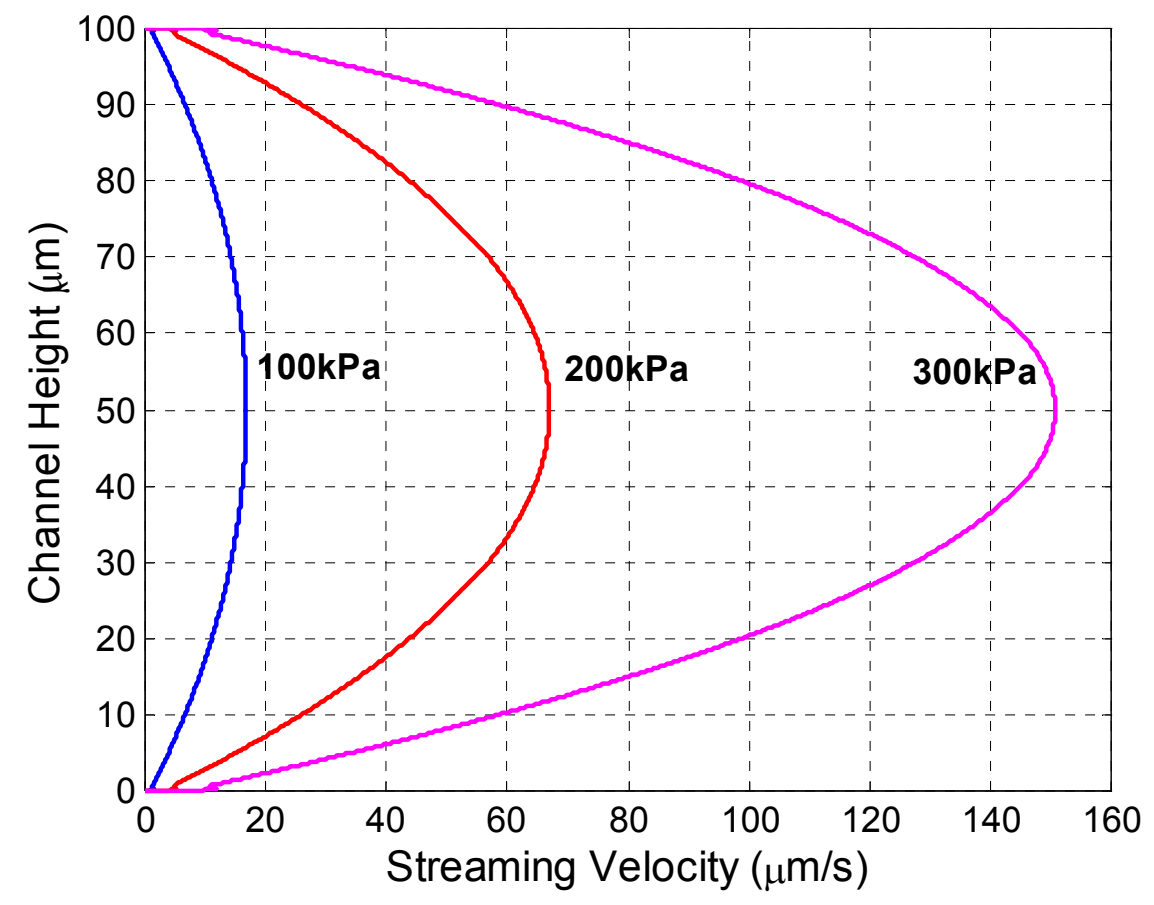

Figure 105 Plot of the acoustic streaming field in a $100 \mu \mathrm{m}$ deep, water filled channel for different plane wave amplitudes.

If one follows the argument that streaming increases with attenuation, one may infer that when a fluid with greater acoustic attenuation is subjected to the same amplitude plane wave at the same frequency, the streaming velocity will be increased. To investigate this hypothesis, the streaming fields were calculated for a $10 \mathrm{MHz}, 200 \mathrm{kPa}$ plane wave propagating in $100 \mu \mathrm{m}$ channel filled with water and glycerol and are plotted in the Figure 106. 


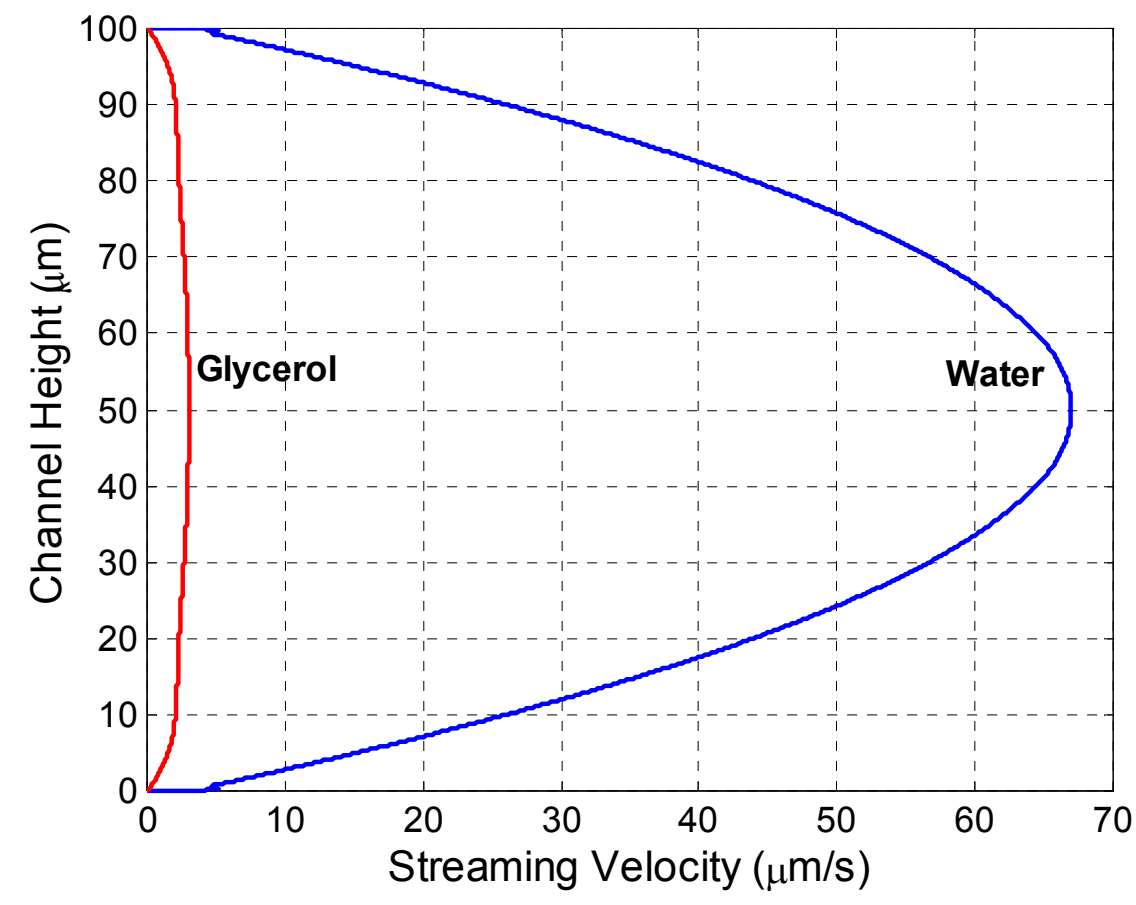

Figure 106 Plot of acoustic streaming field in a $100 \mu \mathrm{m}$ deep channel filled with pure water and pure glycerol.

Although the acoustic attenuation coefficient, $\alpha$, for glycerol is 120 times greater than that of water, the streaming velocity is reduced significantly. This is due to the fact that the increased viscosity of glycerol is primarily responsible for the increased attenuation. However, this viscosity inhibits the flow of the fluid which in turn reduces the streaming velocity. 


\section{Efficiency of Acoustic Streaming}

The analytical model of acoustic streaming can be used to predict the efficiency of an acoustic streaming-based pump. To evaluate the efficiency, one must determine the power in the streaming flow and the power in the incident plane wave. The power in the streaming flow, $P_{\text {stream }}$, is the product of the pressure induced by the streaming forces, $p_{\text {stream }}$, and the streaming induced flow rate, $Q_{\text {stream }}$,

$$
P_{\text {stream }}=p_{\text {stream }} \cdot Q_{\text {stream }}
$$

Equations 105 and 118 can be integrated over the channel height to obtain the pressure acting on the fluid and the volume flow rate, respectively. The power in the incident plane wave can be calculated from the familiar relation,

$$
P_{\text {plane }}=A \cdot \frac{p_{r m s}^{2}}{\rho_{0} c_{0}}
$$

where $A$ is the cross sectional area of the channel and $p_{r m s}$ is the root-mean-square pressure of the plane wave. The streaming efficiency, $\eta_{\text {stream }}$, is then defined as the ratio of the power in the streaming flow to the power in the plane wave,

$$
\eta_{\text {stream }}=\frac{P_{\text {stream }}}{P_{\text {plane }}} \times 100 \%
$$

The power in a $200 \mathrm{kPa}$ plane wave propagating in water-filled channel $100 \mu \mathrm{m}$ deep and $750 \mu \mathrm{m}$ wide is $0.001 \mathrm{~W}$. The power in the streaming field resulting from this wave is $1.8 \times 10^{-16} \mathrm{~W}$, yielding an efficiency of $1.7 \times 10^{-11} \%$. 
If the power in the plane wave is compared with the power in the streaming flow, one finds the following proportionality for streaming resulting from the attenuation of the free wave:

$$
\eta_{\text {Stream,Free }} \propto \frac{\alpha^{2} \rho_{0} u_{a 0}^{2}}{2 \mu c_{0}} .
$$

The acoustic streaming efficiency should improve as the amplitude of the plane wave is increased since the streaming efficiency is proportional to $u_{a 0}{ }^{2}$. Also, since the attenuation coefficient is proportional to $f^{2}$, the streaming efficiency is proportional to $f^{4}$.

A better figure of merit for acoustic streaming comes from a comparison of the amount of power in the acoustic streaming flow to the power lost by attenuation over a unit distance. The power in the attenuated plane wave at a particular x-location in the channel is

$$
P_{\text {plane }}(x)=\frac{p_{0}^{2} e^{-2 \alpha x}}{2 \rho_{0} c_{0}}
$$

and the power lost over a given distance $L$ is

$$
P_{\text {Lost }}=\frac{p_{0}^{2}}{2 \rho_{0} c_{0}}\left(1-e^{-2 \alpha L}\right) .
$$

For a $200 \mathrm{kPa}, 10 \mathrm{MHz}$ plane wave in water, the power lost over a distance of $10 \mathrm{~mm}$ is $4.9 \times 10^{-5} \mathrm{~W}$, which is $5 \%$ of the input power of the plane wave. The ratio of the power in the streaming flow to the lost power yields an efficiency of $3.6 \times 10^{-10} \%$, which is still small. The power that does not go into the streaming motion of the fluid is lost to heat [79].

For a given channel geometry and plane wave amplitude, the amount of heat generated by attenuation can be determined. The dimensions for a typical microchannel 
used for acoustic streaming experiments are $20 \mathrm{~mm}$ long, $100 \mu \mathrm{m}$ deep, and $750 \mu \mathrm{m}$ wide. The power lost due to attenuation of the same $200 \mathrm{kPa}, 10 \mathrm{MHz}$ wave over the $20 \mathrm{~mm}$ is $9.6 \times 10^{-5} \mathrm{~W}$. If the power in the streaming flow is subtracted from the overall power loss, the corresponding rate of temperature rise for the water can be calculated to be approximately $0.92^{\circ} \mathrm{C} / \mathrm{min}$, assuming that only the water is being heated.

\section{Characterization of Acoustic Streaming Pump}

The important parameters used for characterizing the performance of an acoustic streaming-based pump are the power delivered to the fluid and the streaming velocity of the fluid. With these two quantities, the efficiency of the acoustic streaming pump can be determined.

The acoustic streaming field was generated by exciting the fingers of a 10 finger, $10 \mathrm{MHz}$ transducer with two phase-shifted, continuous wave signals at the design frequency. The cMUTs were placed in a microfluidic channel $100 \mu \mathrm{m}$ deep and filled with water as shown in Figure 107. Polystyrene spheres, $5 \mu \mathrm{m}$ in diameter, were suspended in the water to allow for flow visualization. A digital camera and commercial imaging software were used to obtain a time-lapse image of the particle motion and to track the trajectory of the polystyrene particles. 


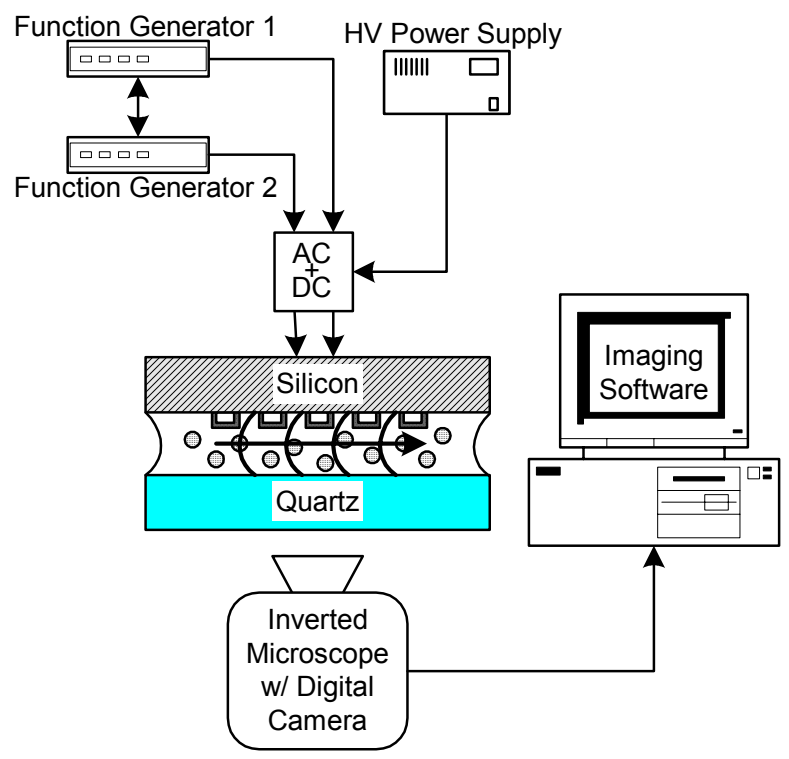

(a)

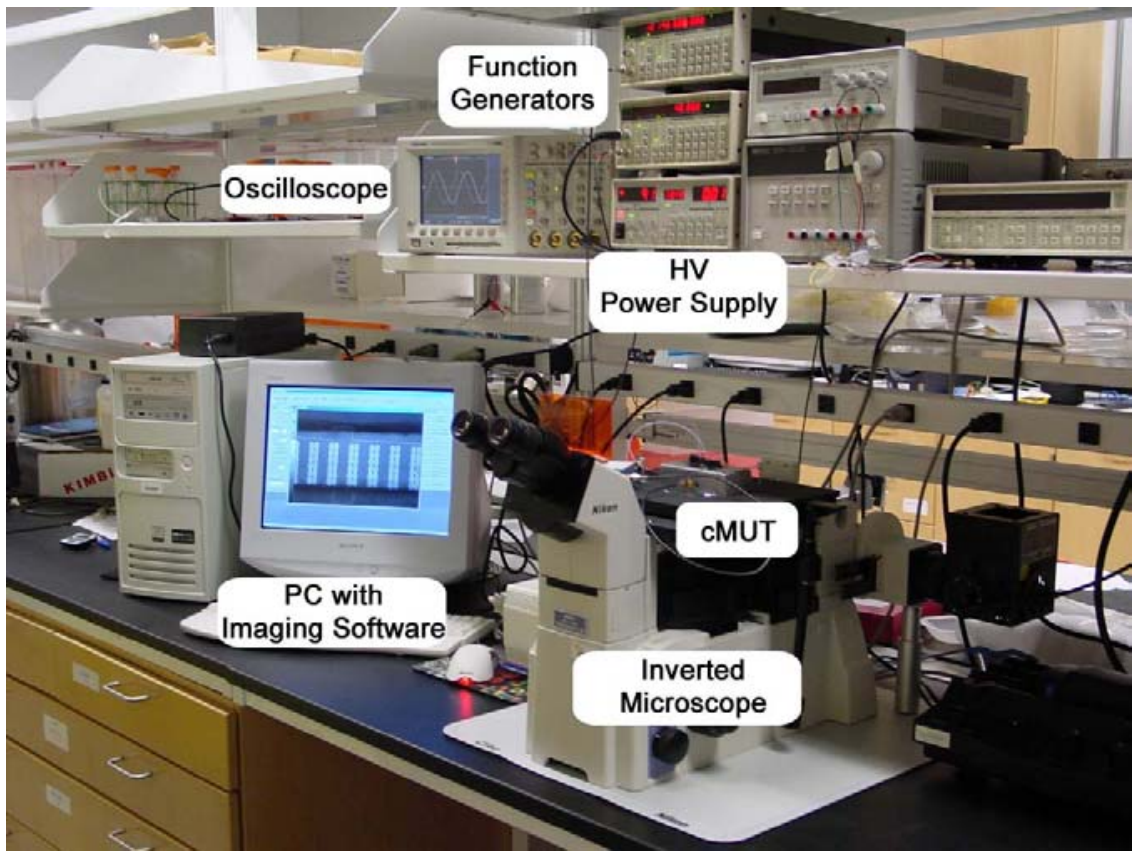

(b)

Figure 107 Schematic (a) and photograph (b) of the experimental setup used for characterizing the acoustic pump. 
The microchannel was fabricated from $100 \mu \mathrm{m}$ thick, double-stick Kapton tape [80]. The channel was formed into a 'race-track' configuration on the surface of the silicon die, as shown in Figure 108, to minimize the adverse pressure gradient.

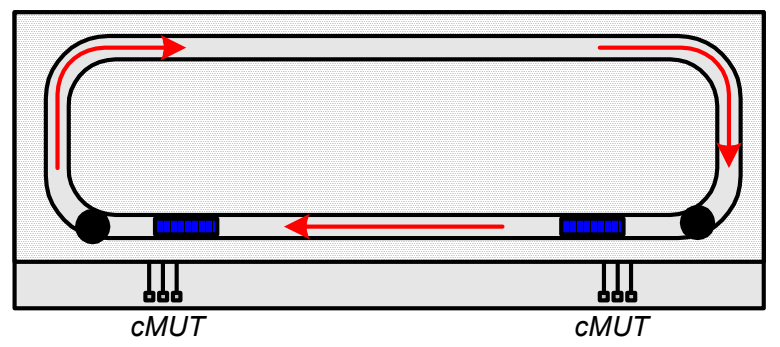

(a)

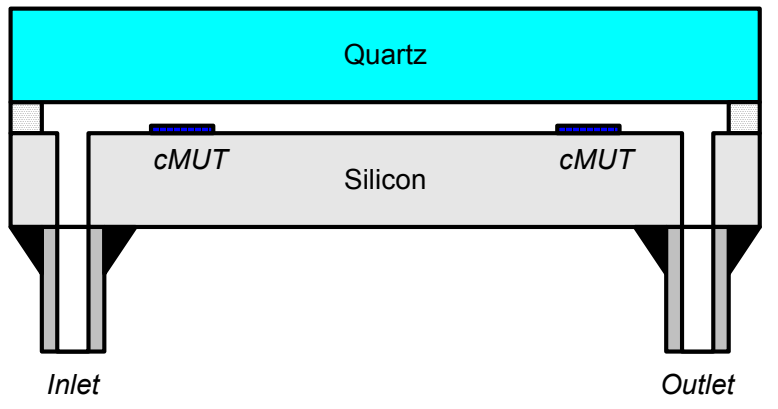

(b)

Figure 108 Microfluidic channel in race-track configuration: (a) top view showing the flow path (b) cross section showing the fluid connections.

The fluid connections were used to fill the channel and to introduce additional particles as needed. The flow velocity was measured as a function of input voltage at a location away from the immediate vicinity of the transducer to minimize the effects of acoustic radiation pressure on the particle motion.

To establish a measure of efficiency for the acoustic streaming pump, it is necessary to understand the overall flow of power in the system, which is illustrated in Figure 109. 


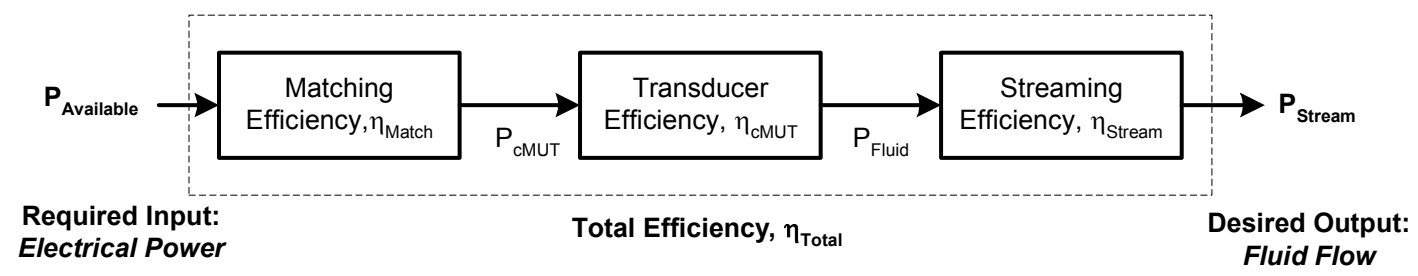

Figure 109 Illustration of power flow and various efficiencies in the acoustic streaming pump.

The power input to the system, $P_{\text {Available, }}$ is the maximum power that the AC source can deliver which can be written as

$$
P_{\text {Available }}=\frac{\left(V_{A C, R M S}\right)^{2}}{4 \cdot 50 \Omega} .
$$

The source delivers the maximum amount of power when the load impedance is equal to the source impedance which is $50 \Omega$. The matching efficiency, $\eta_{\text {Match }}$, represents how closely the load impedance, in this case the cMUT, matches the source impedance. Ideally, the matching efficiency should be unity for maximum power transfer. This efficiency can be determined experimentally by measuring the impedance of the cMUT when it is electrically tuned and then calculating the power transmission coefficient (Equation 79). The product of the available power and the matching efficiency yields the power delivered to the transducer, $P_{c M U T}$. The transducer efficiency, $\eta_{c M U T}$, is the ratio of the power delivered to the fluid to the power consumed by the transducer. The power consumed by the transducer can be determined experimentally by measuring the impedance of a cMUT with and without a bias voltage. For this measurement it is necessary to use the improved impedance measurement technique where the neighboring leg of the transducer is excited with a phase shifted signal of equal amplitude (Figure 75).When a cMUT has no DC bias, it couples very little power to the medium. Therefore, 
the power consumed when the cMUT is not biased represents the power lost in the transducer due to parasitic resistances and capacitances. The difference in power consumption with and without DC bias is the power radiated into the fluid.

The power in the streaming flow is the product of the flow rate in the channel, $Q$, and the pressure drop, $p$,

$$
P_{\text {Stream }}=Q p
$$

The flow rate is determined by measuring the maximum particle velocity using the setup shown in Figure 107, assuming a parabolic velocity profile, and integrating the velocity over the cross-sectional area of the channel. The flow resistance of the channel is dependent on the viscosity of the fluid and the geometry of the channel and can be calculated for a rectangular channel using the following relation

$$
R=\frac{12 \mu L}{w h^{3}}\left[1-\frac{h}{w}\left(\frac{192}{\pi^{5}} \sum_{n=1}^{\infty} \frac{1}{n^{5}} \tanh \left(\frac{n \pi w}{h}\right)\right)\right]^{-1}
$$

where $w$ is the channel width, $h$ is the channel height, and $L$ is the channel length [81]. The product of the flow resistance and the flow rate yields the pressure drop around the channel. The streaming efficiency is then the ratio of the power in the streaming flow to the power transferred from the cMUT to the fluid. This is a better measure than the total efficiency since it is a ratio of mechanical power in the fluid.

The flow velocity was found to increase linearly with the power consumed by the transducer, as predicted by the analytical model for acoustic streaming. The volume flow rate is plotted against the power delivered to the fluid, $P_{\text {Fluid, }}$, in Figure 110 for a 10 finger device operating $10.2 \mathrm{MHz}$. The operating frequency was changed from the design 
frequency of $10 \mathrm{MHz}$ to better match the wavelength of the Scholte wave in the $100 \mu \mathrm{m}$ channel.

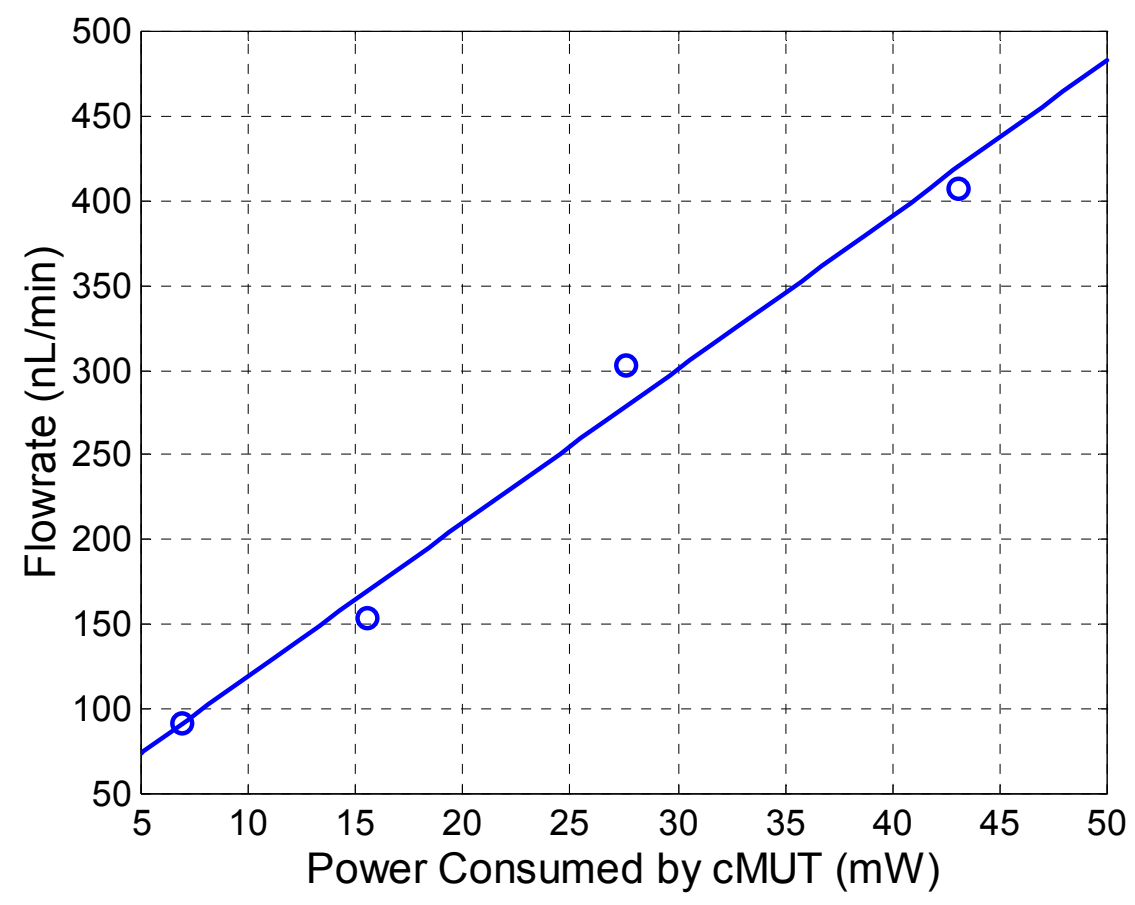

Figure 110 Volume flow rate as a function of power consumed by the cMUT.

The maximum flow rate obtained with this transducer was approximately $410 \mathrm{~nL} / \mathrm{min}$ for a power consumption of $43 \mathrm{~mW}$. The corresponding pressure head was $3.4 \mathrm{~Pa}$. The flow power was determined to be $2.3 \times 10^{-11} \mathrm{~W}$, which is of the same order as predicted by the analytical model for streaming. The streaming efficiency was found to be $5.3 \times 10^{-8} \%$. This efficiency is quite small, but one must remember that acoustic streaming is nonlinear acoustic effect. The order of magnitude of this efficiency compares well with the predictions of Equation 121. Also, the efficiency increases linearly with power as predicted by Equation 122, as shown in Figure 111 . 


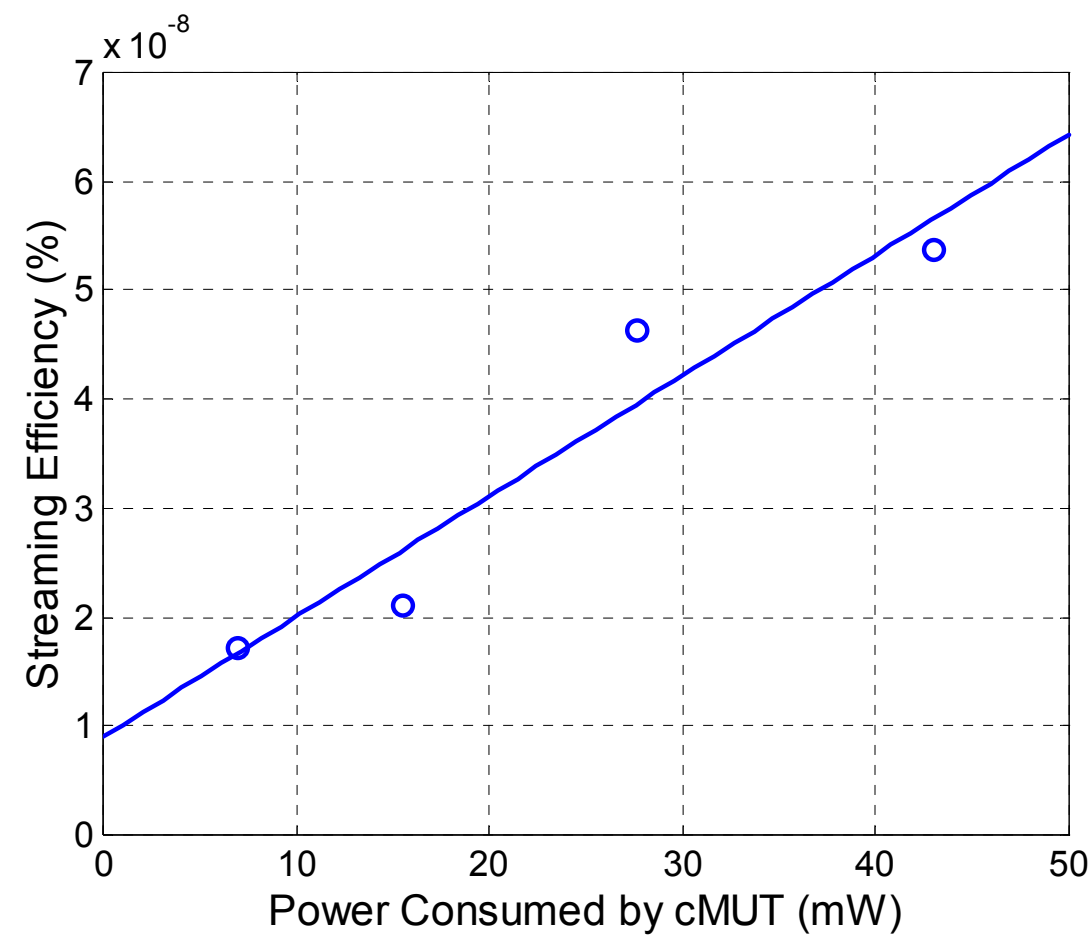

Figure 111 Streaming efficiency as a function of power consumed by the cMUT.

The various efficiencies described in Figure 109 are presented in Table 10 for a cMUT operating at $10 \mathrm{MHz}$.

Table 10 Efficiency of $10 \mathrm{MHz}$ cMUT operation at its most efficient point.

\begin{tabular}{|c|c|}
\hline Parameter & Efficiency (\%) \\
\hline Matching Efficiency & 64 \\
\hline cMUT Efficiency & 6.7 \\
\hline Streaming Efficiency & $5.3 \mathrm{e}-8$ \\
\hline Total Efficiency & $2.3 e-9$ \\
\hline
\end{tabular}

The total efficiency is the product of the matching, cMUT and streaming efficiencies. The matching efficiency can be increased to nearly $100 \%$ through proper electrical tuning. The cMUT efficiency can also be increased to nearly $100 \%$ by minimizing parasitic effects through CMOS integration. The major factor that decreases the total efficiency of the pump is acoustic streaming efficiency. The streaming efficiency is 
several orders of magnitude smaller than the other values and causes the overall efficiency to be quite low, $2.3 \times 10^{-9} \%$. The power that does not go into the streaming motion of the fluid can only be lost to heat. To verify this, the temperature of the channel was measured as a function of time using an infrared (IR) camera.

\section{$\underline{\text { Acoustic Heating }}$}

To determine how much acoustic energy is converted to heat while the interdigital cMUTs are active, it is necessary to record the temperature of the fluid in the channel as a function of time. Knowing the temperature as a function of time, the thermal properties of the liquid, and the mass of the liquid, one can estimate the rate of heat generation due to the acoustic wave propagation. For a large-scale measurement, a thermocouple would be inserted into the channel to measure the temperature in a straight-forward manner. However, this is not possible for a microfluidic channel. Therefore, an infrared (IR) camera was used to measure the temperature of the fluid in the channel as a function of time.

Since the temperature reading from the IR camera is strongly dependent on the emissivity of the material of interest, the camera must be calibrated. The system was calibrated by placing a small thermocouple in a $1 \mathrm{~mm}$ deep, water filled channel otherwise identical to the ones for the pumping experiments, as shown in Figure 112. 


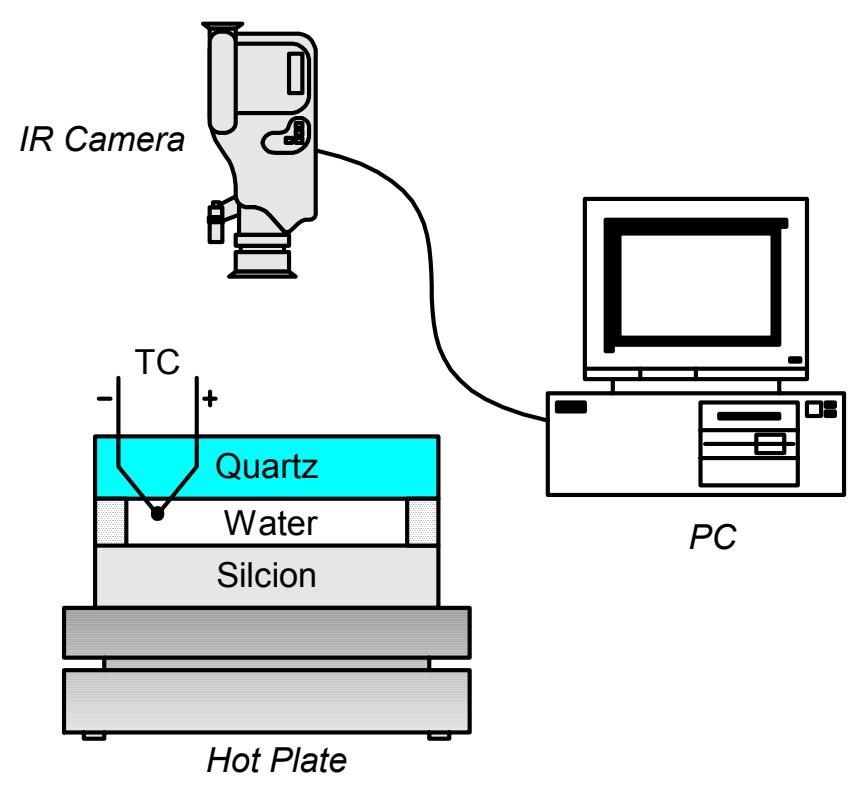

Figure 112 Calibration system used for IR camera.

The channel was placed on a hotplate, and the entire assembly was slowly heated. The camera was focused on the channel and the temperature readings from the thermocouple and IR camera were recorded for a temperature range from 23 to $43^{\circ} \mathrm{C}$ and several different values of emissivity. The thermocouple was calibrated in ice and boiling water. It was observed that changing the emissivity changed the value of the measured temperature, but did not affect the temperature difference. A typical calibration plot is shown in the following figure. 


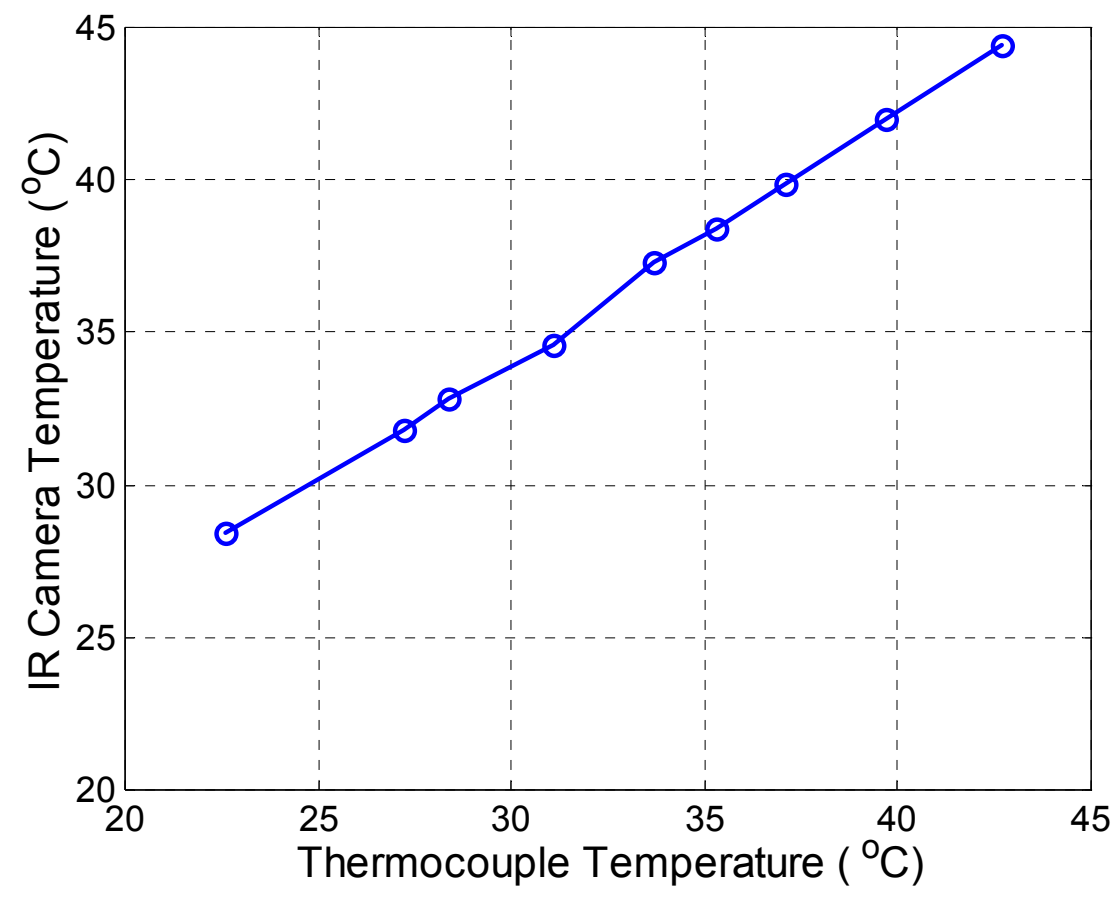

Figure 113 Temperature measured with calibrated thermocouple and IR camera.

Linear curve fits of several calibration curves indicate that the temperature difference measured by the IR camera is $80 \%$ of the temperature difference measured by the thermocouple in direct contact with the fluid. This holds true for emissivity values in the range of 0.9 to 1.0 .

After calibrating the IR camera, the temperature of the microfluidic channel was recorded as a function of time. The transducer was initially turned off, and the substrate, fluid, and channel cap were all at room temperature. The transducer was then excited with phase-shifted, continuous wave signals to generate acoustic streaming flow in a single direction around the channel. Images showing the temperature of the channel as at different time intervals are shown in Figure 114. 


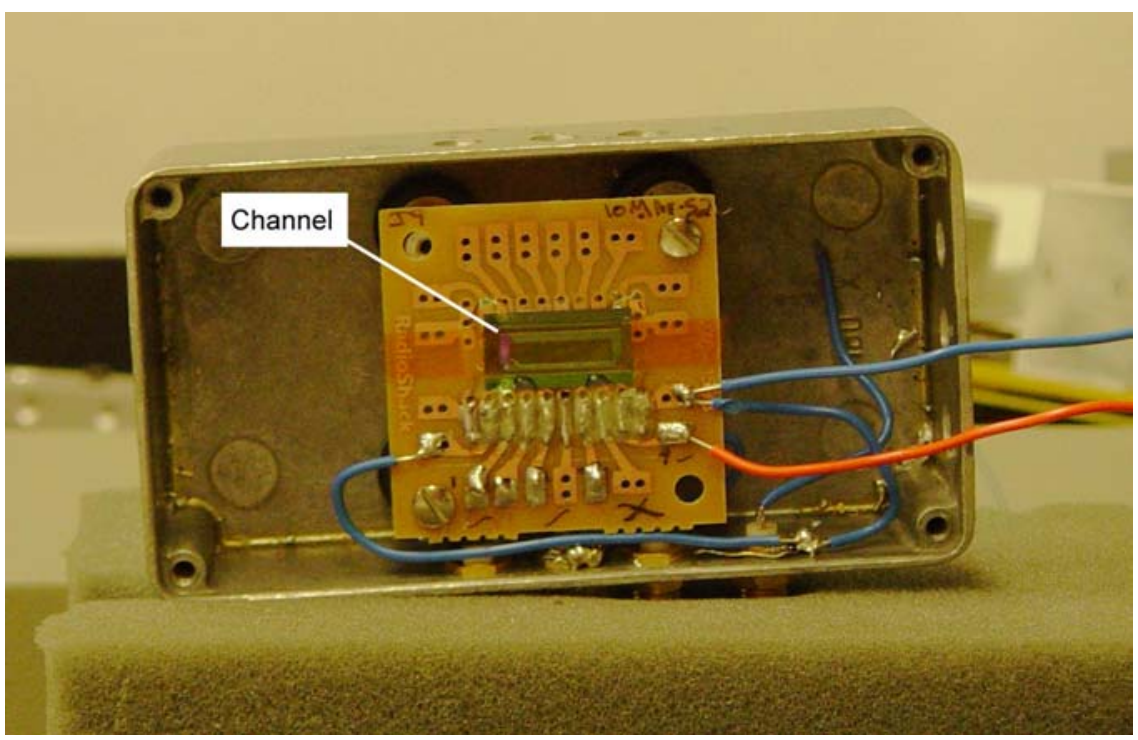

(a)
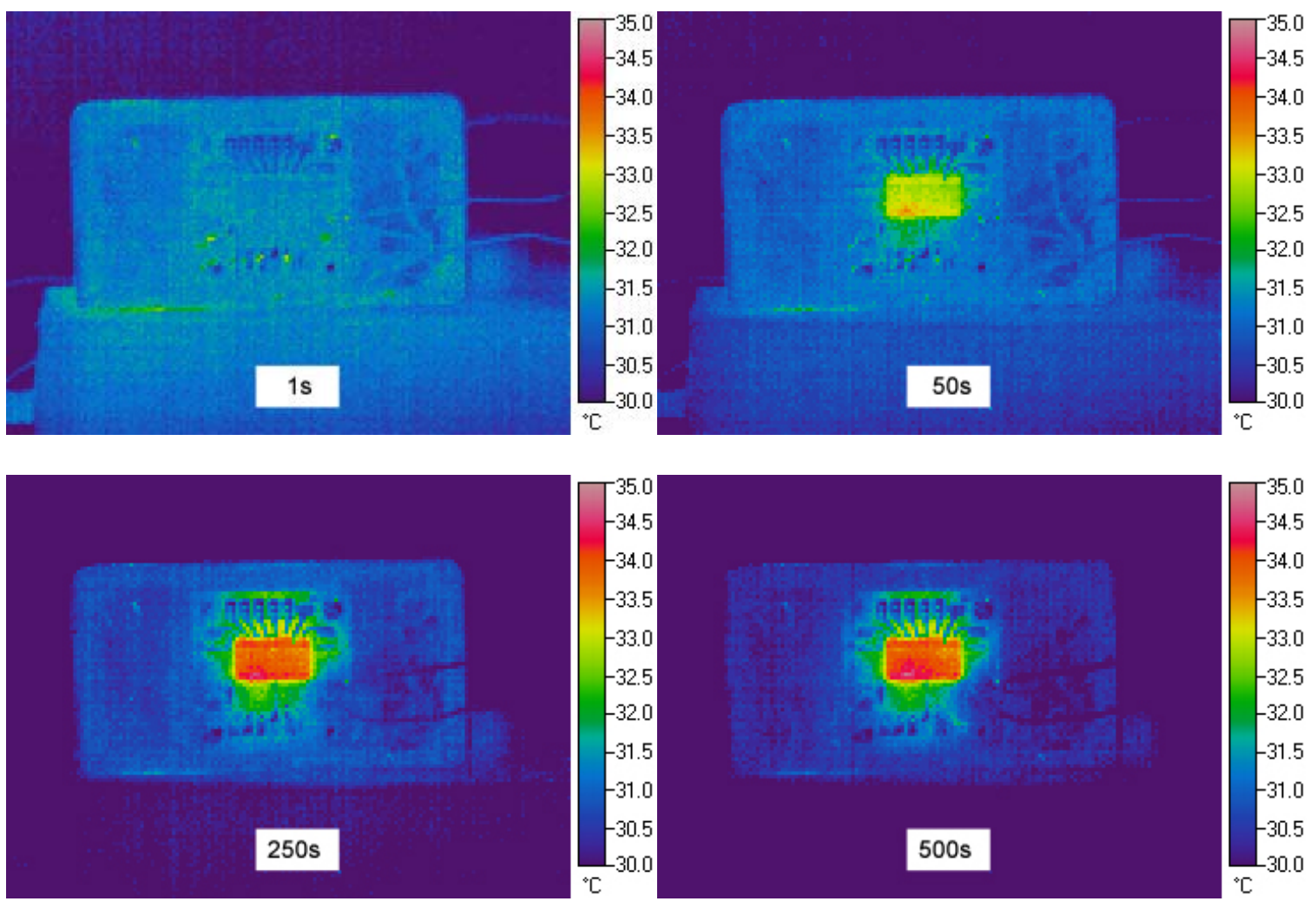

(b)

Figure 114 Generation of heat resulting form acoustic attenuation in the microchannel. (a) Picture of the device and (b) temperature distributions at various times showing acoustic heating. 
The average temperature of the channel was recorded as a function of time and corrected to indicate the temperature inside the channel. The resulting temperature history is plotted in the following figure.

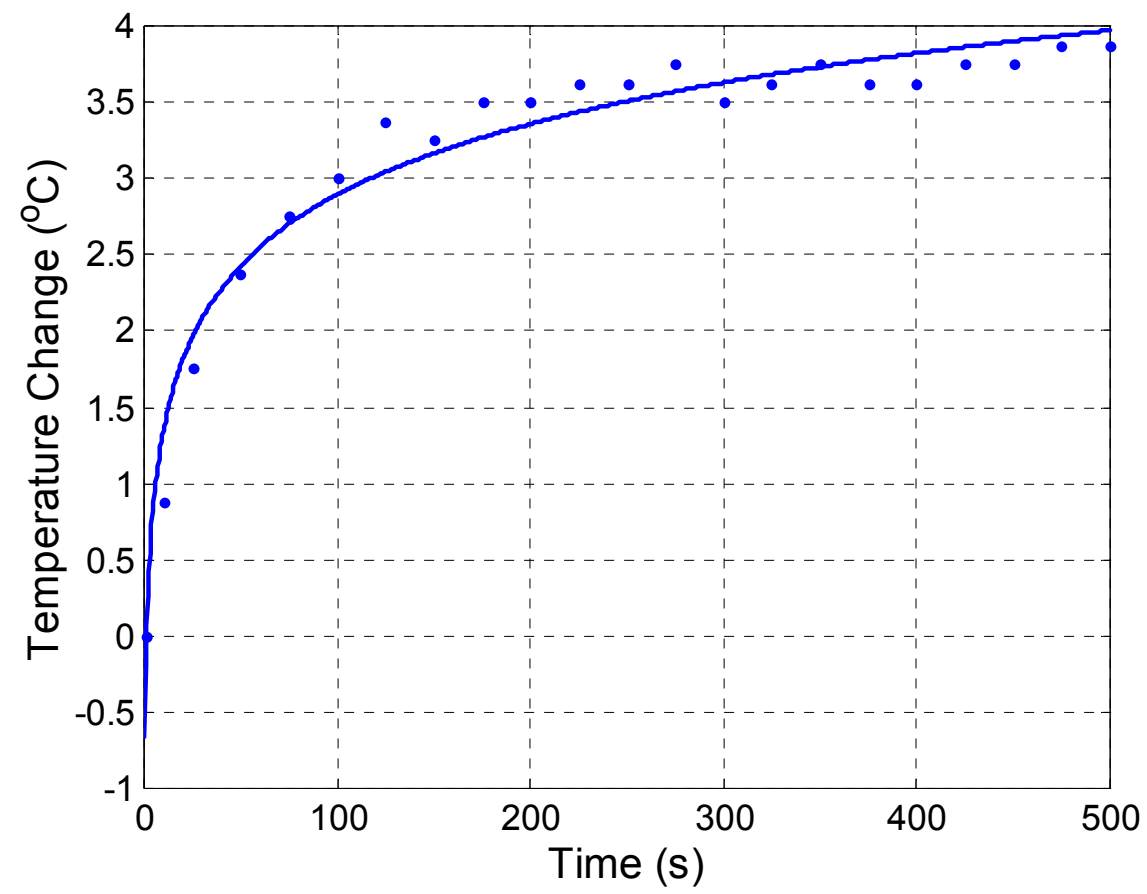

Figure 115 Average temperature of the fluid inside the channel as a function of time.

The temperature history in Figure 115 shows that the temperature rise of the channel reaches a steady state value of approximately $4^{\circ} \mathrm{C}$ after 500s. A logarithmic curve was fit to the temperature profile and is given by

$$
T(t)=0.6705 \ln (t)-0.1974 \text {. }
$$

Considering only the temperature rise of the fluid, the rate of heat generation, $\dot{Q}_{\text {Heat }}$, was calculated using the following relation,

$$
\dot{Q}_{\text {Heat }}=\rho_{0} V_{\text {Fluid }} C \frac{\Delta T}{\Delta t}
$$


where $V_{\text {Fluid }}$ is the volume of water in the channel, $C$ is the specific heat of water, $T$ is the temperature in ${ }^{\circ} \mathrm{C}$ and $t$ is the time in seconds. The time derivative of $T(t)$ was calculated and used to determine the instantaneous rate of heat generation. Since heat conduction and convection depend on a thermal gradient, these effects were assumed to be negligible during the initial heating of the fluid. The rate of heat generation was determined to be approximately $12 \mathrm{~mW}$. This amount of power is much greater than the power in the flow caused by acoustic streaming, as predicted by the analytical model.

\section{Discussion}

In this research, fluids have been pumped in microchannels using acoustic streaming. Interdigital cMUTs are used to generate guided acoustic waves which propagate in a single lateral direction which is determined by the electronic phase of the inputs. The flow rate of the fluid is readily controlled by the amplitude of the sinusoidal input voltage. The maximum flow rate generated by the pump was $410 \mathrm{~nL} / \mathrm{min}$ for a power consumption of $43 \mathrm{~mW}$. The corresponding adverse pressure gradient was calculated to be 3.4Pa. Although the experiments were carried out in de-ionized water, the fluid sensing experiments show that guided waves can be generated in a variety of fluids. Therefore, pumping is also possible in fluids other than water.

It is important to reiterate that acoustic streaming is a highly non-linear effect; thus very little of the power delivered to the fluid is coupled to the net streaming flow. This results in an extremely low thermodynamic efficiency of $5 \times 10^{-8} \%$. When compared to other types of field-induced pumps, the performance does not seem as poor. The two

main competing field-induced pump technologies are electroosmotic flow (EOF) and 
magnetohydrodynamic (MHD) pumps. Zeng et al. report electroosmotic pumping with an efficiency as high as $1.3 \%$, which is much greater than that for acoustic streaming [11]. When $2 \mathrm{kV}$ is applied to the system, the maximum pressure head and flow rate are $2 \mathrm{MPa}$ and $3.6 \mu \mathrm{L} / \mathrm{min}$. However, this approach has several disadvantages. First, voltages up to $2 \mathrm{kV}$ must be applied to generate the flow which makes integration with on-chip electronics very difficult, if not impossible. Second, the electroosmotic effect is generated in a microchannel packed with $3.5 \mu \mathrm{m}$ silica particles. This would prove problematic when attempting to pump particle-laden fluids. A limitation common to all EOF pumps is the small range of working fluids. The conductivity of the solution must be in the range of $10^{-12}$ to $10^{-6} \mathrm{~S} / \mathrm{m}$ which restricts the working fluids to organic solvents [82]. Also, all electroosmotic pumps can generate bubbles due to Joule heating which can obstruct or block the flow path [11].

Magnetohydrodynamic (MHD) pumps use the Lorentz forces acting on charged particles in a magnetic field to pump fluids. An electric current is passed through an electrically conductive fluid in the presence of a magnetic field. The magnetic field causes the charged fluid particles to move in a single direction. Lemoff et al. have demonstrated pumping of conductive fluids with an efficiency of $1.5 \times 10^{-8} \%$. The maximum pressure was $2.5 \mathrm{~Pa}$ and the maximum flow rate was $18.3 \mu \mathrm{L} / \mathrm{min}$. MHD pumps are attractive because there is a greater potential for integration when compared to membrane pumps and the flow direction can be controlled electronically. However, like EOF pumps, the working fluids must have very specific properties (conductivity and permittivity) to operate. Another major limitation arises from the fact that current is passing through the fluid. Electrolysis can form bubbles which then obstruct the flow. 
The acoustic streaming pump developed in this work has a low efficiency but has several favorable characteristics. First, there is a great potential for integration of the pump into microfluidic systems due to the surface micromachining process. The fabrication process also allows the transducers to be fabricated on top of CMOS electronics for monolithic electronics integration. Second, this acoustic streaming pump can be used to pump a wide variety of fluids. Since the flow rate and pressure head are rather low, this acoustic streaming pump will probably not be useful for high flow rate or high pressure applications. These are best served by mechanical membrane pumps. The acoustic pump is much more suited to manipulating small amounts of fluid in microfluidic system.

The performance of this acoustic streaming pump can be improved in several ways. The first and most obvious way to increase the flow rate is to input more power to the fluid. This can be accomplished by using a larger number of finger pairs in the transducer. This generates a more directional wave and couples more power into waves propagating in a single lateral direction. Since the streaming field is dependent upon the amplitude of the propagating acoustic wave, an increase in the amplitude of the wave improves the pumping performance.

Since the streaming flow is dependent on the square of the excitation frequency, increasing the operating frequency can drastically improve the performance of the pump. The maximum frequency limit is determined by the fabrication process. One must be able to fit membranes with the desired resonant frequency into ever decreasing length since the wavelength decreases with increasing frequency. The minimum feature size of the current process is approximately $2 \mu \mathrm{m}$. Since membranes with the desired resonant 
frequency must be located at $\lambda / 4$ locations, the maximum frequency attainable with the current process is in the range of $40-50 \mathrm{MHz}$.

However, interdigital cMUTs designed for operation at $10-20 \mathrm{MHz}$ can be used at odd harmonics of the design frequency. For example, an interdigital cMUT designed for operation at $10 \mathrm{MHz}$ has a periodicity of $146 \mu \mathrm{m}$. The membranes of a single finger are located at $\lambda / 4$ locations and are driven $90^{\circ}$ out of phase. This transducer can also generate directional guided waves at $30 \mathrm{MHz}$. Now the membranes of a single finger are located at $3 \lambda / 4$ locations instead of $\lambda / 4$. Again, a highly directional traveling wave is generated when the membranes are driven $90^{\circ}$ out of phase. Similarly, devices designed for operation at $20 \mathrm{MHz}$ could operate at $60 \mathrm{MHz}$, and devices for $40 \mathrm{MHz}$ could operate at $80 \mathrm{MHz}$. Increasing the operating frequency from 10 to $80 \mathrm{MHz}$ would improve the pumping performance by $36 \mathrm{~dB}$, which is significant. 


\section{CHAPTER 9}

\section{CONCLUSION}

Through the course of this research, capacitive micromachined ultrasonic transducers (cMUTs) have been developed for sensing and actuation applications in fluidic environments. The membranes of the cMUTs are arranged into an interdigital configuration to generate unidirectional guided waves in liquid half-spaces and microchannels. These guided acoustic modes have the majority of their energy confined to the liquid and are thus sensitive to changes in flow and fluid properties. When high amplitude guided acoustic waves are excited, the non-linear acoustic effect of acoustic streaming causes a net motion of the liquid in a microchannel in a controllable direction.

To predict the performance of the interdigital cMUT arrays, coupled-field finite element models were developed which accurately model the acoustic fields in both the solid substrate and the liquid. The models capture effects such as Scholte wave generation in liquid half-spaces and guided mode propagation in liquid-filled microchannels. The models were experimentally verified and used to develop an understanding of the parameters that affect the directionality and efficiency of interdigital cMUTs. The directionality of the guided waves and the efficiency in generating these waves were found to increase as the number of fingers was increased. The directionality, or ratio of power in the forward propagating Scholte wave to the backward propagating wave, for a 10-finger interdigital cMUT in a fluid half-space was found to be nearly $16 \mathrm{~dB}$. The efficiency was found to be strongly dependent on how well the transducer periodicity matches the acoustic wavelength of the desired mode. When the transducer 
pitch was $98.5 \%$ of the Scholte wavelength, the efficiency was found to be $14 \%$ below the maximum. This demonstrates that the frequency of operation should be tuned to deliver the maximum amount of power to the fluid. The models were also used to determine where the acoustic energy flowed in the system. When transducers are excited in a liquid half-space, approximately $50 \%$ of the acoustic power input to the system goes into a Scholte wave propagating in a single lateral direction. The remaining $50 \%$ of the power is radiated into the liquid half-space as a bulk pressure wave. When transducers are incorporated into a microchannel, $80 \%$ of the total acoustic power propagates in a single lateral direction which makes the transducers attractive for liquid actuation applications. The increase in directional power is attributed to the fact that the bulk waves radiated by the array can only generate standing waves in the channel or propagate away from the transducer; they cannot propagate away from the substrate.

After designing the transducers with finite element models, they were fabricated using a surface-micromachining process. To fully realize the potential of the surface micromachining process, the transducers need to be capable of being integrated with other sensors, microfluidic networks, and signal processing electronics. To this end, a PECVD-based fabrication process was developed which has a maximum processing temperature of $250^{\circ} \mathrm{C}$. The process is capable of producing cMUTs with small gaps, down to $500 \AA$, and no performance trade-offs as compared to other cMUT fabrication processes. The process provides the potential for cMUTs to be post-processed over CMOS electronics and integrated with a variety of microfluidic systems.

The fabrication process was used to produce the first unidirectional, interdigital cMUT arrays. Although the transducers for this research were designed for microfluidic 
applications, interdigital cMUT arrays have the potential to compete with interdigital piezoelectric transducers for SAW applications. CMUT arrays are superior to piezoelectric transducers since they can be fabricated in batches with well established surface-micromachining techniques and integrated with signal processing electronics. Additionally, cMUTs have superior bandwidth and a significantly lower insertion loss as compared to piezoelectric transducers [17].

The interdigital cMUTs were used demonstrate the capabilities of a new type of acoustic liquid sensor based on Scholte and guided wave propagation. Guided modes generated in microchannels were used to sense changes in liquid sound speed and flow rate. The minimum detectable change in fluid sound speed was $0.25 \mathrm{~m} / \mathrm{s}$ while the minimum detectable change in flow rate was $1 \mathrm{~mL} / \mathrm{min}$. In fluid half-spaces, the unique nature of the Scholte wave allows one to measure the visco-elastic properties of the surrounding liquid using a pair of transducers on a single substrate. A transducer immersed in a water half-space was demonstrated to sense changes in temperature as small as $0.1^{\circ} \mathrm{C}$. This "dipstick" measurement represents an improvement over existing techniques since both transducers are on the same substrate and separated by a precise distance.

Finally, an acoustic streaming-based micropump was developed which can be easily integrated into microfluidic systems and pump a variety of liquids. The interdigital cMUTs generate highly directional guided waves in microchannels which in turn generate acoustic streaming flow. Since acoustic streaming is a highly non-linear effect, only a small portion of the acoustic energy delivered to the fluid is transferred to fluid flow. Hence, the efficiency of this pump is quite low, approximately $5 \times 10^{-8} \%$. For a 
power consumption of $43 \mathrm{~mW}$, a flow rate of $410 \mathrm{~nL} / \mathrm{min}$ was generated against a pressure of 3.4Pa. The pumping performance can be improved significantly by delivering more acoustic power to the fluid and by increasing the operating frequency of the interdigital cMUTs. Although the efficiency and pressure head are low, these transducers can be useful for precisely manipulating small amounts of fluid around microfluidic networks.

\section{$\underline{\text { Recommendations }}$}

In this research, the foundation for a new type of micromachined acoustic sensor and actuator has been established. However, several items should be addressed before these transducers find widespread application. Although the cMUT fabrication process should be CMOS compatible, steps must be taken before electronics integration can be realized. As a first step, one should demonstrate that the fabrication process does not significantly alter the performance of CMOS electronics. This experiment can be carried out by taking a working CMOS chip through the steps of the cMUT process and documenting its performance before and after. The next step towards electronics integration would be to develop a process steps which prepares the surface of the electronics chip for cMUT fabrication. The important steps are electrical passivation and planarization of the surface. An approach similar to the one used by Texas Instruments for their Digital Mirror Device (DMD) would be a useful benchmark [83].

For the guided acoustic wave flow and liquid sensor to be commercially viable, further characterization and rigorous calibration are required. Minor alterations to the transducer design could also be made to improve the minimum detectable change in sound speed and flow rate. The signal-to-noise ratio of $25 \mathrm{~dB}$ can be improved by 
increasing the number of finger pairs in the transmitter. This will help decrease the jitter in the phase velocity measurement and thus increase the measurement accuracy. Shielding from electromagnetic noise would also improve the sensor performance. The performance of the interdigital cMUT fluid sensor can be optimized by monolithically integrating the transducer with drive and sensing electronics. This would drastically reduce the parasitic capacitance and thus increase the signal-to-noise ratio.

The acoustic streaming pump should be pursued further, but a suitable application should be found. With an application in mind, one can select the number of fingers and operating frequency to achieve the performance specifications. The pumping performance of the current pump can be improved by coupling more power to the fluid using a greater number of fingers and by operating at higher frequencies. Additionally, research on integrating the pump with other sensors in a microfluidic system should be pursued. 


\section{APPENDIX 1}

\section{CODE FOR ANALYTICAL MODEL}

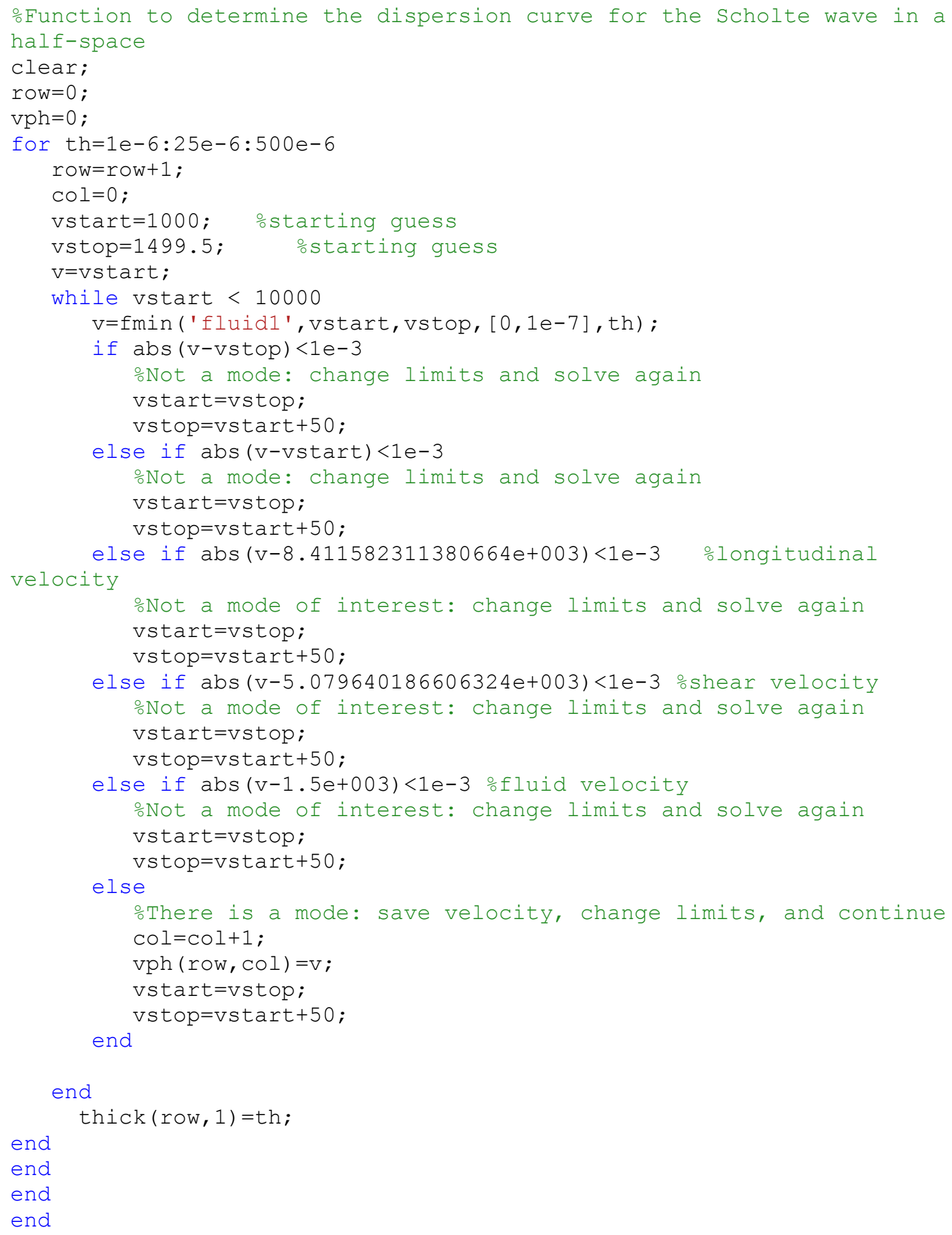




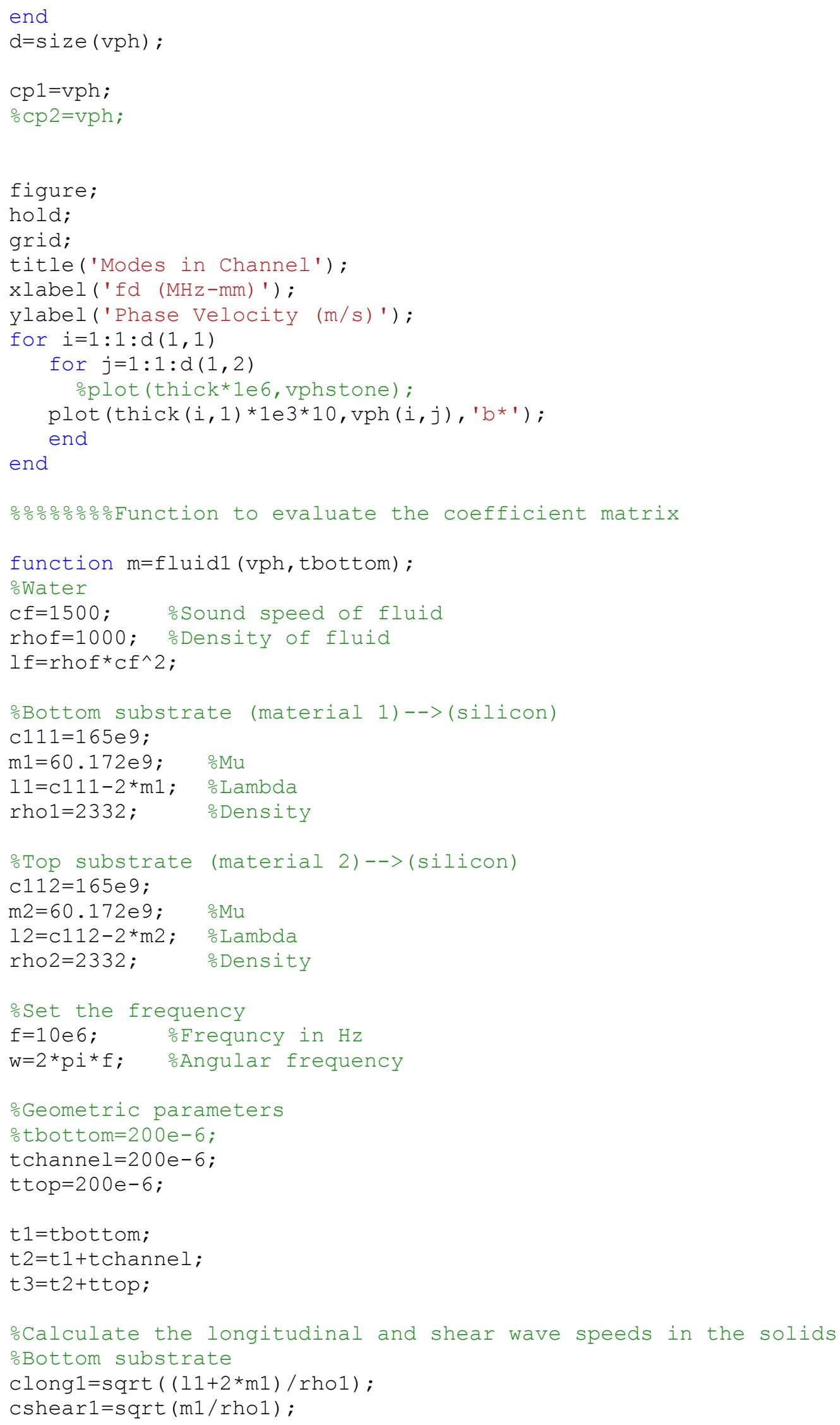




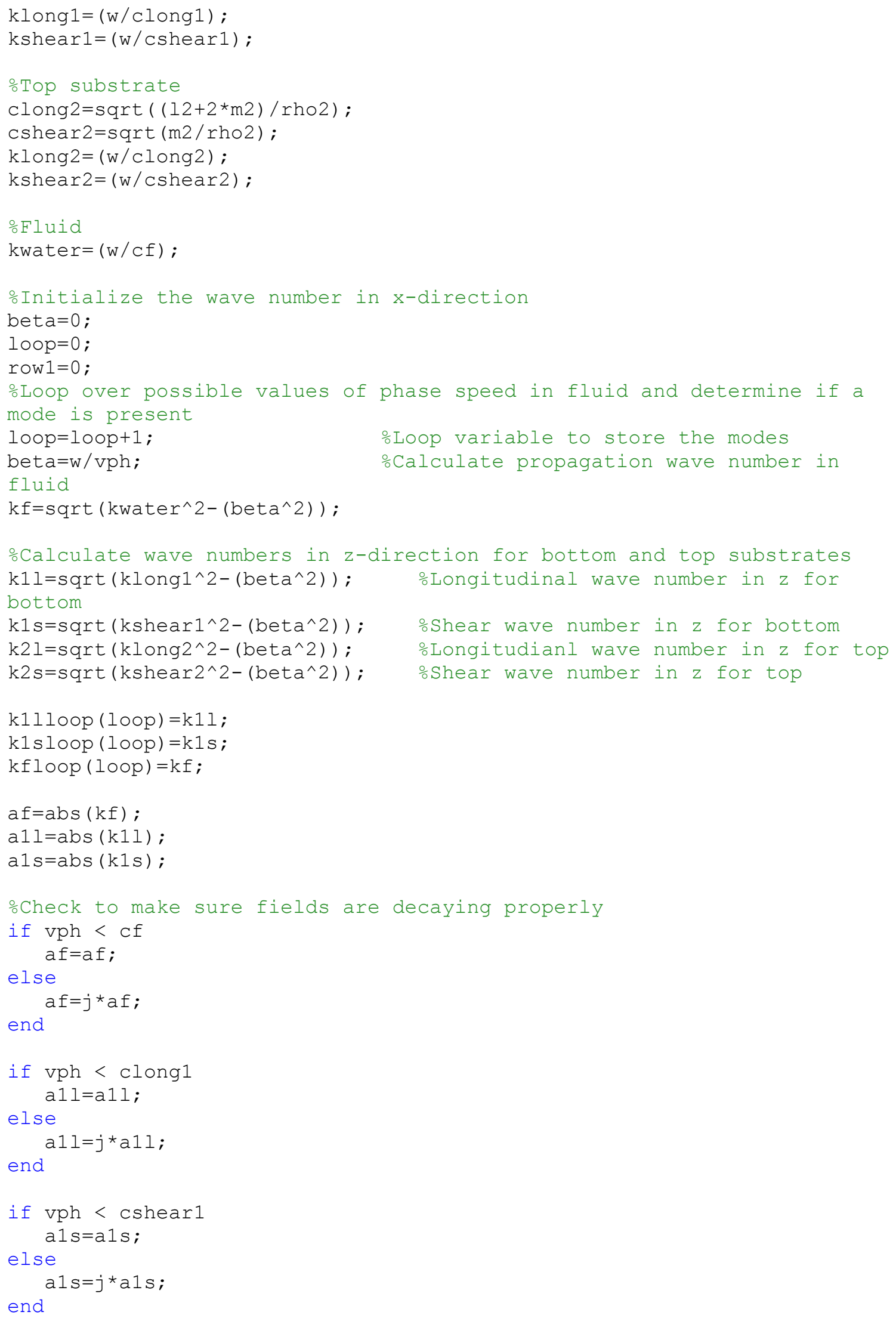




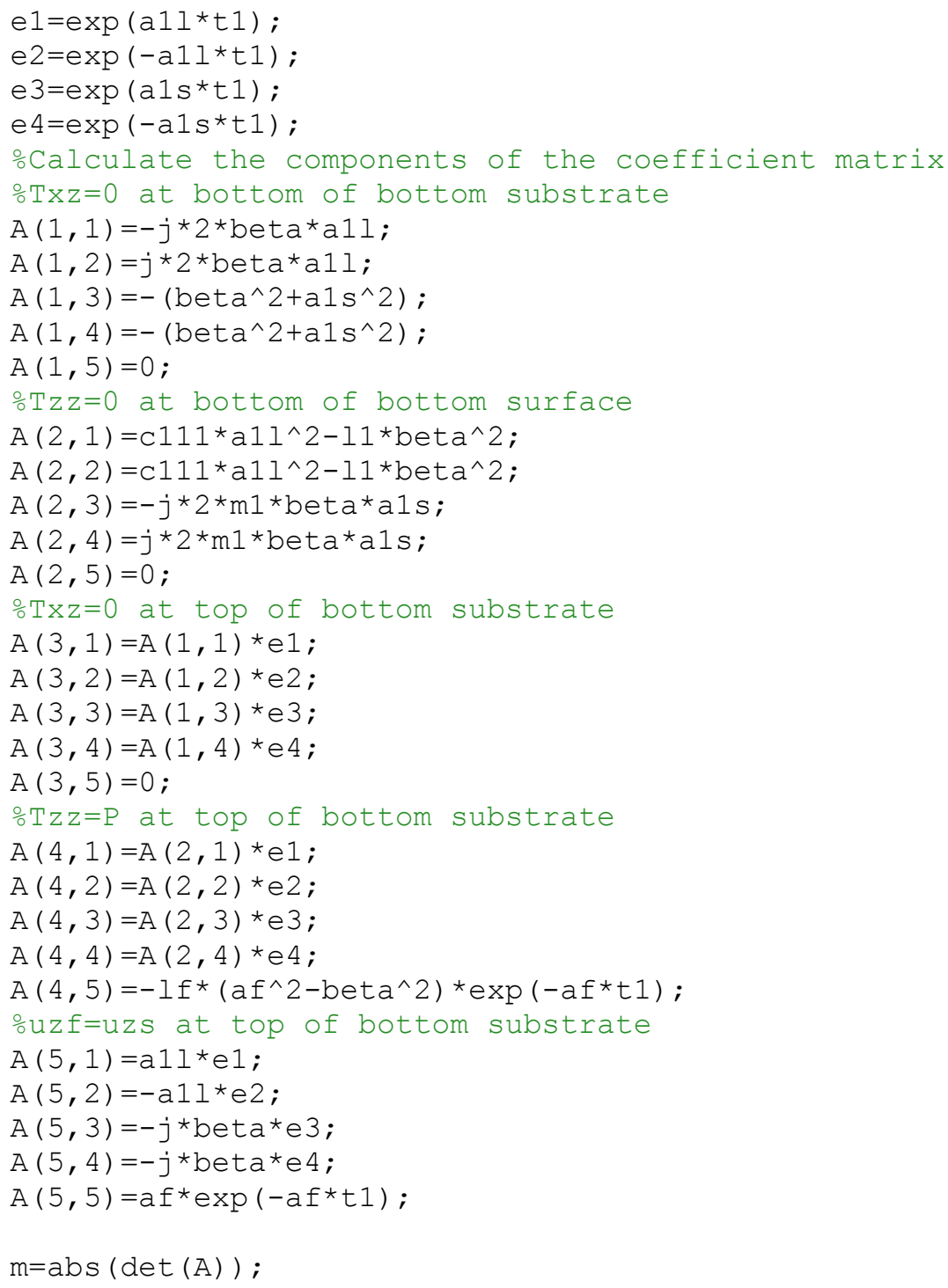




\section{APPENDIX 2}

\section{CODE FOR FINITE ELEMENT MODELS}

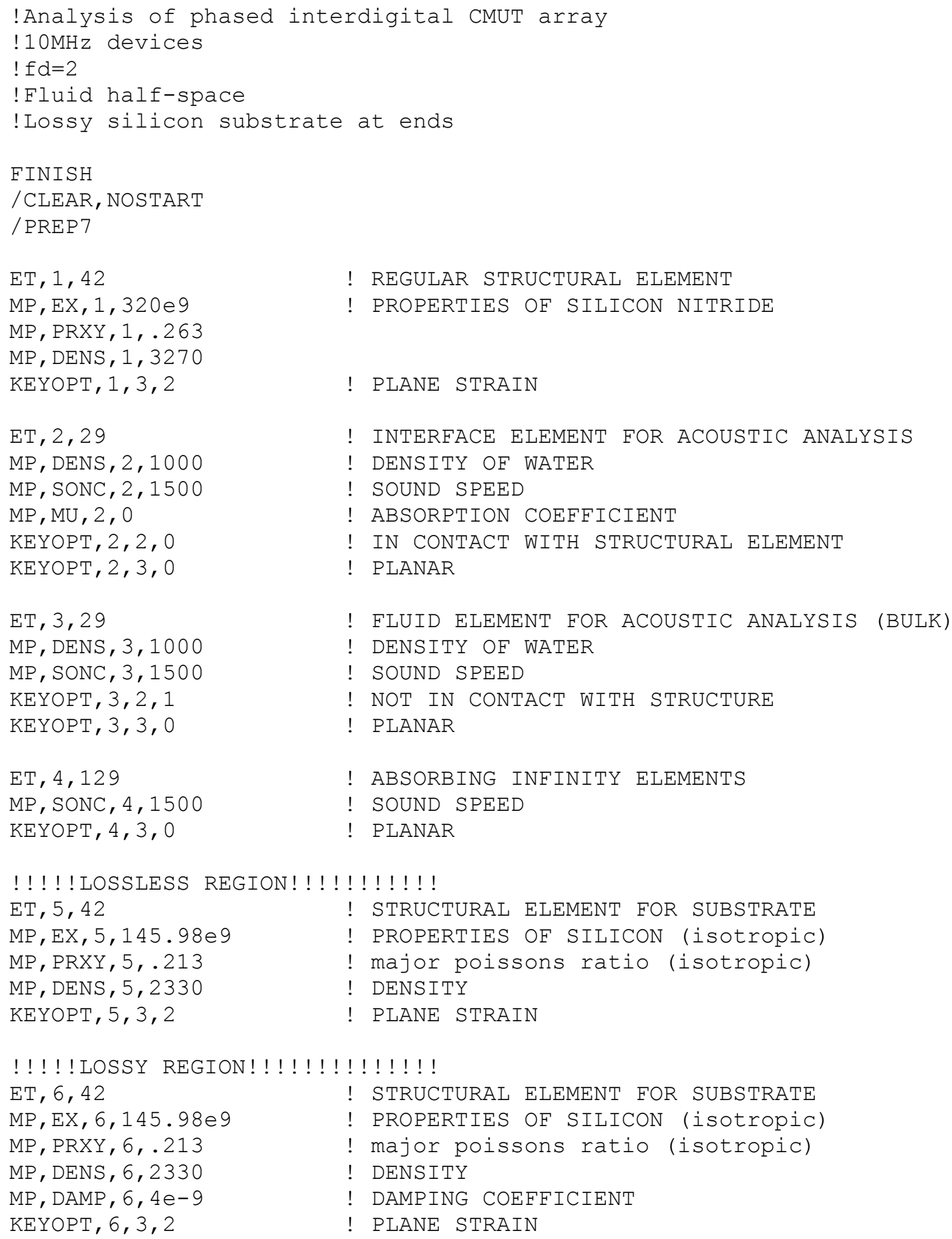




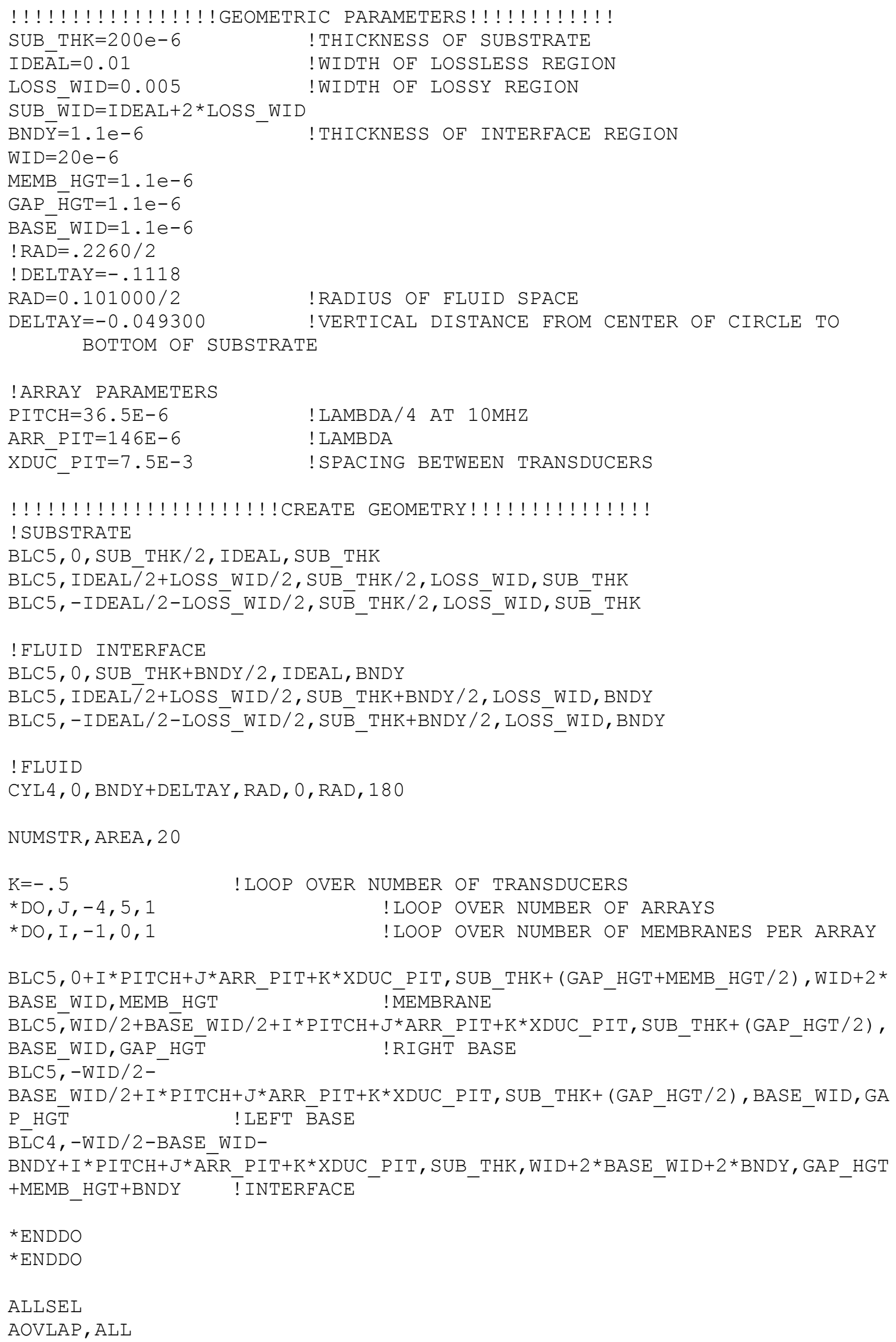




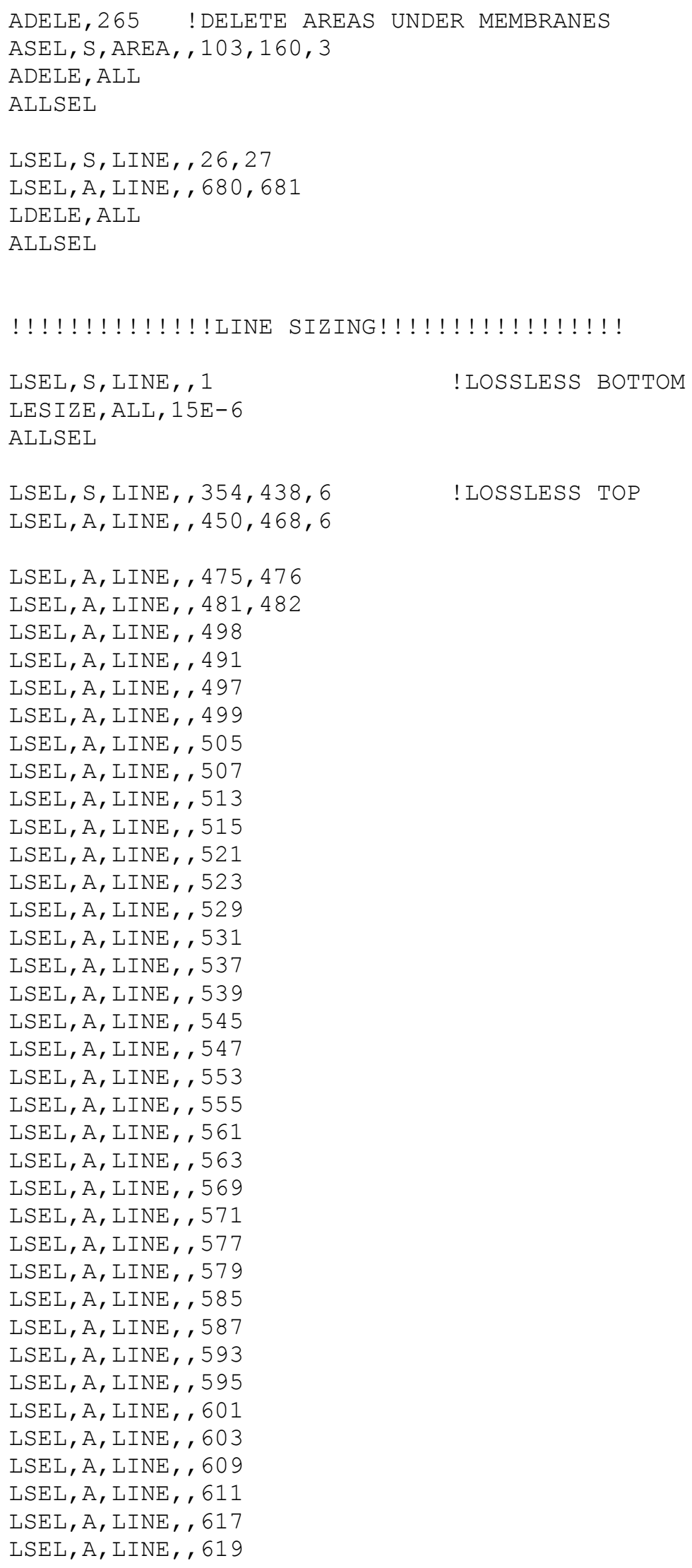




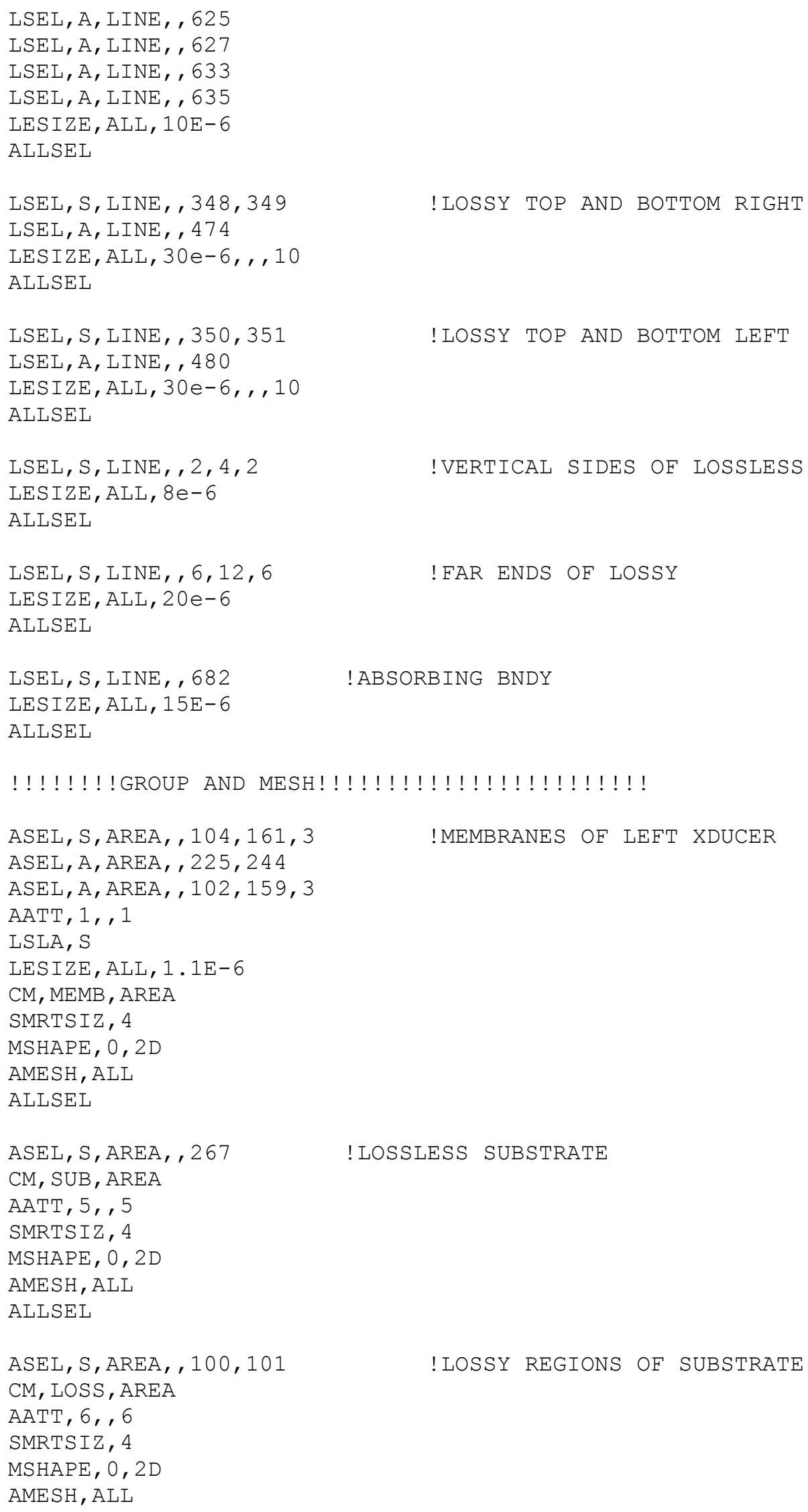




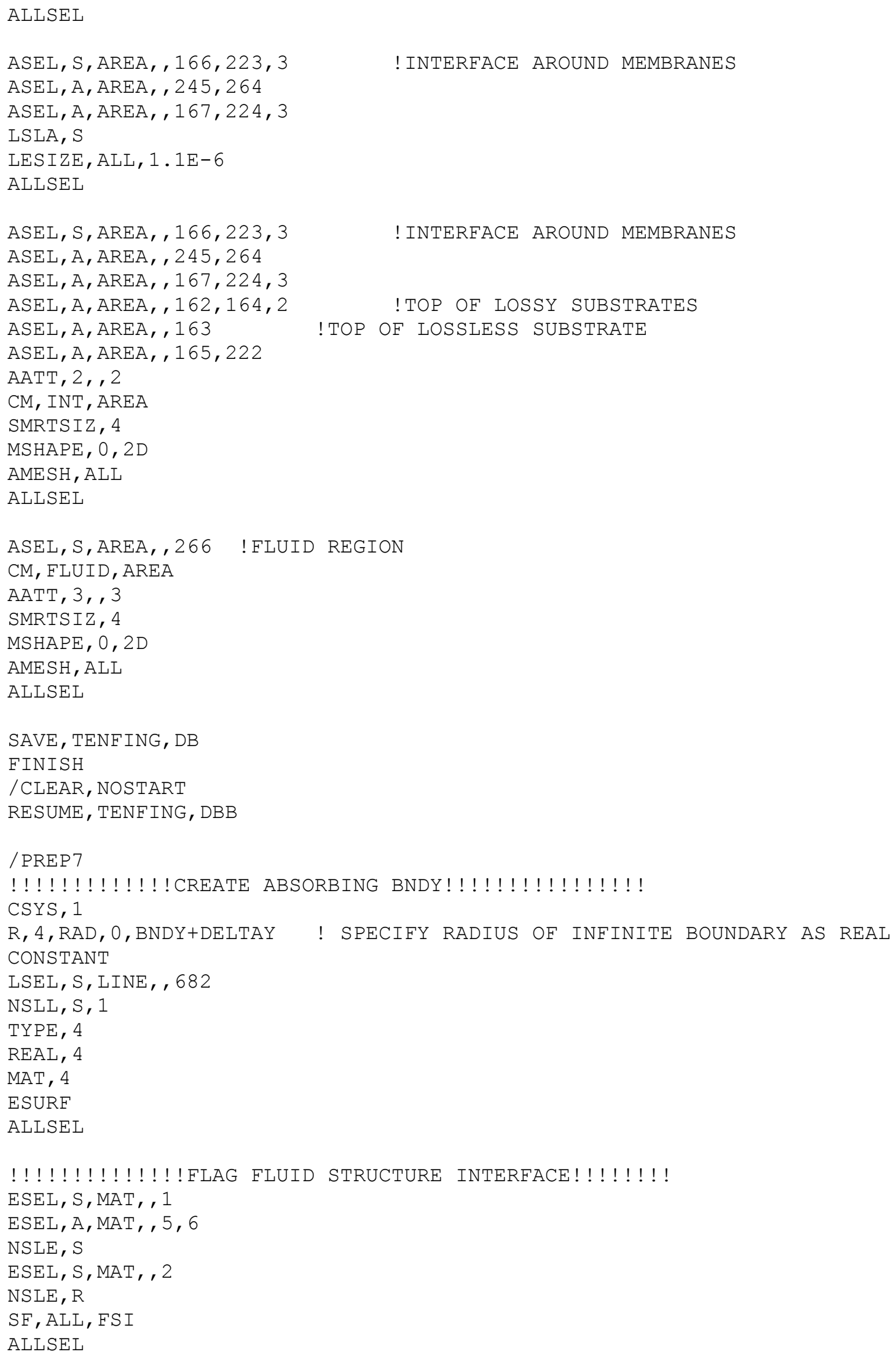




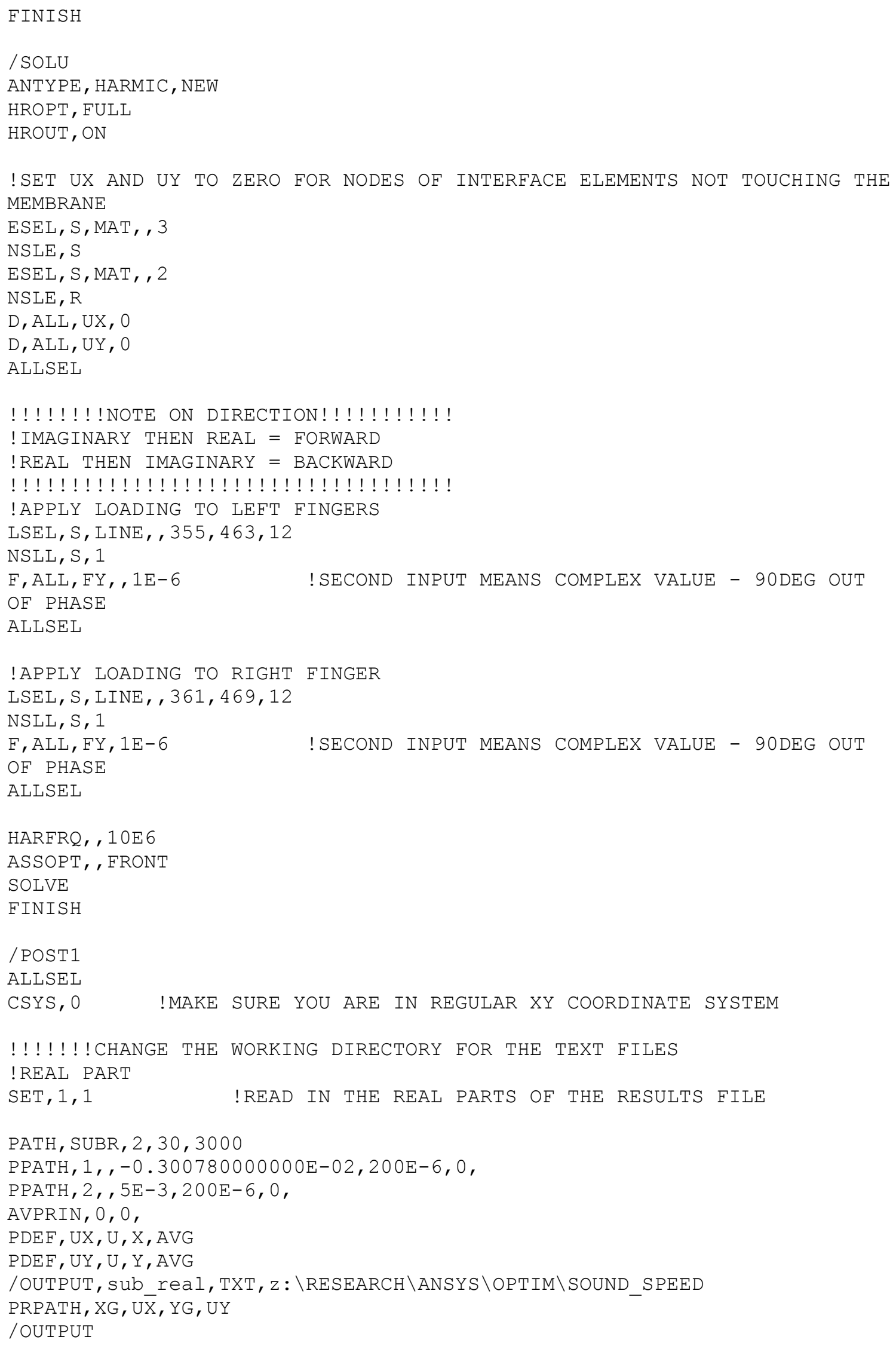


PATH, MEMB100A, 2, 30, 200

PPATH $, 1,0.004,200 \mathrm{E}-6,0$

PPATH $, 2,0.004,0.1001 \mathrm{E}-02,0$

PDEF, PGY, PG, Y, AVG

$\mathrm{PDEF}, \mathrm{PGX}, \mathrm{PG}, \mathrm{X}, \mathrm{AVG}$

PDEF, PRESS, PRES, , AVG

AVPRIN, 0,0 ,

/OUTPUT, POWER_RIGHT_REAL, TXT, z: \RESEARCH \ANSYS $\backslash O P T I M \backslash S O U N D$ SPEED

PRPATH, YG, PRES S, PGX, PGY

/OUTPUT

PATH , MEMB10A, 2, 30, 1000

PPATH $, 1,-0.43816 \mathrm{E}-2,0.2022 \mathrm{E}-3,0$

PPATH $, 2,-0.31549 \mathrm{E}-2,0.2022 \mathrm{E}-3,0$

$\mathrm{PDEF}, \mathrm{UY}, \mathrm{U}, \mathrm{Y}, \mathrm{AVG}$

PDEF, PRESS, PRES, , AVG

AVPRIN, 0,0 ,

/OUTPUT, POWER_MEMB_REAL, TXT, z: \RESEARCH \ANSYS \OPTIM \SOUND_SPEED

PRPATH, XG, YG, $\mathrm{PRESS}, \mathrm{UY}$

/OUTPUT

! IMAGINARY PART

$\mathrm{SET}, 1,1,1$

! READ IN THE REAL PARTS OF THE RESULTS FILE

PATH, SUBI , $2,30,3000$

PPATH, 1, , $-0.300780000000 \mathrm{E}-02,200 \mathrm{E}-6,0$,

PPATH , 2, 5E-3, 200E-6, 0 ,

AVPRIN, 0, 0 ,

$\mathrm{PDEF}, \mathrm{UX}, \mathrm{U}, \mathrm{X}, \mathrm{AVG}$

$\mathrm{PDEF}, \mathrm{UY}, \mathrm{U}, \mathrm{Y}, \mathrm{AVG}$

/OUTPUT, sub_imag, TXT, z: \RESEARCH \ANSYS $\backslash O P T I M \backslash S O U N D$ SPEED

PRPATH, XG, UX, YG, UY

/OUTPUT

PATH , MEMB100A, 2, 30, 200

PPATH $, 1,0.004,200 \mathrm{E}-6,0$

PPATH , 2, $0.004,0.1001 \mathrm{E}-02,0$

$\mathrm{PDEF}, \mathrm{PGY}, \mathrm{PG}, \mathrm{Y}, \mathrm{AVG}$

$\mathrm{PDEF}, \mathrm{PGX}, \mathrm{PG}, \mathrm{X}, \mathrm{AVG}$

PDEF, PRESS, PRES, , AVG

AVPRIN, 0,0 ,

/OUTPUT, POWER RIGHT IMAG, TXT, z: \RESEARCH \ANSYS \OPTIM \SOUND SPEED

PRPATH, YG, PRE $\bar{S} S, P G \bar{Y}, \mathrm{PGY}$

/OUTPUT

PATH, MEMB10A, 2, 30, 1000

PPATH , 1, , $0.43816 \mathrm{E}-2,0.2022 \mathrm{E}-3,0$

PPATH , $2,-0.31549 \mathrm{E}-2,0.2022 \mathrm{E}-3,0$

$\mathrm{PDEF}, \mathrm{UY}, \mathrm{U}, \mathrm{Y}, \mathrm{AVG}$

PDEF, PRESS, PRES, , AVG

AVPRIN, 0,0 ,

/OUTPUT, POWER_MEMB_IMAG, TXT, z: \RESEARCH \ANSYS \OPTIM \SOUND_SPEED

PRPATH, XG, YG, $\mathrm{PRESS}, \mathrm{UY}$

/OUTPUT

FINISH 


\section{APPENDIX 3}

\section{DETAILED CMUT FABRICATION PROCESS}

\section{cMUT Process Flow}

\section{Chromium Sacrificial Layer}

1. Deposit chromium sacrificial layer on low resistivity silicon wafers
a. CVC DC Sputterer
b. Depostion Rate $=\sim 2.5 \AA / \mathrm{s}$
c. Thickness $=1500 \AA$

2. Pattern chrome sacrificial layer

a. Use Shipley 1813 photoresist

i. Dehydrate on hotplate for $90 \mathrm{~s}$

ii. Blow of with nitrogen

iii. Cover most of wafer with resist

iv. Spin: 4000rpm, 1000rpm/s ramp, 60s

v. Soft bake on hotplate at $115 \mathrm{C}$ for $4 \mathrm{~min}$

vi. Expose on MA-6 for $6 \mathrm{~s}$ on $\mathrm{CI} 2\left(\mathrm{I}=20 \mathrm{~mW} / \mathrm{cm}^{2}\right)$ using vacuum contact

vii. Develop in MF 319 for 60 s; rinse with DI; dry with nitrogen

viii. Hardbake in oven at $120^{\circ} \mathrm{C}$ for $15-20 \mathrm{~min}$

b. Pattern chrome using Cyantek Chrome Mask Etchant

i. Immerse and agitate for 30-40s; watch for subtle color change

ii. Rinse well

c. Strip PR in acetone. After removing bulk of resist with spray, immerse in $1000 \mathrm{~mL}$ beaker with $\sim 200 \mathrm{~mL}$ of acetone. Place in ultrasonic bath for $5-10 \mathrm{~min}$ to remove all the residue.

d. Do NOT use the Gastronics Asher for the chromium; it will all be removed

3. Deposit first layer of silicon nitride for the membranes

a. Unaxis PECVD

i. Chamber should be clean before starting process; if flakes are falling off, vacuum out using metal wand and run cleaning process

b. Recipe: CMUTNITR

i. Temperature: $250^{\circ} \mathrm{C}$

ii. Stress: $30-40 \mathrm{MPa}$ Tensile

iii. Dep Rate: $82 \AA / \mathrm{min}$

iv. Target thickness $=4000 \AA$

c. Rotate wafers $180^{\circ}$ halfway through deposition to improve uniformity 
4. Open up windows to the ground electrode

a. Lithography with 1813

b. Etch down to silicon using Left Chamber of the ICP

i. Recipe: jjm_nitr.bch (uses $\mathrm{CF}_{4}$ )

ii. Rate: $\sim 2500 \AA / \mathrm{min}$; check with test wafer using Nanospec (low stress nitride recipe)

iii. Over-etch by $25 \%$

c. Strip PR as before

d. Run wafers through Process A (60s clean with heat) on the Gastronics Asher

i. Necessary to remove organic residue before depositing metal

ii. Makes the metal come out smooth and shiny

5. Deposit top electrode: aluminum and chrome

a. CVC DC Sputterer

b. Dep. Rates

i. Aluminum: $5-6 \AA / \mathrm{s}$

ii. Chrome: $\sim 2-3 \AA / \mathrm{s}$

c. Deposit $1200 \AA$ of aluminum then $300 \AA$ of chrome

6. Pattern top electrode

a. Shipley 1813 lithography recipe

i. Use hard contact mode

ii. Alignment gap of $20 \mu \mathrm{m}$

iii. Use "alignment check" to check alignment before exposure

b. Chrome etchant for 10-20s; watch for color change

i. Rinse very well in DI

c. Aluminum Etchant Type A, heated to $50^{\circ} \mathrm{C}$ on the hotplate

i. Immerse wafer, swirl around

ii. Remove $10-15$ s after aluminum clears

iii. Rinse well

d. Strip PR as before using acetone in ultrasonic bath as final step

7. Second membrane deposition
a. Unaxis PECVD
b. CMUTNITR
c. Thickness $=4000 \AA$

8. Open up etch holes in nitride

a. Lithography using $1827(\sim 2.3 \mu \mathrm{m}$ of resist $)$

i. Blow of with nitrogen

ii. Cover entire wafer with resist

iii. Spin: 4000rpm, 1000rpm/s ramp, 60s

iv. Soft bake on hotplate at $115 \mathrm{C}$ for $4 \mathrm{~min}$ 
v. Expose on MA-6 for $4 \mathrm{~s}$ on CI2 using hard contact with align gap of $20 \mu \mathrm{m}$; alignment was hard b/c of dark field mask

vi. Develop in MF 319 for 60s; rinse with DI; dry with nitrogen

vii. Hardbake in oven at $120^{\circ} \mathrm{C}$ for $15-20 \mathrm{~min}$

b. Etch with RIE through nitride

i. Etch rate: $\sim 600 \AA / \mathrm{min}$

ii. Rotate $180^{\circ}$ halfway through to improve uniformity

iii. Overetch by $25-50 \%$; ok since chrome acts as etch stop

9. Release membranes

a. Before stripping PR, immerse wafer in chrome etchant for $10 \mathrm{~min}$ to make sure that etch holes are open

b. Strip PR in solvents

c. Immerse wafer in chrome etchant heated to $50^{\circ} \mathrm{C}$ to increase etch rate

d. After released, rinse wafer well in DI

e. Clean wafer using solvents, including acetone-ultrasound process

10. Seal membranes

a. Unaxis PECVD

b. Deposit $4500-6000 \AA$ to make sure membranes are sealed

11. Thin membranes to desired thickness

a. Lithography with 1813

b. Etch back using left chamber of ICP

i. Recipe: jjm_nitr.bch

ii. Check thickness before starting etch using the Nanospec

iii. Run for half of expected time; check thickness

iv. Adjust rate and finish etch

v. Desired thickness is $8000 \AA$

c. Strip wafer using solvents

12. Open up holes through passivation layer to bond pads

a. 1827 Lithography

b. Etch with RIE (don't want metal in ICP)

i. Etch through $4000 \AA$ of nitiride

ii. Overetch by $25 \%$ but not much more

c. Strip PR

13. Deposit fresh aluminum on bond pads to improve wire bonding

a. CVC DC Sputterer

b. $1000 \AA$ of aluminum 
c. Pattern using negative resist and mask used to etch down to bond pads

i. NR9-8000P

ii. Follow recipe from Futurex

d. Strip PR in solvents

14. Drill vias through the silicon

a. Lithography with AZ 4620 (yields $23 \mu \mathrm{m}$ )

i. Do NOT do dehydration bake; it will cause the metal to oxidize!

ii. Spin: $600 \mathrm{rpm}, 500 \mathrm{rpm} / \mathrm{s}, 40 \mathrm{~s}$

iii. Softbake for $10 \mathrm{~min}$ at $100^{\circ} \mathrm{C}$

iv. Expose on MA-6

v. Develop for $2 \mathrm{~min}$ in AZ400K

1. 2parts water, 1 part developer

vi. Hardbake for $10 \mathrm{~min}$ at $100^{\circ} \mathrm{C}$

b. Mount cMUT wafer to carrier wafer using 3 drops of 1827

i. Put PR on carrier wafer

ii. Move device wafer relative to carrier to distribute resist

iii. Bake for $15-20 \mathrm{~min}$ on hotplate at $100^{\circ} \mathrm{C}$

c. Run wafer in right chamber of the ICP

i. Recipe: twsifsta.bch

ii. Rate: $0.48 \mu \mathrm{m} /$ cycle

iii. Takes 1200-1300cycles to get through wafer

d. Strip wafer using solvents and Asher if necessary

15. Dice wafer using dicing saw in Dr. Joshi's lab

a. Recipe: MosesSi

b. Feed: $4-5 \mathrm{~mm} / \mathrm{s}$

16. Wire bond

a. Use MEI Wire Bonder in Love Rm. 10 


\section{REFERENCES}

1. S. Radhakrishnan and A. Lal, "A Drag Force Flow Sensor for Closed-Loop Flow Control”, Proceedings of IEEE MEMS, Kyoto, pp. 307-310 (2003)

2. H. Ernst, A. Jachimowicz, and G.A. Urban, "High resolution flow characterization in Bio-MEMS", Sensors and Actuators A, 100, pp. 54-62 (2002)

3. W.L. Nyborg, in Nonlinear Acoustics, edited by M.F. Hamilton and D.T. Blackstock, Chapter 7 (1998)

4. J. Khandurina and A. Guttman, "Bioanalysis in microfluidic devices", Journal of Chromatography A, 943, pp. 159-183 (2002)

5. G.H.W. Sanders and A. Manz, "Chip-based microsystems for genomic and proteomic analyis", Trends in Analytical Chemistry, Vol. 19, No. 6, pp. 364-378 (2000)

6. B.H. Weigl, R.L. Bardell, and C.R. Cabrera, "Lab-on-a-chip for drug development", Advanced Drug Delivery Reviews, 55, pp. 349-377 (2003)

7. R. Linnemann, P. Woias, C.-D.Senfft, and J.A. Ditterich, "A Self-priming and Bubbletolerant Piezoelectric Silicon Micropump for Liquids and Gases", Proceedings of Eleventh Annual International Workshop on MEMS, IEEE, pp. 532-537 (1998)

8. W.L. Benard, H. Kahn, A. Heuer, and M.A. Huff, "Thin-film shape memory alloy actuated micropumps", IEEE Journal of MEMS, Vol. 7, No. 2, pp. 245-251 (1998)

9. D.S. Popescu, P. Lerch, C. Dunare, and D. Dascalu, "Modelling and optimization for an electrostatic actuation of a valveless micropump using a silicon buckled membrane", Proceeding of IEEE Semiconductors Conference, pp. 156-160 (1997)

10. H. Chujo, K. Matsumoto, and I. Shimoyama, "A high flow rate electro-osmotic pump with small channels in parallel”, Proceedings of IEEE MEMS, Kyoto, pp. 351-354 (2003)

11. L. Chen, J. Ma, F. Tan, Y. Guan, "Generating high-pressure sub-microliter flow rate in packed microchannel by electroosmotic force: potential application in microfluidic systems", Sensors and Actuators B, 88, pp. 260-265 (2003)

12. C.E. Bradley, J.M. Bustillo, and R.M. White, "Flow Measurements in a Micromachined Flow System with Integrated Acoustic Pumping”, Proceedings of 1995 IEEE Ultrasonics Symposium, pp.505-510 (1995)

13. J. Flood, "Ultrasonic Flowmeter Basics", Sensors Magazine, www.sensormag.com (1997)

14. T. Ritter, T.R. Shrout, and K.K. Shung, "A 30-MHz Piezo-Composite Ultrasound Array for Medical Imaging Applications”, IEEE Trans. on UFFC, Vol.49, pp. 217-230 
15. H. Jagannathan, G.G. Yaralioglu, A.S. Ergun, F.L. Degertekin and B.T. KhuriYakub, "Micro-Fluidic Channels with Integrated Ultrasonic Transducers", Proceedings of 2001 IEEE Ultrasonics Symposium, pp.859-862 (2001)

16. H.T. Soh, I. Ladabaum, A. Atalar, C.F. Quate, and B.T. Khuri-Yakub, "Silicon micromachined ultrasonic immersion transducers", Applied Physics Letters, Vol. 69, No. 24, pp. 3674-3676 (1996)

17. X.C. Jin, I.O. Oralkan, F.L. Degertekin and B.T. Khuri-Yakub, "Characterization of One-dimensional Capacitive Micromachined Ultrasonic Immersion Transducer Arrays", IEEE Trans. on UFFC, Vol. 48, pp.750-760 (2001)

18. J. McLean and F.L. Degertekin, "Capacitive Micromachined Ultrasonic Transducers with Asymmetric Membranes for Microfluidic Applications", Proceedings of 2001 IEEE Ultrasonics Symposium, pp.925-928 (2001)

19. G.G. Yaralioglu, M.H. Badi, A.S. Ergun, C.H. Cheng, F.L. Degertekin and B.T. Khuri-Yakub, "Lamb wave devices using capacitive micromachined ultrasonic transducers", Appl. Phys. Lett., Vol 78, pp.111-113 (2001)

20. M.H. Badi, G.G. Yaralioglu, A.S. Ergun, S.T. Hansen, E.J. Wong, and B.T. KhuriYakub, "Capacitive Micromachined Ultrasonic Lamb Wave Transducers Using Rectangular Membranes", IEEE Trans. on UFFC, Vol. 50, pp.1191-1203 (2003)

21. M.J. Vellekoop, A.J. Van Rhijn, G.W. Lubking, and A. Venema, "All-silicon Plate Wave Oscillator System for Sensors", Sensors and Actuators A, 25-27, pp. 699-703 (1991)

22. R. Stoneley, "Elastic Waves at the Surface of Separation of Two Solids", Proc. Of the Royal Society of London, Series A, Vol. 160, No. 738, pp.416-428 (Oct. 1, 1924)

23. J.G. Scholte, "On the Stoneley-wave equation”, Proc. Ned. Akad. Wet., Vol. 45, No. 1, pp.20-25 and 159-164 (1942)

24. S. Nasr, J. Duclos, and M. Leduc, "PVDF Transducers Generating Scholte Waves", Electronics Letters, Vol 24, No 6, pp. 309-311 (1988)

25. C. Desmet, V. Gusev, W. Lauriks, C. Glorieux, and J. Thoen, "Laser-induced thermoelastic excitation of Scholte waves", Appl. Phys. Lett., Vol 68, pp.2939-2941 (1996)

26. A. Tinel and J. Duclos, "An Alternative Method for Generating Scholte Waves", Acoustic Letters, Vol. 15, No.2, pp. 30-35 (1991)

27 W.C. Wang, P. Staecker, and R.C.M. Li, "Elastic Waves Guided by a Fluid Layer: A New Type of Ultrasonic Wave Propagation", Applied Physics Letters, Vol. 15, No. 8, pp.291-293 (1970) 
28. P.W. Staecker and W.C. Wang, "Propagation of elastic waves bound to a fluid layer between two solids", Journal of the Acoustical Society of America, Vol. 53, No. 1, pp. 65-74 (1973)

29. R. Lec, J.F. Vetelino, P. Clarke, A. Roy, and J. Turner, "Prototype Microwave Acoustic Fluid Sensors", Proc. IEEE Ultrasonics Symposium, pp.543-548 (1988)

30. A. D. Pierce, Acoustics: An Introduction to Its Physical Principles and Applications, Acoustical Society of America, Woodbury, New York, pp.220-224 (1994)

31. G.S. Kino and R.S. Wagers, "Theory of Interdigital Couples on Nonpiezoelectric Substrates", Journal of Applied Physics, Vol. 44, No. 4, pp. 1480-1488 (1973)

32. J.C. Andle and J.F. Vetelino, “Acoustic Wave Biosensors", Proc. IEEE Ultrasonics Symposium, pp.451-460 (1995)

33. W.R. Smith, H. M. Gerard, J.H. Collins, T.M. Reeder, and H.J. Shaw, "Design of Surface Wave Delay Lines with Interdigital Transducers", IEEE Trans. on Microwave Theory and Techniques, Vol. MTT-17, No. 11, pp.865-873 (1969)

34. D.P. Morgan, "Directivity of Group-Type Single Phase Unidirectional SAW Transducers", Electronics Letters, Vol. 26, No. 15, pp.1201-1203

35. J. McLean and F.L. Degertekin, "Interdigital Capacitive Micromachined Ultrasonic Transducers for Sensing and Pumping in Microfluidic Applications", Proc. International Conference on Solid-State Sensors, Actuators, and Microsystems, pp. 915-918 (2003)

36. K.F. Graff, Wave Motion in Elastic Solids, Dover, New York (1991)

37. J.D. Achenback, Wave Propagation in Elastic Solids, Elsevier, New York (1975)

38. D.T. Blackstock, Fundamentals of Physical Acoustics, Wiley \& Sons, New York, p.220 (2000)

39. J. McLean and F.L. Degertekin, "Directional Scholte Wave Generation and Detection Using Interdigital Capacitive Micromachined Ultrasonic Transducers", IEEE Trans. on UFFC, Vol. 51, No. 6, pp. 756-764, (2004)

40. M. Abromowitz and I.A. Stegun, Handbook of Mathematical Functions, Dover Publications, New York (1970)

41. A. Bozkurt, F.L. Degertekin, A. Atalar and B.T. Khuri-Yakub, "Analytic Modeling of Loss and Cross-coupling in Capacitive Micromachined Ultrasonic Transducers," Proceedings of 1998 IEEE Ultrasonics Symposium, pp. 1025-28 (1998)

42. D. Certon, N. Felix, J. Guyonvarch, O.B. Matar and F. Patat, "Characterization of transducer arrays by laser interferometry: influence of acousto-optic interactions on displacement measurements in water," Proceedings of 2001 IEEE Ultrasonics Symposium, pp. 1065-8, (2001) 
43. X. Jin, I. Ladabaum F.L. Degertekin, S. Calmes and B.T. Khuri-Yakub, "Fabrication and Characterization of Surface Micromachined Capacitive Ultrasonic Immersion Transducers", IEEE Journal of Microelectromechanical Systems, Vol. 8, No. 1, pp.100-114 (1999)

44. S. Calmes, C.H. Cheng, F.L. Degertekin, X.C. Jin, S. Ergun, and B.T. Khuri-Yakub, "Highly Integrated 2-D Capacitive Micromachined Ultrasonic Transducers", Proceedings of 1999 IEEE Ultrasonics Symposium, pp. 1163-1166 (1999)

45. C.H. Cheng, A.S. Ergun, and B.T. Khuri-Yakub, "Electrical through-wafer interconnects with sub-picofarad parasitic capacitance [MEMS packaging]," Proc. of Microelectromechanical Systems Conference, pp. 18-21, (2001)

46. E. Cianci, L. Visigalli, V. Foglietti, G. Caliano, and M. Pappalardo, "Improvements toward a reliable fabrication process for CMUT", Microelectronic Engineering, 67-68, pp.602-608 (2003)

47. R.A. Noble, A.R.D. Jones, T.J. Robertson, D.A. Hutchins, and D.R. Billson, "Novel, Wide Bandwidth, Micromachined Ultrasonic Transducers", IEEE Trans. on UFFC, Vol. 48, No. 6, pp.1495-1507 (2001)

48. Y. Huang, A.S. Ergun, E. Hæggström, M.H. Badi, and B.T. Khuri-Yakub, "Fabricating Capacitive Micromachined Ultrasonic Transducers with Wafer-Bonding Technology", IEEE Journal of Microelectromechanical Systems, Vol. 12, No. 2, pp.128-137 (2003)

49. P.-C. Eccardt, K. Niederer, T. Scheiter, and C. Hierold, "Surface micromachined ultrasonic transducers in CMOS technology", Proceedings of 1996 IEEE Ultrasonics Symposium, pp. 959-962 (1996)

50. J.G. Knight, J. McLean, and F.L. Degertekin, "Low Temperature Fabrication of Immersion Capacitive Micromachined Ultrasonic Transducers on Silicon and Dielectric Substrates", IEEE Transactions on UFFC, In Press, Accepted May 2004

51. J.G. Knight and F.L. Degertekin, "Capacitive Micromachined Ultrasonic Transducers for Forward Looking Intravascular Imaging Arrays", Proceedings of 2002 IEEE Ultrasonics Symposium, pp. 1079-1082 (2002)

52. G.G. Yaralioglu, A.S. Ergun, B. Bayram, E. Hæggström, and B.T. Khuri-Yakub, "Calculation and Measurement of Electromechanical Coupling Coefficient of Capacitive Micromachined Ultrasonic Transducers", IEEE Trans. on UFFC, Vol. 50, No. 4, pp.449-456 (2003)

53. U. Schnakenberg, W. Benecke, and P. Lange, "TMAHW Etchants for Silicon Micromachining", Proceedings of 1991 International Conference on Transducers, pp.815-818 (1991)

54. F. Laermer and A. Schilp of Robert Bosch GmbH, "Method of Anisotropically Etching Silicon”, US-Patent No. 5501893 
55. F. Laermer, A. Schilp, K. Funk, and M. Offenberg, "Bosch Deep Silicon Etching: Improving Uniformity and Etch Rate for Advanced MEMS Applications", Proceedings of IEEE International Conference on MEMS, pp.211-216 (1999)

56. Unaxis 790 PECVD, Unaxis Semiconductors, St. Petersburg, FL 33716

57. J. Thurn, R.F. Cook, M. Kamorajugadda, and L. C. Stearns, "Stress Hysteresis and Mechanical Characterization of Plasma Enhanced Chemical Vapor Deposited Dielectrics", Mat. Res. Soc. Symp. Proc., Vol. 695, pp. L3.9.1-6 (2002)

58. T. S. Hickernell, F. M. Fliegel, and F. S. Hickernell, "The Elastick Properties of ThinFilm Silicon Nitride", Proceedings of 1990 IEEE Ultrasonics Symposium, pp 445-448 (1990)

59. J. Ashley Taylor, "The mechanical properties and microstructure of plasma enhanced chemical vapor deposited silicon nitride thin films", Journal of Vacuum Science \& Technology A: Vacuum, Surfaces, and Films, Vol. 9, Issue 4, pp. 2464-2468 (1991)

60. H. Baltes, O. Brand, A. Hierlemann, D. Lange, and C. Hagleitner, "CMOS MEMSPast, Present, and Future", Proceedings of IEEE International Conference on MEMS, pp.459-466 (2002)

61. S. D. Senturia, Microsystem Design, Kluwer Publishing, Boston, MA(2001)

62. F.V. Hunt, Electroacoustics, Acoustical Society of America, College Park, MD, pp.180-181 (1982)

63. I. Ladabaum, X. Jin, H.T. Soh, A. Atalar, and B.T. Khuri-Yakub, "Capacitive Micromachined Ultrasonic Transducers", IEEE Transactions on UFFC, Vol. 45, No.3, pp.678-690 (1998)

64. M. Badi, G.G. Yaralioglu, A. S. Ergun, S.T. Hansen, E.J. Wong, and B.T. KhuriYakub, "Capacitive Micromachined Ultrasonic Lamb Wave Transducers Using Rectangular Membranes", IEEE Transactions on UFFC, Vol. 50, No.9, pp.1191-1203 (2003)

65. C.E. Bradley, J.M. Bustillo, and R.M. White, "Flow Measurements in a Micromachined Flow System with Integrated Acoustic Pumping", Proceedings of 1995 IEEE Ultrasonics Symposium, pp.505-510 (1995)

66. H. Jagannathan, G.G. Yaralioglu, A.S. Ergun, F.L. Degertekin and B.T. KhuriYakub, "Micro-Fluidic Channels with Integrated Ultrasonic Transducers", Proceedings of 2001 IEEE Ultrasonics Symposium, pp.859-862 (2001)

67. Dow Website for Properties of Water-Glycerine mixtures, www.dow.com

68. A. D. Pierce, Acoustics: An Introduction to Its Physical Principles and Applications, Acoustical Society of America, Woodbury, New York, pp.30-34(1994)

69. NIST Thermophysical Properties of Pure Fluids Database, www.nist.gov, National Insitute of Standards and Technology, Gaithersburg, MD 
70. C.C. Leroy, "The Speed of Sound in Pure and Neptunian Water", in Handbook of Elastic Properties of Solids, Liquids, and Gases, Volume IV, Elastic Properties of Fluids: Liquids and Gases, Edited by M. Levy, D. Sinha, and R. Raspet, Academic Press, San Diego, pp.23-35 (2001)

71. D.T. Blackstock, Fundamentals of Physical Acoustics, Wiley \& Sons, New York, p.93 (2000)

72. L. Hall, "The Origin of Ultrasonic Absorption in Water", Physical Review, Vol. 73, No. 7, pp.775-781(1948)

73. S.M. Karim, and L. Rosenhead, "The Second Coefficient of Viscosity of Liquids and Gases", Reviews of Modern Physics, Vol. 24, No. 2, pp.108-116

74. J.M.M. Pinkerton, "The Absorption of Ultrasonic Waves in Liquids and its Relation to Molecular Constitution”, Proc. Phys. Soc. London, B62, pp.129-141(1947)

75. S.G. Joshi and Y. Jin, "Application of a Surface-Acoustic-Wave Device for Measurement of Liquid Flow Rate", IEEE Transactions on UFFC, Vol. 37, No.5, pp.475-477 (1990)

76. H. Eren, "Accuracy in Real Time Ultrasonic Applications and Transit Time Flow Meters", Proc. of IEEE Instrumentation and Measurement Technology Conference, pp.568-572 (1998)

77. J. Lighthill, Waves in Fluids, Cambridge University Press, Cambridge, pp.337-351 (1978)

78. W. Nyborg, "Acoustic Streaming", in Nonlinear Acoustics, edited by M.F. Hamilton and D.T. Blackstock, Academic Press, San Diego (1998)

79. W.L. Nyborg, "Sonically produced heat in a fluid with bulk viscosity and shear viscosity", J. Acoust. Soc. Am., Vol. 80, No. 4, pp.1133-1139 (1986)

80. www.kaptontape.com

81. G.T.A. Kovacs, Micromachined Transducers Sourcebook, McGraw-Hill, Boston (1998)

82. J. Jung and S.S. Lee, "Theoretical and experimental study of MHD (magnetohydrodynamic) micropump", Sensors and Actuators A, Vol. 80, pp.84-89 (2000)

83 P.F. van Kessel, L.J. Hornbeck, R.E. Meier, and M.R. Douglass, "A MEMS-Based Projection Display", Proceedings of the IEEE, Vol. 86, No. 8, pp.1687-1704 